

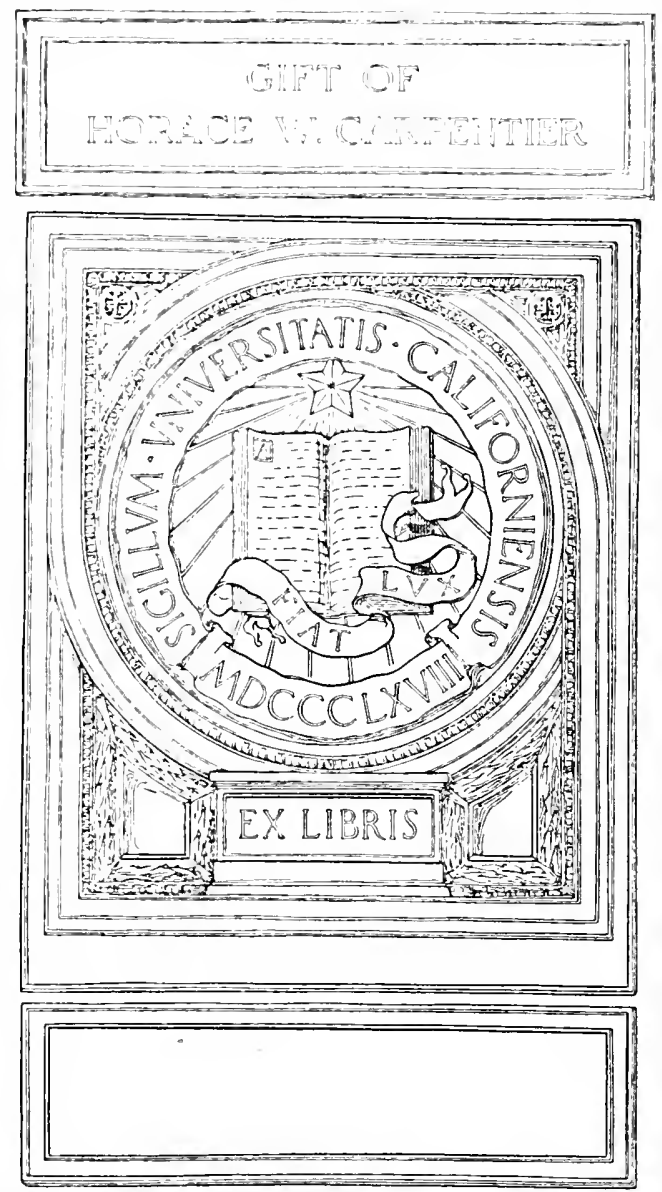


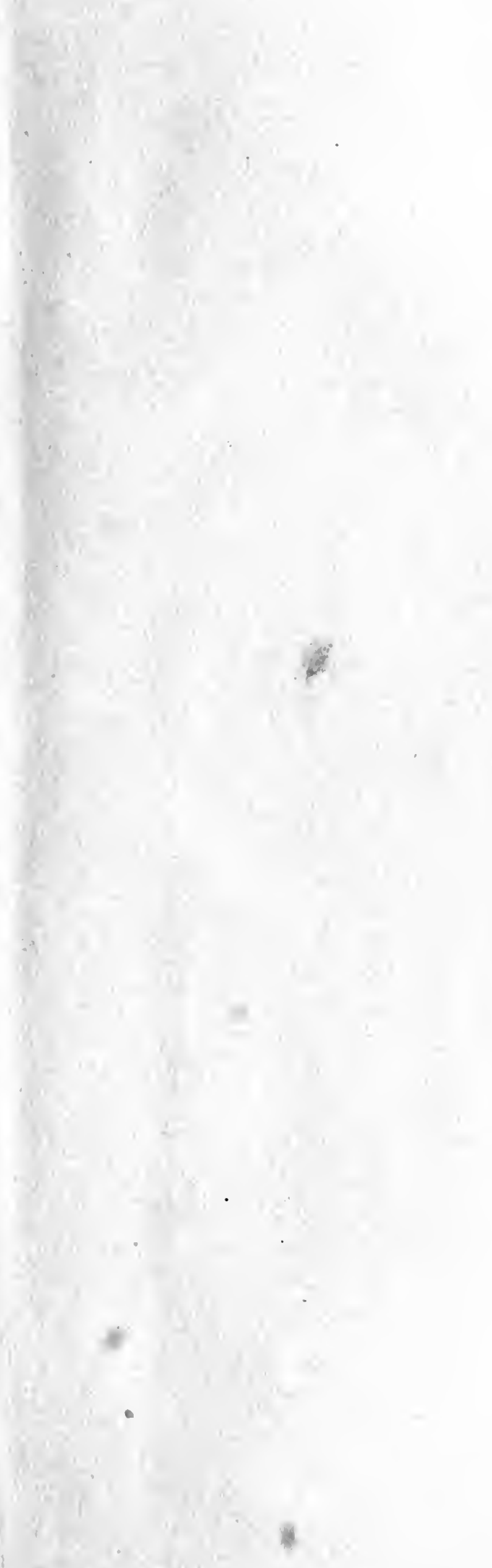


2 


\section{JUK 171932}

\section{STUDIA SINAITICA No. III.}

\section{CATALOGUE}

\section{OF THE ARABIC MSS.}

IN THE CONVENT OF S. CATHARINE ON

MOUNT SINAI

COMPILED BY

MARGARET DUNLOP GIBSON.

LONDON :

C. J. CLAY AND SONS,

CAMBRIDGE UNIVERSITY PRESS WAREHOUSE

AVE MARIA LANE.

I 894

Price Six Shillings, net. 

CATAlogue

OF ARABIC MSS. 
Zlondon: C. J. CLAY AND SONS, CAMBRIDGE UNIVERSITY IRESS WAREHOUSE, AVE MARIA LANE.

Gasgow: 263, ARGYLE STREET.

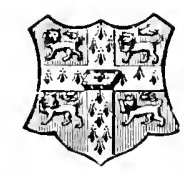

Uambrioge: DEIGHTON, BELL AND CO. Ilripzig: F. A, BROCKHAUS. Brow gork: NACHILLAN AND CO. 


$$
\text { - }
$$




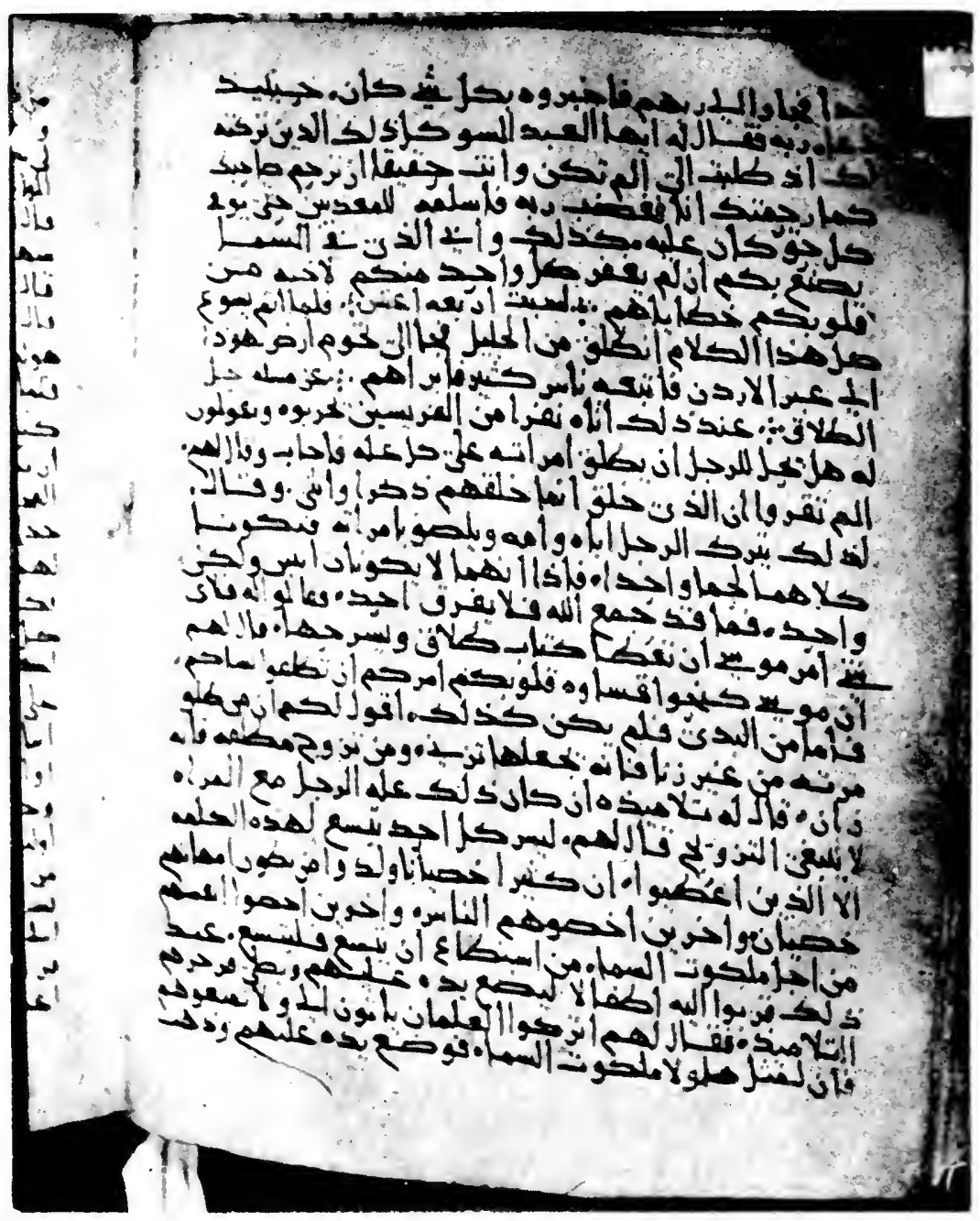




\section{STUDIA SINAITICA NO. III.}

\section{CATALOGUE}

\section{OF THE ARABIC MSS.}

IN THE CONVENT OF S. CATHARINE ON

MOUNT SINAI

COMPILED BY

MARGARET DUNLOP GIBSON.

LONDON :

C. J. CLAY AND SONS,

CAMBRIDGE UNIVERSITY PRESS WAREHOUSE

AVE MARIA LANE.

I 894

[All Rights reserved.] 


\section{vambrioge:}

PRINTED By C. J. Clay, M.A. AND SONS, AT THE UNIVERSITY PRESS. 


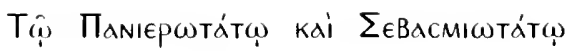

$$
\text { 'AрXıєпıско́тஸ }
$$

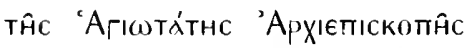

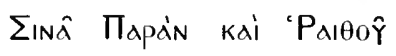

$$
\text { KYPI }
$$

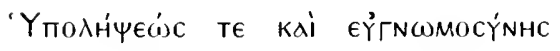

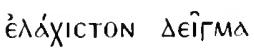

просфє́рєI

Hi CYNTÁKTHC 


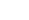




\section{INTRODUCTION.}

$\mathrm{N}^{\mathrm{N}}$ the IIth of January, I 893, when in Cairo with my sister, Mrs S. S. Lewis, preparing for our second visit to Mount Sinai, I asked and obtained permission from Archbishop Porphyrios to catalogue the Arabic and Syriac books in the library, which is equal or even superior to that at Jerusalem in the wealth of Christian Arabic MSS. it contains. His Beatitude was kind enough to write to the resident heads of the Convent, Fathers Galaktion and Nicodemus, desiring them to shew all the Arabic and Syriac books in their possession to Mrs Lewis and myself, that we might make catalogues of them, catalogues which we were to leave at the Convent. His letter is printed in the Introduction to No. I. of this series. As our stay was limited to forty days, it was impossible for me to do more than indicate the contents of each volume, without going into detail. There may therefore be some interesting things that have escaped my observation, but I trust that this list may be a guide to future travellers in making further researches. The volumes were all already numbered, and this had evidently been done with method, as may be seen by a glance at the plan of their arrangement. The monks carried out the orders of their Archbishop in the most cordial spirit, and without their active help I could not have performed a feat involving so much physical labour. 
Most of the books had lost not only their title-pages, but their last leaves as well, so that it was not possible to find their dates. One is ashamed to think that some scholar in former years must have abused the hospitality of the monks, and that a choice collection of title-pages may be found in some European library. There were a few, happily not many, whose fate it had been during the centuries of their existence not to be 'marked nor learned' but 'inwardly digested' by little rodents more innocent than scholars.

In conclusion, I have to express my thanks to $\mathrm{H}$ is Beatitude Porphyrios, Archbishop of Mount Sinai, and to the Fathers who gave me such active assistance; to Mrs Bensly, who shared with them the task of counting leaves; to $\mathrm{Mr}$ J. Rendel Harris, of Clare College, Lecturer in Palieography to the University of Cambridge, for giving me much valuable advice; to my dear sister, Mrs S. S. Lewis, who has revised my proofs, and who also helped me at the Convent by examining some fifty books, most of which I have marked thus + . I am likewise indebted to Mr A. Cowley, M.A. of Oxford, who has kindly furnished me with additional particulars of the volumes he examined during his visit to the Convent this year. These latter I have marked with an asterisk.

Margaret D. Gibson.

Castle-brak,

Canbridie.

$f_{117 y}, 1894$. 
ПIPOPAMATA.
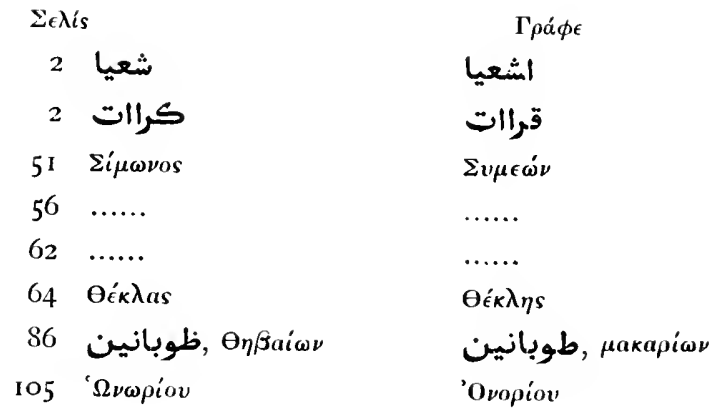

4

كتاب مقالات عن سيرة ابراهيم

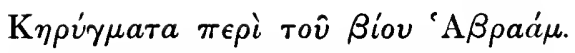

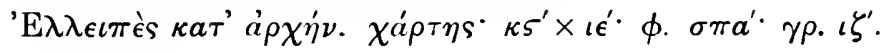

G. A. M. 
viii INTRODUCTION.

Most of the books had lost not only their title-pages, but their last leaves as well, so that it was not possible to 


\section{XЕIPOГРАФA АРАВIKA}

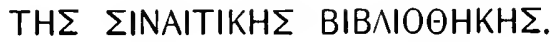

$$
\text { نبوة دانيال : نبوة ارميا : نبوة حزقيال }
$$

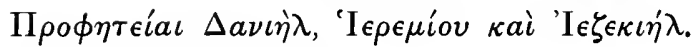

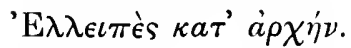

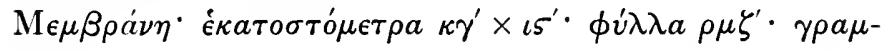
$\mu a \grave{\kappa} \kappa s^{\prime}$.

\section{2}

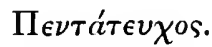

$$
\text { كتاب التوراة }
$$

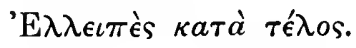

$\mathrm{X} a ́ \rho \tau \eta \varsigma^{\cdot} \kappa \varsigma^{\prime} \times \iota \epsilon^{\prime} \phi . \sigma \xi^{\prime} \cdot \gamma \rho . \iota \zeta^{\prime}$.

3

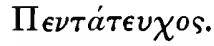

$$
\text { كتاب التوراة }
$$

$\mathrm{X}_{\alpha}^{\prime} \rho \tau \eta \varsigma^{\cdot} \kappa \delta^{\prime} \times \iota \zeta^{\prime} \cdot \phi . \tau o \gamma^{\prime} \cdot \gamma \rho . \iota 5^{\prime}$.

4

$$
\text { كتاب مقالات عن سيرة ابراهيم }
$$

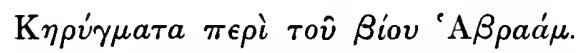

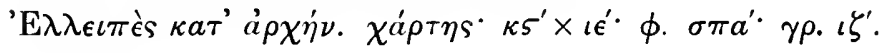

G. A. M. 
5

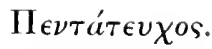

كتاب التوراة

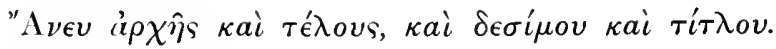

Х $а \dot{\rho} \tau \eta \varsigma^{\cdot} \kappa \beta^{\prime} \times \iota \epsilon^{\prime} \cdot \phi . \tau \zeta^{\prime} \cdot \gamma \rho . \iota \gamma^{\prime}$.

6

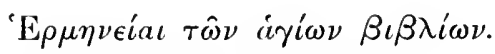

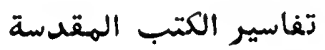

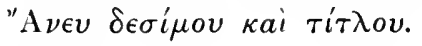

Х $\iota \rho \tau \eta \varsigma^{\cdot} \kappa a^{\prime} \times \iota \delta^{\prime} \cdot \phi . \rho \nu \theta^{\prime} \cdot \gamma \rho . \iota \epsilon^{\prime}$.

\section{7}

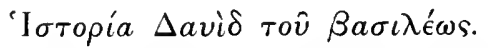

خبر داود الهالك

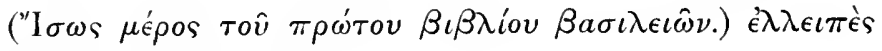

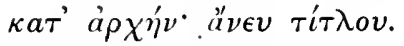

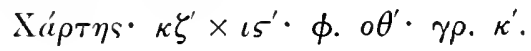

8

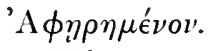

9

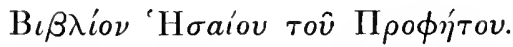

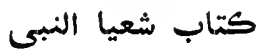

$\mathrm{X} a ́ \rho \tau \eta \varsigma^{*} \kappa \epsilon^{\prime} \times \iota \zeta^{\prime} \cdot \phi . \tau \xi \sigma^{\prime} \cdot \gamma \rho . \iota \zeta^{\prime} . \mathrm{M} \epsilon \tau a ̀ \mathrm{X} \rho \iota \sigma \tau o ̀ \nu, a \sigma \kappa \beta^{\prime}$.

10

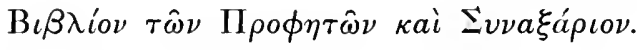

Хá $\rho \tau \eta \varsigma^{\cdot} \kappa \varsigma^{\prime} \times \iota \eta^{\prime} \cdot \phi . \sigma \epsilon^{\prime} \cdot \gamma \rho . \iota \theta^{\prime}$. M. X. $a \tau \eta^{\prime}$.

\section{1}

\section{كراات للاعياد من نبوة اشعيا}

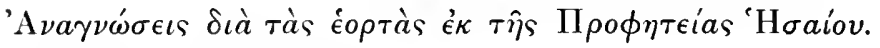

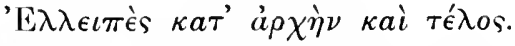

Х $а ́ \rho \tau \eta \varsigma^{\cdot} \iota \zeta^{\prime} \times \imath \beta^{\prime} \cdot \phi . \rho \nu \delta^{\prime} \cdot \gamma \rho . \imath \eta^{\prime}$. 
12

$$
\text { كتاب نبوات : قرالت للاعياد }
$$

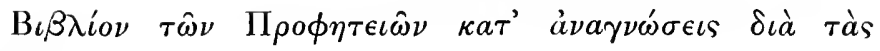
éoptás.

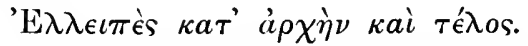

$\mathrm{X} \alpha \dot{\rho} \tau \eta \varsigma^{*} \kappa a^{\prime} \times \iota \epsilon^{\prime} \cdot \phi . \rho \lambda \gamma^{\prime} \cdot \gamma \rho . \iota \delta^{\prime}$.

\section{3}

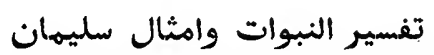

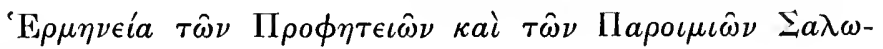
$\mu \hat{\omega} \nu \tau o s$.

$\mathrm{X} \alpha \dot{\rho} \rho \eta \varsigma^{*} \kappa \epsilon^{\prime} \times \iota \varsigma^{\prime} \cdot \phi . \tau \pi \gamma^{\prime} \gamma \rho . \iota \zeta^{\prime}$. M. X. , $a \sigma \kappa \beta^{\prime}$.

\section{4}

$\Pi \rho \circ \phi \eta т о \lambda o ́ \gamma \iota о \nu$.

$$
\text { قرالت من الانبيا }
$$

$\mathrm{X} a ́ \rho \tau \eta \varsigma^{\cdot} \lambda^{\prime} \times \kappa^{\prime} \cdot \phi . \rho \tau \beta^{\prime} \cdot \gamma \rho . \kappa \gamma^{\prime}$.

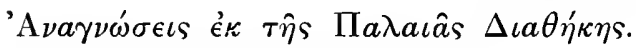

قراات من العهد القديم

$\mathrm{X} \alpha \dot{\rho} \rho \tau \varsigma^{*} \kappa \gamma^{\prime} \times \iota \epsilon^{\prime} \cdot \phi . \rho \mu \varsigma^{\prime} \cdot \gamma \rho . \kappa a^{\prime}$.

\section{6}

$$
\text { قرات رلاعياد من الهزامير }
$$

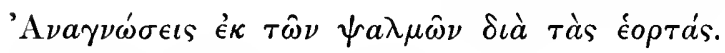

$\mathrm{X} \alpha^{\prime} \rho \tau \eta \varsigma^{\prime} \iota \eta^{\prime} \times \iota \gamma^{\prime} \cdot \phi . \sigma \kappa \epsilon^{\prime} \cdot \gamma \rho . \iota \gamma^{\prime}$.

\section{7}

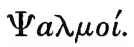

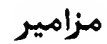

$\mathrm{X} a ́ \rho \tau \eta \varsigma^{\cdot} \iota \delta^{\prime} \times \iota a^{\prime} \phi . \rho \pi a^{\prime} \cdot \gamma \rho . \iota \beta^{\prime}$.

\section{8}

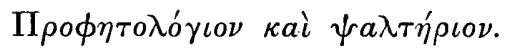

$$
\text { قرالت من الانبيا والهزامير }
$$

$\mathrm{X} a ́ \rho \tau \eta \varsigma^{\cdot} \kappa^{\prime} \times \iota \delta^{\prime} \cdot \phi . \sigma \kappa \theta^{\prime} \cdot \gamma \rho .{ }^{\prime} \delta^{\prime}$. 
'A $\phi \eta \rho \eta \mu \epsilon^{\prime} \nu o \nu$.

\section{0}

$\Psi a \lambda \tau$ Típเov.

$$
\text { كتّاب المزامير }
$$

M $\epsilon \mu \beta \rho c^{\prime} \nu \eta^{\cdot} \iota 5^{\prime} \times \iota a^{\prime} \cdot \phi . \sigma \kappa \theta^{\prime} \cdot \gamma \rho . \theta^{\prime}$.

\section{1}

$\Psi a \lambda \tau i ́ p$

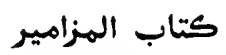

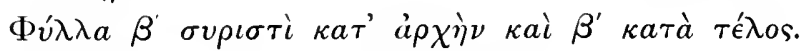

$\mathrm{M} \epsilon \mu \beta \rho a^{\prime} \nu \eta^{\cdot} \iota \delta^{\prime} \times \iota^{\prime} \phi . \rho \uparrow \beta^{\prime} \cdot \gamma \rho . \iota \beta^{\prime}$.

\section{2}

$\Psi a \lambda \tau i ́ \rho \rho o \nu$.

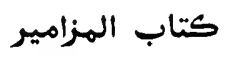

'E $\mathrm{\lambda} \lambda \epsilon \iota \pi \dot{\epsilon} \varsigma \kappa a \tau$ ' $\dot{a} \rho \chi \eta \dot{\eta} \nu$.

$\mathrm{M} \epsilon \mu \beta \rho a^{\prime} \nu \eta \cdot \iota \epsilon^{\prime} \times \iota a^{\prime} \cdot \phi . \sigma o a^{\prime} \cdot \gamma \rho . \iota \eta^{\prime}$.

23

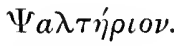

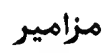

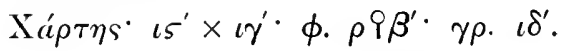

\section{4}

$\Psi a \lambda \tau i ́ p\llcorner o v$.

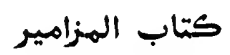

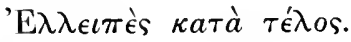

$\mathrm{X} a ́ \rho \tau \eta \varsigma^{\prime} \iota \epsilon^{\prime} \times \iota^{\prime} \phi . \sigma \iota \beta^{\prime} \cdot \gamma \rho . \iota \beta^{\prime}$.

\section{5}

$\Psi a \lambda \tau i ́ \rho\llcorner o \nu$.

كتاب الهزامير

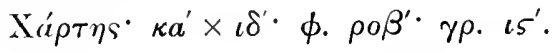

26

$\Psi a \lambda \tau \eta \dot{\rho} \rho \circ$.

$$
\text { كتاب الهزامير }
$$

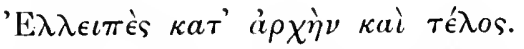

$\mathrm{X} \operatorname{la}^{\rho} \rho \eta \varsigma^{\cdot} \iota \eta^{\prime} \times \iota \gamma^{\prime} \phi . \rho \mu \beta^{\prime} \cdot \hat{\omega} \nu \zeta^{\prime} \delta \iota a \sigma \pi a \sigma \mu \epsilon^{\prime} \nu a \cdot \gamma \rho . \iota \gamma^{\prime}$. 


\section{7}

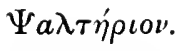

$$
\text { كتاب المزامير }
$$

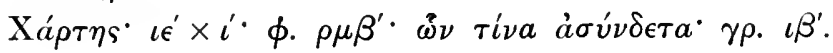

28

$\Psi a \lambda \tau \eta \dot{\rho} \rho\llcorner$.

$$
\text { كتاب الهزامير }
$$

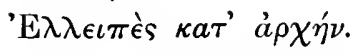

$\mathrm{X} a ́ \rho \tau \eta \varsigma^{\cdot} \iota \zeta^{\prime} \times \iota \beta^{\prime} \cdot \phi . \sigma \iota \theta^{\prime} \cdot \gamma \rho . \iota \beta^{\prime}$. M. X. $a \sigma \xi \theta^{\prime}$.

\section{9}

$\Psi a \lambda \tau \eta \dot{\rho} \iota \mathrm{\nu}$.

$$
\text { كتاب الهزامير }
$$

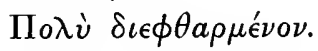

$\mathrm{X} a ́ \rho \tau \eta s^{\cdot} \iota \delta^{\prime} \times \iota^{\prime} \cdot \phi . \rho \iota^{\prime} \gamma \rho . \imath \beta^{\prime}$.

30

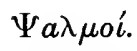

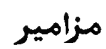

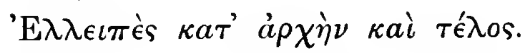

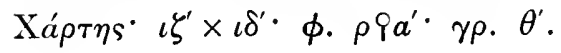

\section{1}

$\Psi a \lambda \tau \eta \dot{\rho} \iota \circ \nu$.

مزامير

$\mathrm{X} a \dot{\rho} \tau \eta \varsigma^{\cdot} \kappa \beta^{\prime} \times \iota \epsilon^{\prime} \cdot \phi .9 a^{\prime} \gamma \rho . \iota \zeta^{\prime}$.

32

$\Psi a \lambda \tau \eta \dot{p} \iota \mathbf{\nu}$.

مزامير

'E $\lambda \lambda \epsilon \iota \pi \grave{\epsilon} \varsigma \kappa a \tau$ ' à $\rho \chi \eta \dot{~} \nu$.

$\mathrm{X} a ́ \rho \tau \eta \varsigma^{\cdot} \iota \epsilon^{\prime} \times \iota a^{\prime} \phi, \rho \lambda \zeta^{\prime} \gamma \rho . \iota^{\prime}$.

\section{3}

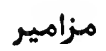

$\Psi a \lambda \tau \eta \dot{\rho} \iota \nu$.

$\mathrm{X} a ́ \rho \tau \eta \varsigma^{\cdot} \iota \theta^{\prime} \times \iota \eta^{\prime} \phi . \rho \nu \beta^{\prime} \gamma \rho . \iota \beta^{\prime}$. 


\section{4}

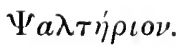

$$
\text { كتاب المزامير }
$$

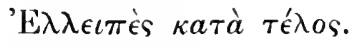

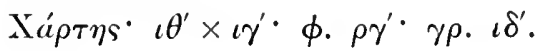

\section{5}

$\Psi a \lambda \tau \eta^{\prime} \rho \iota \nu$.

$$
\text { كتاب الهزامير }
$$

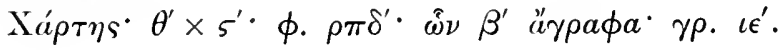

\section{6}

$\Psi a \lambda \tau i \rho \rho \circ$.

مزامير

$\mathrm{X} a ́ \rho \tau \eta \varsigma^{*} \iota \epsilon^{\prime} \times \iota a^{\prime} \cdot \phi, \rho \nu \beta^{\prime} \cdot \gamma \rho, \iota a^{\prime}$.

\section{7}

$\Psi a \lambda \tau \eta \dot{p} \rho \circ$.

كتاب الهزامير

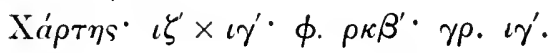

\section{8}

$\Psi a \lambda \tau \dot{\eta} \rho\llcorner\circ \nu$.

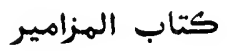

$\mathrm{X} a ́ \rho \tau \eta \varsigma^{\cdot} \iota \zeta^{\prime} \times \iota \gamma^{\prime} \cdot \phi . \rho \mu \eta^{\prime} \cdot \gamma \rho . \iota \delta^{\prime}$.

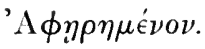

\section{9}

40

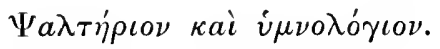

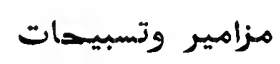

$\mathrm{X} a ́ \rho \tau \eta \varsigma^{\cdot} \iota \zeta^{\prime} \times \iota \beta^{\prime} \cdot \phi . \tau \lambda^{\prime} \cdot \gamma \rho . \iota \delta^{\prime}$.

\section{1}

$\Psi a \lambda \tau r^{\prime} \rho\llcorner o \nu$.

كتاب الهزامير

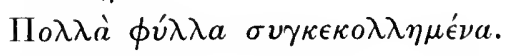

$\mathrm{X} \alpha \dot{\alpha} \tau \eta \varsigma^{\cdot} \iota \delta^{\prime} \times \iota^{\prime} \cdot \phi . \rho o \zeta^{\prime} \cdot \gamma \rho . \iota a^{\prime}$. 


\section{2}

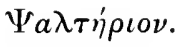

$$
\text { كتاب الهزامير }
$$

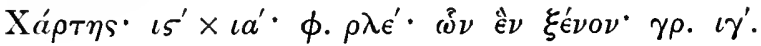

43

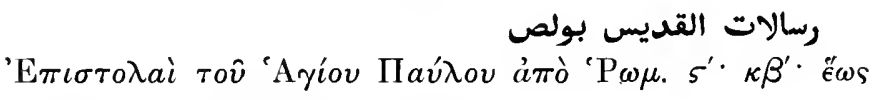
Т $\iota \mu . \mathrm{B}^{\prime} \cdot \gamma^{\prime} \eta^{\prime} . \quad \mathrm{M} \epsilon \mu \beta \rho a ́ \nu \eta^{*} \iota \epsilon^{\prime} \times \iota \beta^{\prime} \cdot \phi .95^{\prime} \gamma \rho . \iota \eta^{\prime}$.

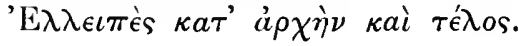

43 bis

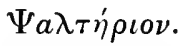

كتاب المزامير

$\mathrm{X} \alpha \rho \rho \eta s^{*} \iota \eta^{\prime} \times \iota \gamma^{\prime} \phi . \rho o \beta^{\prime} \gamma \rho . \iota \gamma^{\prime}$.

\section{4}

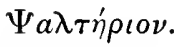

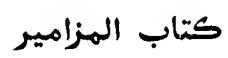

$\mathrm{X} a \rho \tau \eta \varsigma^{\cdot} \kappa^{\prime} \times \iota \delta^{\prime} \cdot \phi . \rho \nu \delta^{\prime} \gamma \rho . \iota \epsilon^{\prime}$.

\section{5}

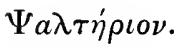

كتاب الهزامير

$\mathrm{X} \alpha \dot{\rho} \rho \eta s^{*} \iota \zeta^{\prime} \times \iota \gamma^{\prime} \phi . \rho \iota \eta^{\prime} \gamma \rho . \iota \gamma^{\prime}$.

\section{6}

$\Psi a \lambda \tau \eta \dot{\rho} \iota$ เ

كتاب الهزامير

$\mathrm{X} a \dot{\rho} \tau \tau \eta \varsigma^{\cdot} \iota \epsilon^{\prime} \times \iota \beta^{\prime} \cdot \phi, \sigma \kappa^{\prime} \gamma \rho, \iota \beta^{\prime}$.

\section{7}

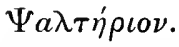

$$
\text { كتاب المززامير }
$$

$\mathrm{X} a \rho \rho \eta s^{*} \kappa a^{\prime} \times \iota \delta^{\prime} \phi . \rho \eta^{\prime} \gamma \rho . \iota \gamma^{\prime}$.

\section{8}

$\Psi a \lambda \tau \eta \dot{\rho} \iota \nu \nu$.

$$
\text { كتاب الهزامير }
$$

$\mathrm{X} a ́ \rho \tau \eta \varsigma^{\cdot} \iota \theta^{\prime} \times \iota \gamma^{\prime} \phi . \sigma \iota^{\prime} \gamma \rho . \iota \beta^{\prime}$. 
$\Psi a \lambda \tau \dot{p} \rho\llcorner o \nu$.

كتاب الهزامير

$\mathrm{X} \alpha \rho \tau \eta \varsigma^{\cdot} \iota \gamma^{\prime} \times \iota \zeta^{\prime} \phi . \sigma \iota \varsigma^{\prime} \gamma \rho . \iota a^{\prime}$.

50

$\Psi a \lambda \tau \eta_{\rho}\llcorner\circ \nu$.

$$
\text { كتاب الهزامير }
$$

$\mathrm{X}^{\prime} \rho \tau \eta \varsigma^{\cdot} \iota 5^{\prime} \times \iota \beta^{\prime} \cdot \phi . \sigma \lambda \epsilon^{\prime} \gamma \rho . \iota \delta^{\prime}$.

51

$\Psi a \lambda \tau \eta^{\prime} \rho \iota \nu \nu \mu \in \tau \grave{a} \tau \hat{\omega} \nu \theta^{\prime} \dot{\omega} \delta \hat{\omega} \nu$.

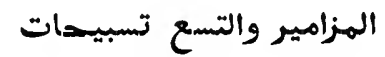

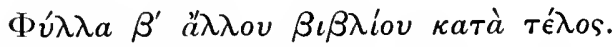

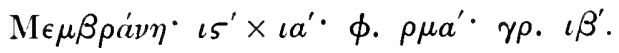

\section{2}

$\Psi a \lambda \tau \operatorname{ro}^{\prime} \rho \mathrm{L}$.

كتاب الهزامير

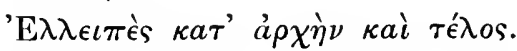

$\mathrm{X} a ́ \rho \tau \eta \varsigma^{\cdot} \iota \delta^{\prime} \times \iota^{\prime}{ }^{\prime} \phi . \sigma \lambda \zeta^{\prime} \gamma \rho . \iota a^{\prime}$.

\section{3}

$\Psi a \lambda \tau \eta \dot{p} \rho \circ \nu$.

كتاب الهزامير

$\mathrm{X} a ́ \rho \tau \eta \varsigma^{*} \kappa \varsigma^{\prime} \times \iota \zeta^{\prime} \cdot \phi \cdot \rho \nu \epsilon^{\prime} \cdot \gamma \rho . \iota \delta^{\prime}$.

54

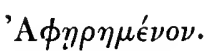

\section{5}

$\Psi a \lambda \tau \dot{\eta} \rho\llcorner о \nu$.

كتاب الهزامير

$\Pi o \lambda \dot{v} \delta \iota \epsilon \phi \theta a \rho \mu \epsilon^{\prime} \nu o \nu$.

$\mathrm{X} \alpha \dot{\alpha} \rho \eta \varsigma^{\cdot} \iota 5^{\prime} \times \iota \gamma^{\prime} \phi . \sigma \lambda a^{\prime} \cdot \gamma \rho . \iota \beta^{\prime}$. 
56

$\Psi a \lambda \tau \eta \dot{\rho} \iota \circ \nu$.

كتاب الهززامير

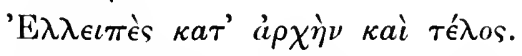

$\mathrm{X} \dot{a} \rho \tau \eta \varsigma^{\cdot} \iota \delta^{\prime} \times \iota^{\prime} \phi . \rho \mu \eta^{\prime} \cdot \gamma \rho \cdot \iota a^{\prime}$.

57

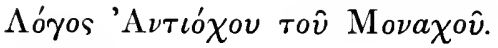

من قول انتخس الراهب : مزامير

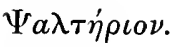

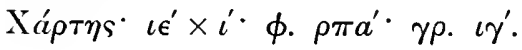

58

$\Psi a \lambda \tau \dot{\eta} \rho \iota \nu \nu$

مزامير الهقدسة

$\mathrm{X} a^{\prime} \rho \tau \eta \varsigma^{*} \iota \zeta^{\prime} \times \iota a^{\prime} \cdot \phi . \rho \lambda \zeta^{\prime} \gamma \rho . \iota \gamma^{\prime}$.

59

$\Psi a \lambda \tau \dot{\eta} \rho \iota 0 \nu$.

كتاب الهزامير

$\mathrm{X} a ́ \rho \tau \eta s^{\cdot} \iota \delta^{\prime} \times \iota^{\prime} \phi . \rho \xi \beta^{\prime} \cdot \gamma \rho . \iota \gamma^{\prime}$.

$\Psi a \lambda \tau \eta ́ p \iota \% \nu$

60

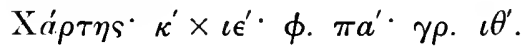

\section{1}

$\Psi a \lambda \tau \dot{\eta} \rho \iota 0 \nu$.

كتاب الهزامير

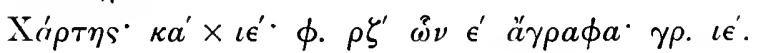

\section{2}

$\Psi a \lambda \tau \dot{\eta} \rho\llcorner o \nu$.

كتاب الهزامير

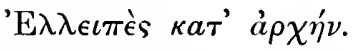

$\mathrm{X} a ́ \rho \tau \eta \varsigma^{\prime} \iota \gamma^{\prime} \times \theta^{\prime} \cdot \phi . \rho \xi \delta^{\prime} \cdot \gamma \rho . \theta^{\prime}$.

G. A. M. 
63

$\Psi a \lambda \tau \dot{\eta} \rho \iota \nu$.

كتاب الهزامير

$\mathrm{X} \alpha \rho \tau \eta \varsigma^{\cdot} \iota \epsilon^{\prime} \times \iota \beta^{\prime} \cdot \phi . \tau \lambda \gamma^{\prime} \gamma \rho . \iota a^{\prime}$.

\section{4}

'А

\section{5}

$\Psi a \lambda_{\text {T }} \dot{\rho} \iota \mathrm{\nu}$.

$$
\text { كتاب الهزامير }
$$

Х $\alpha^{\prime} \rho \tau \eta \varsigma^{\cdot} \iota \zeta^{\prime} \times \iota \gamma^{\prime} \cdot \phi . \quad \sigma \nu \delta^{\prime} \cdot \gamma \rho . \iota a^{\prime}$.

\section{6}

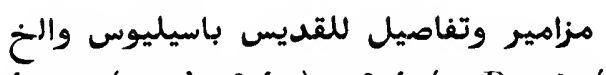

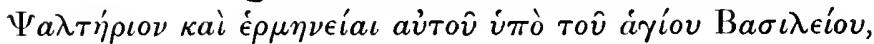
$\kappa . \tau . \lambda$.

Х $\dot{\alpha} \rho \tau \eta \varsigma^{\cdot} \kappa^{\prime} \times \iota \delta^{\prime} \cdot \phi \cdot \tau \uparrow \varsigma^{\prime} \cdot \gamma \rho . \iota \gamma^{\prime}$.

\section{7}

$\Psi a \lambda \tau \eta_{\rho} \iota \mathrm{\nu}$.

كتاب الهزامير

$\mathrm{X} a ́ \rho \tau \eta \varsigma^{\cdot} \kappa \beta^{\prime} \times \iota \delta^{\prime} \cdot \phi . \sigma \iota \gamma^{\prime} \cdot \gamma \rho . \iota \epsilon^{\prime}$.

68

'А $\phi \eta \rho \eta \mu{ }^{\prime} \nu 0 \nu$.

\section{9}

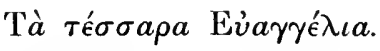

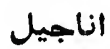

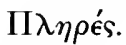

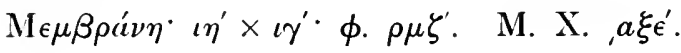

70

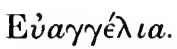

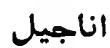

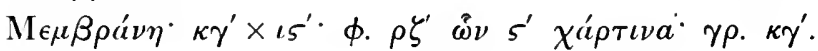




\section{1}

Ev่aryétıı.

اناجيل

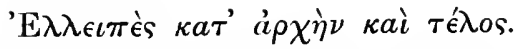

М $\epsilon \mu \beta \rho a ́ \nu \eta \cdot \kappa \beta^{\prime} \times \iota \zeta^{\prime} \cdot \phi . \mu \eta^{\prime} \cdot \gamma \rho . \iota \epsilon^{\prime}$.

\section{2}

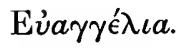

اناجيل

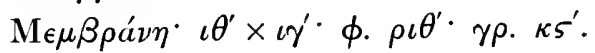

\section{3}

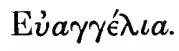

اناجيل

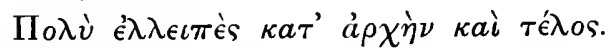

$\mathrm{M} \epsilon \mu \beta \rho a ́ \nu \eta \cdot \kappa a^{\prime} \times \iota 5^{\prime} \cdot \phi .9 \epsilon^{\prime} \cdot \gamma \rho . \kappa a^{\prime}$.

\section{4}

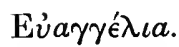

\section{اناجيل}

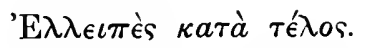

$\mathrm{M} \epsilon \mu \beta \rho a ́ \nu \eta \cdot \iota \varsigma^{\prime} \times \iota^{\prime} \phi . \sigma \nu^{\prime} \cdot \gamma \rho . \iota \delta^{\prime} \cdot a i \hat{\omega} \nu o s \quad \iota^{\prime}$.

\section{5}

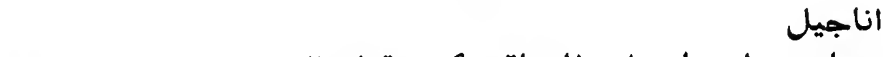

برهان يدل على ان للخلق يكسر قول الدهال الدهرين الذين يزعموا

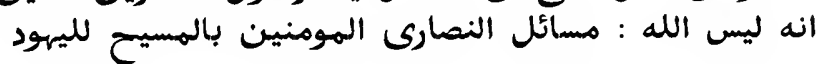

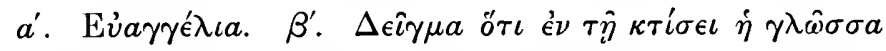

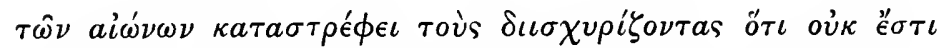

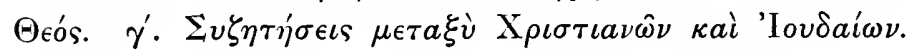
$\mathrm{M} \epsilon \mu \beta \rho a ́ \nu \eta \cdot \kappa \gamma^{\prime} \times \iota 5^{\prime} \cdot \phi . \sigma \iota \theta^{\prime} \cdot \gamma \rho . \kappa \eta^{\prime} \cdot \operatorname{ai} \hat{\omega} \nu o s \iota^{\prime}$.

\section{6}

Ev่aryé $\operatorname{\lambda } \iota a$.

$$
\text { اناجيل }
$$

$\mathrm{M} \epsilon \mu \beta \rho a ́ \nu \eta \cdot \kappa \zeta^{\prime} \times \iota \theta^{\prime} \cdot \phi . \tau \kappa^{\prime} \cdot \gamma \rho . \iota \gamma^{\prime}$. 
Ev̀aryédıa.

اناجيل

$\mathrm{X} a ́ \rho \tau \eta \varsigma^{*} \kappa^{\prime} \times \iota \delta^{\prime} \cdot \phi . \sigma \lambda \epsilon^{\prime} \cdot \gamma \rho . \iota \gamma^{\prime}$.

78

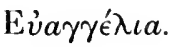

اناجيل

$\mathrm{X} a \rho \tau \eta \varsigma^{\cdot} \kappa \beta^{\prime} \times \iota \delta^{\prime} \cdot \phi . \tau \iota \gamma^{\prime} \gamma \rho . \iota \delta^{\prime}$. M. X. $a \sigma \pi \zeta^{\prime}$.

\section{9}

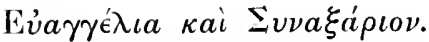

$\mathrm{X} a ́ \rho \tau \eta \varsigma^{\cdot} \kappa \beta^{\prime} \times \iota \gamma^{\prime} \phi . \tau \iota^{\prime} \gamma \rho . \iota \epsilon^{\prime}$. M. X. $a \tau \iota \theta^{\prime}$.

\section{0}

Ev̉a $a \gamma \hat{\epsilon} \lambda \iota a$.

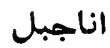

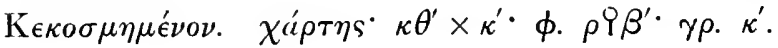

\section{1}

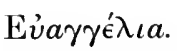

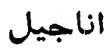

Хá $\rho \tau \eta \varsigma^{\cdot} \kappa \zeta^{\prime} \times \iota \eta^{\prime} \phi \phi . \rho \pi^{\prime} \cdot \gamma \rho . \iota \theta^{\prime}$. M. X. $a \sigma \nu a^{\prime}$.

\section{2}

Ė̉a

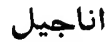

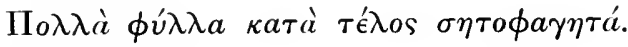

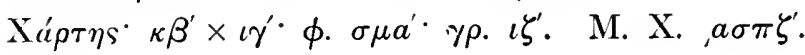

\section{3}

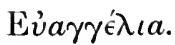

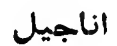

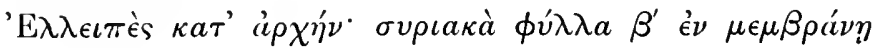
$\dot{\epsilon} \nu \tau \hat{\varphi} \delta \epsilon \sigma i \mu \omega$.

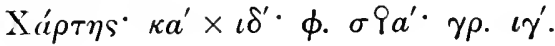


84

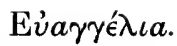

$$
\text { اناجيل }
$$

$\mathrm{X} a ́ \rho \tau \eta \varsigma^{\cdot} \kappa^{\prime} \times \iota \delta^{\prime} \cdot \phi . \sigma \mu \delta^{\prime} \stackrel{\oplus}{\omega} \epsilon^{\prime} \dot{\epsilon} \lambda \lambda \eta \nu \iota \kappa a^{\prime} \gamma \rho . \iota \zeta^{\prime}$.

\section{5}

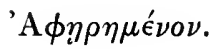

86

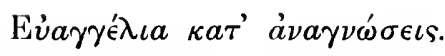

اناجيل

$\mathrm{X} a ́ \rho \tau \eta \varsigma^{\prime} \kappa a^{\prime} \times \iota \gamma^{\prime} \cdot \phi . \rho 9 \gamma^{\prime} \cdot \gamma \rho . \kappa^{\prime}$.

\section{7}

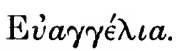

اناجيل

$\mathrm{X}^{\prime} \rho \tau \eta s^{\cdot} \kappa a^{\prime} \times \iota \epsilon^{\prime} \cdot \phi \cdot \rho \pi \beta^{\prime} \cdot \gamma \rho . \iota \eta^{\prime}$.

\section{8}

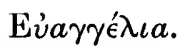

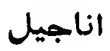

$\mathrm{X} \dot{\rho} \rho \tau \eta \varsigma^{\cdot} \kappa a^{\prime} \times \iota \delta^{\prime} \cdot \phi . \tau \zeta^{\prime} \cdot \gamma \rho . \iota \epsilon^{\prime}$.

89

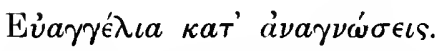

$\left.\mathrm{X} a ́ \rho \tau \eta \varsigma^{\cdot} \kappa \eta^{\prime} \times \kappa a^{\prime} \cdot \phi . \rho\right\} \delta^{\prime} \cdot \gamma \rho . \iota \eta^{\prime}$. M. X. $a \sigma \pi \zeta^{\prime}$.

\section{0}

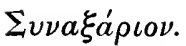

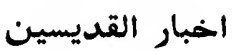

$\mathrm{X} a^{\prime} \rho \tau \eta \varsigma^{\cdot} \kappa \beta^{\prime} \times \iota \delta^{\prime} \cdot \phi . \tau a^{\prime} \cdot \gamma \rho . \iota \delta^{\prime}$.

91

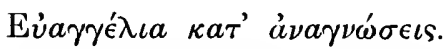

$\mathrm{X} a^{\prime} \rho \tau \eta \varsigma^{\cdot} \kappa \beta^{\prime} \times \iota \delta^{\prime} \cdot \phi . \sigma \mu \theta^{\prime} \cdot \gamma \rho . \iota \varsigma^{\prime}$. M. X.,$a \sigma \pi \theta^{\prime}$. 


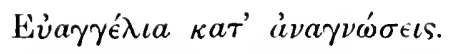

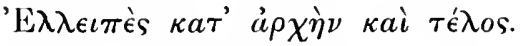

Х $а \dot{\rho} \tau \eta \varsigma^{\cdot} \iota \eta^{\prime} \times \iota \beta^{\prime} \cdot \phi$. $\rho \epsilon^{\prime} \cdot \gamma \rho$. $\iota \epsilon^{\prime}$.

93

Eن̀aryétıı.

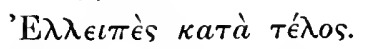

$\mathrm{X} a \dot{\rho} \tau \tau \eta \varsigma^{*} \kappa \theta^{\prime} \times \kappa^{\prime} \cdot \phi . \sigma \kappa \varsigma^{\prime} \cdot \gamma \rho . \quad \iota \epsilon^{\prime}$.

\section{4}

Ev̀a

$$
\text { اناجيل }
$$

Хá $\rho \tau \eta \varsigma^{*} \iota \theta^{\prime} \times \imath \beta^{\prime} \cdot \phi . \sigma \xi^{\prime} \cdot \gamma \rho . \iota 5^{\prime}$.

\section{5}

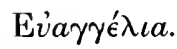

\section{اناجيل}

Х $a \rho \tau \eta \varsigma^{*} \iota \eta^{\prime} \times \iota \delta^{\prime} \cdot \phi . \tau \kappa \theta^{\prime} \cdot \gamma \rho . \iota \delta^{\prime}$. M. X.,$a \sigma o \beta^{\prime}$.

96

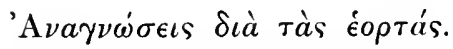

قراוت للاعياد

Ха́ $\rho \tau \eta \varsigma^{\prime} \kappa \gamma^{\prime} \times \iota \epsilon^{\prime} \cdot \phi . \tau \lambda \delta^{\prime} \cdot \gamma \rho . \iota \varsigma^{\prime}$.

97

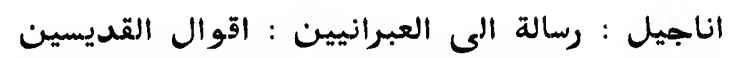

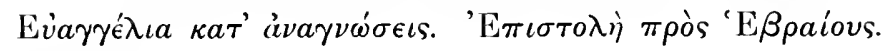

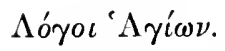

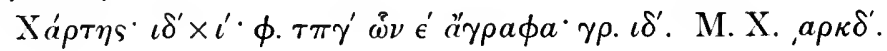

\section{8}

$$
\text { اناجيل وقراات من رسائل القديس بولص }
$$

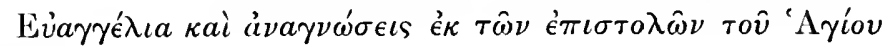
Паи́хov.

$\mathrm{X} a ́ \rho \tau \eta s^{\cdot} \iota \theta^{\prime} \times \iota \gamma^{\prime} \cdot \phi . \sigma a^{\prime} \cdot \gamma \rho . \iota \eta^{\prime}$. 
99

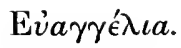

اناجيل

Х'㇒⿻

100

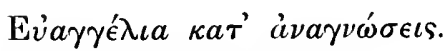

اناجيل

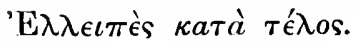

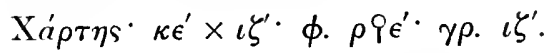

\section{1}

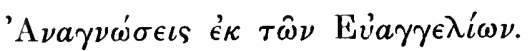

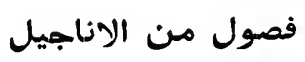

$\mathrm{X} a \dot{\rho} \tau \eta \varsigma^{\cdot} \iota \gamma^{\prime} \times \eta^{\prime} \cdot \phi . \tau \eta^{\prime} \cdot \gamma \rho . \iota^{\prime}$.

102

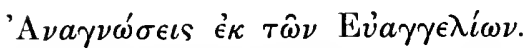

$$
\text { قراات من الاناجيل }
$$

$\mathrm{X} \alpha^{\prime} \rho \tau \eta \varsigma^{\cdot} \kappa^{\prime} \times \iota \gamma^{\prime} \phi . \sigma \eta^{\prime} \gamma \rho . \iota \epsilon^{\prime}$.

103

$\sum v \nu a \xi a ́ p \iota 0 \nu$.

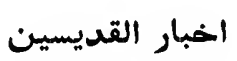

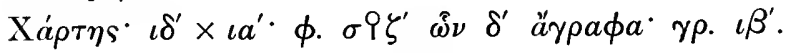

\section{4}

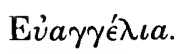

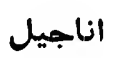

$\mathrm{X}^{\prime} \rho \tau \eta \varsigma^{\cdot} \quad \kappa a^{\prime} \times \iota \delta^{\prime} \cdot \phi . \quad \sigma \xi \theta^{\prime} \quad \hat{\omega} \nu \gamma^{\prime} \quad a^{\prime} \gamma \rho a \phi a^{\cdot} \gamma \rho . \quad \iota \epsilon^{\prime}$.

M. X. $a \sigma \pi a^{\prime}$.

\section{5}

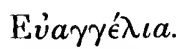

$$
\text { اناجيل }
$$

$\mathrm{X} a ́ \rho \tau \eta \varsigma^{\cdot} \kappa^{\prime} \times \iota \gamma^{\prime} \phi . \quad \tau \pi^{\prime} \cdot \gamma \rho . \iota \epsilon^{\prime} . \quad$ M. X. $a \tau \iota \beta^{\prime}$.

\section{6}

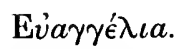

\section{اناجميل}

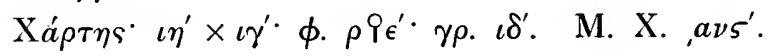




\section{7}

'E $\rho \mu \eta \nu \in i a \iota \tau \hat{\omega} \nu$ E $\dot{v} a \gamma \gamma \epsilon \lambda i \omega \nu$.

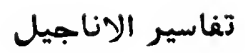

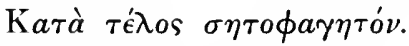

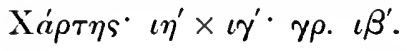

108

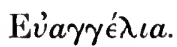

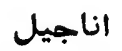

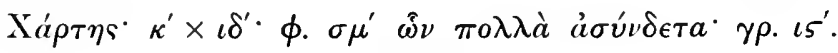

109

Ev̉a

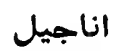

$\mathrm{X} \alpha^{\prime} \rho \tau \eta \varsigma^{*} \kappa^{\prime} \times \iota \delta^{\prime} \cdot \phi . \sigma \pi \sigma^{\prime} \cdot \gamma \rho . \iota \delta^{\prime}$. M. X.,$a \sigma \pi \zeta^{\prime}$.

110

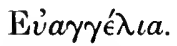

اناجيل

$\mathrm{X} a \dot{\rho} \tau \eta \varsigma^{\cdot} \kappa \beta^{\prime} \times \iota \delta^{\prime} \cdot \phi . \sigma \lambda \gamma^{\prime} \gamma \rho . \iota \zeta^{\prime}$.

\section{1}

Eن̉aryé $\lambda \iota a$.

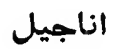

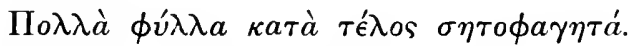

$\mathrm{X} \alpha^{\prime} \rho \tau \eta \varsigma^{*} \iota \eta^{\prime} \times \iota \gamma^{\prime} \phi$. $v \iota \gamma^{\prime} \cdot \gamma \rho . \iota \beta^{\prime}$.

\section{2}

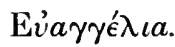

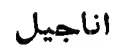

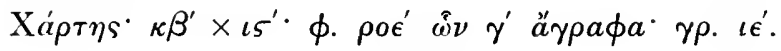

\section{3}

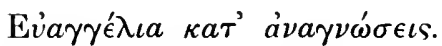

اناجيل

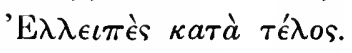

$\mathrm{X} a ́ \rho \tau \eta \varsigma^{\cdot} \kappa \epsilon^{\prime} \times \iota \zeta^{\prime} \cdot \phi \cdot \rho \pi a^{\prime} \cdot \gamma \rho . \iota \zeta^{\prime}$.

114

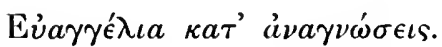

اناجيل

$\mathrm{X} \dot{a} \rho \tau \eta \varsigma^{\cdot} \iota \zeta^{\prime} \times \iota \beta^{\prime} \cdot \phi . v \xi \theta^{\prime} \cdot \gamma \rho . \iota \gamma^{\prime}$. 
115

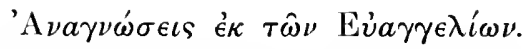

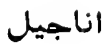

$\mathrm{X} \alpha^{\prime} \rho \tau \eta \varsigma^{\cdot} \iota \zeta^{\prime} \times \iota \gamma^{\prime} \cdot \phi . \sigma o \eta^{\prime} \cdot \gamma \rho . \iota \delta^{\prime}$.

116

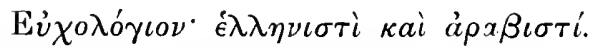

كتاب صلوات

$\mathrm{X} \alpha \rho \tau \eta s^{\cdot} \kappa a^{\prime} \times \iota \epsilon^{\prime} \quad \phi . \rho 9 \eta^{\prime} \gamma \rho . \iota \zeta^{\prime}$.

\section{7}

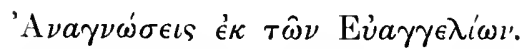

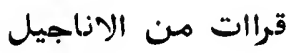

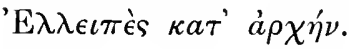

$\mathrm{X} \alpha \dot{\alpha} \rho \tau \eta \varsigma^{\cdot} \kappa \gamma^{\prime} \times \iota \varsigma^{\prime} \cdot \phi . \rho \lambda \theta^{\prime} \cdot \gamma \rho . \iota \epsilon^{\prime}$. M. X.,$a \rho \lambda a^{\prime}$.

118

قراات من الاناجيل تقرا في طول السنة

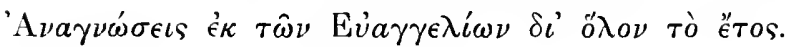

$\mathrm{X} a ́ \rho \tau \eta \varsigma^{\cdot} \kappa \epsilon^{\prime} \times \iota \zeta^{\prime} \cdot \phi . \tau \lambda \zeta^{\prime} \cdot \gamma \rho . \kappa \gamma^{\prime}$.

119

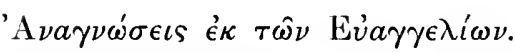

قراات من الاناجيل

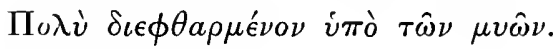

$\mathrm{X} a \rho \tau \eta \varsigma^{*} \kappa \delta^{\prime} \times \iota \zeta^{\prime} \phi . v \lambda \varsigma^{\prime} \gamma \rho . \iota \epsilon^{\prime}$.

\section{0}

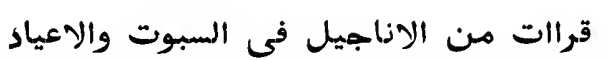

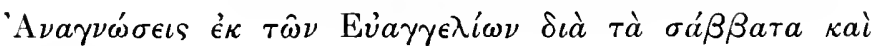
tàs éoptás.

$\mathrm{X} a ́ \rho \tau \eta \varsigma^{\cdot} \kappa a^{\prime} \times \iota \gamma^{\prime} \phi . \sigma \lambda a^{\prime} \gamma \rho . \iota \gamma^{\prime}$.

G. A. M. 


\section{1}

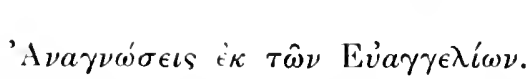

Х $a^{\prime} \rho \tau \eta \varsigma^{\cdot} \kappa \eta^{\prime} \times \kappa^{\prime} \phi \phi . \tau \xi^{\prime} \cdot \gamma \rho . \quad \eta^{\prime}$. M. X. $a \phi \lambda \varsigma^{\prime}$.

122

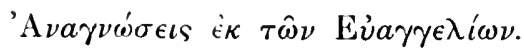

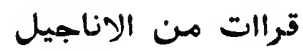

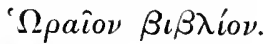

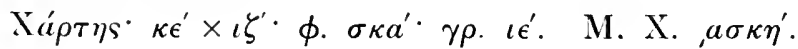

\section{3}

'A

$$
\text { قرال من الاناجيل }
$$

$\mathrm{Xá} \rho \tau \eta \varsigma^{*} \kappa \beta^{\prime} \times \iota \gamma^{\prime} \phi . \rho 9 \gamma^{\prime} \gamma \rho . \iota \epsilon^{\prime}$.

\section{4}

$$
\text { قراات للاعياد من الاناجيل }
$$

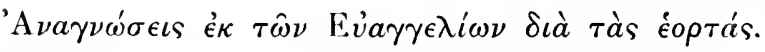

$\mathrm{X} \alpha \rho \tau \eta \varsigma^{\cdot} \kappa \alpha^{\prime} \times \iota \delta^{\prime} \phi . \sigma \lambda \epsilon^{\prime} \hat{\omega} \nu \lambda \epsilon^{\prime} \dot{\epsilon} \lambda \lambda \eta \nu \iota \sigma \tau i^{\cdot} \gamma \rho . \iota \delta^{\prime}$.

\section{5}

'А

$$
\text { قرالت من الاناجيل }
$$

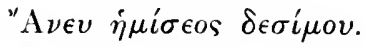

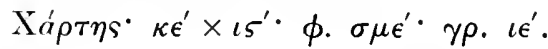

\section{6}

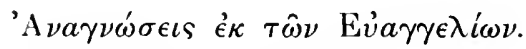

$$
\text { قراات من الاناجيل }
$$

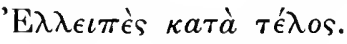

$\mathrm{X} a ́ \rho \tau \eta s^{\cdot} \iota \eta^{\prime} \times \iota \gamma^{\prime} \phi . \tau \iota \delta^{\prime} \cdot \gamma \rho . \iota \beta^{\prime}$.

\section{7}

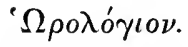

Хáp $\tau \varsigma^{\cdot} \kappa \eta^{\prime} \times \kappa a^{\prime} \cdot \phi . \sigma \lambda \eta^{\prime}$. 


\section{8}

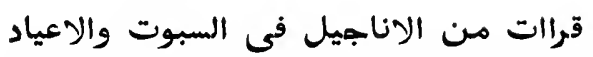

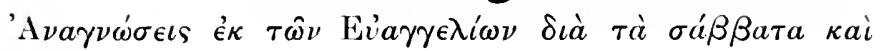
tàs éoprás.

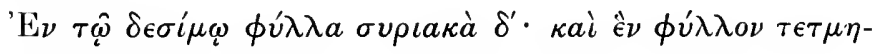

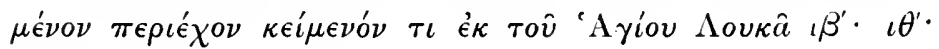

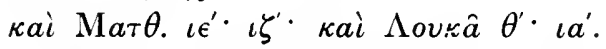

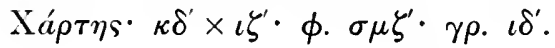

\section{9}

\section{قراات دن الاناجيل التي تقرا في طول السنة}

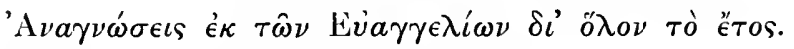

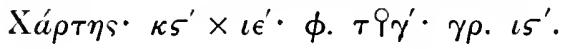

\section{0}

$$
\text { قوانين. قرات من الاناجيل }
$$

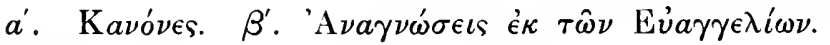
$\mathrm{X} a ́ \rho \tau \eta s^{\cdot} \kappa \theta^{\prime} \times \iota \zeta^{\prime} \cdot \phi \cdot \rho \lambda \gamma^{\prime} \cdot \gamma \rho \cdot \iota \zeta^{\prime}$.

\section{1}

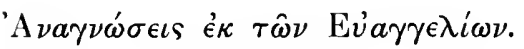

$$
\text { قراات من الاناجيل }
$$

$\mathrm{X} a ́ \rho \tau \eta \varsigma^{\cdot} \iota \zeta^{\prime} \times \iota \beta^{\prime} \cdot \phi \cdot \rho \iota \epsilon^{\prime} \cdot \gamma \rho \cdot \iota \epsilon^{\prime}$.

\section{2}

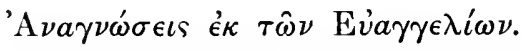

$$
\text { قراات من الاناجيل }
$$

$\mathrm{X} a \dot{\rho} \tau \eta \varsigma^{\cdot} \kappa a^{\prime} \times \iota \delta^{\prime} \cdot \phi . \phi \iota \varsigma^{\prime} \gamma \rho . \iota \epsilon^{\prime}$.

\section{3}

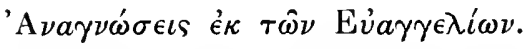

$$
\text { قراات من الاناجيل }
$$

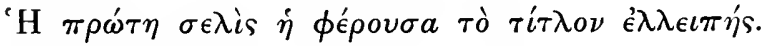

$\mathrm{X} a ́ \rho \tau \eta s^{*} \iota \eta^{\prime} \times \iota \gamma^{\prime} \phi . \rho \lambda \epsilon^{\prime} \gamma \rho . \iota \delta^{\prime}$. 


\section{4}

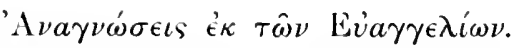

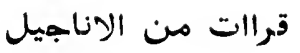

Ха $\rho \tau \eta \varsigma^{\circ} \kappa \delta^{\prime} \times \imath \varsigma^{\prime} \phi . \sigma o a^{\prime} \gamma \rho . \imath \eta^{\prime}$. M. X.,$a \nu \gamma^{\prime}$.

\section{5}

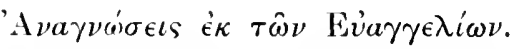

$$
\text { قرالت من الاناجيل }
$$

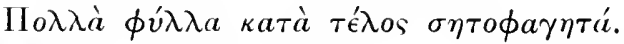

Ха́ $\rho \tau \eta \varsigma^{\cdot} \kappa \eta^{\prime} \times \kappa a^{\prime} \phi . \sigma \eta^{\prime} \gamma \rho . \kappa \delta^{\prime}$. M. X. , $a \phi \imath^{\prime} \eta^{\prime}$.

\section{6}

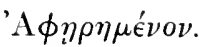

\section{7}

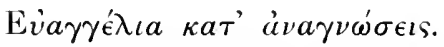

$$
\text { ازاجيل }
$$

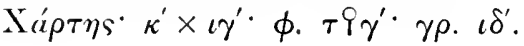

\section{8}

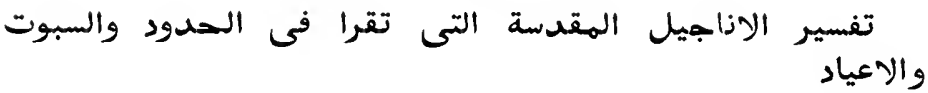

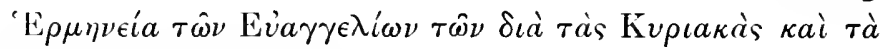
бáßßата каi тàs éopтás.

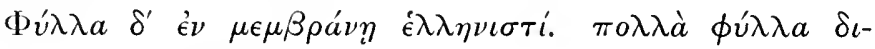
$\epsilon \sigma \pi a \sigma \mu \epsilon^{\prime} \nu^{\prime} \kappa a \tau a ̀ ~ \tau \epsilon^{\prime} \lambda o s$.

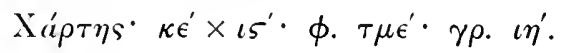

\section{9}

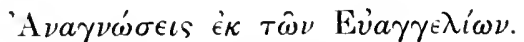

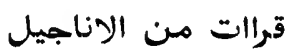

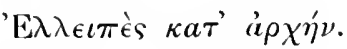

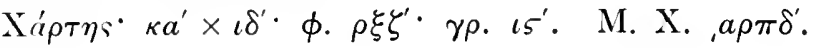




\section{0}

\section{قرالت من الاناجيل · قسمة تكوين}

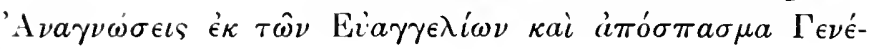
$\sigma \epsilon \omega s$.

'H $\pi \rho \dot{\omega} \tau \eta \sigma \epsilon \lambda$ is $\epsilon \kappa \tau \mu \eta \theta \epsilon \hat{\imath} \sigma a$.

Ха́ $\rho \tau \eta s^{\cdot} \iota \varsigma^{\prime} \times \iota \beta^{\prime} \cdot \phi . \rho^{\prime} \gamma \rho . \iota \gamma^{\prime}$.

\section{1}

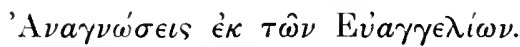

$$
\text { قرالت من الاناجيل }
$$

Tò $\ddot{\eta} \mu \iota \sigma v \sigma v \rho \iota \sigma \tau i ́$.

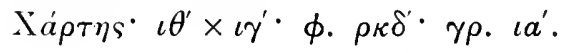

\section{2}

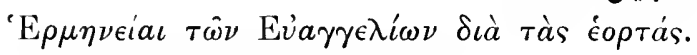

$\mathrm{X} a \rho \tau \eta \varsigma^{\cdot} \kappa a^{\prime} \times \iota \eta^{\prime} \phi . \quad \tau \kappa \gamma^{\prime} \gamma \rho . \iota \gamma^{\prime}$.

\section{3}

$\mathrm{K} \eta \rho u ́ \gamma \mu a \tau a$.

$$
\text { كتاب مواعظ }
$$

$\mathrm{X} a \rho \tau \eta \varsigma^{\cdot} \iota \eta^{\prime} \times \iota \delta^{\prime} \cdot \phi . \rho \uparrow \beta^{\prime} \gamma \rho, \iota \theta^{\prime}$.

\section{4}

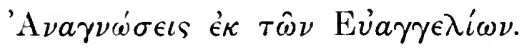

$$
\text { قراات من الاناجيل }
$$

$\mathrm{X} a ́ \rho \tau \eta \varsigma^{\cdot} \iota \zeta^{\prime} \times \iota \gamma^{\prime} \phi . \rho \epsilon^{\prime} \gamma \rho . \iota \gamma^{\prime}$.

\section{5}

$\mathrm{K} \eta \rho \dot{\gamma} \mu a \tau a$.

مقالت

$\mathrm{X} a ́ \rho \tau \eta \varsigma^{\cdot} \iota \theta^{\prime} \times \iota \gamma^{\prime} \phi . \rho \xi \beta^{\prime} \gamma \rho . \iota \epsilon^{\prime}$.

\section{6}

$\mathrm{K} \eta \rho \dot{\gamma} \mu a \tau a$.

$$
\text { كتاب مقالات }
$$

$\mathrm{X} a ́ \rho \tau \eta \varsigma^{*} \kappa a^{\prime} \times \iota \delta^{\prime} \cdot \phi . \quad \nu \delta^{\prime} \cdot \gamma \rho . \eta^{\prime}$. 


\section{7}

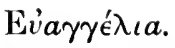

اناجيل

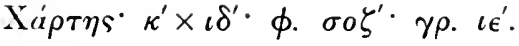

\section{8}

$\mathrm{K} \eta \rho \dot{\gamma} \mu a \tau a$.

مقالات

$\mathrm{X} a ́ \rho \tau \eta \varsigma^{*} \iota \eta^{\prime} \times \iota \gamma^{\prime} \cdot \phi . \sigma o \varsigma^{\prime} \cdot \gamma \rho . \iota \eta^{\prime}$.

149

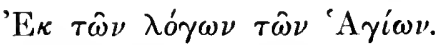

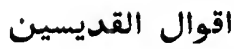

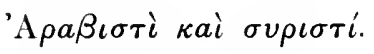

$\mathrm{X} a ́ \rho \tau \eta \varsigma^{\cdot} \iota \theta^{\prime} \times \imath \gamma^{\prime} \cdot \phi$. i $\gamma^{\prime} \cdot \gamma \rho . \imath \beta^{\prime}$.

150

فصول الاناجيل والرسالات

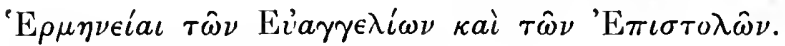

Ха́ $\rho \tau \eta \varsigma^{\cdot} \kappa \delta^{\prime} \times \iota \zeta^{\prime} \cdot \phi . \tau \iota^{\prime} \cdot \gamma \rho . \iota \eta^{\prime}$.

\section{1}

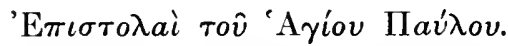

رسالات بولص القديس

$M_{\epsilon \mu \beta \rho a ́ \nu \eta} \kappa \epsilon^{\prime} \times \iota \eta^{\prime} \cdot \phi . \sigma \xi \eta^{\prime} \cdot \gamma \rho . \iota \eta^{\prime}$.

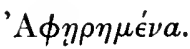

\section{$\left.\begin{array}{l}152 \\ 153\end{array}\right\}$}

\section{4}

$\Pi \rho a^{\prime} \xi \epsilon \varsigma \tau \hat{\omega} \nu$ 'A $\pi \sigma \sigma \tau o ́ \lambda \omega \nu$.

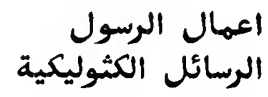

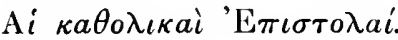

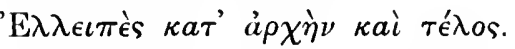

М $\epsilon \mu \beta \rho a^{\prime} \eta^{\prime} \iota \eta^{\prime} \times \iota \beta^{\prime} \cdot \phi . \rho \mu a^{\prime} \gamma \rho . \kappa^{\prime}$. 



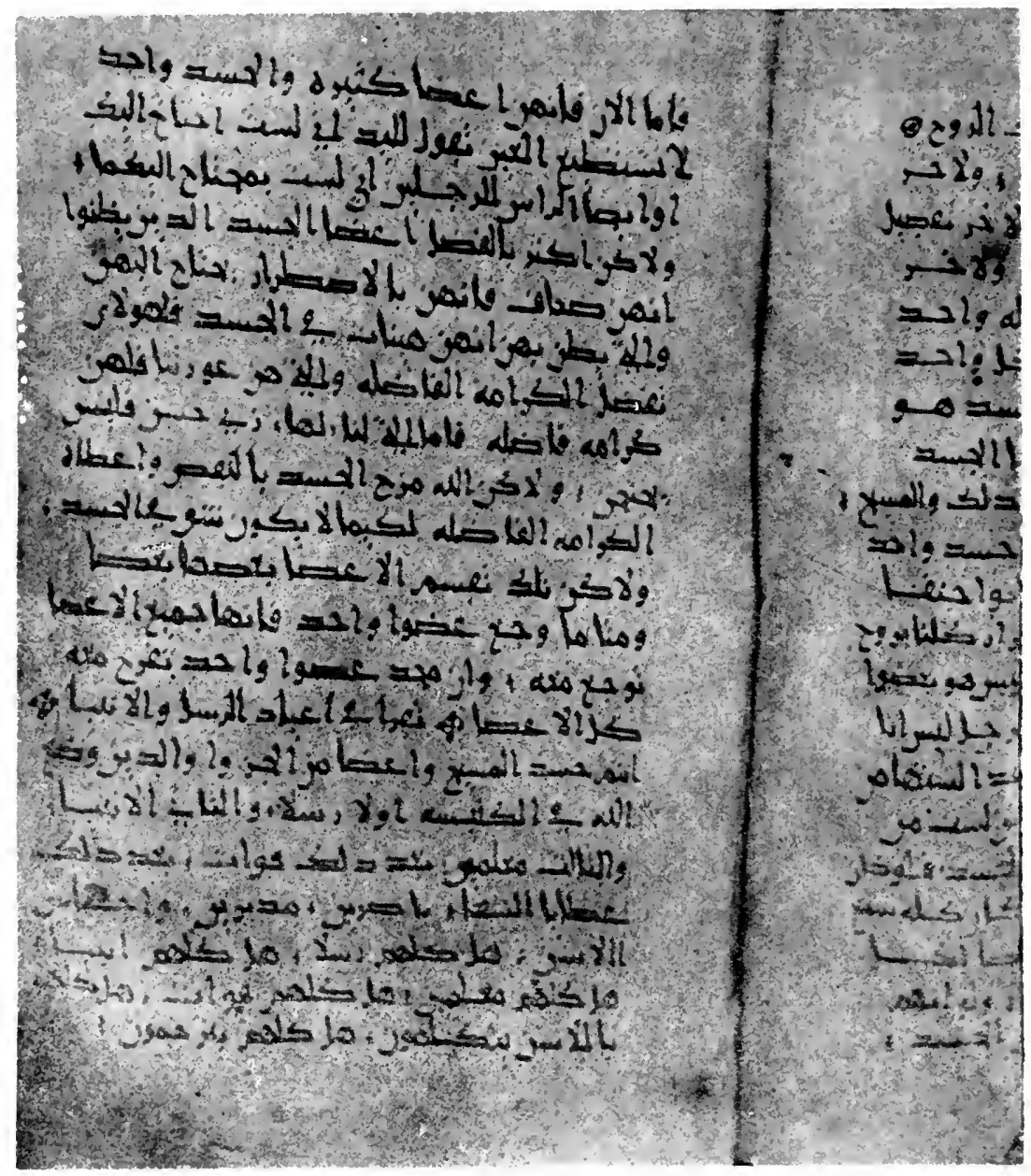

1 COKINTHIANS XI ?O 30

NO 155

Eraph M. Mrs liewlis) 


\section{5}

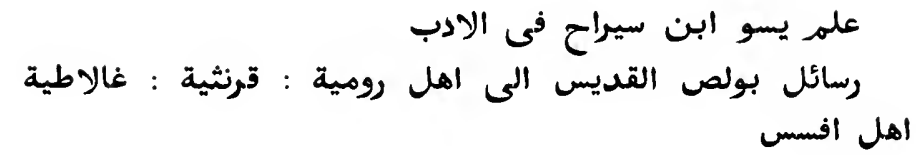

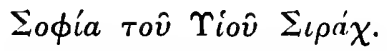

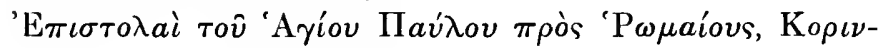

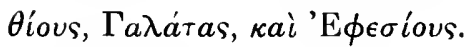

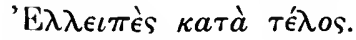

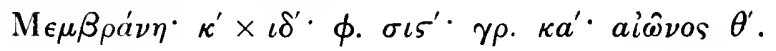

\section{6}

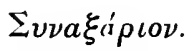

$\mathrm{X} a ́ \rho \tau \eta \varsigma^{\cdot} \kappa^{\prime} \times \iota \delta^{\prime} \cdot \phi . \sigma \mu \delta^{\prime} \cdot \gamma \rho . \iota \eta^{\prime}$.

'A $\phi \eta \rho \eta \mu \in ́ \nu o \nu$.

\section{7}

\section{8}

اعمال الرسل : رسائل القديس بولص : الرسائل الكثوليكية

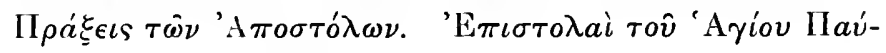

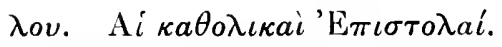

$\mathrm{X} a ́ \rho \tau \eta \varsigma^{\cdot} \kappa \varsigma^{\prime} \times \kappa^{\prime} \phi . \rho o \delta^{\prime} \gamma \rho . \kappa a^{\prime}$.

\section{9}

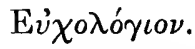

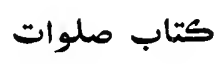

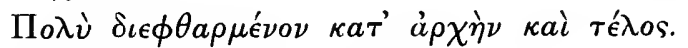

$\mathrm{X} a ́ \rho \tau \eta \varsigma^{\cdot} \kappa^{\prime} \times \iota \gamma^{\prime} \phi . \rho \xi \zeta^{\prime} \gamma \rho . \iota \zeta^{\prime}$.

\section{0}

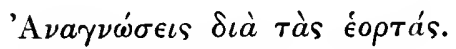

$$
\text { قرات لNعياد }
$$

$\mathrm{X} a ́ \rho \tau \eta \varsigma^{\cdot} \kappa^{\prime} \times \iota \delta^{\prime} \cdot \phi . \sigma \iota \eta^{\prime} \cdot \gamma \rho . \kappa a^{\prime}$. 
161

رسائل القديس بولص : ابركسس التلاهيذ : الرسائل الكثوليكية

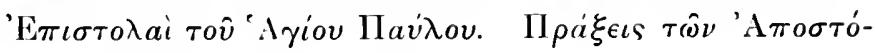

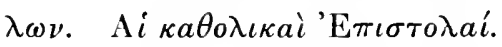

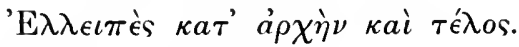

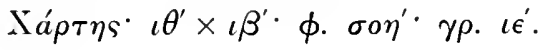

162

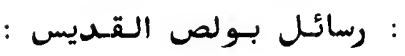

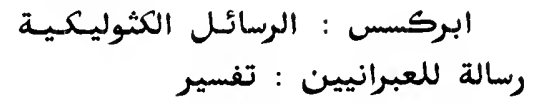

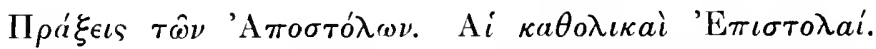

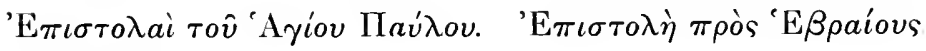

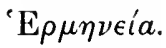

$\Pi o \lambda \dot{v} \delta \iota \epsilon \sigma \pi a \sigma \mu \dot{\epsilon}_{\nu o \nu}$

$\mathrm{X} a ́ \rho \tau \eta \varsigma^{*} \lambda a^{\prime} \times \kappa \gamma^{\prime} \cdot \phi . \sigma \rho \epsilon^{\prime} \cdot \gamma \rho . \iota \theta^{\prime}$.

\section{3}

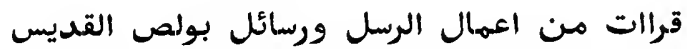

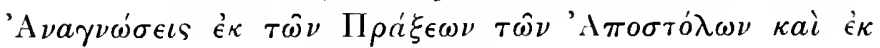

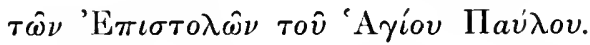

Ха́ $\rho \tau \eta \varsigma^{\prime} \kappa \epsilon^{\prime} \times \iota \epsilon^{\prime} \cdot \phi \cdot \rho \lambda \varsigma^{\prime} \cdot \gamma \rho . \iota \zeta^{\prime}$.

\section{4}

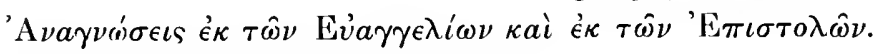
$\mathrm{X} a ́ \rho \tau \eta \varsigma^{\cdot} \kappa \varsigma^{\prime} \times \iota \zeta^{\prime} \cdot \phi . \tau \nu \epsilon^{\prime} \cdot \gamma \rho . \kappa a^{\prime}$.

\section{5}

رسائل القديس بولص : الرسائل الكثوليكية : مزامير

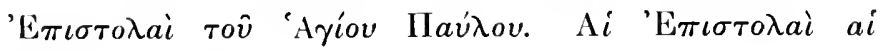

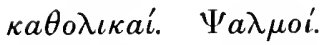

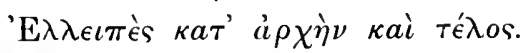

$\mathrm{X} a ́ \rho \tau \eta s^{\cdot} \kappa \gamma^{\prime} \times \iota \zeta^{\prime} \cdot \phi . \rho \xi^{\prime} \cdot \gamma \rho \cdot \iota \gamma^{\prime}$. 


\section{6}

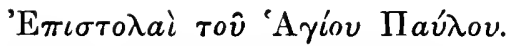

$$
\text { رسائل القديس بولص }
$$

$\mathrm{X} a ́ \rho \tau \eta \varsigma^{\cdot} \iota \delta^{\prime} \times \theta^{\prime} \cdot \phi . \sigma \xi \beta^{\prime} \cdot \gamma \rho . \iota a^{\prime}$.

\section{7}

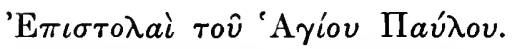

$$
\text { رسائل القديس بولص }
$$

$\mathrm{X} a^{\prime} \rho \tau \eta s^{\cdot} \kappa a^{\prime} \times \imath \epsilon^{\prime} \cdot \phi \cdot \rho \mu^{\prime} \cdot \gamma \rho . \iota 5^{\prime}$.

168

$$
\text { اعمال الرسل ورسائل القديس بولص }
$$

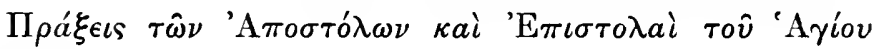
Паú̃ov.

$\mathrm{X} a \dot{\rho} \tau \eta \varsigma^{\cdot} \iota \zeta^{\prime} \times \iota \gamma^{\prime} \cdot \phi . \phi^{\prime} \cdot \gamma \rho . \iota \gamma^{\prime}$.

\section{9}

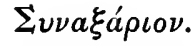

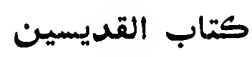

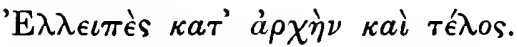

$\mathrm{X} a ́ \rho \tau \eta \varsigma^{\cdot} \kappa a^{\prime} \times \iota \gamma^{\prime} \cdot \phi . v \iota a^{\prime} \cdot \gamma \rho . \iota \epsilon^{\prime}$.

\section{0}

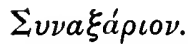

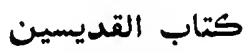

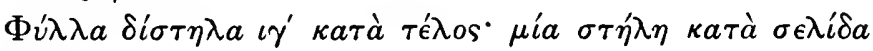
$\dot{\epsilon} \lambda \lambda \eta \nu \iota \sigma \tau i$.

$\mathrm{X} a ́ \rho \tau \eta s^{\bullet} \kappa \eta^{\prime} \times \kappa \beta^{\prime} \cdot \phi . \sigma \eta^{\prime} \cdot \gamma \rho . \kappa \beta^{\prime}$.

\section{1}

$\Pi_{\rho a ́}{ }_{\epsilon \iota \varsigma} \tau \hat{\omega} \nu$ 'A اعهال الرسل ورسائل $\sigma \epsilon \iota s$.

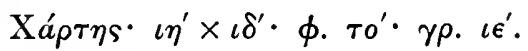

G. A. M. 


\section{2}

اعهال الرسل ورسائل

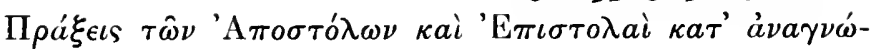
$\sigma \in \iota$.

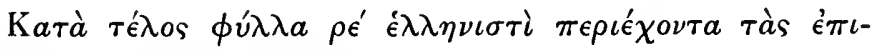

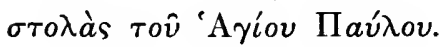

$\mathrm{X} a ́ \rho \tau \eta s^{\cdot} \iota \theta^{\prime} \times \iota \beta^{\prime} \phi \phi . \sigma o a^{\prime} \gamma \rho . \iota \delta^{\prime}$.

\section{3}

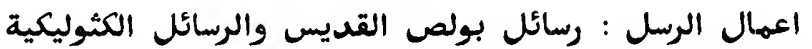

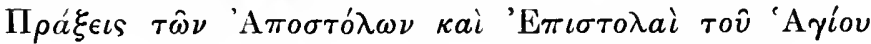

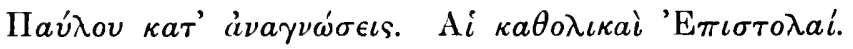

$\mathrm{X} a ́ \rho \tau \eta s^{\cdot} \kappa a^{\prime} \times \iota \delta^{\prime} \cdot \phi . \sigma o s^{\prime} \gamma \rho . \iota \theta^{\prime}$.

\section{4}

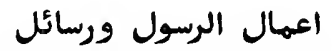

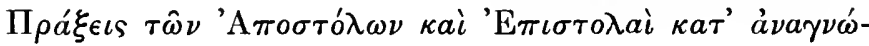
$\sigma \in \iota \varsigma$.

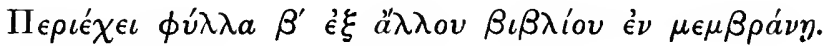
$\mathrm{X} a ́ \rho \tau \eta \varsigma^{\cdot} \kappa \epsilon^{\prime} \times \iota \zeta^{\prime} \cdot \phi . \rho \pi \epsilon^{\prime} \gamma \rho . \iota \eta^{\prime}$.

175

$\Sigma v \nu a \xi a ́ \rho \iota v$.

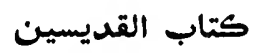

$\mathrm{X} \alpha \dot{\rho} \rho \eta \varsigma^{\cdot} \kappa \theta^{\prime} \times \kappa^{\prime} \cdot \phi . \rho \pi \gamma^{\prime} \gamma \rho . \iota \theta^{\prime}$.

\section{6}

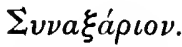

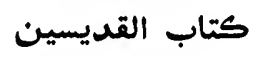

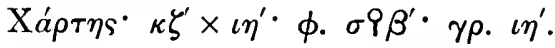

\section{7}

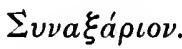

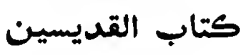

$\mathrm{X} a \dot{\rho} \tau \eta \varsigma^{\cdot} \iota \eta^{\prime} \times \iota \delta^{\prime} \phi \phi . \tau \mu^{\prime} \gamma \rho . \iota \beta^{\prime}$.

\section{8}

$\Sigma v \nu a \xi a ́ \rho \iota \nu$.

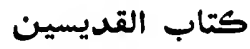

$\mathrm{X} a ́ \rho \tau \eta \varsigma^{*} \kappa^{\prime} \times \iota \delta^{\prime} \cdot \phi . \sigma \kappa \eta^{\prime} \gamma \rho . \iota \gamma^{\prime}$. 
179

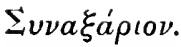

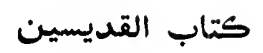

$\Pi o \lambda \nu \dot{~} \delta \iota \phi \theta \theta \rho \mu \epsilon^{\prime} \nu \nu \nu$.

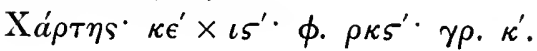

180

$\Psi a \lambda \tau \eta ́ \rho \iota ⿻ \nu$.

كتاب المززامير

$\mathrm{X} a \rho \rho \eta s^{\cdot} \iota \zeta^{\prime} \times \iota \gamma^{\prime} \phi . \rho \lambda \epsilon^{\prime} \gamma \rho . \iota \beta^{\prime}$.

\section{1}

$\Psi a \lambda \tau \eta \dot{\rho} \iota \nu \nu$.

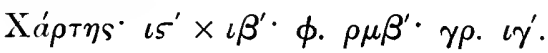

182

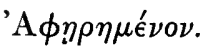

\section{3}

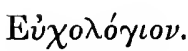

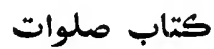

$\mathrm{X} a ́ \rho \tau \eta s^{\cdot} \iota \zeta^{\prime} \times \iota \beta^{\prime} \phi . \rho \nu \theta^{\prime} \gamma \rho . \iota \gamma^{\prime}$.

\section{4}

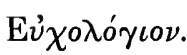

$$
\text { كتّاب صلوات }
$$

X $\alpha^{\prime} \rho \tau \eta s^{\cdot} \iota \gamma^{\prime} \times \eta^{\prime} \phi . \rho \xi 5^{\prime} \cdot \gamma \rho . \eta^{\prime}$.

\section{5}

$\Psi a \lambda \tau \eta \dot{\rho} \iota$ ข.

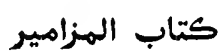

$\mathrm{X} a ́ \rho \tau \eta s^{\cdot} \iota \zeta^{\prime} \times \iota \gamma^{\prime} \phi \cdot \rho \pi \delta^{\prime} \cdot \gamma \rho . \iota a^{\prime}$.

\section{6}

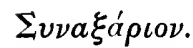

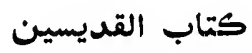

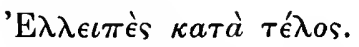

$\mathrm{X} a ́ \rho \tau \eta s^{*} \iota \zeta^{\prime} \times \iota a^{\prime} \cdot \phi . \rho \lambda^{\prime} \cdot \gamma \rho . \iota a^{\prime}$. 


\section{7}

$\Psi a \lambda \tau \eta ́ p \iota ⿻ \nu$.

كتاب الهزامير

$\mathrm{X} a \rho \tau \eta s^{\cdot} \iota \delta^{\prime} \times \iota^{\cdot} \phi .9 a^{\prime} \gamma \rho . \iota a^{\prime}$.

188

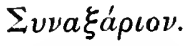

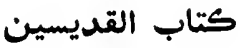

$\Pi o \lambda i \quad \delta \iota \epsilon \phi \theta a \rho \mu \epsilon^{\prime} \nu o \nu$.

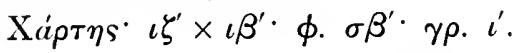

189

$\Psi a \lambda \tau \dot{p} \rho \iota 0 \nu$.

كتاب الهزامير

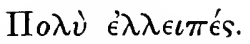

$\mathrm{X} \alpha \dot{\rho} \rho \tau \eta s^{\cdot} \iota \zeta^{\prime} \times \iota \beta^{\prime} \cdot \phi . \sigma \mu^{\prime} \gamma \rho . \iota \beta^{\prime}$.

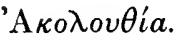

190

$\mathrm{X} a \dot{\rho} \tau \eta s^{\cdot} \iota \gamma^{\prime} \times \imath^{\prime} \phi . \sigma \kappa \varsigma^{\prime} \gamma \rho . \iota^{\prime}$.

\section{1}

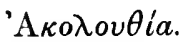

$\mathrm{X} a \dot{\rho} \tau \eta s^{\cdot} \iota \gamma^{\prime} \times \theta^{\prime} \cdot \phi \cdot \rho \pi \delta^{\prime} \cdot \gamma \rho . \iota \eta^{\prime}$.

192

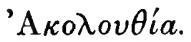

$\mathrm{X} a ́ \rho \tau \eta s^{*} \iota \delta^{\prime} \times \iota^{\prime} \cdot \phi . \rho \kappa \zeta^{\prime} \cdot \gamma \rho . \iota^{\prime}$.

193

قانون مديح السيدة والدة الاهنا يقال فى هياكل النصرانية

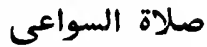

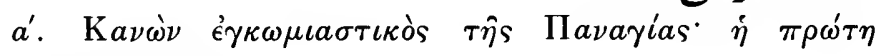

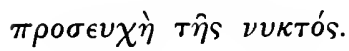

$\mathrm{X} a^{\prime} \rho \tau \eta \varsigma^{\cdot} \iota \zeta^{\prime} \times \iota \beta^{\prime} \cdot \phi . \sigma \kappa \alpha^{\prime} \cdot \gamma \rho . \iota \beta^{\prime}$.

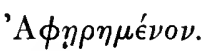

\section{4}




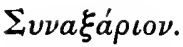

\section{5}

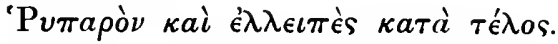

$\mathrm{X} a \rho \tau \eta s^{\cdot} \iota \eta^{\prime} \times \iota \delta^{\prime} \cdot \phi . \rho \mu a^{\prime} \gamma \rho . \iota \delta^{\prime}$.

\section{6}

Eủxo入óyıv.

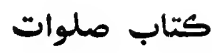

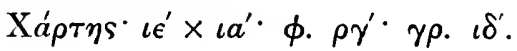

\section{7}

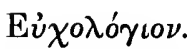

كتاب صلوات

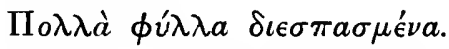

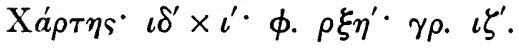

\section{8}

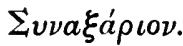

$\mathrm{X} a ́ \rho \tau \eta \varsigma^{\cdot} \iota \theta^{\prime} \times \iota \delta^{\prime} \cdot \phi . \sigma \iota \delta^{\prime} \cdot \gamma \rho . \iota \epsilon^{\prime}$.

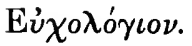

\section{9}

$\mathrm{X} a ́ \rho \tau \eta \varsigma^{*} \kappa a^{\prime} \times \iota \epsilon^{\prime} \cdot \phi . \rho \lambda \delta^{\prime} \cdot \gamma \rho . \kappa a^{\prime}$.

\section{0}

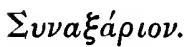

$\mathrm{X} a^{\prime} \rho \tau \eta \varsigma^{\cdot} \kappa^{\prime} \times \iota \gamma^{\prime} \phi . \rho \circ \beta^{\prime} \cdot \gamma \rho . \iota \zeta^{\prime}$.

\section{1}

$\Psi a \lambda \tau \eta^{\prime} \rho$ เov.

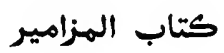

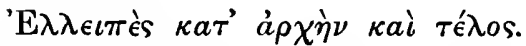

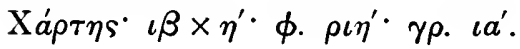

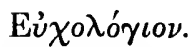

\section{2}

$\mathrm{X} a ́ \rho \tau \eta \varsigma^{*} \iota \eta^{\prime} \times \iota \beta^{\prime} \cdot \phi . \rho \mu \theta^{\prime} \cdot \gamma \rho . \iota \gamma^{\prime}$. 


\section{3}

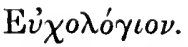

كتاب صلوات

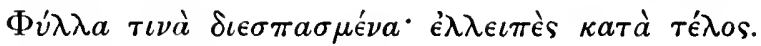

$\mathrm{X} a \dot{\rho} \rho \tau \eta \varsigma^{\cdot} \iota \beta^{\prime} \times \eta^{\prime} \cdot \phi . \rho \mu \varsigma^{\prime} \cdot \gamma \rho . \iota^{\prime}$.

'АколоvӨía.

\section{4}

$\mathrm{X} \alpha \dot{\rho} \rho \eta \varsigma^{*} \iota \eta^{\prime} \times \iota \gamma^{\prime} \cdot \phi \cdot \rho \iota \zeta^{\prime} \cdot \gamma \rho . \iota^{\prime}$.

\section{5}

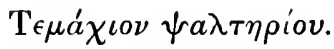

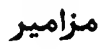

$\mathrm{X} a ́ \rho \tau \eta \varsigma^{\prime} \kappa^{\prime} \times \iota \gamma^{\prime} \cdot \phi . \xi a^{\prime} \cdot \gamma \rho . \iota \epsilon^{\prime}$.

\section{6}

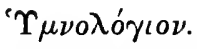

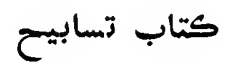

$\mathrm{X} a ́ \rho \tau \eta \varsigma^{\cdot} \iota \gamma^{\prime} \times \theta^{\prime} \cdot \phi . \rho \mu \beta^{\prime} \cdot \gamma \rho . \iota^{\prime}$.

\section{7}

Eủxo入óyıov.

$$
\text { كتاب صلوات }
$$

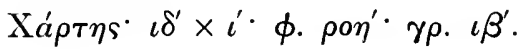

\section{8}

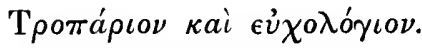

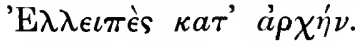

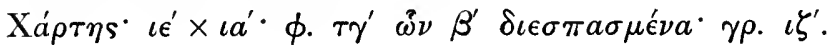

209

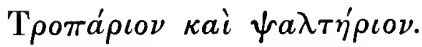

اطروبارية ومزامير

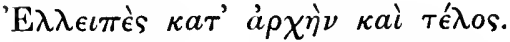

$\mathrm{X} a ́ \rho \tau \eta \varsigma^{\cdot} \iota \zeta^{\prime} \times \iota \beta^{\prime} \cdot \phi . \rho \uparrow \zeta^{\prime} \cdot \gamma \rho . \iota \beta^{\prime}$. 


\section{0}

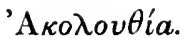

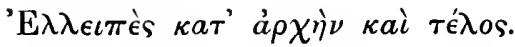

$\mathrm{X} a ́ \rho \tau \eta \varsigma^{\cdot} \kappa^{\prime} \times \iota \gamma^{\prime} \phi . \rho o \gamma^{\prime} \gamma \rho . \iota \delta^{\prime}$.

\section{1}

$\sum v v a \xi a ́ \rho \imath \nu$.

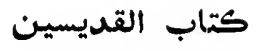

$\mathrm{X} a ́ \rho \tau \eta \varsigma^{*} \kappa^{\prime} \times \iota \gamma^{\prime} \phi .95^{\prime} \gamma \rho . \iota \gamma^{\prime}$.

\section{2}

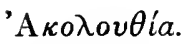

$\mathrm{X} a \dot{\rho} \tau \eta \varsigma^{\cdot} \iota \zeta^{\prime} \times \iota \gamma^{\prime} \phi . \rho \iota \epsilon^{\prime} \gamma \rho . \iota a^{\prime}$.

\section{3}

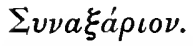

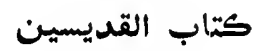

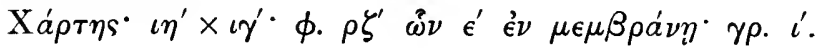

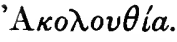

\section{4}

$\mathrm{X} a ́ \rho \tau \eta \varsigma^{\cdot} \kappa^{\prime} \times \iota \gamma^{\prime} \phi . \rho \kappa \zeta^{\prime} \cdot \gamma \rho . \iota \delta^{\prime}$.

\section{5}

$\Psi a \lambda \tau \eta \dot{\rho} \iota$ ข.

$$
\text { كتاب الهزامير }
$$

$\mathrm{X} a \dot{\rho} \tau \eta s^{\cdot} \iota \epsilon^{\prime} \times \iota a^{\prime} \cdot \phi . \rho \xi a^{\prime} \cdot \gamma \rho . \iota \beta^{\prime}$.

\section{6}

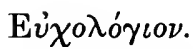

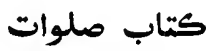

$\Pi o \lambda \dot{v} \epsilon \lambda \lambda \epsilon \iota \pi \hat{s}$.

Ха́

\section{7}

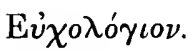

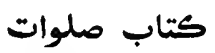

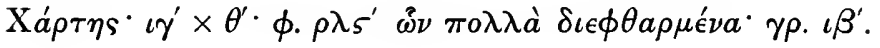




\section{8}

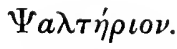

$$
\text { كتاب المزامير }
$$

$\mathrm{X} \alpha \dot{\rho} \tau \eta \varsigma^{\cdot} \iota \zeta^{\prime} \times \iota \gamma^{\prime} \phi . \nu \beta^{\prime} \gamma \rho . \iota a^{\prime}$.

\section{9}

'

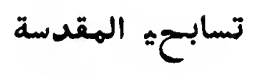

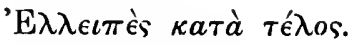

$\mathrm{X} a \rho \tau \eta s^{\cdot} \iota \zeta^{\prime} \times \iota \beta^{\prime} \cdot \phi \cdot \rho \lambda a^{\prime} \gamma \rho . \iota \beta^{\prime}$.

220

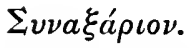

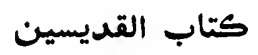

$\mathrm{X} a ́ \rho \tau \eta s^{\cdot} \iota \gamma^{\prime} \times \iota^{\prime} \phi . \sigma \iota \delta^{\prime} \cdot \gamma \rho . \iota^{\prime}$.

\section{1}

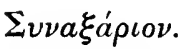

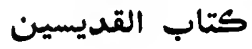

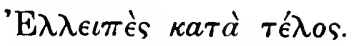

$\mathrm{X} \alpha \dot{\rho} \tau \eta \varsigma^{\circ} \iota \gamma^{\prime} \times \iota^{\prime} \phi . \rho \varsigma^{\prime} \gamma \rho . \iota \beta^{\prime}$.

\section{2}

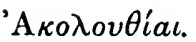

$\mathrm{X} a ́ \rho \tau \eta \varsigma^{\cdot} \iota \epsilon^{\prime} \times \iota^{\prime} \phi . \rho s^{\prime} \dot{\omega} \nu \beta^{\prime} \in \lambda \lambda \eta \nu \iota \sigma \tau \iota^{\prime} \gamma \rho . \iota \delta^{\prime}$.

\section{3}

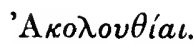

$\mathrm{X} \alpha \rho \tau \eta \varsigma^{\circ} \kappa a^{\prime} \times \iota \varsigma^{\prime} \phi . \rho \kappa \gamma^{\prime} \gamma \rho . \iota \gamma^{\prime}$.

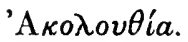

\section{4}

$\mathrm{X} a ́ \rho \tau \eta s^{\bullet} \iota \delta^{\prime} \times \iota^{\prime} \phi . \mu \beta^{\prime} \gamma \rho . \iota \gamma^{\prime}$.

'A $\phi \eta \rho \eta \mu \operatorname{c}_{\nu 0 \nu}$

\section{5}




\section{6}

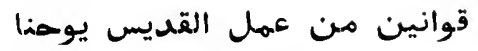

Kavóves tôे 'A yíov 'I

$\mathrm{X} \alpha \dot{\rho} \rho \eta \eta \varsigma^{\cdot} \iota \delta^{\prime} \times \iota^{\prime} \cdot \phi . \sigma \lambda \beta^{\prime} \cdot \gamma \rho . \imath \beta^{\prime}$.

\section{7}

Eủxo入óyıov.

$$
\text { كتاب صلوات }
$$

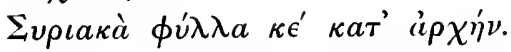

$\mathrm{X} a \dot{\rho} \tau \eta \varsigma^{\bullet} \iota \gamma^{\prime} \times \eta^{\prime}{ }^{\prime} \phi . \sigma \gamma^{\prime} \gamma \rho . \iota \gamma^{\prime}$.

\section{8}

Eủxo入óyıov.

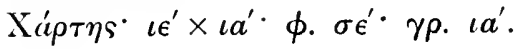

\section{9}

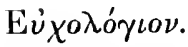

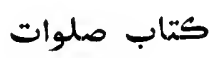

$\mathrm{X} a \rho \rho \eta \varsigma^{\circ} \iota 5^{\prime} \times 1 \beta^{\prime} \cdot \phi . \rho \lambda \eta^{\prime} \hat{\omega} \nu \beta^{\prime} \epsilon \dot{\epsilon} \pi \epsilon \theta \epsilon \tau \iota \kappa a^{\prime} \gamma \rho . \iota \gamma^{\prime}$.

\section{0}

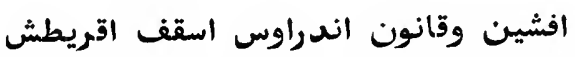

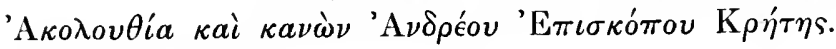
$\mathrm{X} a \dot{\rho} \tau \eta \varsigma^{\cdot} \iota \eta^{\prime} \times \iota \gamma^{\prime} \cdot \phi .9 \varsigma^{\prime} \cdot \gamma \rho . \iota \delta^{\prime}$.

\section{1}

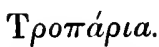

$$
\text { اطروباريات }
$$

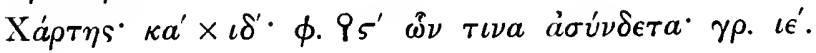

\section{2}

$\Psi a \lambda \tau \dot{\eta} \rho\llcorner o \nu$.

$$
\text { كتماب الهزاهير }
$$

$\mathrm{X} \dot{a} \rho \tau \eta \varsigma^{\cdot} \iota \gamma^{\prime} \times \theta^{\prime} \cdot \phi . \quad \tau \eta^{\prime} \cdot \gamma \rho . \iota \gamma^{\prime}$.

G. A. M. 


\section{3}

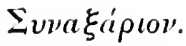

$$
\text { كتاب القديسين }
$$

$\mathrm{X} \dot{\alpha} \rho \tau \eta \varsigma^{*} \kappa^{\prime} \times \imath \delta^{\prime} \cdot \phi . \rho \imath \beta^{\prime} \cdot \gamma \rho .15^{\prime}$.

\section{4}

Kavóves.

$$
\text { قوانين }
$$

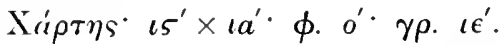

\section{5}

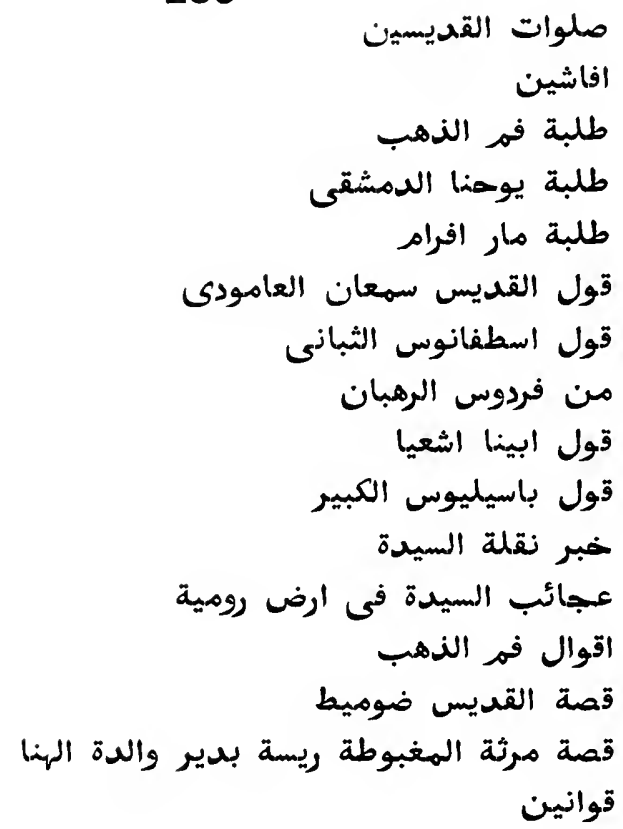

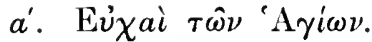

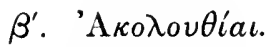

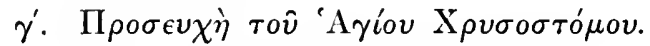

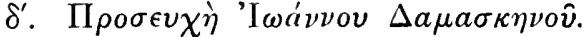

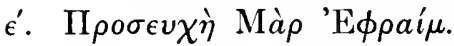

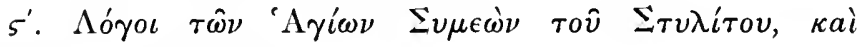

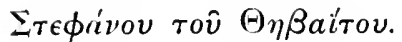




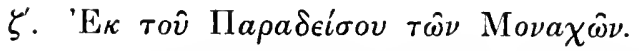

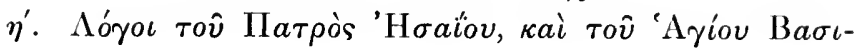
$\lambda \in i o v$.

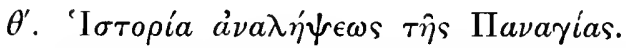

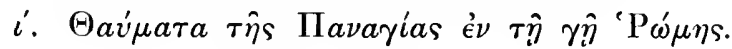

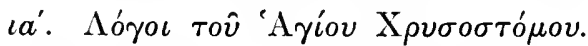

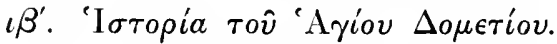

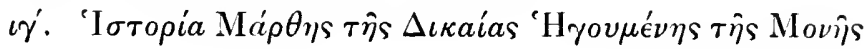

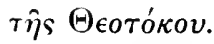

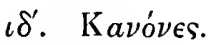

$\mathrm{X} a ́ \rho \tau \eta \varsigma^{\cdot} \iota \epsilon^{\prime} \times \iota^{\prime} \cdot \phi . \sigma \iota a^{\prime} \cdot \gamma \rho . \iota \delta^{\prime}$.

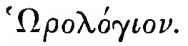

\section{6}

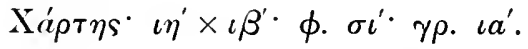

\section{7}

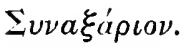

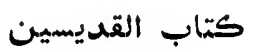

$\mathrm{X} \alpha \rho \tau \eta \varsigma^{\cdot} \iota \epsilon^{\prime} \times \iota a^{\prime} \cdot \phi . \tau \pi \varsigma^{\prime} \gamma \rho . \iota \varsigma^{\prime}$.

\section{8}

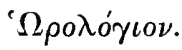

$\mathrm{X} a ́ \rho \tau \eta \varsigma^{\cdot} \iota \zeta^{\prime} \times \iota \gamma^{\prime} \phi . \tau \nu a^{\prime} \gamma \rho . \iota a^{\prime}$.

${ }^{\prime} \Lambda \phi \eta \rho \eta \mu e^{\prime} \nu o \nu$.

\section{9}

\section{0}

'

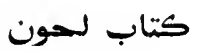

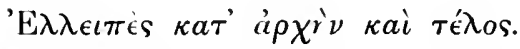

$\mathrm{M}_{\epsilon \mu \beta \rho a ́ \nu \eta} \iota \zeta^{\prime} \times \iota \gamma^{\prime} \phi . \rho \mathrm{P}^{\prime} \cdot \gamma \rho . \iota \beta^{\prime}$.

\section{1}

قانون التوبة عهل الندراوس اسقف جزيرة

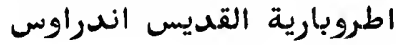

5-2 


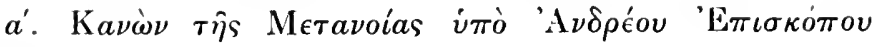
$\Gamma \epsilon \zeta_{i \rho}^{\prime} \eta$ s.

$\beta^{\prime}$. Z $\omega \dot{\omega} \nu \eta \bar{\eta} s \kappa \tau i \sigma \epsilon \omega s$.

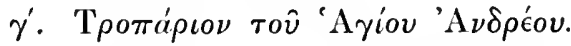

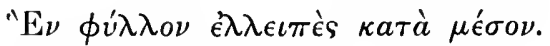

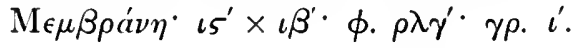

242

تغسير اطروباريات القيامة : النسوة الداهلات العيبب

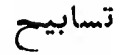

قول القديس يوحنا فم النهاح

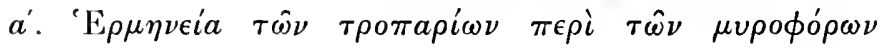
$\gamma \nu \nu a \iota \kappa \hat{\omega} \nu$.

$\beta^{\prime} .{ }^{~ "} \Upsilon \mu \nu \circ$.

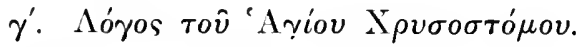

$\mathrm{X} \alpha \rho \tau \eta \varsigma^{\cdot} \iota \zeta^{\prime} \times \imath \beta^{\prime} \cdot \phi . \rho i \epsilon^{\prime} \gamma \rho . \imath \beta^{\prime}$.

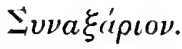

\section{3}

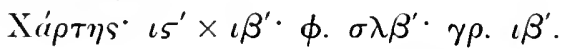

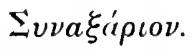

\section{4}

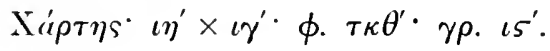

\section{5}

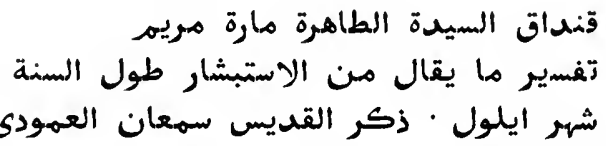

لراس السنة

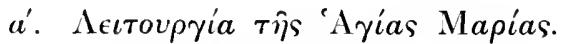

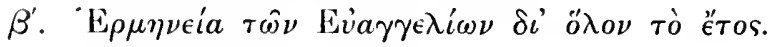

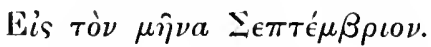

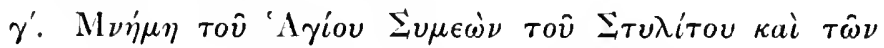

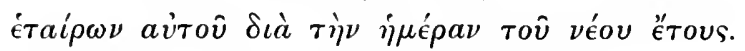

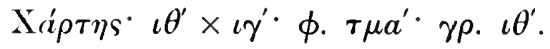




\section{6}

$$
\text { اننفا البارة من سم الهصرية }
$$

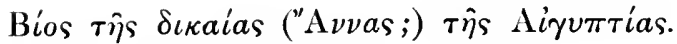

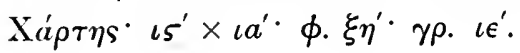

\section{7}

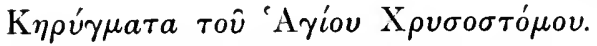

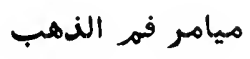

$\mathrm{X} \dot{a} \rho \tau \eta \varsigma^{\cdot} \iota \zeta^{\prime} \times \iota \beta^{\prime} \cdot \phi . \sigma \nu \gamma^{\prime} \cdot \gamma \rho . \iota a^{\prime}$.

\section{8}

\section{قانون خشوعى من عمل الفديس اندراوس اسقف جزيرة}

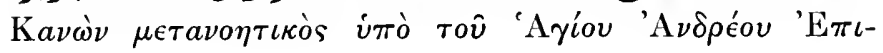

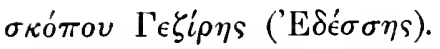

$\mathrm{X} a \dot{\rho} \tau \eta \varsigma^{\cdot} \iota \delta^{\prime} \times \iota a^{\prime} \cdot \phi . \sigma \eta^{\prime} \cdot \gamma \rho . \iota^{\prime}$.

\section{9}

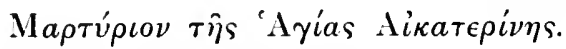

$$
\text { شهادة الهسيهية كترينا }
$$

$\mathrm{X} a ́ \rho \tau \eta \varsigma^{*} \iota \delta^{\prime} \times \iota^{\prime} \gamma \rho . \iota \epsilon^{\prime}$.

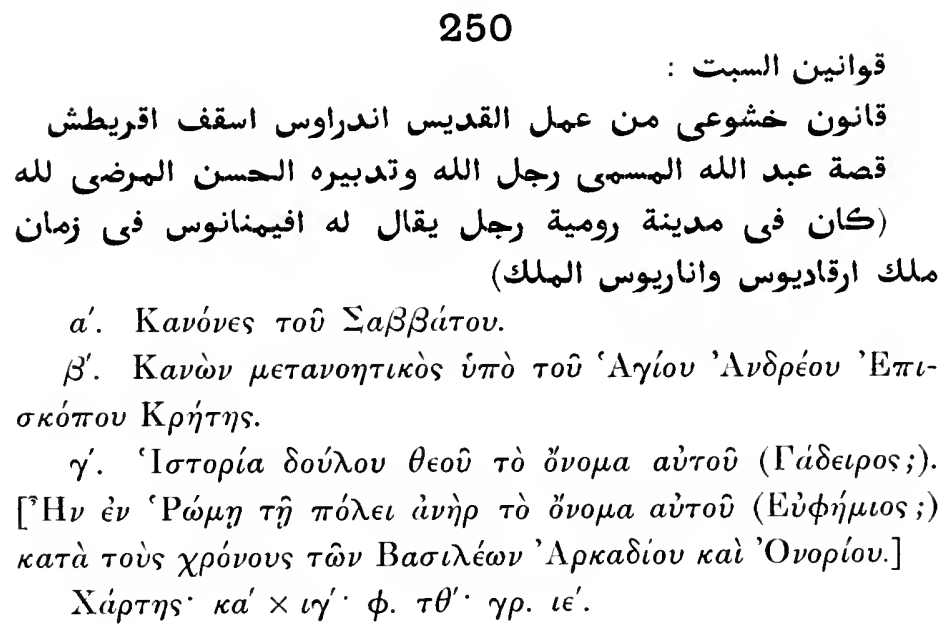




\section{1}

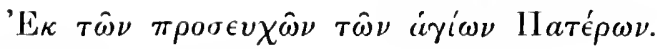

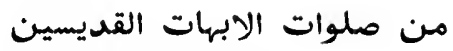

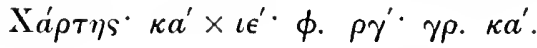

\section{2}

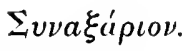

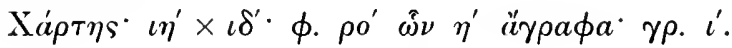

\section{3}

ما يقال من الاستسرار فى ليلة الاحد المعروف بالفريسى

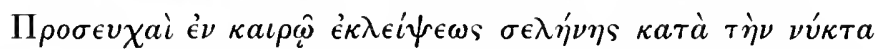

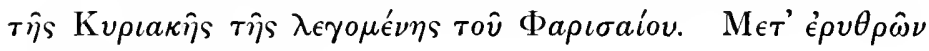
$\dot{\epsilon} \lambda \lambda \eta \nu \iota \kappa \hat{\omega} \nu \sigma \tau o \iota \chi \epsilon i \omega \nu$.

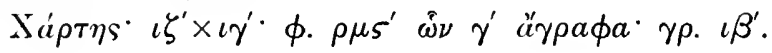

\section{4}

$\Delta \iota \delta a \sigma \kappa a \lambda i a \iota(\dot{a} \gamma \nu \omega \dot{\sigma \tau \tau o v)}$.

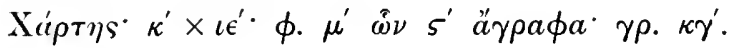

\section{5}

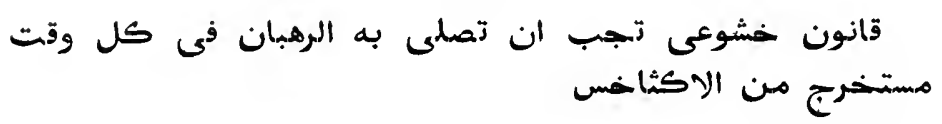

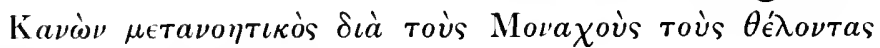
$\grave{\epsilon} \xi \epsilon \lambda \theta \epsilon \hat{\imath} \nu \stackrel{\dot{\epsilon} \kappa}{\tau} \tau o \hat{v} \dot{a} \gamma a \theta 0 \hat{v}$.

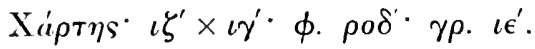

\section{6}

Троти́рıор.

X'́a $\rho \eta \varsigma^{*} \iota \zeta^{\prime} \times \iota \gamma^{\prime} \phi . \quad \rho v a^{\prime} \cdot \gamma \rho . \iota \gamma^{\prime}$.

\section{7}

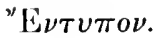




\section{8}

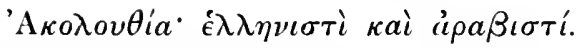

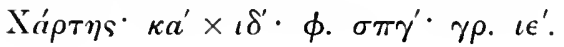

\section{9}

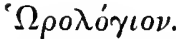

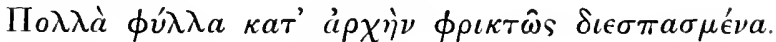

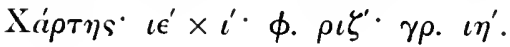

\section{0}

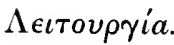

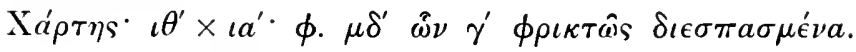

\section{1}

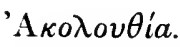

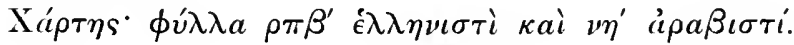

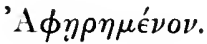

\section{2}

\section{3}

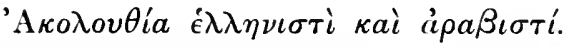

$\mathrm{X} a ́ \rho \tau \eta \varsigma^{\cdot} \iota \beta^{\prime} \times \theta^{\prime} \cdot \phi . \rho \xi \gamma^{\prime} \gamma \rho . \iota \beta^{\prime}$.

\section{4}

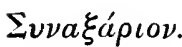

$\mathrm{X} \alpha \dot{\rho} \tau \eta \varsigma^{\cdot} \kappa \delta^{\prime} \times \iota \varsigma^{\prime} \phi . \sigma \eta^{\prime} \gamma \rho . \iota \zeta^{\prime}$. M. X. $a \phi o \delta^{\prime}$.

\section{5}

$\mathrm{T} a ́ \xi \iota s \tau \hat{\omega} \nu{ }^{\prime} \mathrm{E} \kappa \kappa \lambda \eta \sigma \iota \hat{\omega} \nu$.

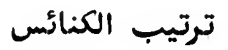

$\mathrm{X} \dot{\alpha} \rho \tau \eta \varsigma^{\cdot} \iota \zeta^{\prime} \times \iota \beta^{\prime} \cdot \phi . \rho \lambda \eta^{\prime} \gamma \rho . \iota \epsilon^{\prime}$.

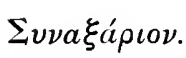

\section{6}

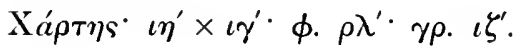




\section{7}

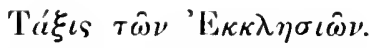

$$
\text { ترتيب كنائس ونظام }
$$

Х

\section{8}

مصحف ديونوسيوس تلهيذ الرسول بولص قاضى اساقفة الاثينا

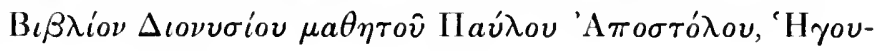

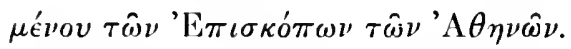

$\mathrm{X} \iota \rho \tau \eta \varsigma^{\cdot} \kappa \epsilon^{\prime} \times \iota \zeta^{\prime} \cdot \phi . \tau \iota \theta^{\prime} \cdot \gamma \rho . \iota \epsilon^{\prime}$.

\section{9}

كتاب القديس الفاضل اقليهس تلمهيذ السليح سمعان بطوس

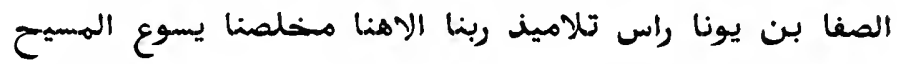

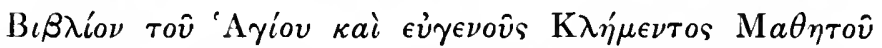

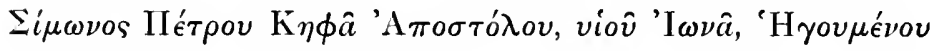

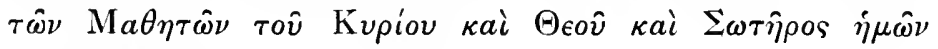
'I $\eta \sigma o \hat{v} \mathrm{X} \rho \iota \sigma \tau o \hat{v}$.

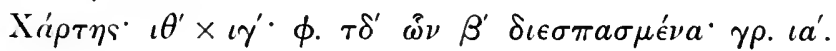

\section{0}

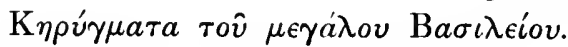

مقالات باسيليوس الكبير

$\mathrm{X} a ́ \rho \tau \eta \varsigma^{*} \kappa^{\prime} \times \iota \epsilon^{\prime} \cdot \phi . v \pi a^{\prime} \cdot \gamma \rho . \iota \delta^{\prime}$.

\section{1}

\section{لابينا المعظم في القديسين باسيليوس ريس اساقغة قيسارية}

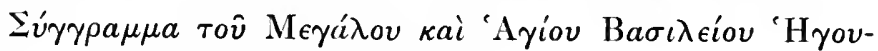

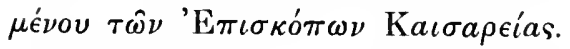

Х 


\section{2}

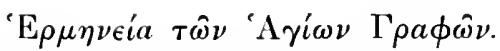

$$
\text { تغاسير }
$$

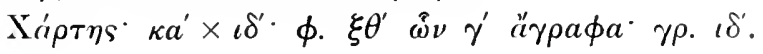

\section{3}

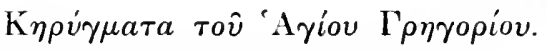

$$
\text { مياهر القديس اغريغوريوس }
$$

$\mathrm{X} \alpha \rho \tau \eta \varsigma^{*} \kappa \varsigma^{\prime} \times \iota \zeta^{\prime} \phi \phi . \phi \iota^{\prime} \gamma \rho . \iota \zeta^{\prime}$.

\section{4}

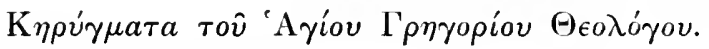

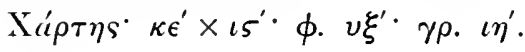

\section{5}

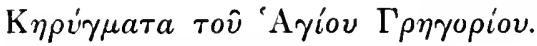

$$
\text { مقالات القديس اغريغوريوس }
$$

$\mathrm{X} \iota \rho \tau \eta \varsigma^{*} \kappa \delta^{\prime} \times \iota \zeta^{\prime} \phi . \tau \mu \delta^{\prime} \cdot \gamma \rho . \iota \zeta^{\prime}$.

\section{6}

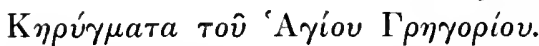

$$
\text { مقالات القديس اغريغوريوس }
$$

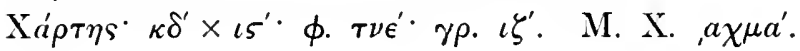

\section{7}

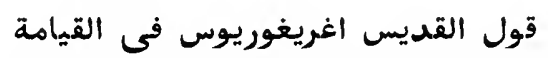

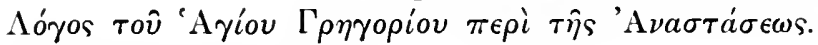

$\mathrm{M} \epsilon \mu \beta \rho a ́ \nu \eta^{\cdot} \iota \varsigma^{\prime} \times \iota \gamma^{\prime} \phi . \pi^{\prime} \gamma \rho . \iota \beta^{\prime}$.

\section{8}

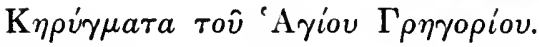

$$
\text { مقالات القديس اغريغوريوس }
$$

$\mathrm{X} a \dot{\rho} \tau \eta \varsigma^{\cdot} \kappa^{\prime} \times \iota \gamma^{\prime} \phi . \sigma \varphi \delta^{\prime} \gamma \rho . \imath \varsigma^{\prime}$.

G. A. M. 


\section{9}

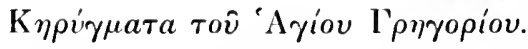

$$
\text { مقالات القديس اغريغوريوس }
$$

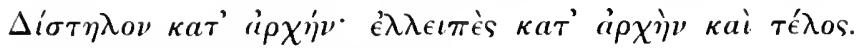

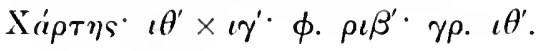

\section{0}

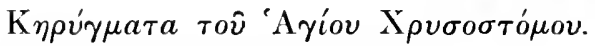

$$
\text { همالات القديس فم الفهب }
$$

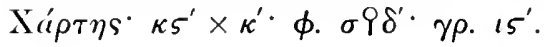

\section{1}

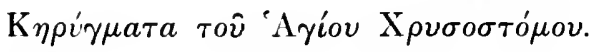

$$
\text { مقالات القديس فم الذهب }
$$

$\mathrm{X} a \rho \tau \eta \varsigma^{*} \kappa \varsigma^{\prime} \times \iota \zeta^{\prime} \phi . \tau \pi \zeta^{\prime} \gamma \rho . \imath \eta^{\prime}$.

\section{2}

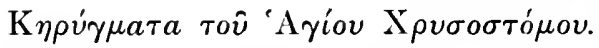

مقالات القديس فم الذهب

$\mathrm{X} \alpha \dot{\rho} \rho \eta \varsigma^{\cdot} \kappa \varsigma^{\prime} \times \imath \theta^{\prime} \cdot \phi . \sigma \iota^{\prime} \gamma \rho \cdot \kappa \varsigma^{\prime}$.

\section{3}

$\mathrm{K} \eta \rho \dot{\gamma} \mu a \tau a \xi \zeta^{\prime}(\dot{\alpha} \gamma \nu \omega \sigma \tau o v)$.

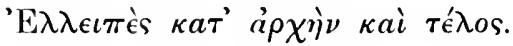

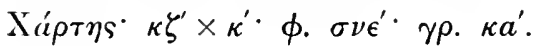

\section{4}

$$
\text { مقالات يوحنا فم الذهب }
$$

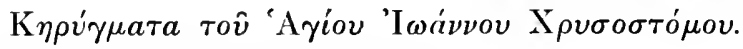

$\mathrm{X} a \dot{\rho} \tau \eta \varsigma^{\cdot}, \zeta^{\prime} \times \iota \gamma^{\prime} \phi . \sigma o \delta^{\prime} \cdot \gamma \rho . \iota \delta^{\prime}$. 


\section{5}

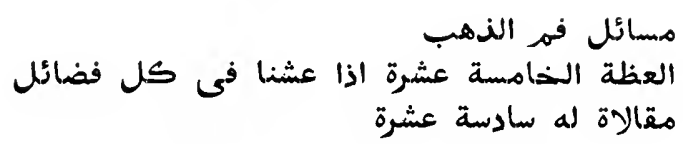

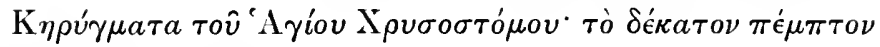

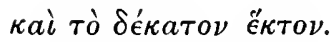

$\mathrm{X} \alpha \rho \tau \eta \varsigma^{\cdot} \kappa \beta^{\prime} \times \iota 5^{\prime} \phi . \quad \tau \lambda \beta^{\prime} \gamma \rho . \zeta^{\prime}$. M. X.,$a \sigma^{\prime}$.

\section{6}

$\mathrm{K} \eta \rho \dot{\gamma} \gamma \mu a \tau a \lambda \varsigma^{\prime}(\dot{a} \gamma \nu \omega \dot{\sigma} \sigma \tau o v)$.

re

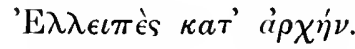

$\mathrm{X} \dot{\alpha} \rho \tau \eta \varsigma^{\cdot} \kappa \epsilon^{\prime} \times \iota \eta^{\prime} \phi . \tau \nu \epsilon^{\prime} \cdot \gamma \rho . \iota \theta^{\prime}$.

\section{7}

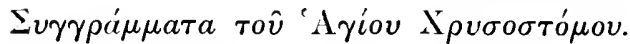

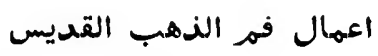

$\mathrm{X} a ́ \rho \tau \eta s^{\circ} \kappa \epsilon^{\prime} \times \iota \zeta^{\prime} \cdot \phi \cdot \rho \pi \theta^{\prime} \cdot \gamma \rho . \iota 5^{\prime}$.

\section{8}

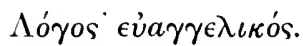

$\mathrm{X} i \rho \tau \eta \varsigma^{\cdot} \kappa \varsigma^{\prime} \times \kappa^{\prime} \phi \phi . \rho \nu \gamma^{\prime} \gamma \rho . \iota \eta^{\prime}$. M. X.,$a \sigma \iota \gamma^{\prime}$.

\section{9}

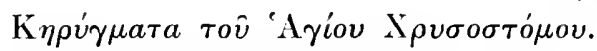

$$
\text { مقالات فم الذهب }
$$

$\mathrm{X} \alpha \dot{\alpha} \rho \eta \varsigma^{\cdot} \kappa \delta^{\prime} \times \iota \epsilon^{\prime} \cdot \phi . \phi \iota \theta^{\prime} \cdot \gamma \rho . \iota \eta^{\prime}$.

\section{0}

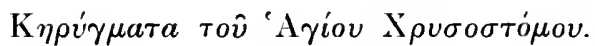

$$
\text { مقاللات فم الذهب }
$$

Х 


\section{1}

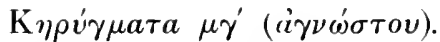

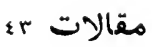

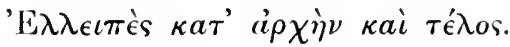

$\mathrm{X} a ́ \rho \tau \eta s^{\cdot} \kappa \epsilon^{\prime} \times \iota \varsigma^{\prime} \cdot \phi . v \epsilon^{\prime} \cdot \gamma \rho . \iota \zeta^{\prime}$.

\section{2}

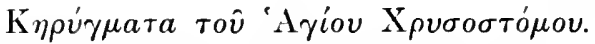

$$
\text { مقالات فم الذهب }
$$

$\mathrm{X} \dot{\rho} \rho \tau \eta \varsigma^{\prime} \kappa \epsilon^{\prime} \times 1 \varsigma^{\prime} \cdot \phi . \tau о \zeta^{\prime} \cdot \gamma \rho . \iota \zeta^{\prime}$.

\section{3}

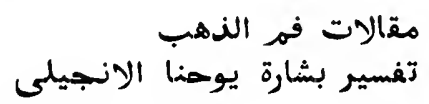

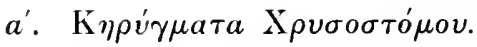

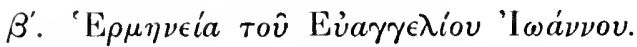

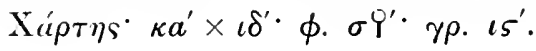

\section{4}

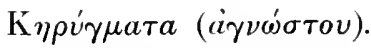

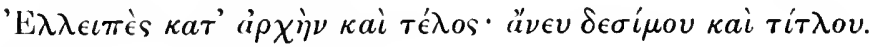

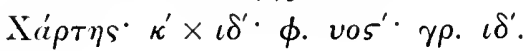

\section{5}

ذ̇vakípiov.

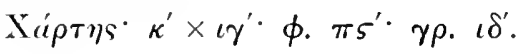

\section{6}

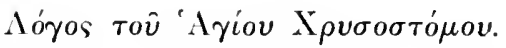

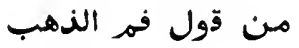

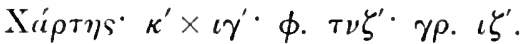




\section{7}

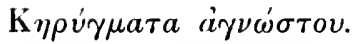

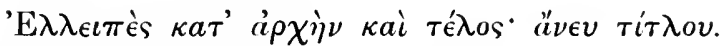

$\mathrm{X} a ́ \rho \tau \eta s^{\cdot} \iota \zeta^{\prime} \times \iota \gamma^{\prime} \phi . \sigma \nu \epsilon^{\prime} \gamma \rho . \iota \delta^{\prime}$.

\section{8}

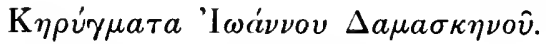

مقالات يوحنا الدمشقى

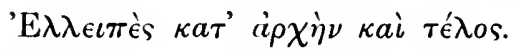

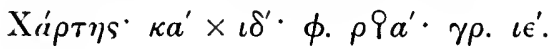

\section{9}

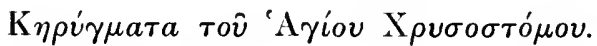

مقالات فم الذهب

$\mathrm{X} a \rho \tau \eta \varsigma^{\cdot} \kappa \beta^{\prime} \times \iota \delta^{\prime} \cdot \phi . \tau \iota a^{\prime} \gamma \rho . \iota \zeta^{\prime}$.

\section{0}

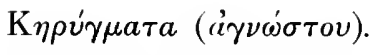

مقالت

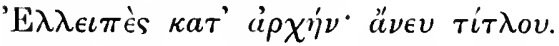

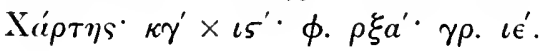

\section{1}

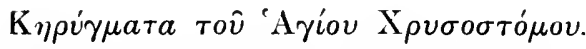

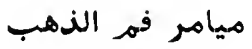

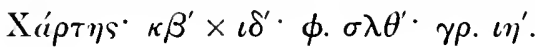

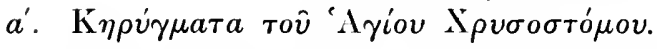

\section{2}

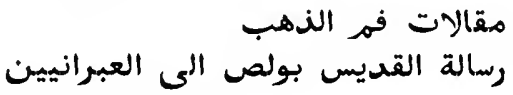

$\beta^{\prime}$. 'E

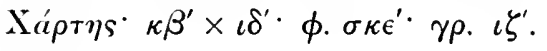




\section{3}

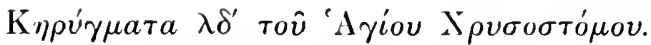

$$
\text { مقالات فم الذهب عا }
$$

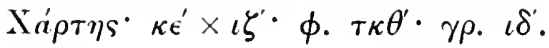

\section{4}

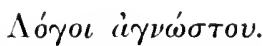

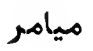

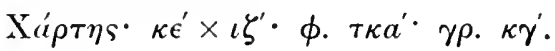

\section{5}

$$
\text { مقالات فم الذهب }
$$

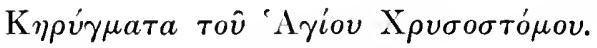

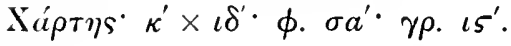

\section{6}

$$
\text { مقالات يوحنا الدمشقى فم الذهب والخ }
$$

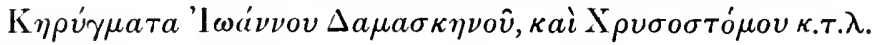

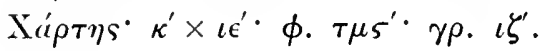

\section{7}

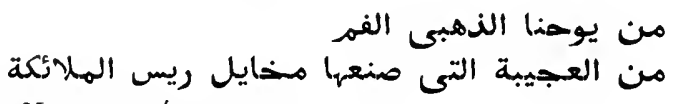

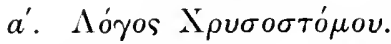

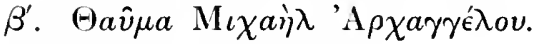

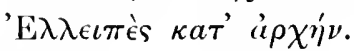

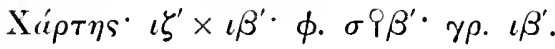

\section{8}

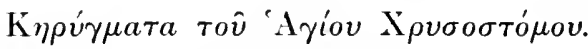

$$
\text { مقالע فم النهمب }
$$

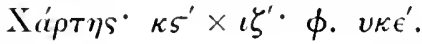

\section{9}

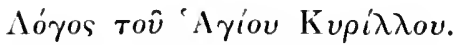

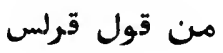

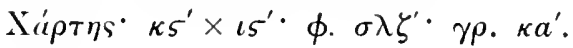




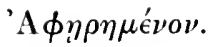

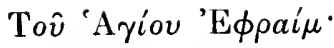

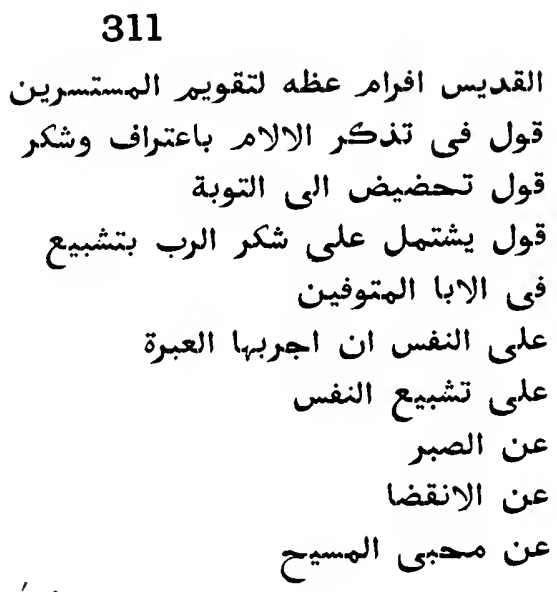

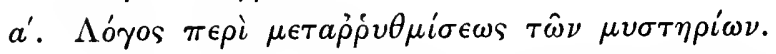

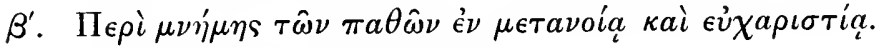

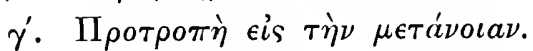

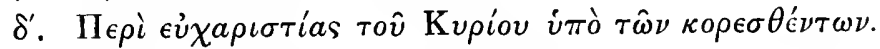

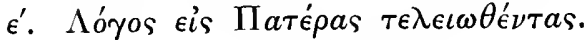

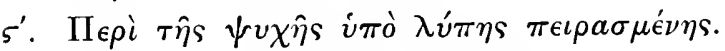

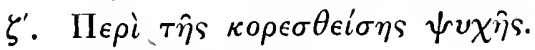

$\eta^{\prime}$. Пє

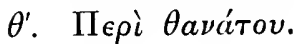

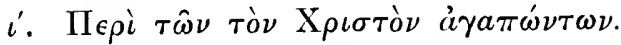

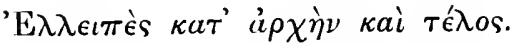

$M \epsilon \mu \beta \rho a ́ v \eta \eta^{\cdot} \kappa \varsigma^{\prime} \times \imath \eta^{\prime} \cdot \phi . \tau \xi \beta^{\prime} \cdot \gamma \rho . \iota \eta^{\prime}$.

\section{2}

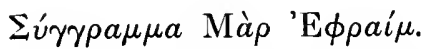

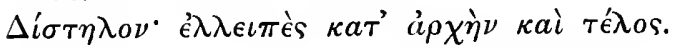

М $\epsilon \mu \beta \rho a^{\prime} \nu \eta^{\prime} \kappa \eta^{\prime} \times \kappa a^{\prime} \cdot \phi, \nu 5^{\prime} \cdot \gamma \rho . \kappa a^{\prime}$. 


\section{3}

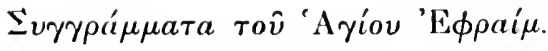

$$
\text { اعمال مار افرام }
$$

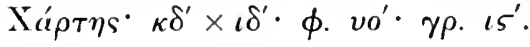

\section{4}

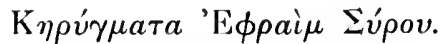

$$
\text { مقالעت مار افرام }
$$

'E $\mathrm{\lambda} \lambda \epsilon \iota \pi \hat{\epsilon} \varsigma \kappa a \tau$ '

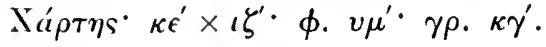

\section{5}

$$
\text { تعليم مار افراه السريانى }
$$

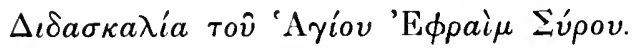

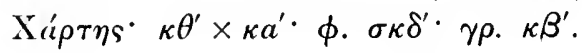

\section{6}

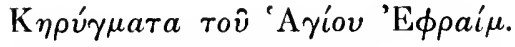

$$
\text { مقالات مار افرام }
$$

$\mathrm{X} \alpha \rho \tau \eta s^{\cdot} \iota \zeta^{\prime} \times \imath \gamma^{\prime} \phi . \sigma o \eta^{\prime} \gamma \rho . \kappa \gamma^{\prime}$.

\section{7}

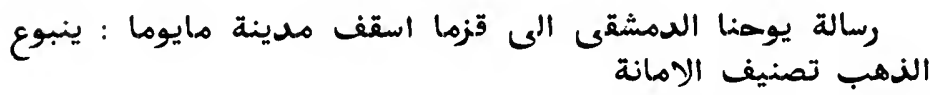

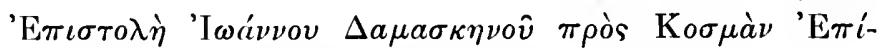

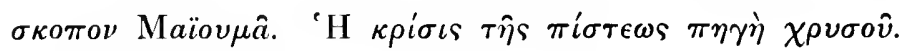

$\mathrm{X} a \dot{\rho} \tau \eta \varsigma^{\cdot} \kappa \delta^{\prime} \times \iota \zeta^{\prime} \cdot \phi . v \xi^{\prime} \gamma \rho . \iota \zeta^{\prime}$.

\section{8}

$a^{\prime} . \quad ' \mathrm{E} \pi \iota \sigma \tau o \lambda \eta{ }^{\prime} \mathrm{I} \omega \alpha \dot{\nu} \nu 0 v \Delta a \mu a \sigma \kappa \eta \nu o \hat{v}$.

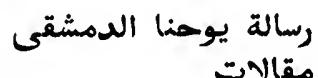

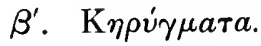

$\Delta \iota \epsilon \phi \theta a \rho \mu \epsilon^{\prime} \nu \nu \nu$ vंтò $\sigma \epsilon ́ \omega \nu$.

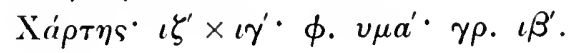




\section{9}

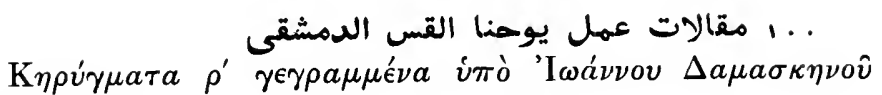

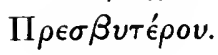

$\mathrm{X} a \dot{\rho} \tau \eta \varsigma^{\cdot} \kappa \beta^{\prime} \times \iota \delta^{\prime} \cdot \phi . \tau \nu \theta^{\prime} \gamma \rho . \iota \epsilon^{\prime}$.

\section{0}

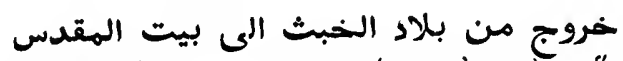

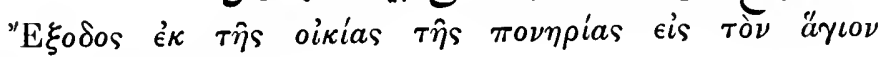

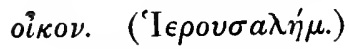

$\mathrm{X} a ́ \rho \tau \eta s^{\cdot} \iota \eta^{\prime} \times \iota \beta^{\prime} \cdot \phi . \sigma \iota \eta^{\prime} \gamma \rho . \iota \epsilon^{\prime}$.

\section{1}

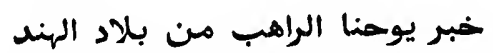

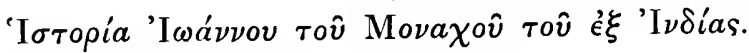

$\mathrm{X} a ́ \rho \tau \eta s^{\cdot} \iota \eta^{\prime} \times \iota \gamma^{\prime} \phi . \quad \sigma 9 \delta^{\prime} \cdot \gamma \rho . \iota \delta^{\prime}$.

\section{2}

\section{دخول من بلاد الخبث الى بيت الهقدس}

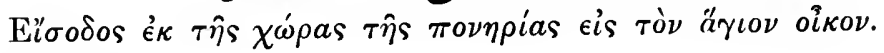
( $\mathrm{I} \mathrm{I} \epsilon \rho v \sigma a \lambda \eta \dot{\mu}$.)

$\mathrm{X} a ́ \rho \tau \eta s^{\cdot} \iota \eta^{\prime} \times \iota \delta^{\prime} \phi . \sigma \kappa \beta^{\prime} \gamma \rho . \iota \zeta^{\prime}$.

\section{3}

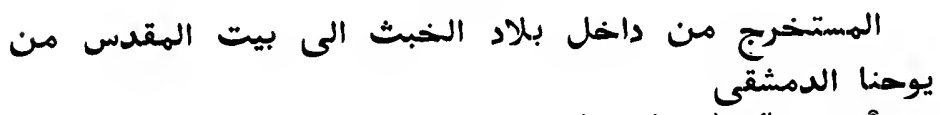

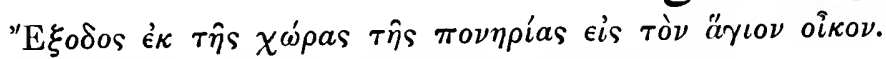

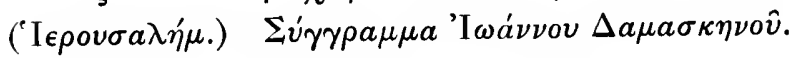

$\mathrm{X} a ́ \rho \tau \eta \varsigma^{\cdot} \iota \varsigma^{\prime} \times \iota a^{\prime} \cdot \phi . \tau \varsigma^{\prime} \cdot \gamma \rho . \iota \gamma^{\prime}$.

\section{4}

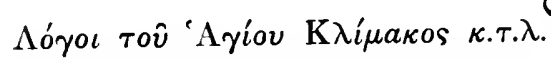

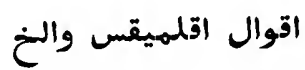

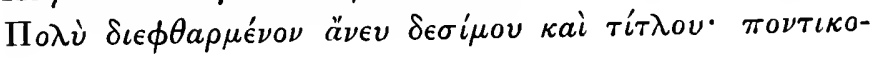
$\phi a ́ \gamma \eta \mu a$.

$\mathrm{X} a ́ \rho \tau \eta \varsigma^{\cdot} \kappa \beta^{\prime} \times \iota \epsilon^{\prime} \cdot \phi . \sigma \iota \epsilon^{\prime} \gamma \rho . \iota \eta^{\prime}$.

G. A. M. 


\section{5}

$$
\text { مواعظ القديس ثاوذوروس ريس دير الاسطق }
$$

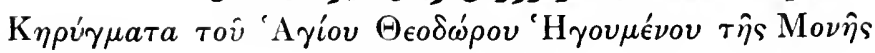

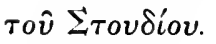

Хá $\rho \tau \eta s^{\cdot} \iota \epsilon^{\prime} \times \iota^{\prime} \phi . \sigma o a^{\prime} \cdot \gamma \rho . \iota \delta^{\prime}$.

\section{6}

$\Delta \iota \delta a \chi \grave{\eta}(\grave{a \gamma \nu \omega ́ \sigma \tau o v) . ~}$

$$
\text { تعليم }
$$

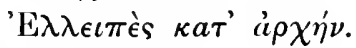

$\mathrm{X} a ́ \rho \tau \eta \varsigma^{\cdot} \kappa \delta^{\prime} \times \iota \zeta^{\prime} \cdot \phi . \sigma \iota \delta^{\prime} \cdot \gamma \rho . \iota a^{\prime}$.

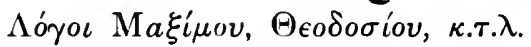

\section{7}

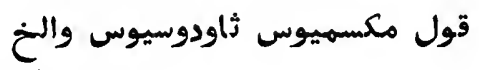

$\mathrm{X} \iota \rho \tau \eta \varsigma^{*} \kappa \delta^{\prime} \times \iota \epsilon^{\prime} \cdot \phi \cdot \rho o \delta^{\prime} \cdot \gamma \rho . \iota \zeta^{\prime}$.

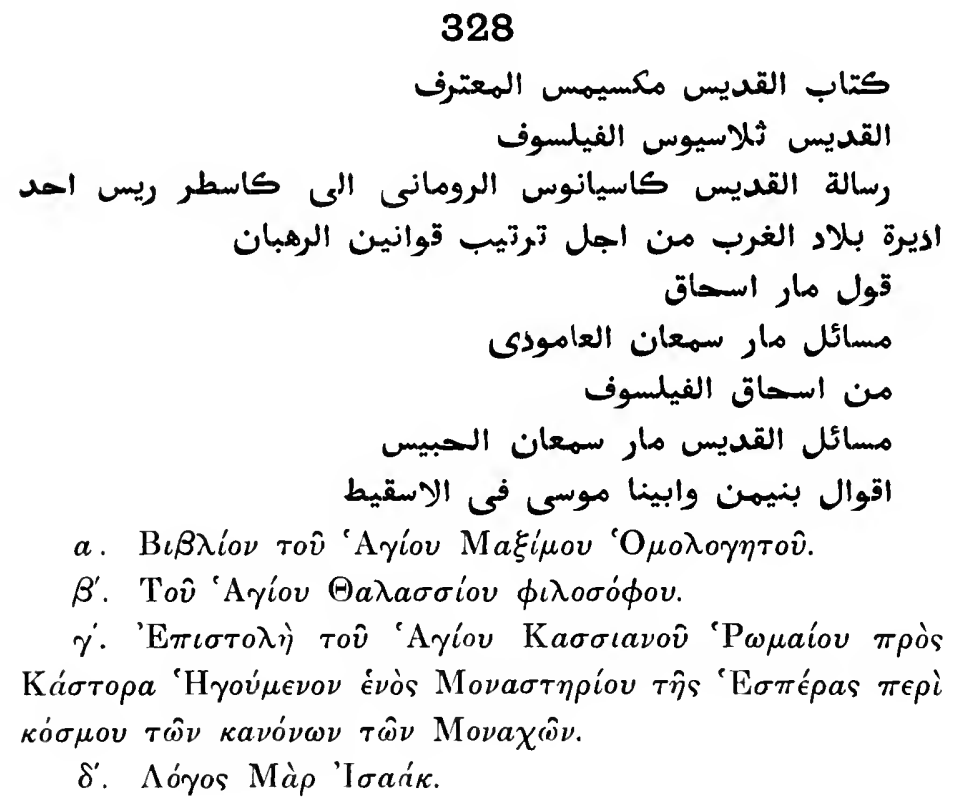




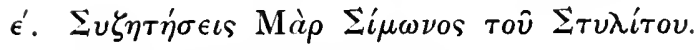

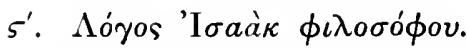

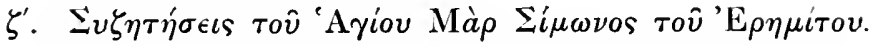

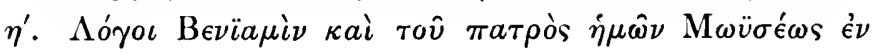
$\tau \hat{\eta} \Sigma_{\kappa} \hat{\eta} \tau \iota$.

$\mathrm{X} a \dot{\rho} \tau \eta \varsigma^{\cdot} \kappa^{\prime} \times \iota \epsilon^{\prime} \cdot \phi . \sigma \nu \zeta^{\prime} \omega^{\gamma} \nu \eta^{\prime} a^{\prime} \gamma \rho a \phi a^{\cdot} \gamma \rho . \iota \theta^{\prime}$.

\section{9}

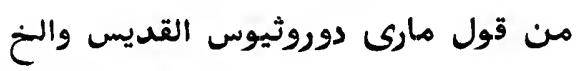

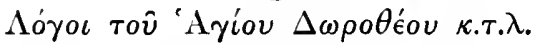

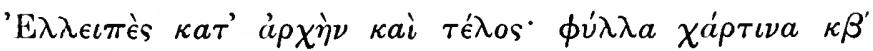
$\pi \rho{ }^{\prime} \iota \theta \dot{\varepsilon} \nu \tau a$.

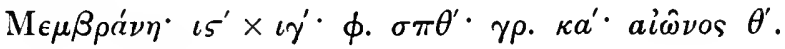

\section{0}

K $\eta \rho \dot{\gamma} \gamma \mu a \tau a(\dot{a} \gamma \nu \omega \dot{\sigma} \sigma o v)$.

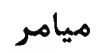

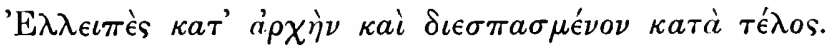

$\mathrm{X} a ́ \rho \tau \eta \varsigma^{\cdot} \iota \varsigma^{\prime} \times \iota \gamma^{\prime} \phi . \tau \pi \delta^{\prime} \cdot \gamma \rho . \iota \eta^{\prime}$.

\section{1}

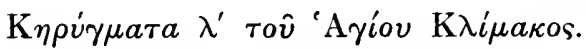

$$
\text { r. مقالات اقليمقس . }
$$

Ха́ $\rho \tau \eta \varsigma^{\cdot} \kappa \delta^{\prime} \times \iota \varsigma^{\prime} \cdot \phi . \sigma \iota^{\prime} \cdot \gamma \rho . \iota \eta^{\prime}$.

\section{2}

$\mathrm{K} \eta \rho \dot{\gamma} \gamma \mu a \tau a(\dot{a} \gamma \nu \omega \dot{\sigma} \sigma \tau o v)$.

'E $\mathrm{\lambda} \lambda \epsilon \iota \pi \dot{\epsilon} \varsigma \kappa a \tau^{\prime} a \rho \chi \eta \dot{\eta} \nu$.

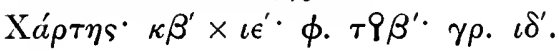

\section{3}

$\mathrm{K} \eta \rho \dot{\gamma} \gamma \mu a \tau a(a ̉ \gamma \nu \omega ́ \sigma \tau o u)$.

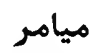

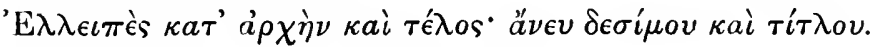

$\mathrm{X} a ́ \rho \tau \eta s^{\prime} \kappa a^{\prime} \times \iota \delta^{\prime} \cdot \phi . \rho \xi \theta^{\prime} \cdot \gamma \rho . \iota \eta^{\prime}$. 


\section{4}

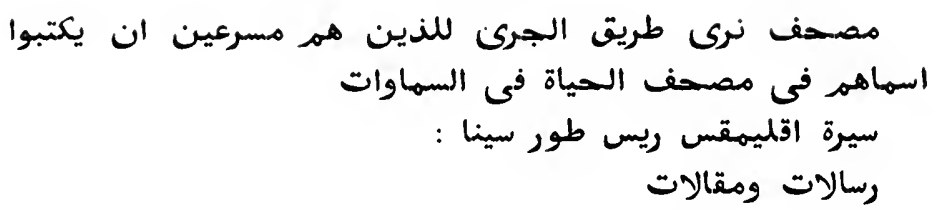

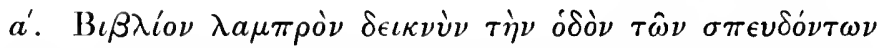

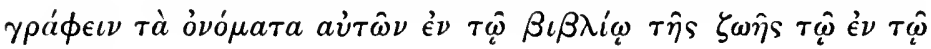
oúpavộ.

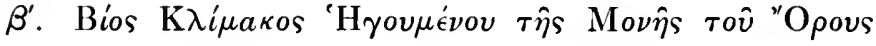
$\Sigma \iota v \hat{a}$.

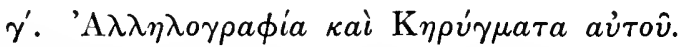

Х $а ́ \rho \tau \eta s^{\cdot} \kappa \theta^{\prime} \times \kappa a^{\prime} \cdot \phi . \rho \rho^{\prime} \cdot \gamma \rho . \quad \iota \eta^{\prime}$.

\section{5}

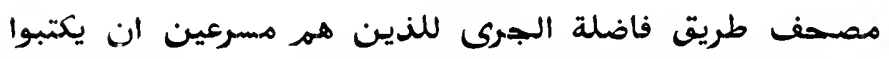
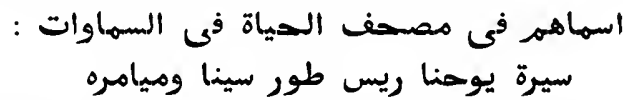

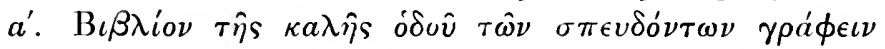

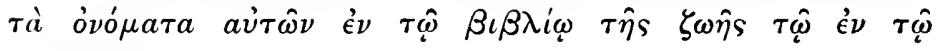

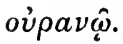

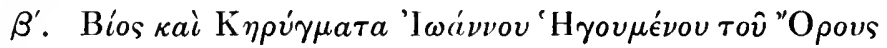
$\Sigma \iota v a \hat{a}$

$\mathrm{X} a ́ \rho \tau \eta \varsigma^{\cdot} \iota \eta^{\prime} \times \iota \gamma^{\prime} \phi . \sigma \kappa \varsigma^{\prime} \cdot \gamma \rho . \iota \epsilon^{\prime}$.

\section{6}

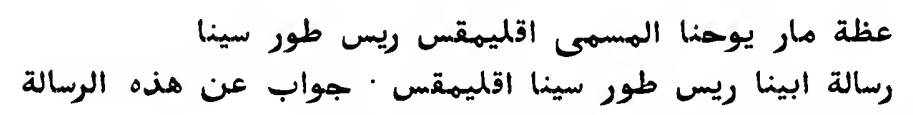

الهوتنف كتبها اليه ابينا يوحنا ريس رينا راية اليهميس

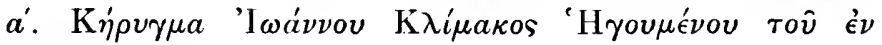

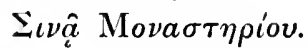

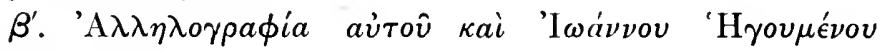

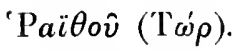

$\mathrm{X} a ́ \rho \tau \eta s^{\cdot} \kappa \epsilon^{\prime} \times 15^{\prime} \cdot \phi . \sigma \kappa 5^{\prime} \gamma \rho . \quad \zeta^{\prime}$. 


\section{7}

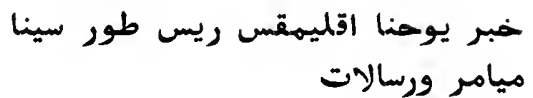

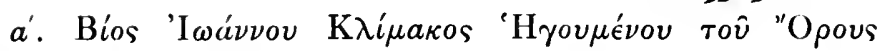
$\Sigma \iota v \hat{a}$.

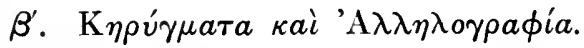

$\mathrm{X} a \rho \tau \eta \varsigma^{*} \kappa a^{\prime} \times \iota \epsilon^{\prime} \cdot \phi . \sigma \varsigma^{\prime} \cdot \gamma \rho . \iota \delta^{\prime}$.

\section{8}

مصحف طريق فاضلة الهجرى للذين هم مسرعين ان يكتبوا
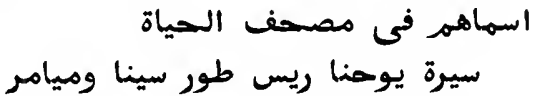

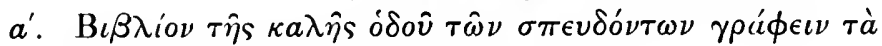

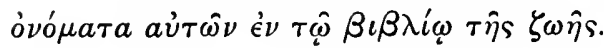

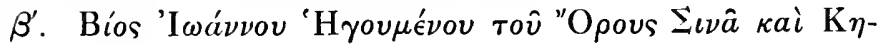

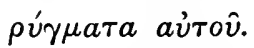

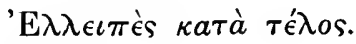

$\mathrm{X} \dot{\rho} \rho \tau \eta s^{\cdot} \kappa \varsigma^{\prime} \times \iota \zeta^{\prime} \phi . \rho \xi \beta^{\prime} \cdot \gamma \rho . \iota \theta^{\prime}$.

\section{9}

$$
\text { مقالات يوحنا ريس دير طور سينا }
$$

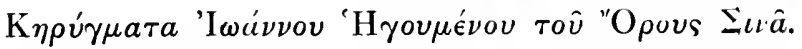
$\mathrm{X} a \rho \tau \eta \varsigma^{\circ} \kappa a^{\prime} \times \iota \epsilon^{\prime} \phi . \rho \theta^{\prime} \cdot \gamma \rho . \iota \epsilon^{\prime}$. M. X.,$a \psi \lambda \theta^{\prime}$.

\section{0}

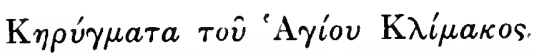

$$
\text { مقالات اقلميهقس }
$$

$\mathrm{X} а \dot{\rho} \tau \eta s^{\circ} \kappa \gamma^{\prime} \times \iota \varsigma^{\prime} \cdot \phi . \tau \kappa \epsilon^{\prime} \cdot \gamma \rho . \iota \gamma^{\prime}$.

\section{1}

خصة ثاوذرس وميامره

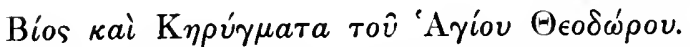

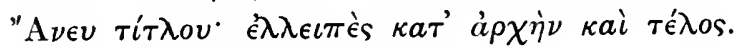

$\mathrm{X} a ́ \rho \tau \eta s^{\cdot} \kappa a^{\prime} \times \iota \delta^{\prime} \cdot \phi . \rho \nu \delta^{\prime} \cdot \gamma \rho . \iota \epsilon^{\prime}$. 


\section{2}

$$
\text { مقالات القديس يوحنا ريس طور سينا }
$$

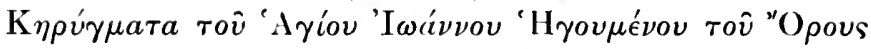
$\Sigma_{\iota \nu \hat{a}}$

$\mathrm{X} \alpha \dot{\rho} \tau \eta \varsigma^{\cdot} \kappa a^{\prime} \times \iota \varsigma^{\prime} \cdot \phi . \rho \mu \gamma^{\prime} \gamma \rho . \kappa a^{\prime}$.

\section{3}

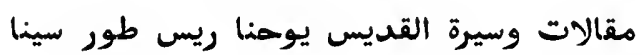

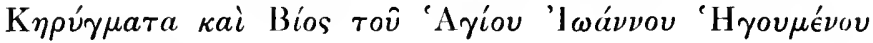

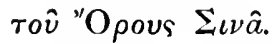

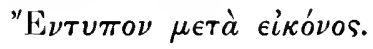

\section{4}

رسالات اقلميهقس ويوحنا ريس دير راية

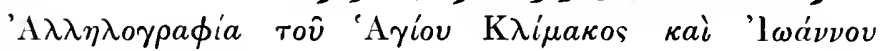

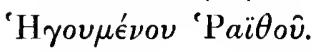

$\mathrm{X} a ́ \rho \tau \eta \varsigma^{\cdot} \kappa \varsigma^{\prime} \times \iota \eta^{\prime} \cdot \phi . \sigma \iota \varsigma^{\prime} \cdot \gamma \rho . \iota \zeta^{\prime}$.

\section{5}

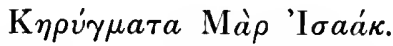

$$
\text { مقالات مار اسحاق }
$$

Ха́ $\rho \tau \eta \varsigma^{\cdot} \kappa \varsigma^{\prime} \times \iota \zeta^{\prime} \cdot \phi . \sigma \nu \gamma^{\prime} \cdot \gamma \rho . \iota \zeta^{\prime}$.

\section{6}

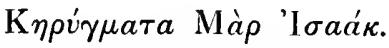

$$
\text { مقالات مار اسحاق }
$$

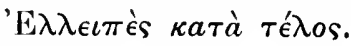

$\mathrm{X} a^{\prime} \rho \tau \eta \varsigma^{\cdot} \kappa \gamma^{\prime} \times \iota 5^{\prime} \cdot \phi . \tau \kappa a^{\prime} \cdot \gamma \rho . \iota \varsigma^{\prime}$.

\section{7}

مقالات مار اسحاق وهار افرام

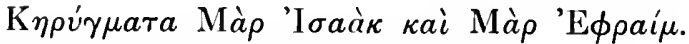

$\mathrm{X} \dot{a} \rho \tau \eta \varsigma^{\cdot} \kappa \epsilon^{\prime} \times \iota \zeta^{\prime} \cdot \phi . \sigma \gamma^{\prime} \cdot \gamma \rho . \iota \zeta^{\prime}$. 


\section{8}

ميامر · مصحف طريق فاضلة الهمبرى للنين هم مسرعين

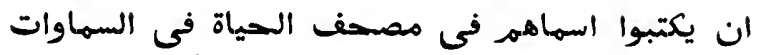

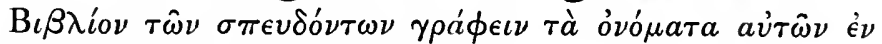

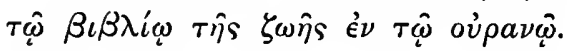

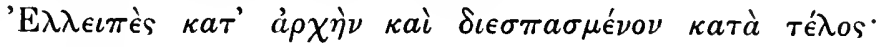

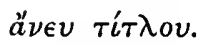

$\mathrm{X} a ́ \rho \tau \eta \varsigma^{\cdot} \kappa^{\prime} \times \iota \gamma^{\prime} \cdot \phi . \sigma o \gamma^{\prime} \cdot \gamma \rho . \iota \theta^{\prime}$.

\section{9}

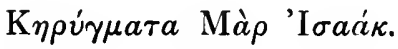

$$
\text { مقالات مار اسحاق }
$$

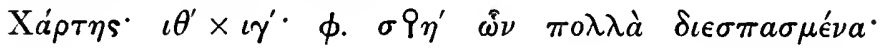
$\gamma \rho . \iota \epsilon^{\prime}$.

\section{0}

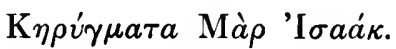

$$
\text { مقالات مار اسحاق }
$$

$\mathrm{X} a ́ \rho \tau \eta s^{\cdot} \iota \zeta^{\prime} \times \iota \gamma^{\prime} \cdot \phi \cdot \tau \beta^{\prime} \cdot \gamma \rho . \iota \gamma^{\prime}$.

\section{1}

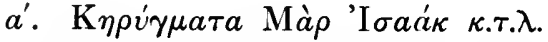

$$
\text { توارينخ ملوك اسرايل مار والخ }
$$

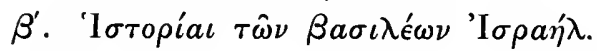

$\mathrm{X} a \dot{\rho} \tau \eta \varsigma^{\cdot} \kappa a^{\prime} \times \iota \epsilon^{\prime} \cdot \phi . \tau \xi \zeta^{\prime} \cdot \gamma \rho . \iota \theta^{\prime} \cdot \iota a^{\prime} \cdot \iota \delta^{\prime}$.

\section{2}

$$
\text { مقالات مائل واجوبة اسحاق الهطران }
$$

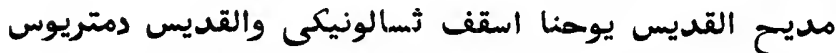

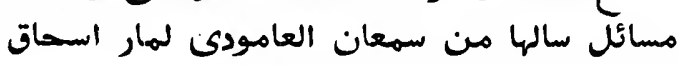

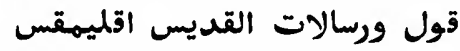

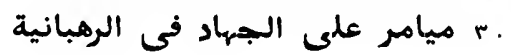


$a^{\prime} . \Sigma v \zeta \eta \tau \eta \dot{\sigma} \sigma \iota \iota$.

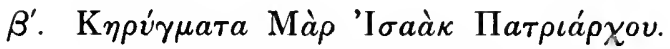

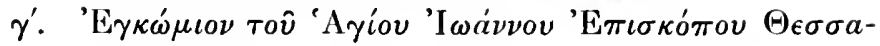

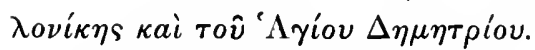

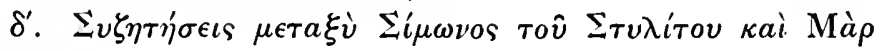
'Iбaáк.

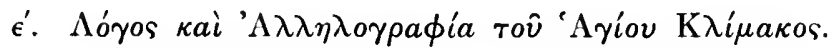

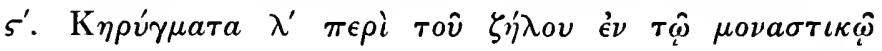
$\beta i \omega$.

$\mathrm{X} a ́ \rho \tau \eta \varsigma^{\cdot} \kappa \delta^{\prime} \times \iota 5^{\prime} \cdot \phi . \tau \lambda \delta^{\prime} \cdot \gamma \rho . \iota \theta^{\prime}$.

\section{3}

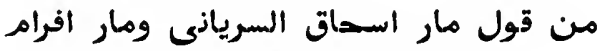

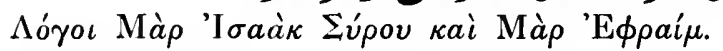

$\mathrm{X} a \rho \tau \eta s^{\cdot} \iota \zeta^{\prime} \times \iota \gamma^{\prime} \phi . v \imath \delta^{\prime} \cdot \gamma \rho . \iota \gamma^{\prime}$.

\section{4}

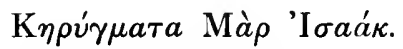

$$
\text { مقالات مار اسحاق }
$$

$\Pi o \lambda \dot{v} \delta \iota \epsilon \phi \theta a \rho \mu \epsilon \dot{\nu o \nu .}$

$\mathrm{X} a ́ \rho \tau \eta \varsigma^{\cdot} \iota \theta^{\prime} \times \iota \gamma^{\prime} \phi . \rho \pi \beta^{\prime} \cdot \gamma \rho . \kappa^{\prime}$.

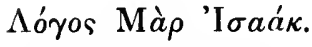

\section{5}

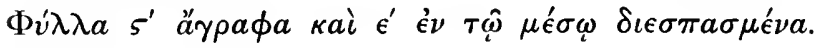

$\mathrm{X} a ́ \rho \tau \eta \varsigma^{*} \phi . \tau \lambda \epsilon^{\prime}$.

\section{6}

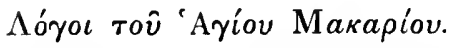

$$
\text { عظات القديس مقاريوس }
$$

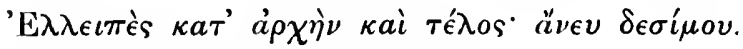

$\mathrm{X} a ́ \rho \tau \eta \varsigma^{\cdot} \kappa \epsilon^{\prime} \times \iota \zeta^{\prime} \phi . \sigma \iota \epsilon^{\prime} \gamma \rho . \iota \theta^{\prime}$. 


\section{7}

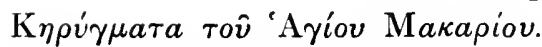

ميأمر القديس مقاريوس

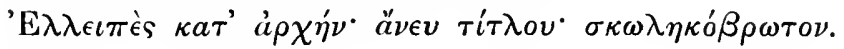

$\mathrm{X} a ́ \rho \tau \eta \varsigma^{*} \iota \theta^{\prime} \times \iota \delta^{\prime} \cdot \phi . \tau \xi \eta^{\prime} \cdot \gamma \rho . \kappa \varsigma^{\prime}$.

\section{8}

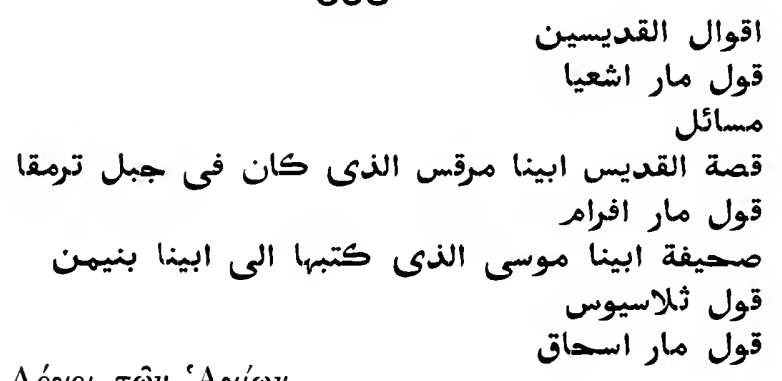

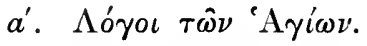

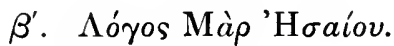

$\gamma^{\prime} . \Sigma v \zeta \eta \tau \dot{\eta} \sigma \epsilon \iota s$.

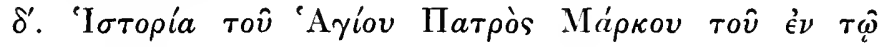
ö $\rho \iota \iota \Theta а \rho \mu а \kappa \hat{~}$

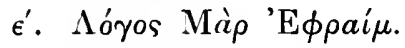

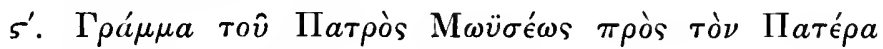

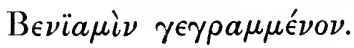

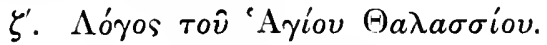

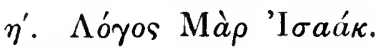

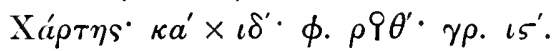

358 bis

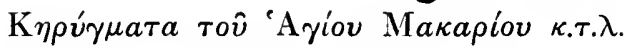

$$
\text { مي'مر القديس مقاريوس والخ }
$$

$\mathrm{X} a ́ \rho \tau \eta \varsigma^{\cdot} \iota \varsigma^{\prime} \times \iota \beta^{\prime} \cdot \phi . \sigma \pi \theta^{\prime} \cdot \gamma \rho . \iota \gamma^{\prime}$.

\section{9}

$$
\text { مسائل بين قديس وعرب فلان }
$$

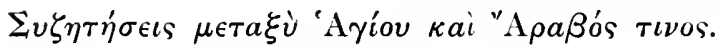

$\mathrm{X} a ́ \rho \tau \eta \varsigma^{\cdot} \kappa a^{\prime} \times \iota \delta^{\prime} \cdot \phi . \sigma \mu \epsilon^{\prime} \cdot \gamma \rho . \iota 5^{\prime}$.

G. A. M. 


\section{0}

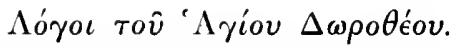

اقوال القديس ذراثيوس

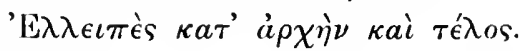

$\mathrm{X} \alpha \rho \rho \eta \varsigma^{\cdot} \kappa a^{\prime} \times \iota \epsilon^{\prime} \cdot \phi . \sigma \mu \epsilon^{\prime} \cdot \gamma \rho . \iota \eta^{\prime}$.

\section{1}

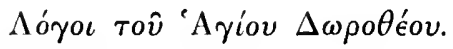
اقوال ذراثيوس

$\mathrm{X} \alpha \rho \tau \eta \varsigma^{\cdot} \kappa a^{\prime} \times \iota \delta^{\prime} \cdot \phi . \sigma \kappa \delta^{\prime} \cdot \gamma \rho, \iota \delta^{\prime}$.

362

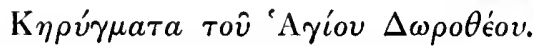

$$
\text { مقالات القديس ذوروثاوس }
$$

$\mathrm{X} a \dot{\rho} \rho \eta \varsigma^{\circ} \iota 5^{\prime} \times \imath \beta^{\prime} \cdot \phi . \sigma o \epsilon^{\prime} \cdot \gamma \rho . \iota \gamma^{\prime}$.

\section{3}

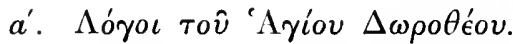

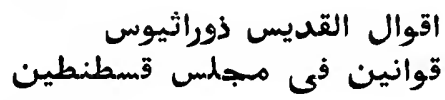

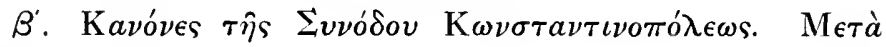
єiкóvos.

$\mathrm{X} \boldsymbol{\alpha}^{\prime} \rho \tau \eta \varsigma^{*} \iota \zeta^{\prime} \times \iota \beta^{\prime} \cdot \phi . \sigma \mu \epsilon^{\prime} \cdot \gamma \rho . \iota \epsilon^{\prime}$.

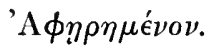

\section{4}

\section{5}

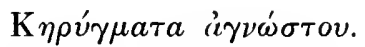

ميامر

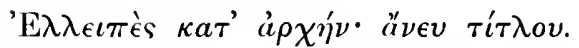

$\mathrm{X} \alpha \dot{\alpha} \rho \eta \varsigma^{\cdot} \kappa \beta^{\prime} \times \iota \delta^{\prime} \cdot \phi . \sigma \kappa \alpha^{\prime} \cdot \gamma \rho . \iota 5^{\prime}$.

\section{6}

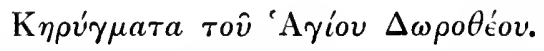
ميامر القديس ذروثاوس

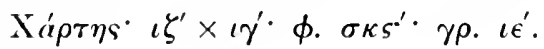




\section{7}

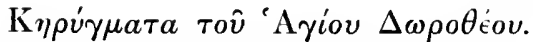

مقالات القديس ذروثاوس

Ха́ $\rho \tau \eta \varsigma^{\cdot} \kappa^{\prime} \times \iota \delta^{\prime} \cdot \phi . \rho o \epsilon^{\prime} \cdot \gamma \rho . \iota \zeta^{\prime}$.

\section{8}

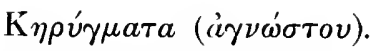

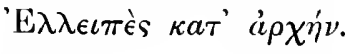

Х $a^{\prime} \rho \tau \eta \varsigma^{\cdot} \kappa a^{\prime} \times \iota \delta^{\prime} \cdot \phi . \sigma \pi \gamma^{\prime} \cdot \gamma \rho . \iota \eta^{\prime}$.

\section{9}

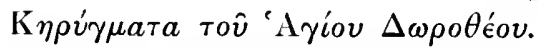

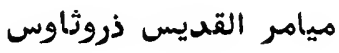

Ха́ $\rho \tau \eta \varsigma^{\cdot} \iota \eta^{\prime} \times \iota \beta^{\prime} \cdot \phi . \rho \gamma^{\prime} \gamma \rho . \iota \beta^{\prime}$.

\section{0}

a'. 'E

رسالة انتيخخس الراهب

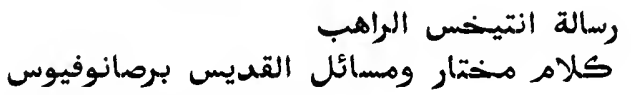

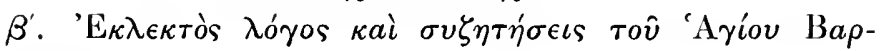

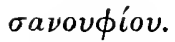

$\mathrm{X} a ́ \rho \tau \eta \varsigma^{\cdot} \kappa \delta^{\prime} \times \iota \epsilon^{\prime} \cdot \phi . \sigma \mu^{\prime} \gamma \rho . \kappa \gamma^{\prime}$.

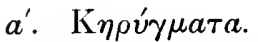

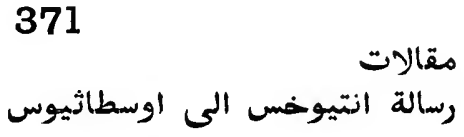

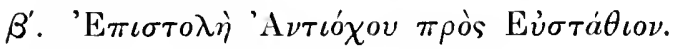

$\mathrm{X} \alpha \rho \tau \eta \varsigma^{\cdot} \kappa \delta^{\prime} \times \iota \zeta^{\prime} \phi . \rho \iota^{\prime} \gamma \rho . \iota s^{\prime}$.

\section{2}

$\mathrm{K} \eta \rho \dot{\gamma} \mu a \tau a$ à $\gamma \nu \omega \dot{\sigma \tau o v . ~}$

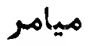

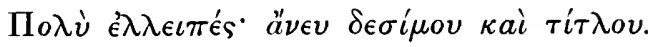

$\mathrm{X} \alpha \rho \tau \eta s^{*} \iota \eta^{\prime} \times \iota \gamma^{\prime} \cdot \phi . \sigma \mu a^{\prime} \cdot \gamma \rho . \iota \epsilon^{\prime}$. 


\section{3}

رسالة انتيوخس الراهب الى اوسطاثيوس ريس دير الاطالنيس من قول القديس ذوروثاوس التسو الفس

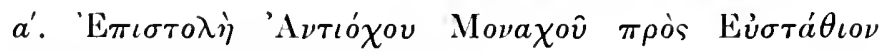
'H

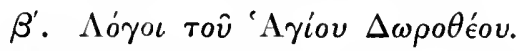

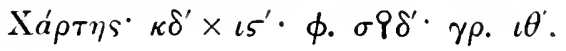

\section{4}

\section{رسالة انتيوخس الى اوسطأثيوس وميامره}

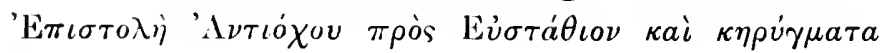
aข่งoิิ.

$\mathrm{X} a ́ \rho \tau \eta \varsigma^{\cdot} \kappa \epsilon^{\prime} \times \iota \zeta^{\prime} \cdot \phi . \rho \pi \epsilon^{\prime} \cdot \gamma \rho . \iota \theta^{\prime}$.

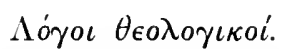

\section{5}

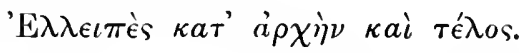

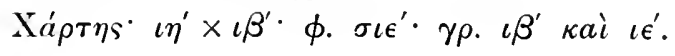

\section{6}

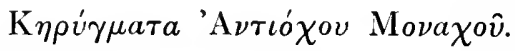

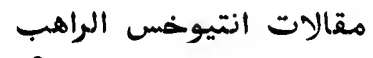

$\mathrm{X} \dot{\alpha} \rho \tau \eta \varsigma^{\cdot} \kappa \varsigma^{\prime} \times \imath \eta^{\prime} \cdot \phi . \sigma \nu^{\prime} \cdot \gamma \rho . \iota \zeta^{\prime}$.

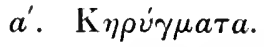

\section{7}

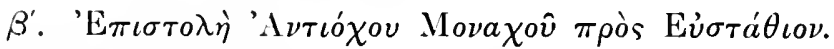

$\mathrm{X} \alpha \rho \tau \eta \varsigma^{\cdot} \kappa a^{\prime} \times \iota \delta^{\prime} \cdot \phi . \sigma \mu a^{\prime} \cdot \gamma \rho . \kappa a^{\prime}$.

\section{8}

$$
\text { رسالة انتيوخس الراهب الى اوسطاثيوس واقوال }
$$

'E

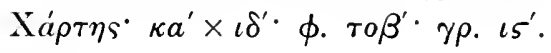




\section{9}

رسالة انتيوخس الراهب الذى من دير القديس مار سابا الى

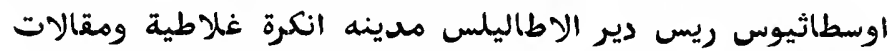

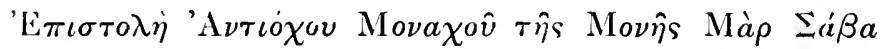

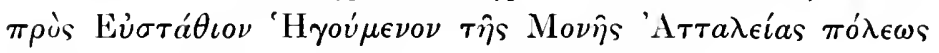

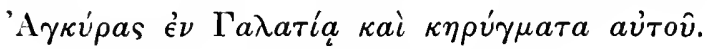

$\mathrm{X} a ́ \rho \tau \eta \varsigma^{*} \kappa a^{\prime} \times \iota \epsilon^{\prime} \cdot \phi . \sigma \iota \theta^{\prime} \cdot \gamma \rho$. $\iota \epsilon^{\prime}$.

\section{0}

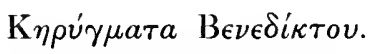

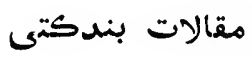

$\mathrm{X} a ́ \rho \tau \eta \varsigma^{\cdot} \kappa a^{\prime} \times \iota \delta^{\prime} \cdot \phi . \tau \varsigma^{\prime} \cdot \gamma \rho . \iota \epsilon^{\prime}$.

\section{1}

رسالة انتيوخس الراهب ومقالات

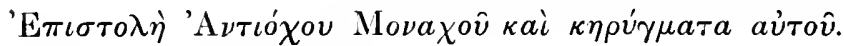

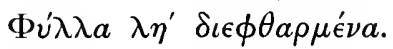

$\mathrm{X} a ́ \rho \tau \eta s^{\cdot} \kappa^{\prime} \times \iota \delta^{\prime} \cdot \phi . \sigma o \beta^{\prime} \cdot \gamma \rho . \iota \varsigma^{\prime}$.

\section{2}

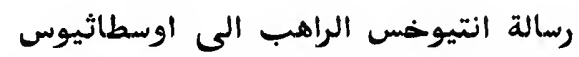

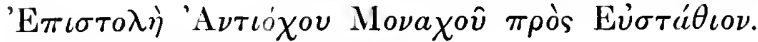

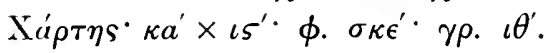

\section{3}

$\Sigma v \zeta \eta \tau \eta \dot{\sigma} \sigma \iota \varsigma$.

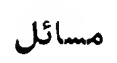

$\Phi \rho \iota \kappa \tau \hat{\omega} s \delta \iota \epsilon \phi \theta \rho \mu \epsilon ́ \nu o \nu$.

$\mathrm{X} a ́ \rho \tau \eta \varsigma^{\circ} \kappa^{\prime} \times \iota \delta^{\prime} \phi . \rho \epsilon^{\prime} \hat{\omega} \nu \eta^{\prime} a^{\prime} \gamma \rho a \phi a \cdot \gamma \rho . \iota \epsilon^{\circ}$.

\section{4}

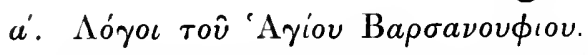

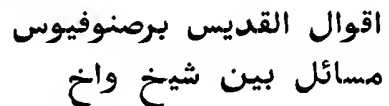

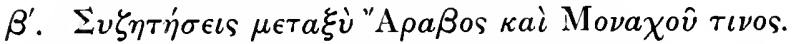

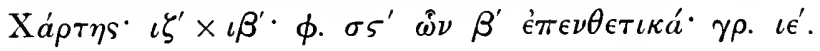




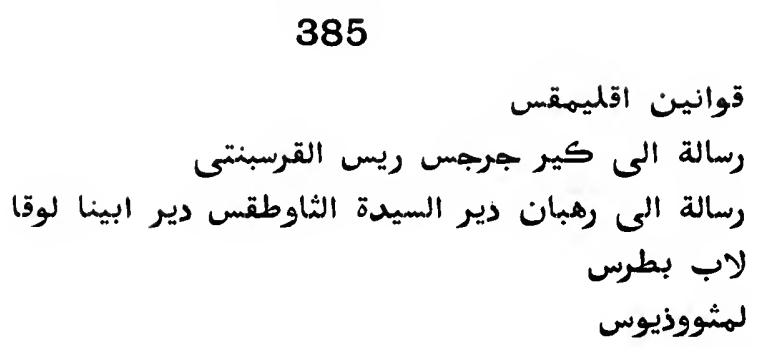

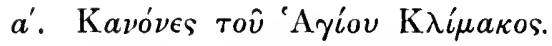

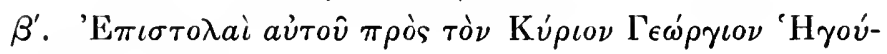
$\mu \in \nu o \nu$ (Кирцакой;).

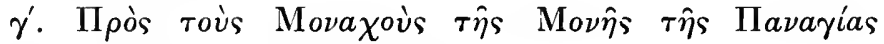

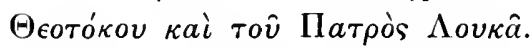

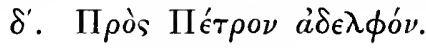

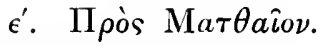

$\mathrm{X} \dot{\alpha} \rho \tau \eta \varsigma^{\cdot} \kappa \epsilon^{\prime} \times \iota \zeta^{\prime} \cdot \phi . v \nu^{\prime} \cdot \gamma \rho . \iota \eta^{\prime}$.

\section{6}

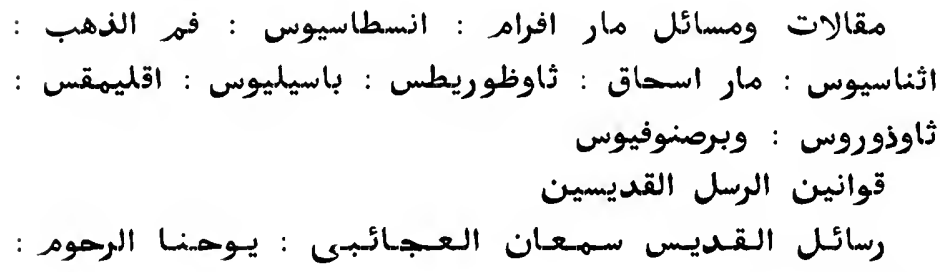

واغريغوريوس وساس

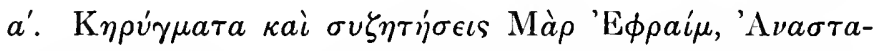

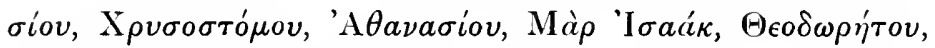

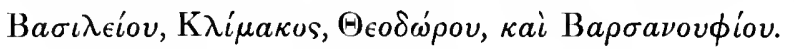

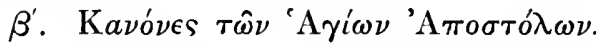

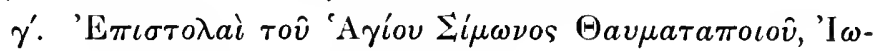

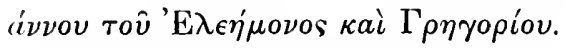

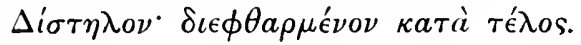

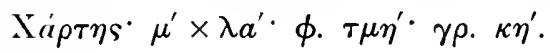




\section{7}

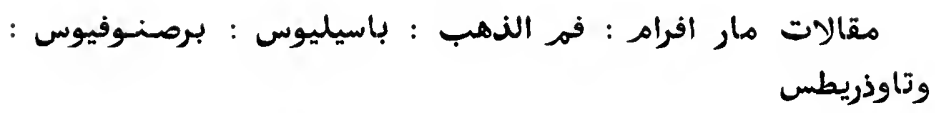

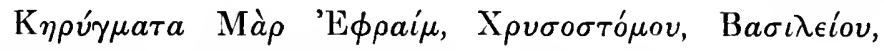

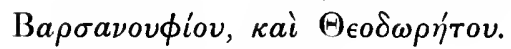

$\mathrm{X} a ́ \rho \tau \eta \varsigma^{*} \phi . \phi \lambda \beta^{\prime}$.

\section{8 ) \\ 389}

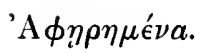

\section{0}

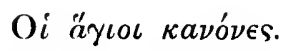

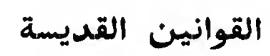

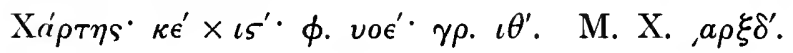

\section{1}

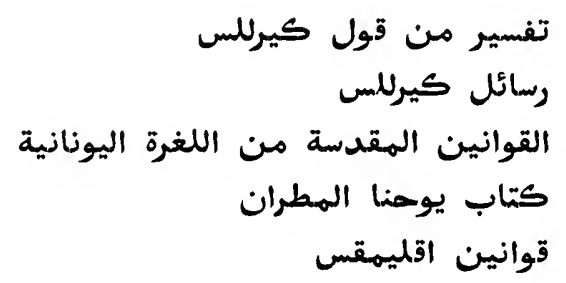

$a^{\prime}$. 'Е $\rho \mu \eta \nu \epsilon i ́ a \mathrm{~K} v \rho i ́ \lambda \lambda o v$.

$\beta^{\prime}$. 'E $\pi \iota \sigma \tau o \lambda a i \mathrm{~K} v \rho i \lambda \lambda o v$.

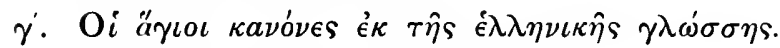

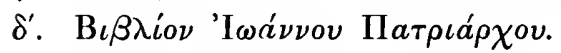

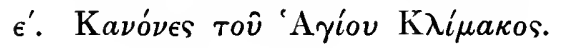

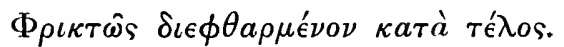

$\mathrm{X} a ́ \rho \tau \eta \varsigma^{*} \kappa \eta^{\prime} \times \kappa^{\prime} \cdot \phi . \sigma \pi \theta^{\prime}$. M. X.,$a \rho \xi \delta^{\prime}$. 


\section{2}

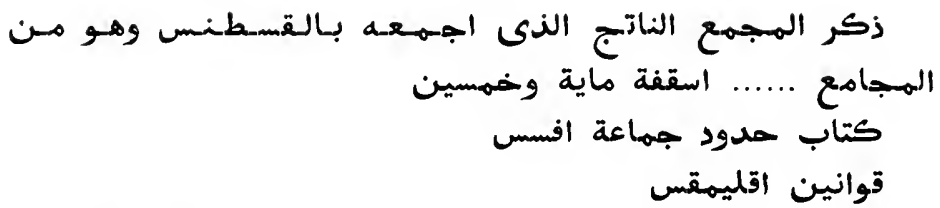

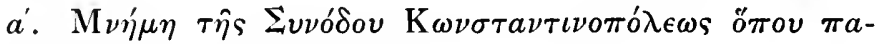
$\rho \hat{\eta} \sigma a \nu$ 'Е $\pi i \sigma \kappa o \pi \circ \iota \rho \nu^{\prime}$.

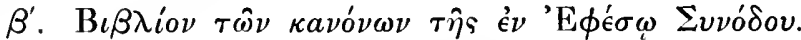

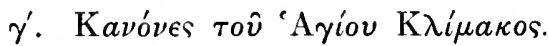

$\mathrm{X}^{\prime} \rho \tau \tau \varsigma^{\prime} \iota \varsigma^{\prime} \times \iota \beta^{\prime} \cdot \phi . \quad \sigma \xi \zeta^{\prime} \cdot \gamma \rho . \iota a^{\prime}$.

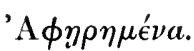

\section{$\left.\begin{array}{l}393 \\ 394\end{array}\right\}$}

\section{5}

قصة ثقلة القديسة : قصة اوفرسنية : قصة صافينيوس

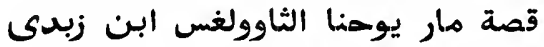

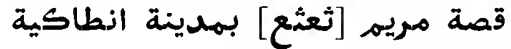

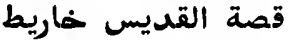
قصة مار كيراكس المس يس قصة القديس اغريغوريوس

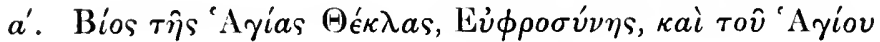
¿aßivov.

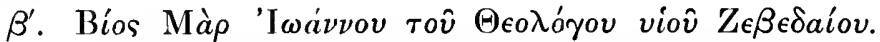

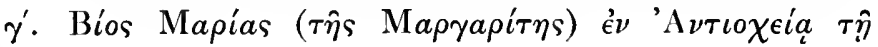
$\pi o ́ \lambda \epsilon \iota$.

$\delta^{\prime}$. Bíos tồ 'A yíov Xápıtos.

$\epsilon^{\prime}$. Bíos Mà Kuрıккоv.

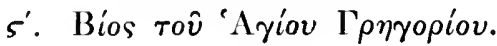

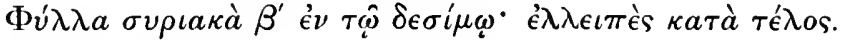

Хí $\rho \tau \eta \varsigma^{\cdot} \lambda a^{\prime} \times \kappa a^{\prime} \cdot \phi . \sigma \iota^{\prime} \cdot \gamma \rho . \kappa^{\prime}$. 


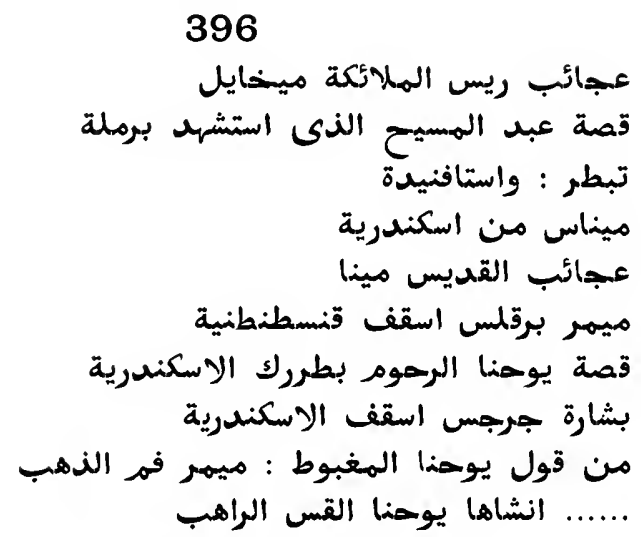

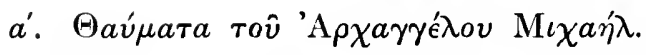

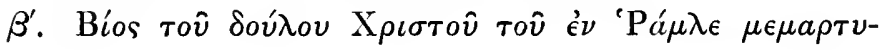

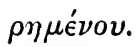

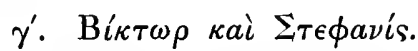

$\delta^{\prime} . \mathrm{M} \eta \nu \hat{a} \varsigma$ 'A $\mathrm{A} \epsilon \xi a \nu \delta \rho \epsilon i a \varsigma$.

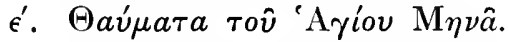

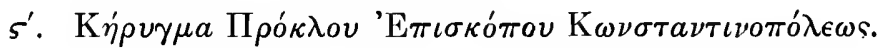

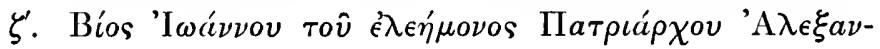
$\delta \rho \in i ́ a s$.

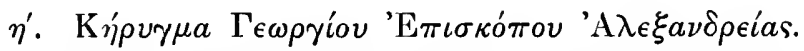

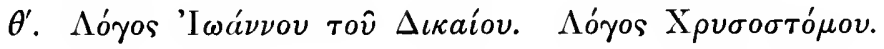

i. 'I

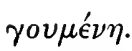

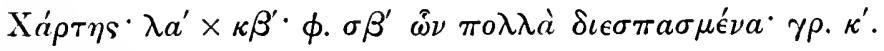
M. X.,$\iota \circ \theta^{\prime}$.

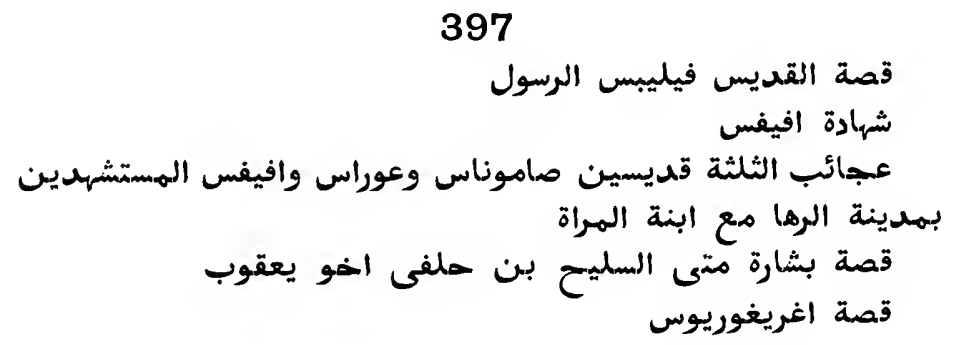

G. A. M. 


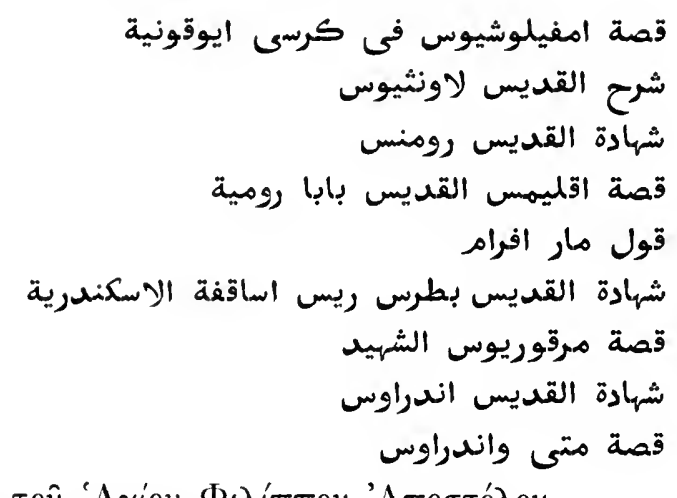

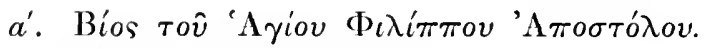

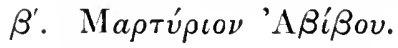

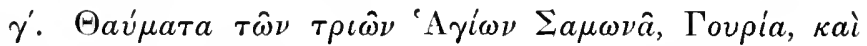

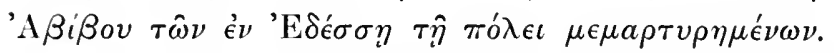

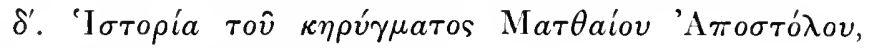

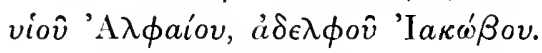

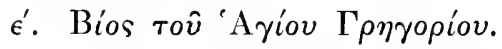

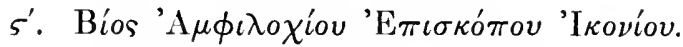

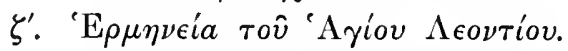

$\eta$ '. Bios tov 'A yiov 'P $\mathrm{\omega} \mu a \nu o \hat{v}$.

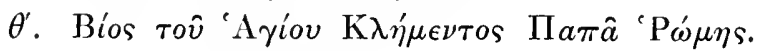

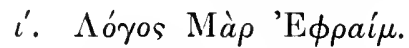

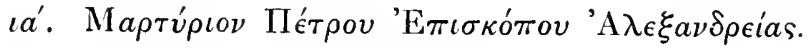

$\iota \beta^{\prime}$. Bíos Mepкovpíov Máprvpos.

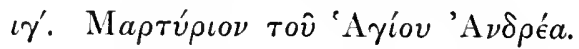

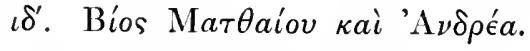

$\mathrm{X} \alpha \rho \tau \eta \varsigma^{*} \lambda a^{\prime} \times \kappa a^{\prime} \cdot \phi . \sigma \xi \zeta^{\prime} \cdot \gamma \rho . \kappa^{\prime}$. M. X.,$a \tau \lambda \gamma^{\prime}$.

\section{8}

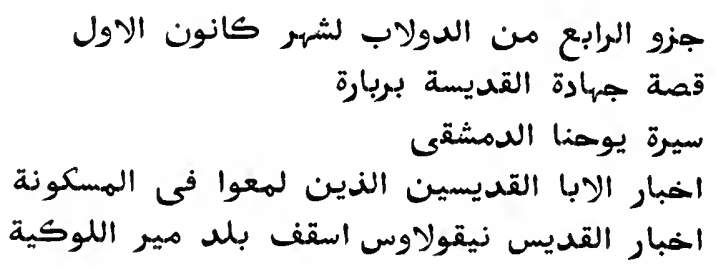




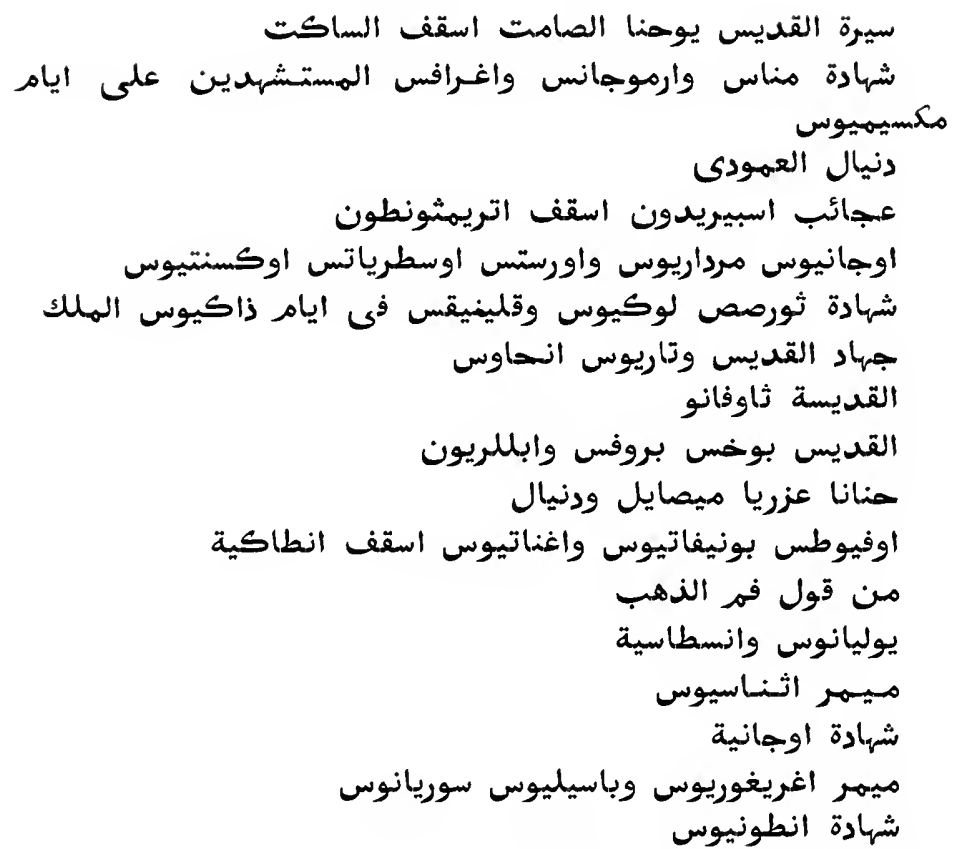

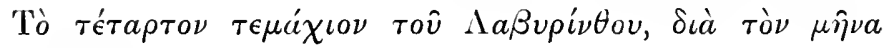
$\Delta \epsilon \kappa \epsilon \mu \beta$ piov.

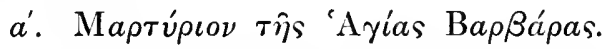

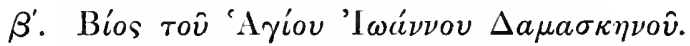

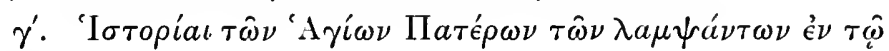
$\kappa o ́ \sigma \mu \omega$.

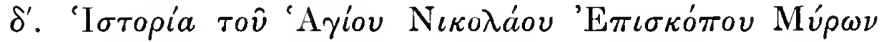

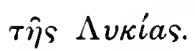

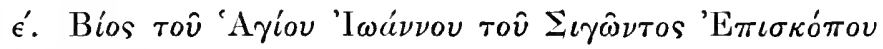

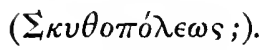

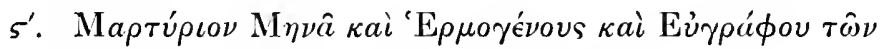

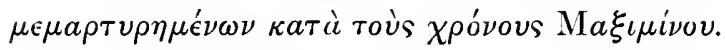

$\zeta^{\prime} . \Delta a \nu i \eta \lambda \Sigma^{\prime} \tau \nu \lambda i \tau \eta s$.

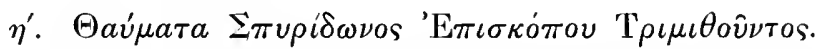

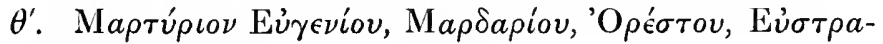

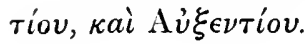




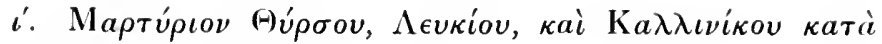

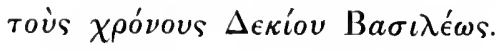

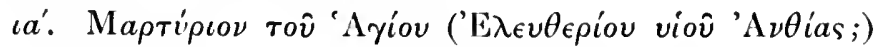

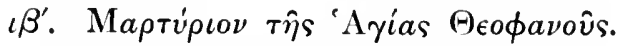

$\iota \gamma$ '. O $i^{\prime \prime}$ А

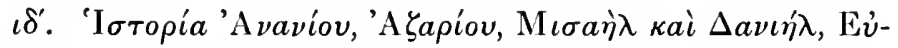

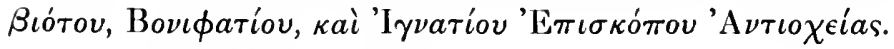

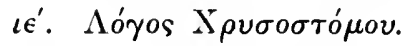

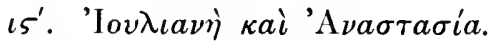

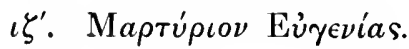

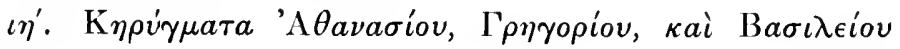

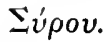

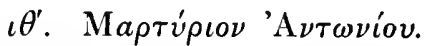

$\mathrm{X} \alpha^{\prime} \rho \tau \eta \varsigma^{\cdot} \lambda \varsigma^{\prime} \times \kappa^{\prime} \cdot \phi . \tau \delta^{\prime} \cdot \gamma \rho . \kappa a^{\prime} . \quad$ M. X.,$a \phi \pi \epsilon^{\prime}$.

\section{9}

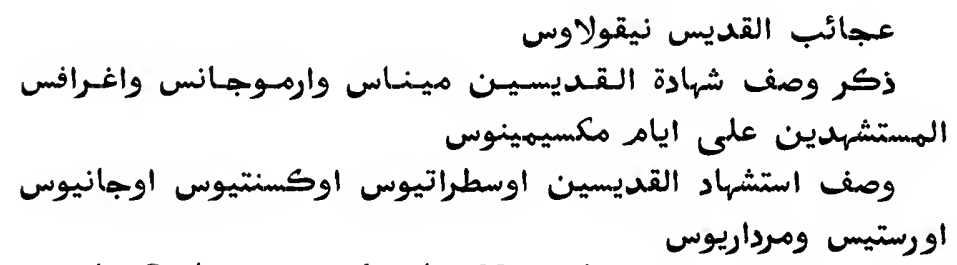

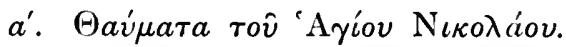

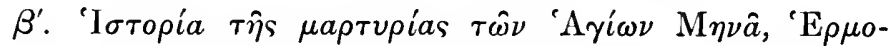

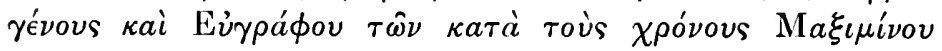

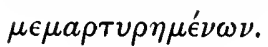

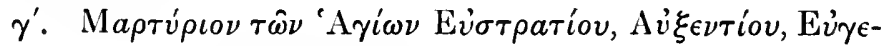

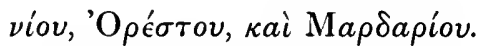

$\mathrm{X} a ́ \rho \tau \eta s^{\cdot} \kappa s^{\prime} \times \iota \eta^{\prime} \cdot \phi . \rho \nu \beta^{\prime} \cdot \gamma \rho . \iota \zeta^{\prime}$.

\section{0}

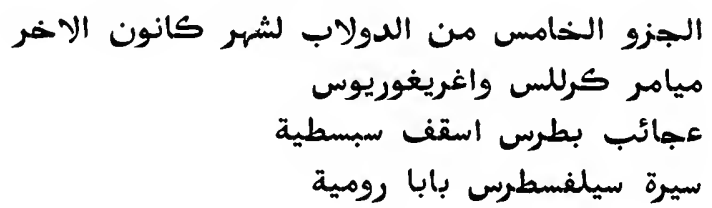




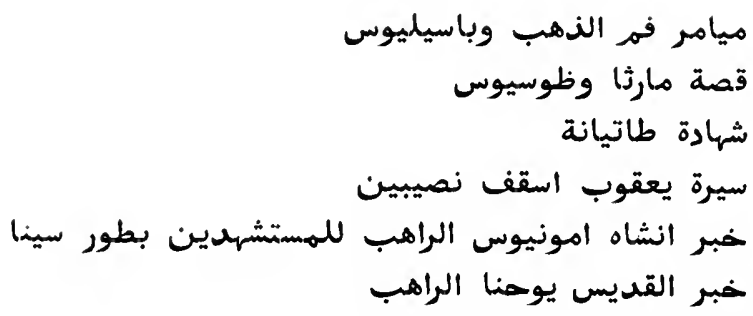

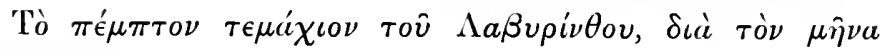
'Iavovapiov.

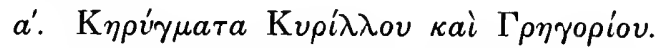

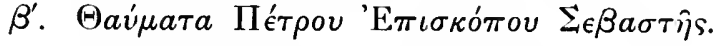

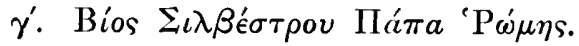

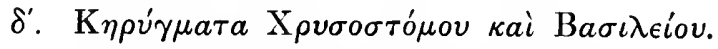

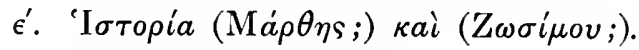

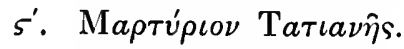

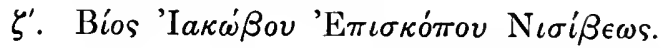

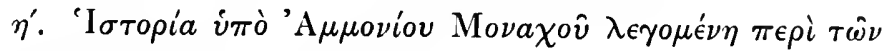

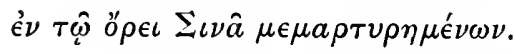

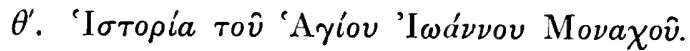

$\mathrm{X} \dot{\alpha} \rho \tau \eta s^{\cdot} \lambda \beta^{\prime} \times \kappa \beta^{\prime} \cdot \phi . \sigma \iota \epsilon^{\prime} \cdot \gamma \rho . \kappa a^{\prime}$.

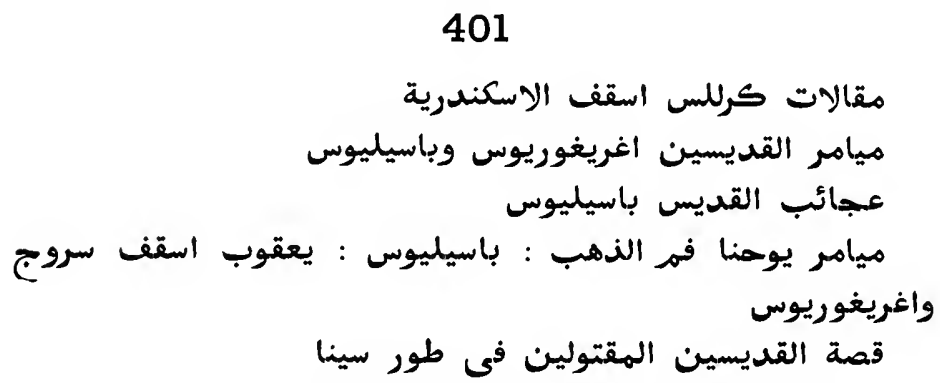

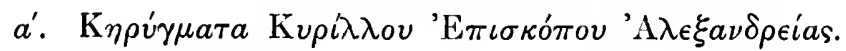

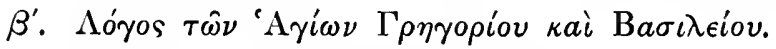

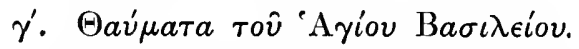

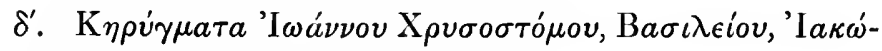

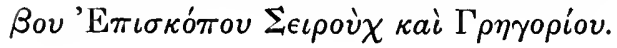




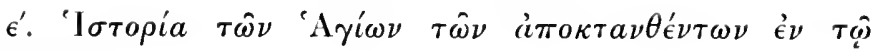

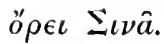

$\mathrm{X} a \dot{\alpha} \rho \tau \eta \varsigma^{\cdot} \lambda \delta^{\prime} \times \kappa \delta^{\prime} \cdot \phi . \sigma o \gamma^{\prime} \cdot \gamma \rho . \kappa a^{\prime}$.

\section{2}

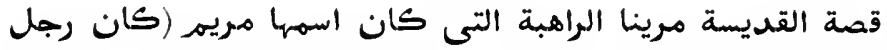

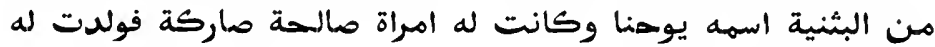

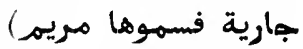

قصة الاب الخهير القديس الجماريليل ابا مرتينايوس الراهب

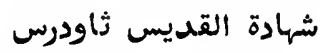

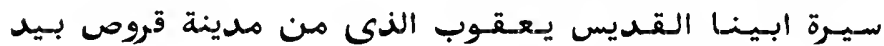

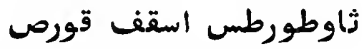

على وجود راس يوحنا الهعهدان

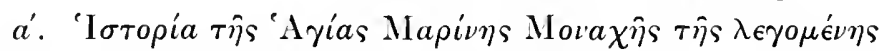

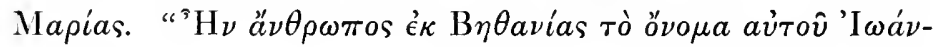

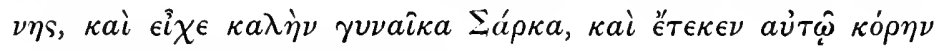

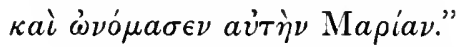

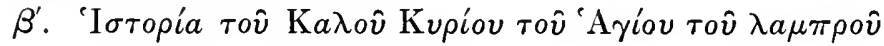

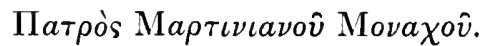

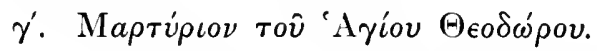

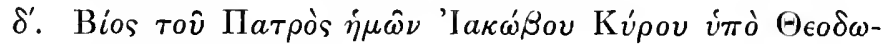
рйтоv 'Етıбко́тои Kúроv.

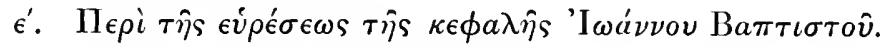

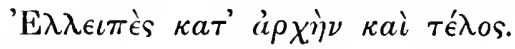

$\mathrm{X} a ́ \rho \tau \eta \varsigma^{\prime} \lambda a^{\prime} \times \kappa a^{\prime} \cdot \phi . \rho \kappa \theta^{\prime} \cdot \gamma \rho . \kappa^{\prime}$.

\section{3}

\section{ميامر بعض اللابيا}

شهادة القديس لليان : القديس نقفور : القديسة مرينا الراهبة :

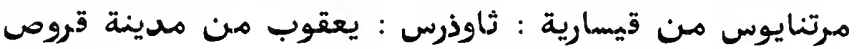

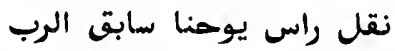

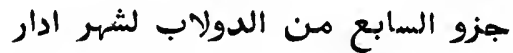

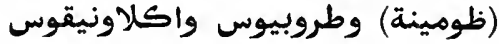


السسمـاريـة

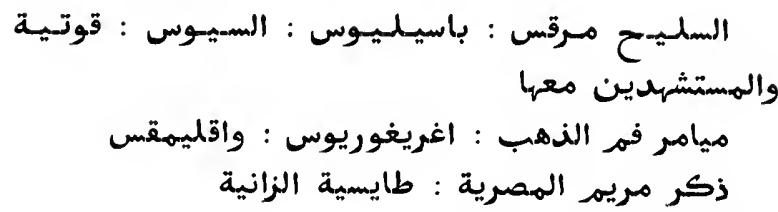

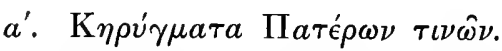
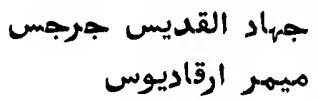

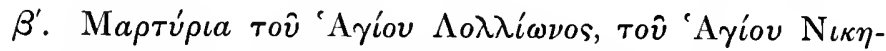

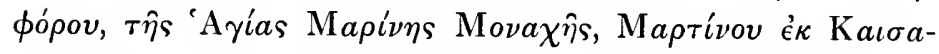

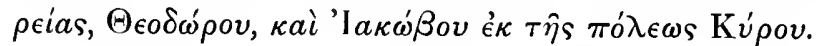

$\gamma^{\prime}$. 'А

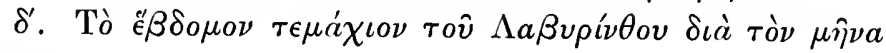
Maptíov.

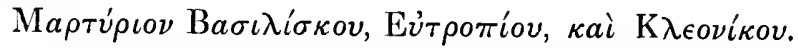

$\epsilon^{\prime}$. 'I бторі́a Ма́ркоv 'A

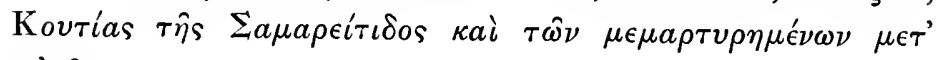
avivîs.

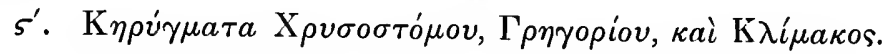

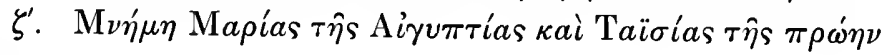

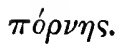

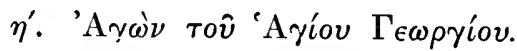

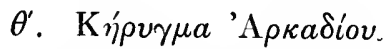

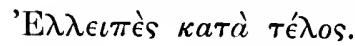

$\mathrm{X} a ́ \rho \tau \eta s^{\cdot} \lambda \delta^{\prime} \times \kappa \delta^{\prime} \cdot \phi . \tau \iota \epsilon^{\prime} \cdot \gamma \rho . \kappa a^{\prime}$.

'A $\phi \eta \rho \eta \mu \dot{c}_{\nu 0 \nu .}$

\section{4}

\section{5}

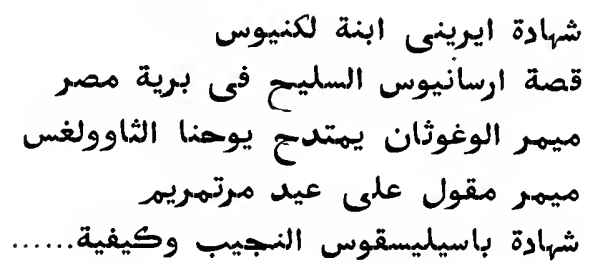




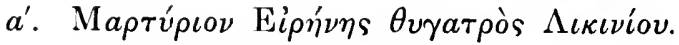

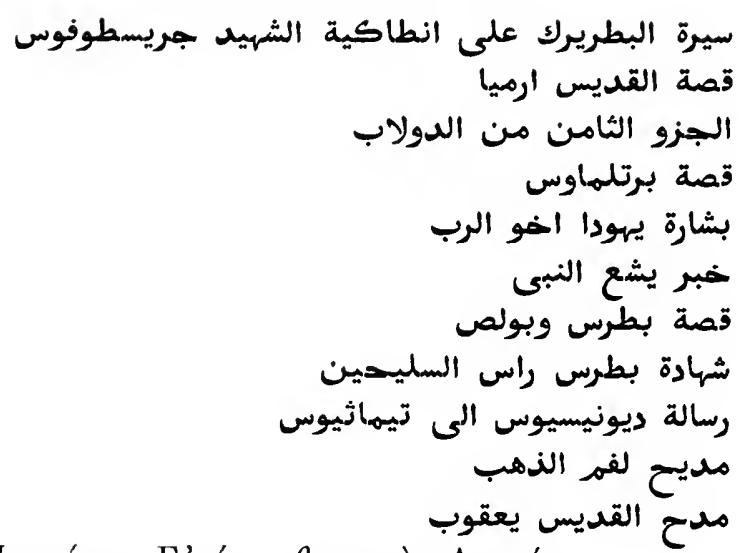

$\beta^{\prime}$. 'I $\sigma \tau o p i a$ 'A $\rho \sigma \epsilon \nu i o v$ 'A

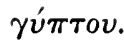

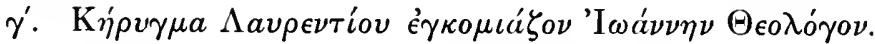

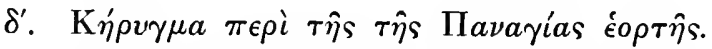

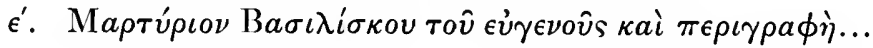

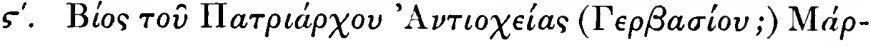
Tupos.

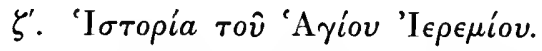

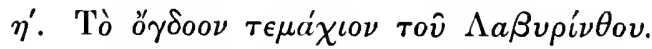

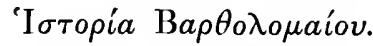

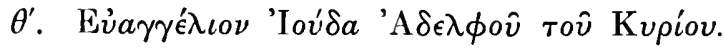

i. 'I $\sigma \tau o \rho i ́ a$ 'H $\sigma a i o v$ Профи́тov.

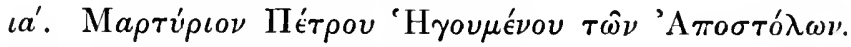

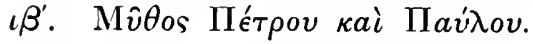

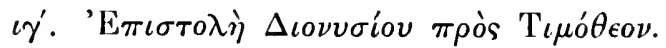

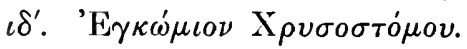

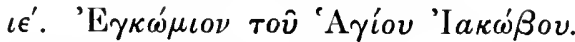

$\mathrm{X} \alpha \rho \tau \eta \varsigma^{\cdot} \lambda \gamma^{\prime} \times \kappa a^{\prime} \cdot \phi . \sigma \lambda \varsigma^{\prime}{ }^{\prime} \gamma \rho \kappa a^{\prime}$.

\section{6}

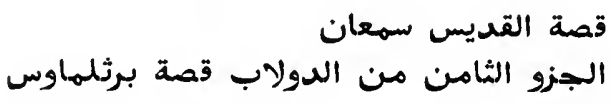




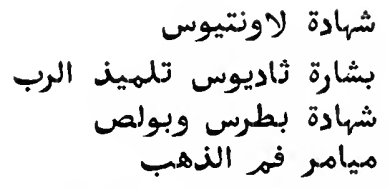

مدحة قالها يعقوب السمروجى للققديس يوحنا المهعمدان

$$
\text { قصة ثانية لشهادة بطرس ولهقوس }
$$

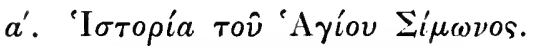

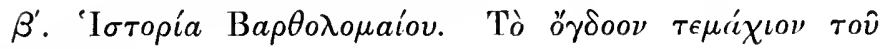
$\Lambda a \beta v \rho i \nu \theta o v$.

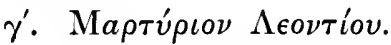

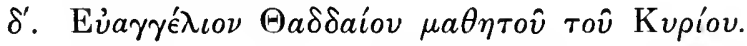

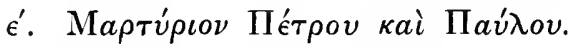

$5^{\prime}$. К

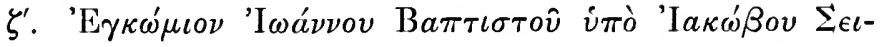

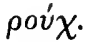

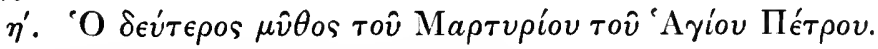

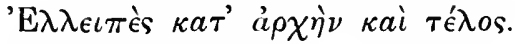

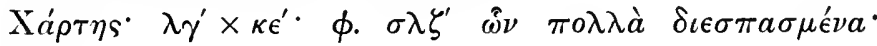
$\gamma \rho . \kappa a^{\prime}$.

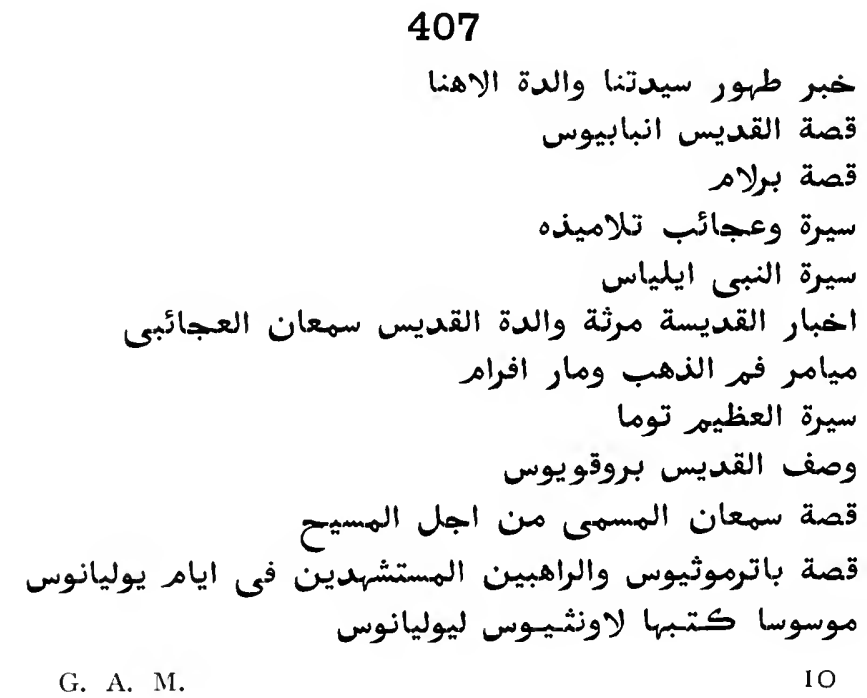




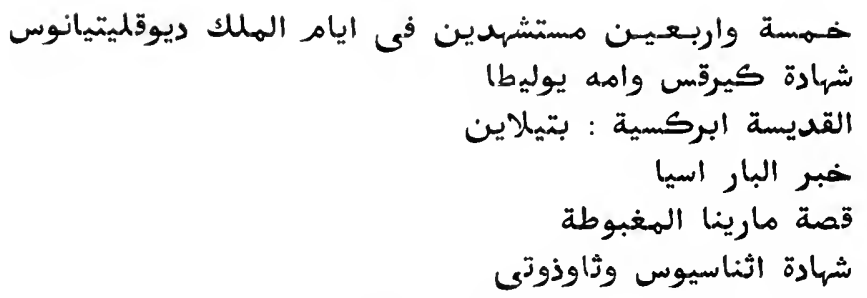

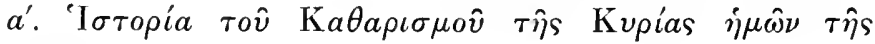

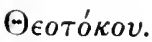

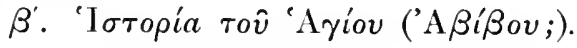

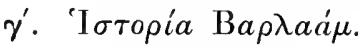

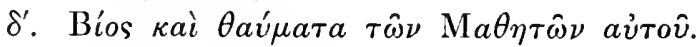

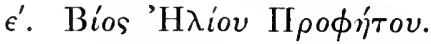

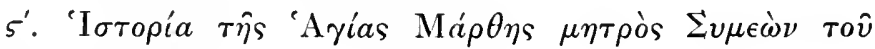

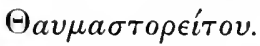

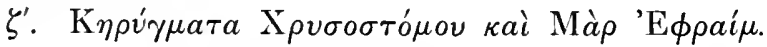

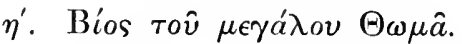

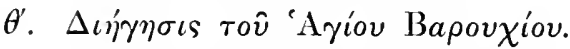

i. 'I

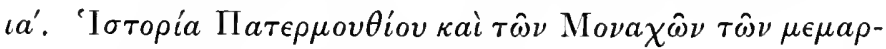

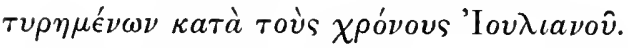

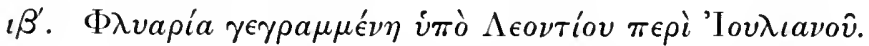

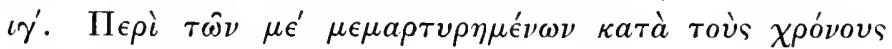

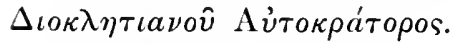

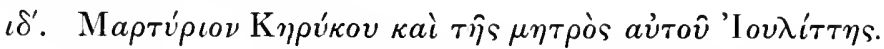

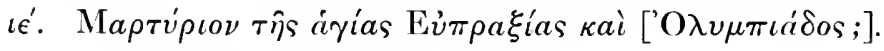

ıร'. 'I

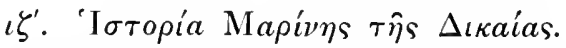

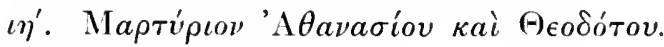

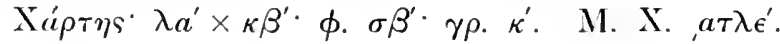

408

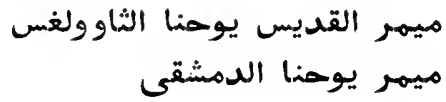




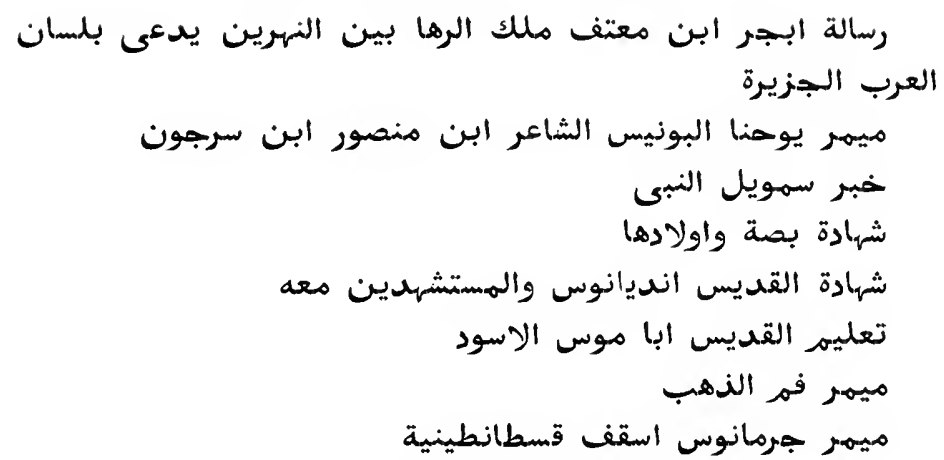

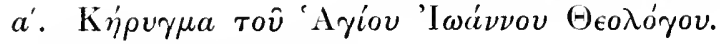

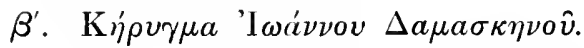

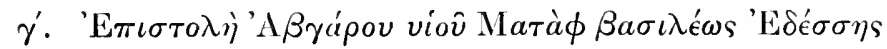

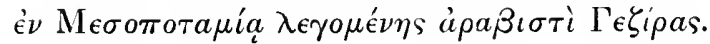

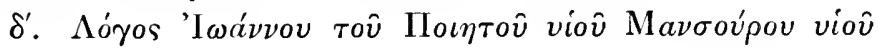
¿epriou.

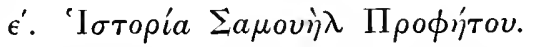

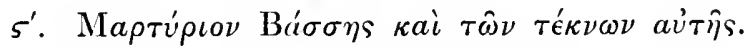

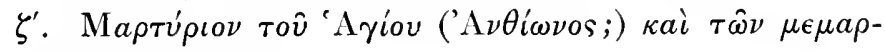

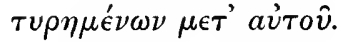

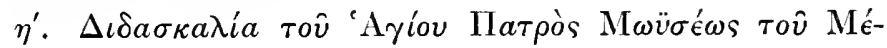
入avos.

$\theta^{\prime} . \mathrm{K} \dot{\eta} \rho v \gamma \mu \alpha$ Х $\rho v \sigma o \sigma \tau o ́ \mu o v$.

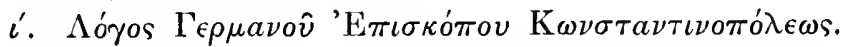

$\mathrm{X} a ́ \rho \tau \eta \varsigma^{\cdot} \lambda \varsigma^{\prime} \times \kappa \epsilon^{\prime} \cdot \phi . \rho \xi a^{\prime} \cdot \gamma \rho . \kappa a^{\prime} . \quad$ M. X.,$a \sigma \nu \eta^{\prime}$.

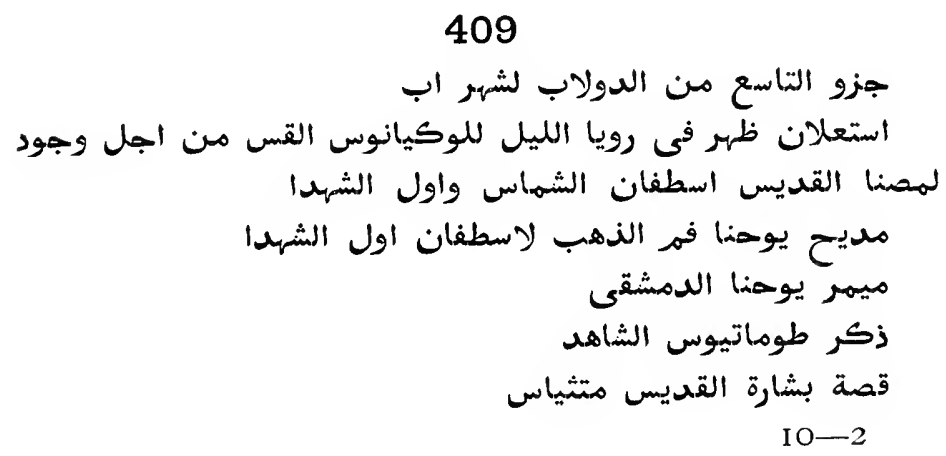




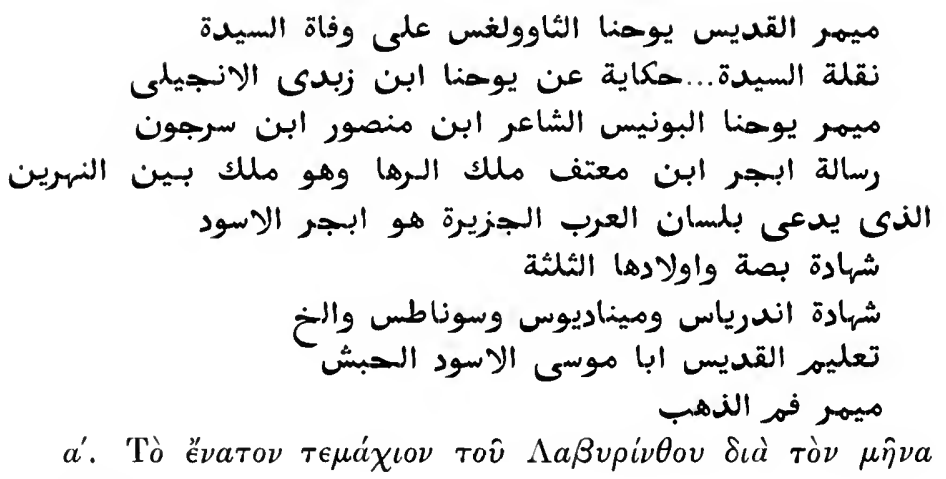
Aขंyoúgrov.

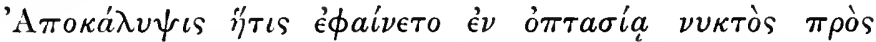

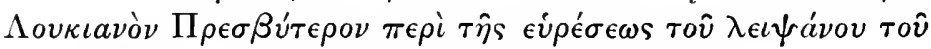

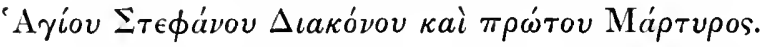

$\beta^{\prime}$. 'Е Хрvбобтó $\mu о v$.

$\gamma^{\prime} . \mathrm{K} \eta \dot{\rho} \rho \gamma \mu a$ 'I $\omega a \dot{\nu} \nu o v \quad \Delta a \mu a \sigma \kappa \eta \nu o \hat{v}$.

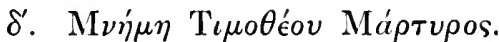

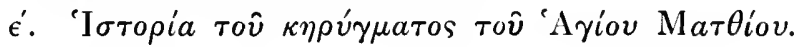

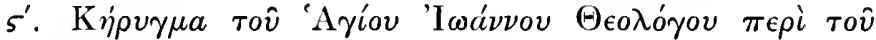

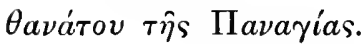

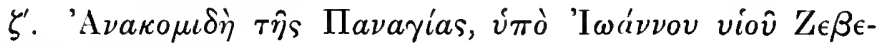

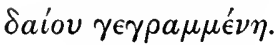

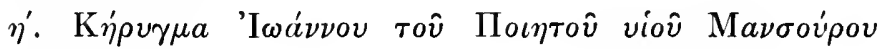

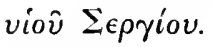

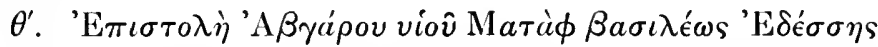

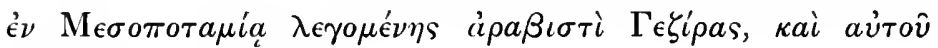

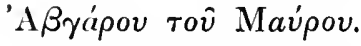

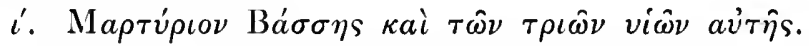

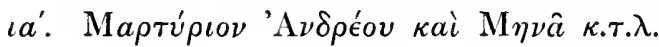

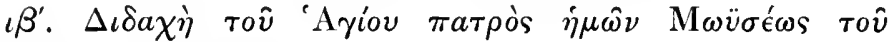

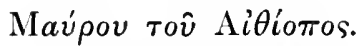

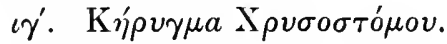

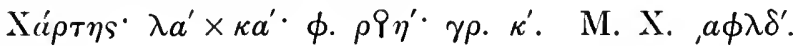




\section{0}

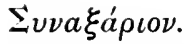

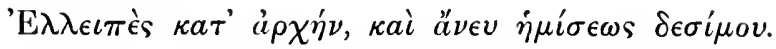

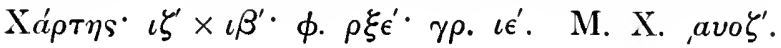

\section{1}

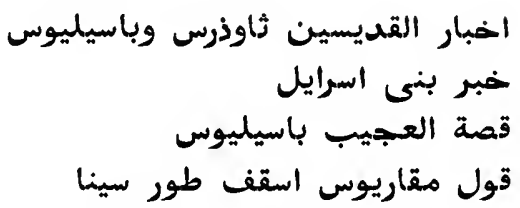

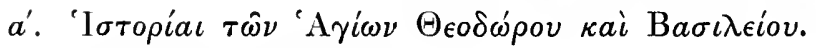

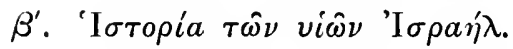

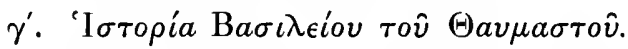

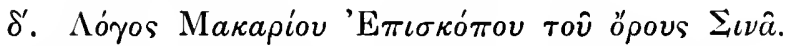

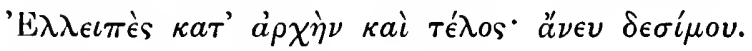

$\mathrm{X} a ́ \rho \tau \eta s^{\circ} \iota \eta^{\prime} \times \iota \delta^{\prime} \phi . \sigma \nu \epsilon^{\prime} \cdot \gamma \rho . \iota \epsilon^{\prime}$.

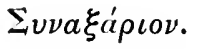

\section{2}

$\mathrm{X} a ́ \rho \tau \eta s^{\cdot} \kappa \delta^{\prime} \times \imath \eta^{\prime} \cdot \phi . \rho i \eta^{\prime} \gamma \rho . \iota \zeta^{\prime}$.

\section{3}

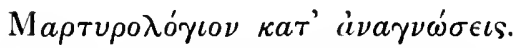

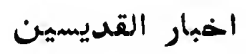

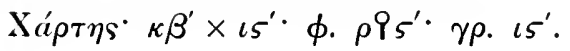

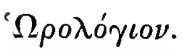

\section{4}

$\mathrm{X} \alpha \dot{\rho} \rho \eta \varsigma^{\circ} \kappa \gamma^{\prime} \times \iota \varsigma^{\prime} \cdot \phi . \rho \xi \epsilon^{\prime} \cdot \gamma \rho . \iota 5^{\prime}$.

415

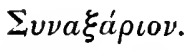

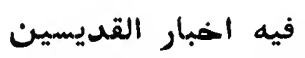

$\mathrm{X} a ́ \rho \tau \eta s^{\cdot} \kappa a^{\prime} \times \iota \delta^{\prime} \phi . \rho \mu \eta^{\prime} \gamma \rho . \iota \eta^{\prime}$. 


\section{6}

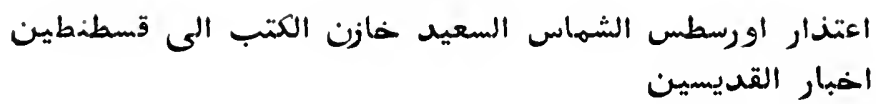

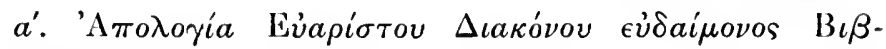

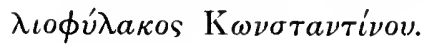

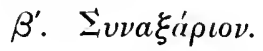

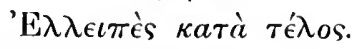

Хá $\rho \tau \eta s^{\cdot} \kappa \eta^{\prime} \times \kappa^{\prime} \cdot \phi . \sigma \epsilon^{\prime} \cdot \gamma \rho . \zeta^{\prime}$.

\section{7}

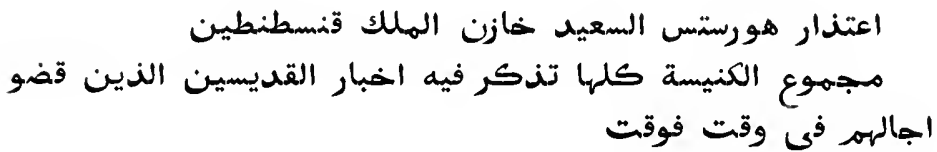

$a^{\prime}$. 'A

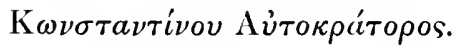

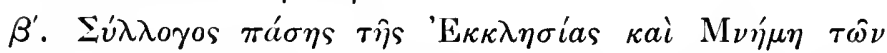

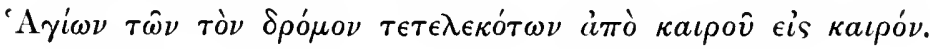

$\mathrm{X} a ́ \rho \tau \eta \varsigma^{\cdot} \kappa \alpha^{\prime} \times \iota \zeta^{\prime} \cdot \phi . \tau \gamma^{\prime} \gamma \rho . \kappa^{\prime}$. M. X. $a 9 \epsilon^{\prime}$.

'Spoגóyıov.

\section{8}

$\mathrm{X} a ́ \rho \tau \eta \varsigma^{\cdot} \kappa \delta^{\prime} \times \iota \varsigma^{\prime} \cdot \phi . \sigma \mu \zeta^{\prime} \cdot \gamma \rho . \kappa^{\prime}$.

\section{9}

$a^{\prime}$. Kavóvєs.

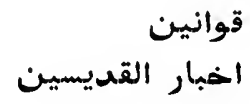

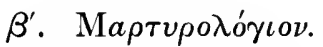

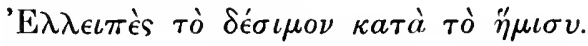

$\mathrm{X} a ́ \rho \tau \eta \varsigma^{\cdot} \kappa \varsigma^{\prime} \times \imath \eta^{\prime} \phi . \tau \iota \beta^{\prime} \gamma \rho \cdot \iota \zeta^{\prime}$.

\section{0}

اعتذار هورستس السعيد خازن الكتب الى قسطنطين

اخبار القديسين 
$a^{\prime}$. 'A $\mathrm{K} \omega \nu \sigma \tau a \nu \tau i \nu o v$.

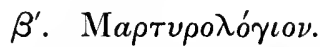

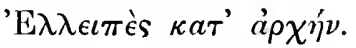

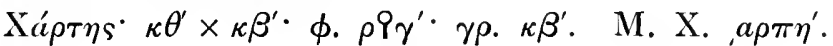

\section{1}

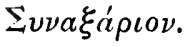

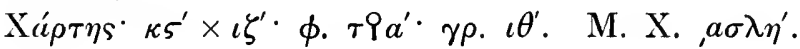

\section{2}

$\Sigma v \nu a \xi a ́ \rho \iota \nu$.

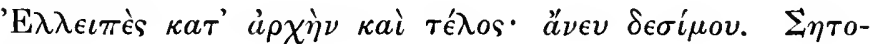

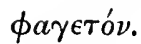

$\mathrm{X} \alpha \dot{\rho} \tau \eta \varsigma^{\cdot} \kappa \alpha^{\prime} \times \iota \delta^{\prime} \phi . \tau \xi \gamma^{\prime} \gamma \rho . \iota \theta^{\prime}$.

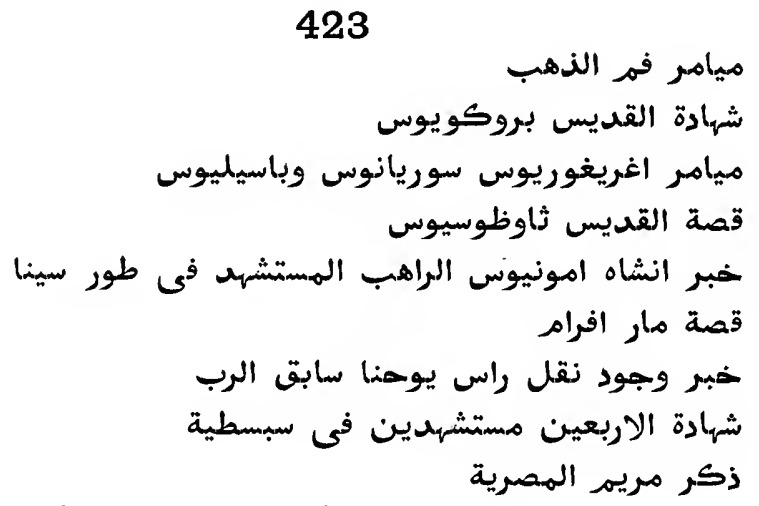

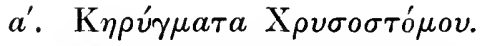

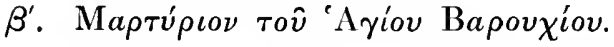

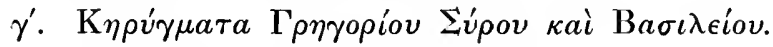

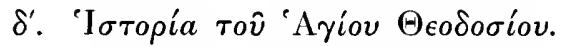

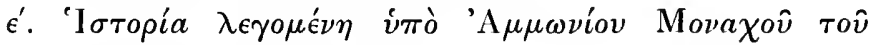

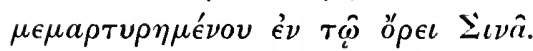

5'. Bios Мì 'Ефраíp. 


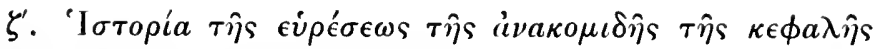

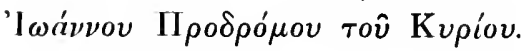

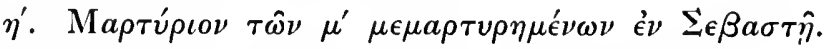

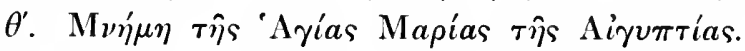

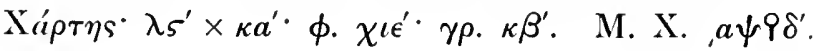

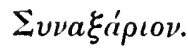

\section{4}

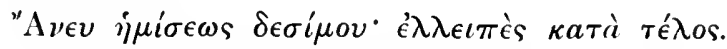

Х $\dot{\rho} \rho \eta \varsigma^{*} \iota^{\prime} \times \iota \beta^{\prime} \cdot \phi .95^{\prime} \cdot \gamma \rho . \iota^{\prime}$.

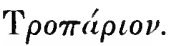

\section{5}

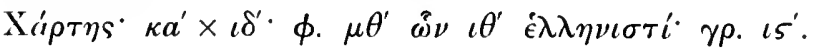

426

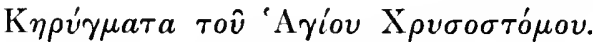

$$
\text { ميامر القديس فم الذهب }
$$

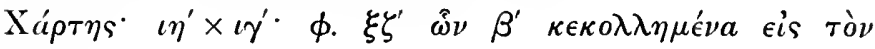
$\delta \epsilon^{\prime} \sigma \iota \mu \nu \nu^{\cdot} \gamma \rho . \iota a^{\prime}$.

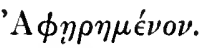

\section{7}

\section{8}

من قول مار افراه : هار اسحاق : فم الذهب : هقاريوس

قصة القديس يوحتا الرحوم إنرا

قصة سرابون

شهادة الحارث القديس شأهد بحبران وجميع اصحابه

من قول ابينا اسطبراط الفديس

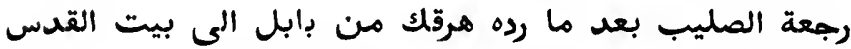

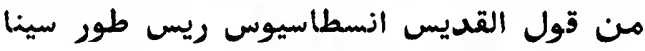

من قول القديس ابيفانيوس القديس 


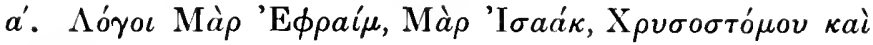
Макарі́о

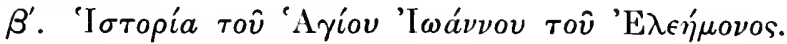

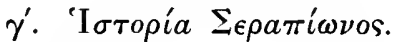

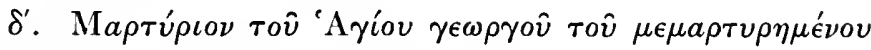

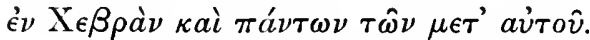

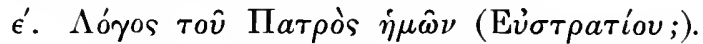

5'. 'А

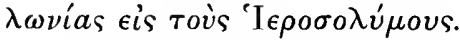

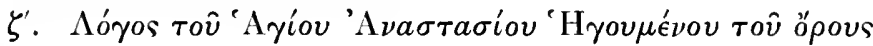
$\Sigma \iota \nu \hat{a}$

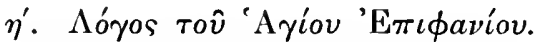

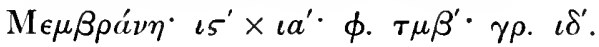

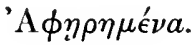

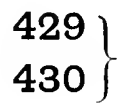

431

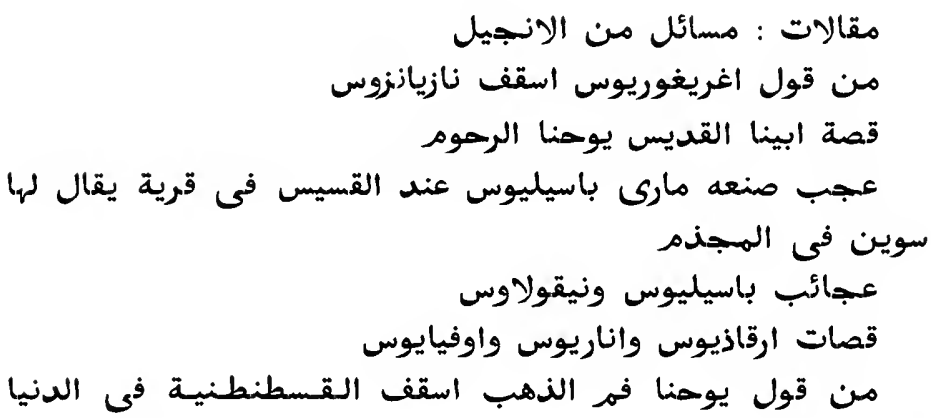

الداهرة - ماهن
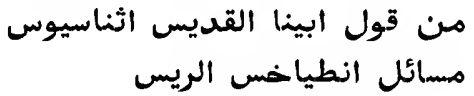

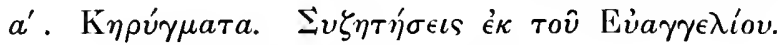

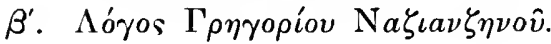

G. A. M. 


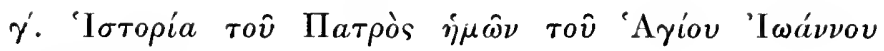
$\tau o \hat{v}$ 'E $\mathrm{\lambda} \epsilon \hat{\eta} \mu о \nu o s$.

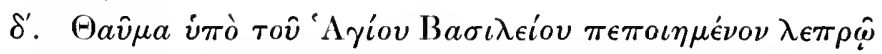

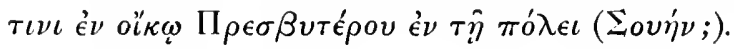

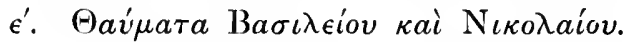

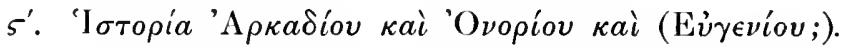

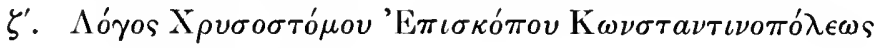

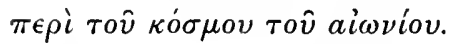

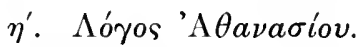

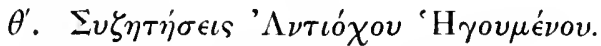

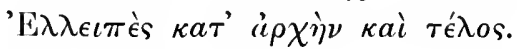

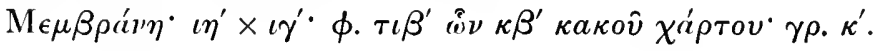

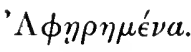

\section{2 \\ 433}

\section{4}

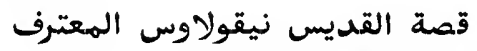

قول القديس افرام القديس فيسو

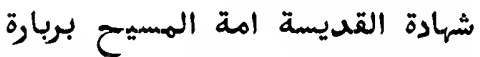

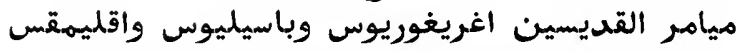

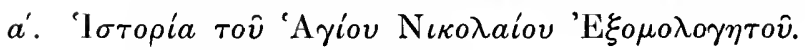

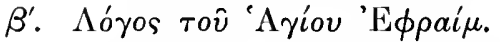

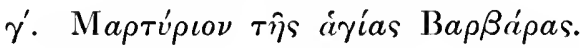

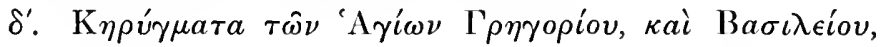
каі К入інакоs.

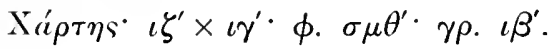

\section{5}

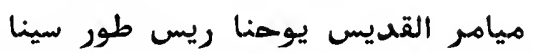

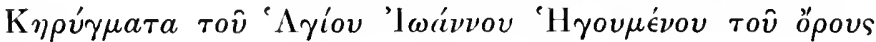
Sıvâ.

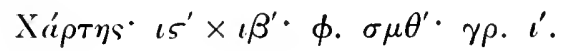




\section{6}

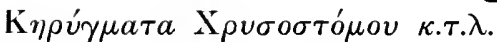

$$
\text { مقالات فم الذهب والخ }
$$

$\mathrm{X} a ́ \rho \tau \eta \varsigma^{*} \kappa^{\prime} \times \iota \delta^{\prime} \cdot \phi . \tau \pi \gamma^{\prime} \cdot \gamma \rho . \iota 5^{\prime}$.

\section{7}

اقوال فم الذهب : هوسى الذى فى الاسقيط : مار افرام وشعيا

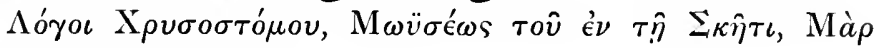

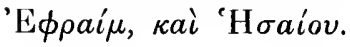

Хá $\rho \tau \eta \varsigma^{*} \kappa \epsilon^{\prime} \times \iota \varsigma^{\prime} \cdot \phi . \sigma \lambda \zeta^{\prime} \cdot \gamma \rho . \iota \epsilon^{\prime}$.

\section{8}

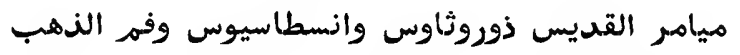

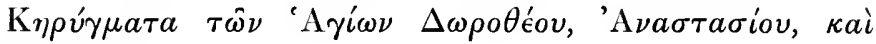
Хрvбобто́ноv.

$\mathrm{X} a \dot{\rho} \tau \eta \varsigma^{\cdot} \kappa a^{\prime} \times \iota \delta^{\prime} \cdot \phi . v \mu \eta^{\prime} \cdot \gamma \rho . \iota \delta^{\prime}$

\section{9}

الفردس المعقل وايضاح الاشجار والنبتات التى نصبها الله فيه

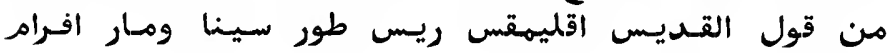
ومار باسيليوس والخ منول

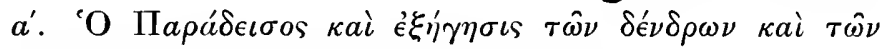

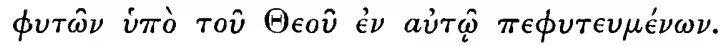

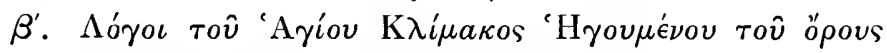

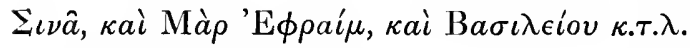

$\mathrm{X} a ́ \rho \tau \eta \varsigma^{\cdot} \kappa \beta^{\prime} \times \iota \delta^{\prime} \cdot \phi . \tau \nu \theta^{\prime} \cdot \gamma \rho . \kappa^{\prime}$. M. X. $a \sigma o \theta^{\prime}$.

\section{0}

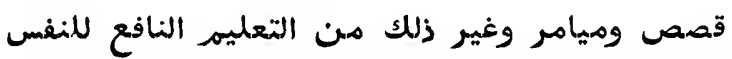

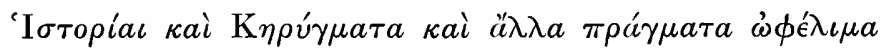
$\epsilon i s \tau \grave{\eta} \nu \psi v \chi \eta^{\prime} \nu$.

$\mathrm{X} a ́ \rho \tau \eta \varsigma^{\cdot} \kappa a^{\prime} \times \imath \delta^{\prime} \cdot \phi . \tau \kappa \eta^{\prime} \cdot \gamma \rho . \imath 5^{\prime}$.

$$
\text { I I }-2
$$




\section{1}

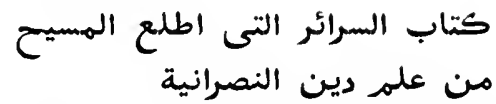

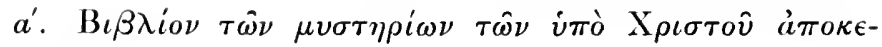
$\kappa a \lambda \nu \mu \mu \epsilon ́ \nu \omega \nu$.

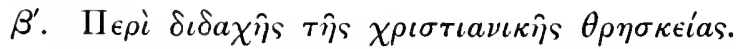

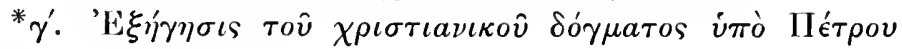
'Етıбко́тоv بيت راس.

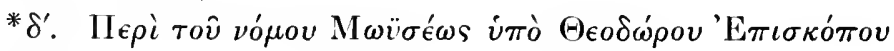
حران.

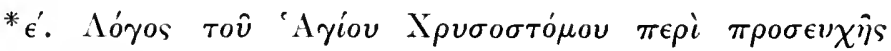

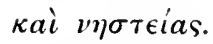

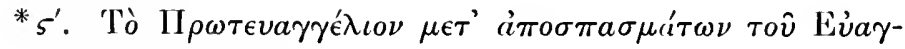

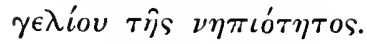

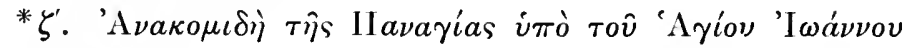

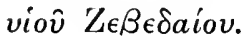

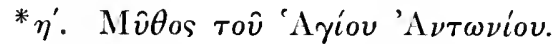

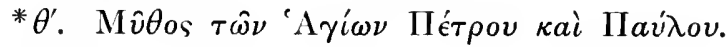

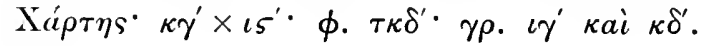

\section{2}
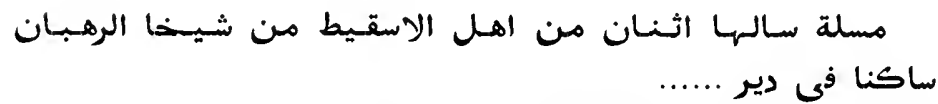

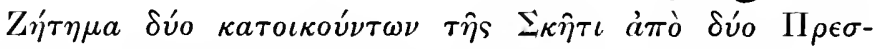

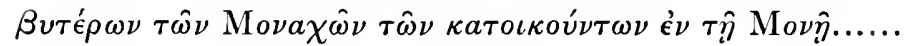

$\mathrm{X} a ́ \rho \tau \eta s^{\cdot} \iota \zeta^{\prime} \times \iota \beta^{\prime} \phi . \tau \iota \beta^{\prime} \gamma \rho . \iota \epsilon^{\prime}$.

\section{3}
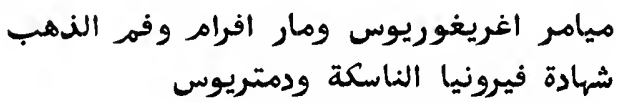

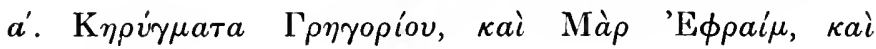
$\mathrm{X} \rho v \sigma o \sigma \tau o ́ \mu o v$. 


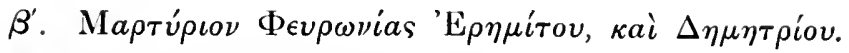

$\mathrm{X} a ́ \rho \tau \eta s^{\bullet} \kappa^{\prime} \times \iota \gamma^{\prime} \phi . \tau \iota^{\prime} \gamma \rho . \iota \delta^{\prime}$.

\section{4}

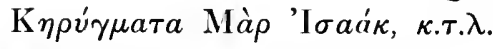

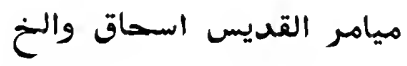

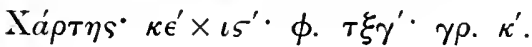

\section{5}

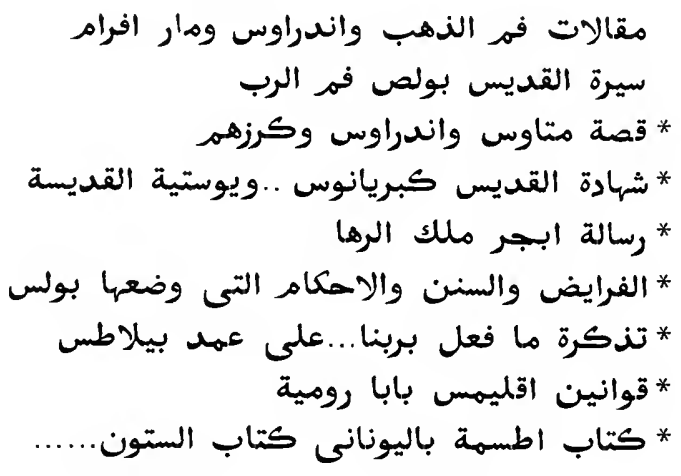

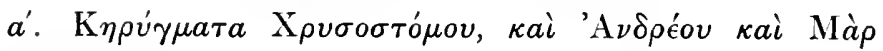

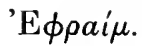

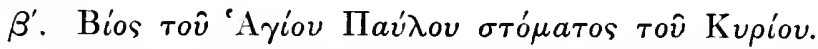

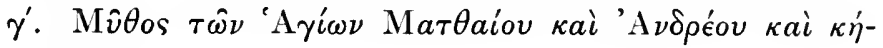

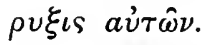

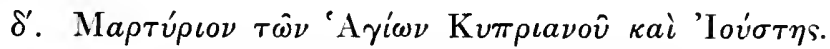

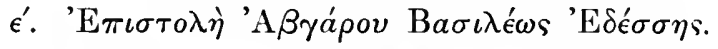

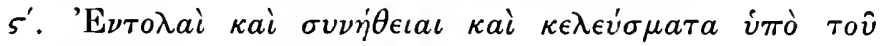

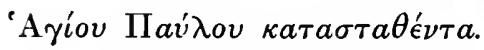

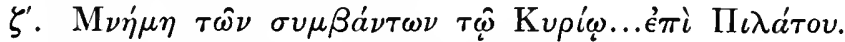

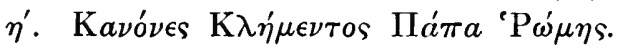

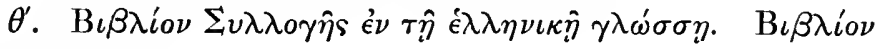

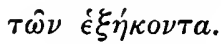

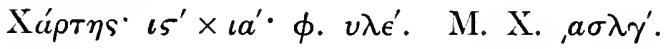




\section{6}

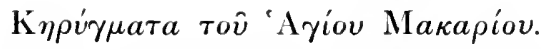

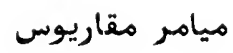

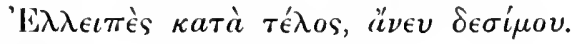

Хá $\rho \tau \eta \varsigma^{*} \kappa a^{\prime} \times i \delta^{\prime} \cdot \phi . \sigma \lambda^{\prime} \cdot \gamma \rho . \iota \zeta^{\prime}$.

\section{7}

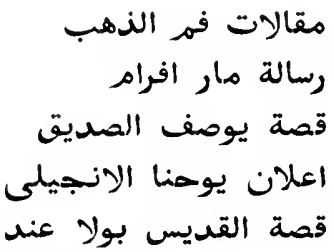

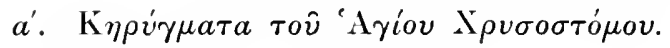

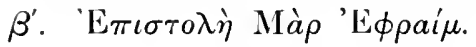

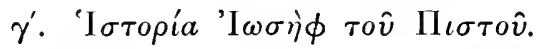

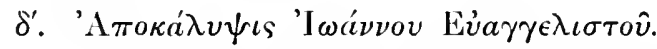

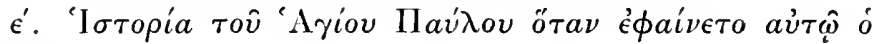
$\Delta \iota \dot{\beta} \beta$ oגos.

$\mathrm{X} \alpha \dot{\rho} \rho \eta \varsigma^{*} \kappa \epsilon^{\prime} \times \imath \varsigma^{\prime} \cdot \phi . \sigma \lambda \zeta^{\prime} \gamma \rho . \iota \zeta^{\prime}$.

\section{8}

$$
\text { قصة في برية الاسقيط ...... الداخلة في مغارة }
$$

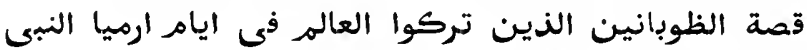

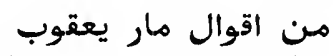

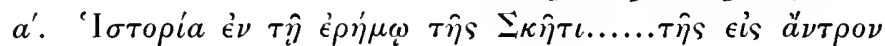
$\epsilon i \sigma \in \lambda \theta$ ov́ $\sigma \eta$ s.

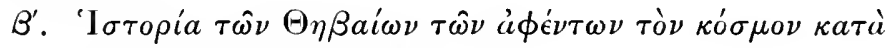

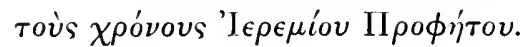

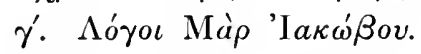

$\mathrm{X} \dot{\alpha} \rho \tau \eta \varsigma^{\cdot} \kappa a^{\prime} \times \iota \gamma^{\prime} \phi . \tau \kappa \gamma^{\prime} \gamma \rho . \kappa \gamma^{\prime}$.

\section{8 bis}

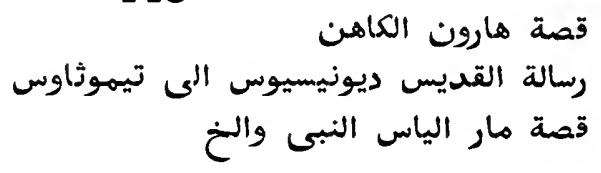




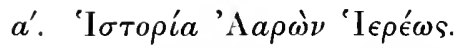

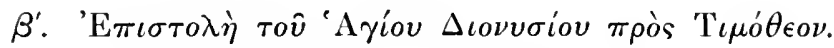

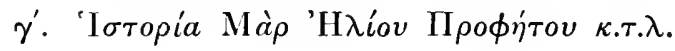

\section{9}

'А $\phi \eta \rho \eta \mu \operatorname{cov}^{\prime}$.

\section{0}

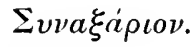

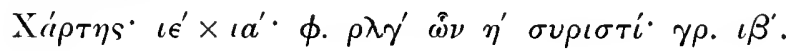

\section{1}

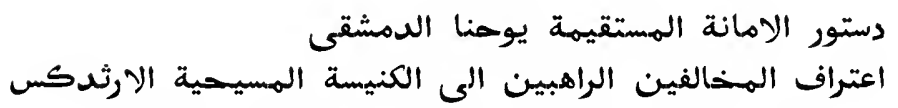

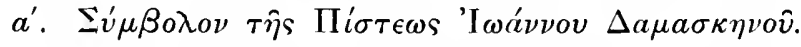

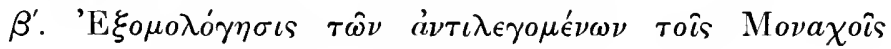

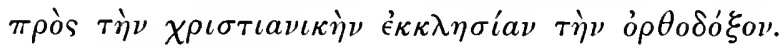

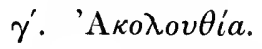

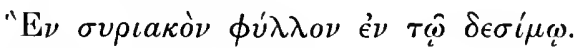

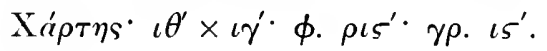

\section{2}

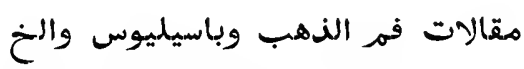

$\mathrm{K} \eta \rho \dot{\gamma} \mu a \tau a \mathrm{X} \rho v \sigma o \sigma \tau o ́ \mu o v, \kappa a \grave{~ B a \sigma \iota \lambda \epsilon i o v, ~ \kappa . \tau . \lambda . ~}$

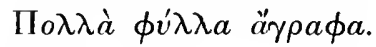

$\mathrm{X} \alpha \dot{\rho} \rho \eta \varsigma^{\circ} \iota \epsilon^{\prime} \times \iota^{\prime} \phi . \sigma \iota \gamma^{\prime} \gamma \rho . \iota \theta^{\prime}$. M. X. $a \psi \lambda \theta^{\prime}$.

\section{3}
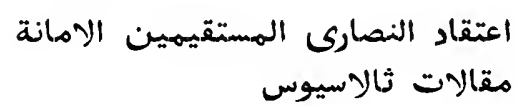

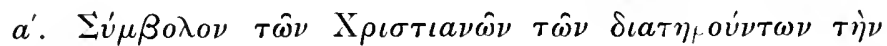
$\Pi i \sigma \tau \iota \nu$.

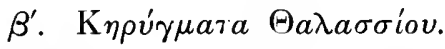

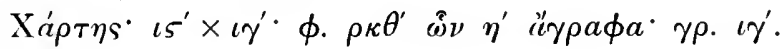




\section{4}

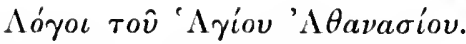

$$
\text { من قول القديس اثانسيوس }
$$

Х'́́ $\rho \tau \varsigma^{*} \kappa \epsilon^{\prime} \times \iota \zeta^{\prime} \cdot \phi . \pi \theta^{\prime} \cdot \gamma \rho . \kappa a^{\prime}$.

\section{5}
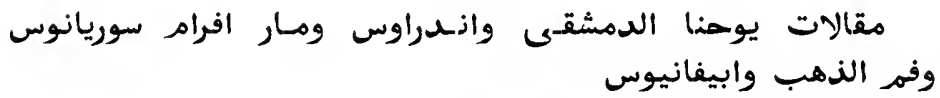

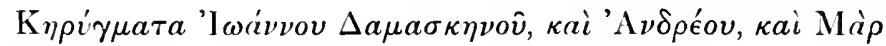

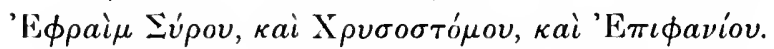

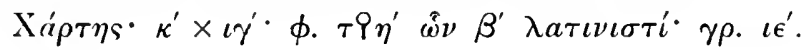

\section{6}

$$
\text { قصة نهمرود الهن كنعان اسكندر ذيى القرنين }
$$

اقوال مار اسحاق وهار افرام ويوحناف الناسك الفرني بطرك القسطنطنية

$$
\text { قوانين الاعتراف مار الفات ومري }
$$

قصة بنت اختى الآثراف مرتجن ابن اخر ارهيا النبى (استير الهاللة)

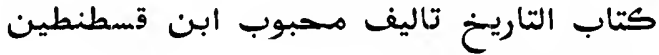

اخبار ابينا ابرهيم ابنيم

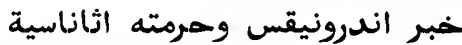

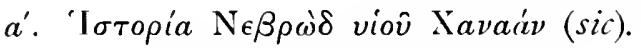

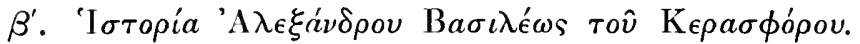

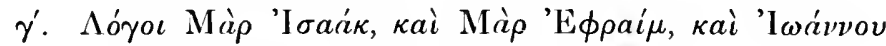

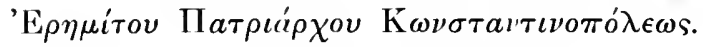

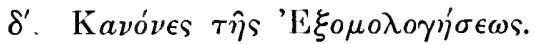

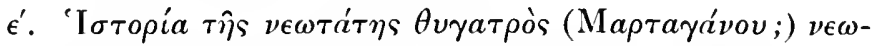

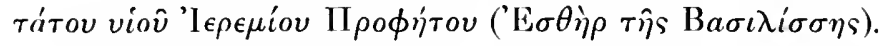

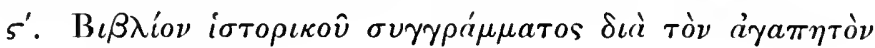
viòv $\mathrm{K} \omega \nu \sigma \tau a \nu \tau i ́ v o v$.

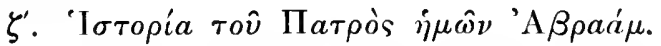

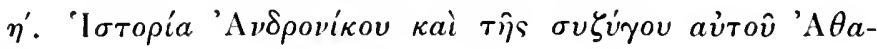
varias.

Х $а \dot{\rho} \tau \eta \varsigma^{\cdot} \kappa \varsigma^{\prime} \times \iota \eta^{\prime} \cdot \phi . \rho o \delta^{\prime} \cdot \gamma \rho . \iota \theta^{\prime}$. 


$$
\text { . }
$$




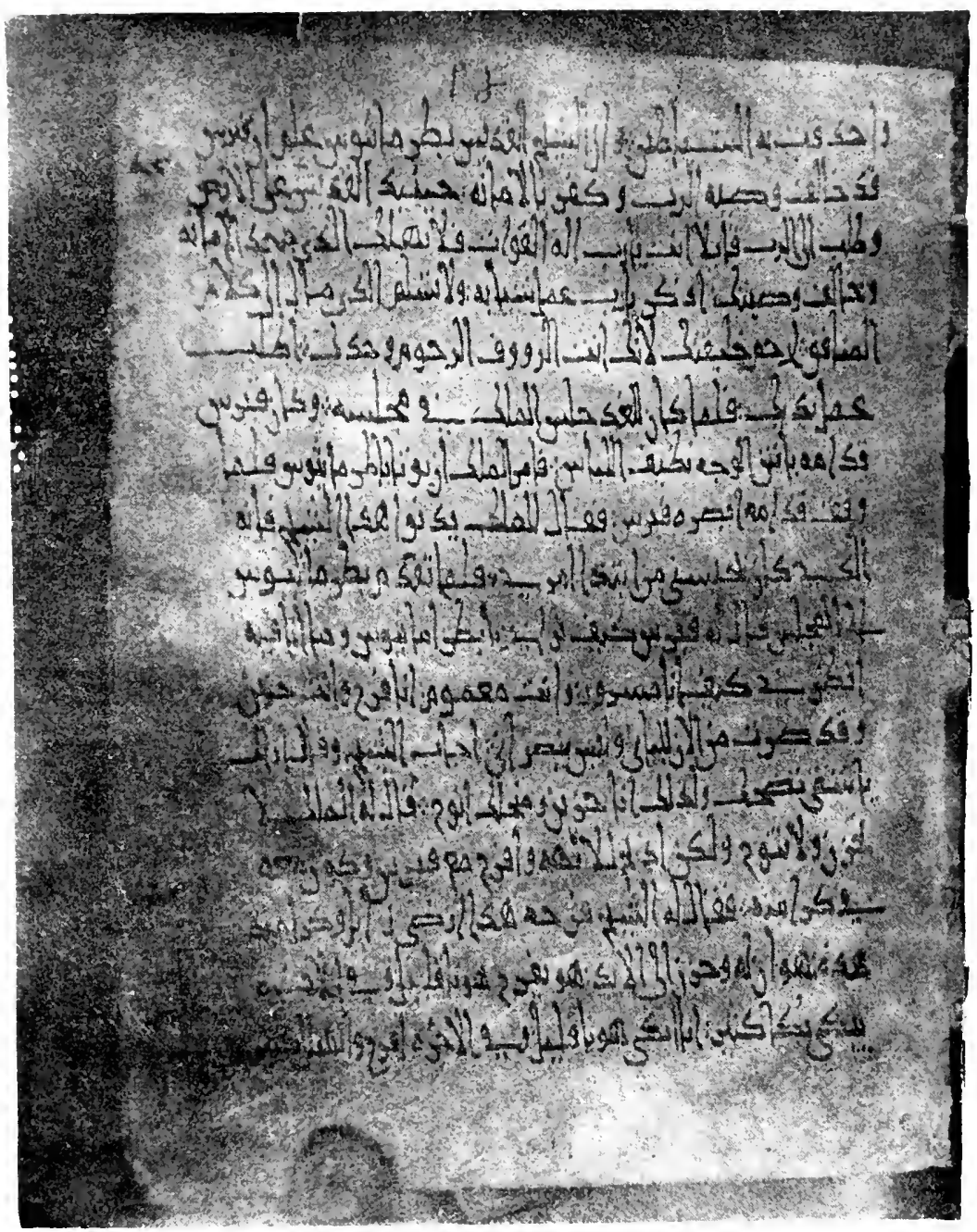

No 457
(From a thotograrth by M's Lewis) 


\section{$+457$}

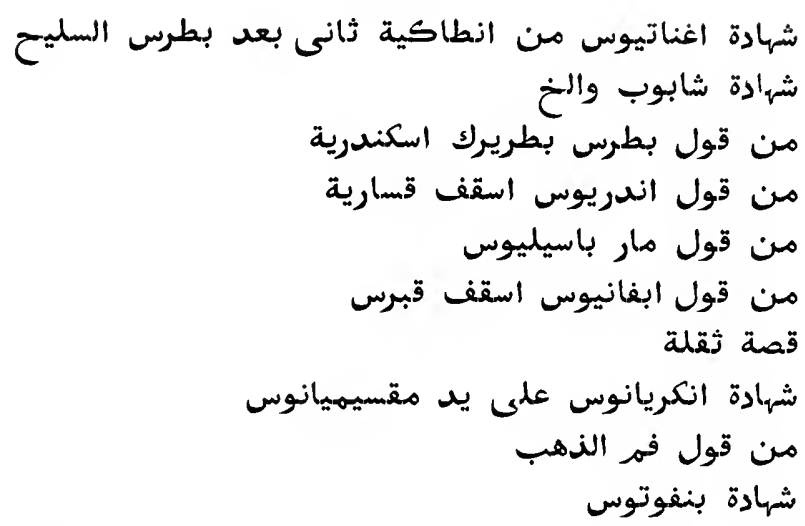

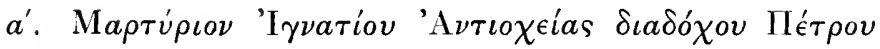
'A $\pi \circ \sigma \tau o ́ \lambda o v$.

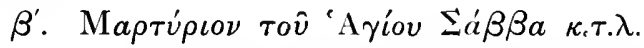

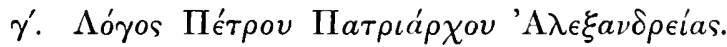

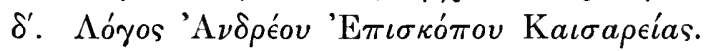

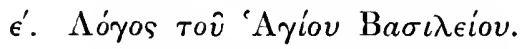

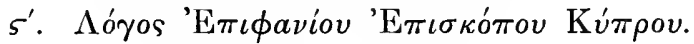

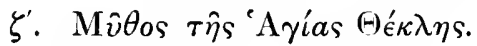

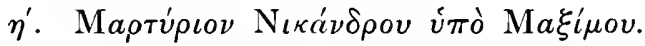

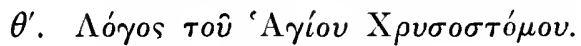

i. Maprúpıov Bovıфatíov.

$\mathrm{M} \epsilon \mu \beta \rho a^{\prime} \nu \eta^{\cdot} \kappa \beta^{\prime} \times \iota \zeta^{\prime} \phi . \rho \lambda \varsigma^{\prime} \gamma \rho . \kappa^{\prime}$.

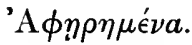

\section{$\left.\begin{array}{l}458 \\ 459\end{array}\right\}$}

$+460$
قصة اقليهس تلهميذ هار بطرس
قول مار يعقوب على مجمع الاول النى في في نيقية

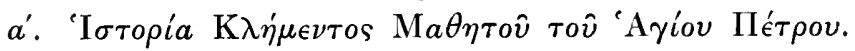

G. A. M. 


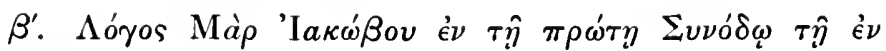
Nıкаía.

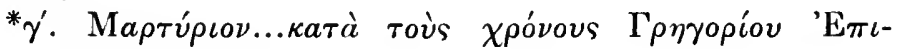

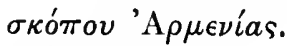

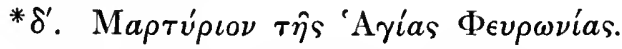

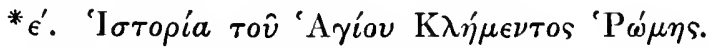

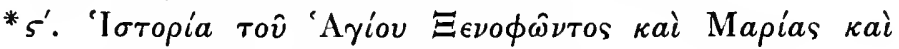

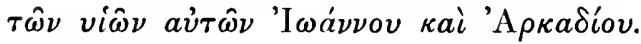

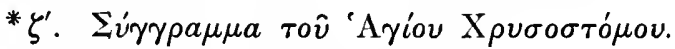

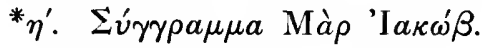

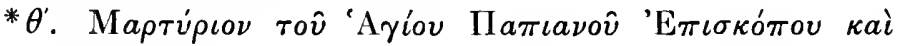
$\tau \rho \iota \hat{\omega} \nu \pi a i ́ \delta \omega \nu$.

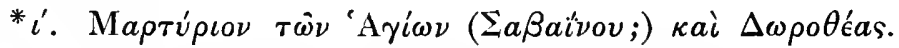

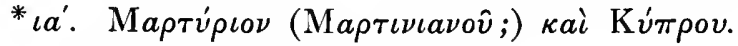

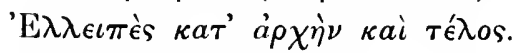

$\mathrm{M} \epsilon \mu \beta \rho \dot{a} \nu \eta^{\cdot} \kappa \beta^{\prime} \times \iota \epsilon^{\prime} \cdot \phi . \rho \delta^{\prime} \cdot \gamma \rho . \iota \eta^{\prime}$.

\section{1}

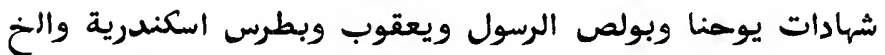

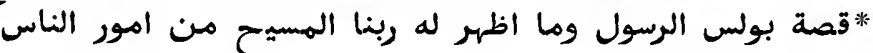

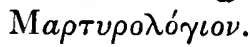

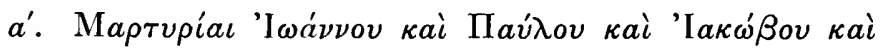
$\Pi_{\epsilon} \epsilon \rho o v ' \mathrm{~A} \lambda \epsilon \xi a \nu \delta \rho \epsilon i a s, \kappa \cdot \tau . \lambda$.

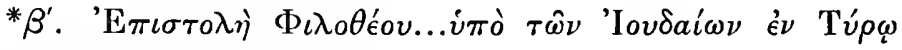

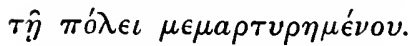

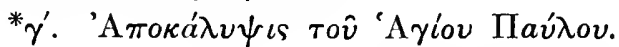

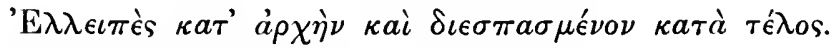

$\mathrm{M} \epsilon \mu \beta \rho a^{\prime} \nu \eta^{\cdot} \kappa a^{\prime} \times \iota \varsigma^{\prime} \cdot \phi . \rho \iota \zeta^{\prime} \cdot \gamma \rho . \kappa^{\prime}$.

\section{2 \\ 463 \\ 464 \\ 465}

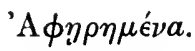




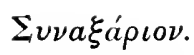

$+466$

$\Pi о \lambda \dot{v} \delta \iota \notin \phi \theta a \rho \mu \epsilon^{\prime} \nu o \nu$.

$\mathrm{X} a ́ \rho \tau \eta \varsigma^{\bullet} \iota 5^{\prime} \times \iota \beta^{\prime} \cdot \phi . \rho \pi \epsilon^{\prime} \cdot \gamma \rho . \iota \beta^{\prime}$.

\section{7}

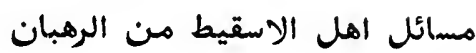

عظة

$a^{\prime} . \Sigma v \zeta \eta \tau \eta \dot{\eta} \sigma \epsilon \iota \varsigma \tau \hat{\omega} \nu \stackrel{\epsilon}{\gamma \chi \chi \omega \rho i \omega \nu} \tau \hat{\eta} \varsigma \Sigma_{\kappa} \hat{\eta} \tau \iota$ à $\pi \grave{o} \tau \hat{\omega} \nu \mathrm{Mo-}$ $\nu a \chi \hat{\omega} \nu$.

$\beta^{\prime} . \mathrm{K} \eta \dot{p} \rho \gamma \mu a^{\prime} \tau \iota \nu o s$.

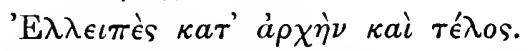

$\mathrm{X} a ́ \rho \tau \eta \varsigma^{\cdot} \iota \zeta^{\prime} \times \iota \gamma^{\prime} \phi . \sigma \nu a^{\prime} \cdot \gamma \rho . \quad \beta^{\prime}$.

\section{8}

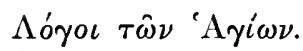

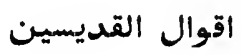

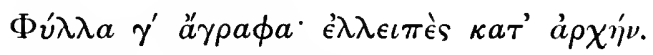

$\mathrm{X} \dot{\alpha} \rho \eta \eta \varsigma^{\prime} \iota \eta^{\prime} \times \iota \gamma^{\prime} \cdot \phi$. $\rho \kappa \epsilon^{\prime} \cdot \gamma \rho . \iota \gamma^{\prime}$.

\section{9}

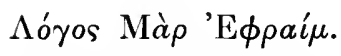

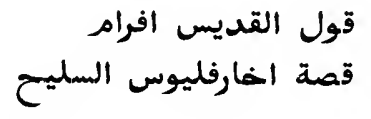

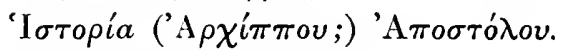

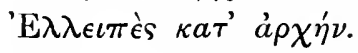

$\mathrm{X} \dot{\alpha} \rho \tau \eta \varsigma^{\cdot} \iota \zeta^{\prime} \times \iota \beta^{\prime} \cdot \phi . \rho \iota \zeta^{\prime} \gamma \rho . \iota \zeta^{\prime}$.

\section{0}

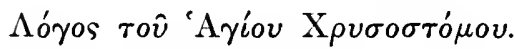

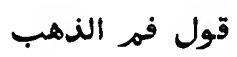

$\mathrm{X} a ́ \rho \tau \eta \varsigma^{\cdot} \iota \zeta^{\prime} \times \iota \beta^{\prime} \cdot \phi . \rho \zeta^{\prime} \cdot \gamma \rho . \iota \gamma^{\prime}$.

\section{1}

$\Sigma \chi o ́ \lambda \iota a$.

تغاسير

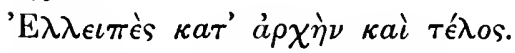

$\mathrm{X} \dot{a} \rho \tau \eta \varsigma^{\cdot} \iota \varsigma^{\prime} \times \iota \beta^{\prime} \cdot \phi . \sigma \beta^{\prime} \cdot \gamma \rho$. $\iota \gamma^{\prime}$. 


\section{2}

$\mathrm{O} i \grave{e} \pi \tau \dot{a} \mathrm{~N}$ aoì $\tau o \hat{v} \phi \omega \tau o ́ s$.

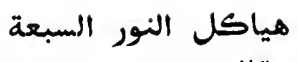

مقالات

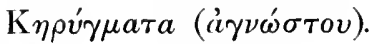

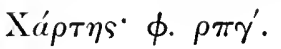

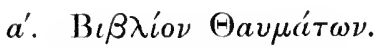

473

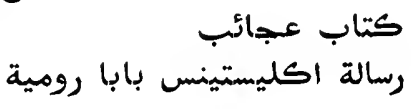

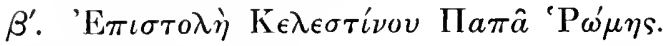

$\Pi о \lambda \dot{v} \delta \iota \epsilon \phi \theta a \rho \mu e^{\prime} \nu \nu$.

Х $\alpha \dot{\rho} \tau \eta \varsigma^{\cdot} \iota \varsigma^{\prime} \times \imath \gamma^{\prime} \cdot \phi . \pi \zeta^{\prime} \cdot \gamma \rho . \imath \beta^{\prime}$.

\section{4}

من قول القديس كرللس قاله على دخول ربنا الى الكنيسة لمصر اربعين يوماً

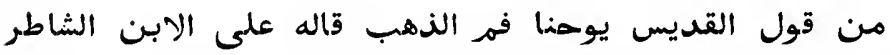

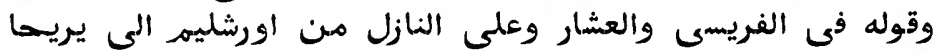

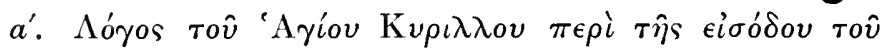

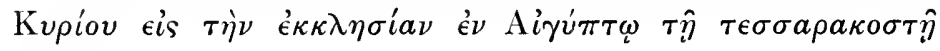
$\dot{\eta} \mu e^{\prime} \rho a$.

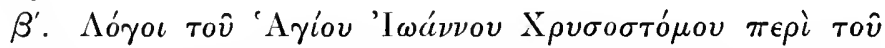

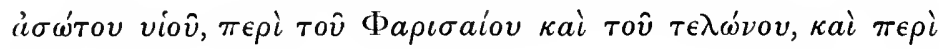

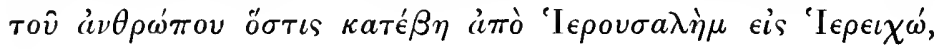
$\kappa, \tau, \lambda$.

$\mathrm{X} a \dot{\rho} \rho \tau \varsigma^{\cdot} \kappa a^{\prime} \times \iota \beta^{\prime} \cdot \phi . \tau \kappa \eta^{\prime} \cdot \gamma \rho . \iota \eta^{\prime}$.

\section{5}

مقالات مار افوام واغريغوريوس وفم الذهب

قصة مقيلونثيوس القديس

شهادة بنوفاتيوس

قصة الرسل بطرس وبولص بنوس 


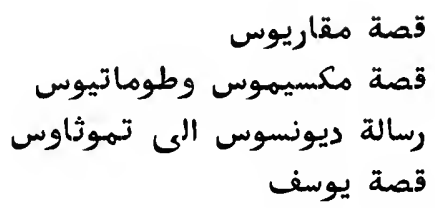

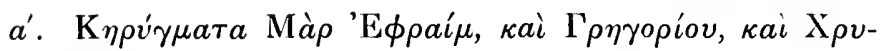

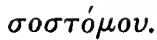

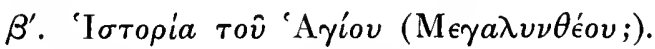

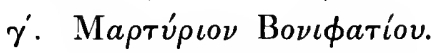

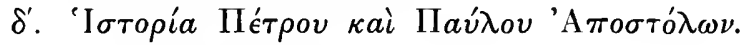

é. 'I

5'. 'I

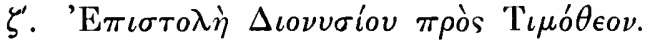

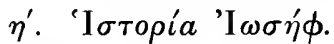

$\mathrm{X} a ́ \rho \tau \eta s^{\cdot} \kappa a^{\prime} \times \iota a^{\prime} \phi . \sigma o \beta^{\prime} \gamma \rho . \iota \zeta^{\prime}$.

\section{6}

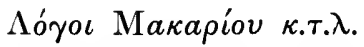

اقوال مقاريوس والخ

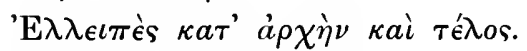

$\mathrm{X} a ́ \rho \tau \eta s^{*} \kappa \epsilon^{\prime} \times \iota \zeta^{\prime} \cdot \phi . \sigma^{\prime} \cdot \gamma \rho . \kappa^{\prime}$.

\section{7}

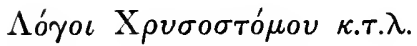

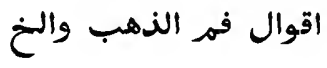

$\mathrm{X} a ́ \rho \tau \eta \varsigma^{\cdot} \kappa a^{\prime} \times \iota \delta^{\prime} \phi \phi . \rho \kappa \varsigma^{\prime} \gamma \rho . \iota \zeta^{\prime}$.

\section{$+478$}

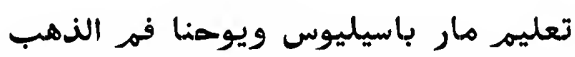

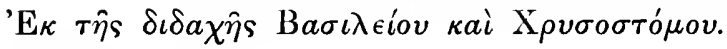

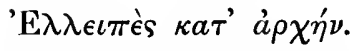

$\mathrm{X} \alpha \dot{\rho} \tau \eta \varsigma^{\cdot} \kappa \alpha^{\prime} \times \iota \delta^{\prime} \cdot \phi . \sigma \xi^{\prime} \gamma \rho . \iota \epsilon^{\prime}$.

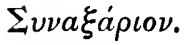

\section{9}

$\mathrm{X} a ́ \rho \tau \eta \varsigma^{*} \iota \varsigma^{\prime} \times \iota a^{\prime} \phi . \pi^{\prime} \dot{\omega} \nu \zeta^{\prime} a^{\prime} \gamma \rho a \pi \tau a^{\cdot} \gamma \rho . \kappa^{\prime}$. 


\section{0}

$\Sigma_{\chi o ́} \lambda \iota a(\dot{a} \gamma \nu \omega \dot{\sigma} \sigma o v)$.

تفاسير

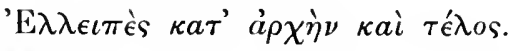

$\mathrm{X} \alpha \dot{\rho} \tau \eta \varsigma^{\cdot} \kappa \beta^{\prime} \times \iota \epsilon^{\prime} \cdot \phi \cdot \rho \lambda^{\prime} \cdot \gamma \rho \cdot \iota \zeta^{\prime}$.

\section{1}

انسطاسيوس اجوبة عن الهسائل

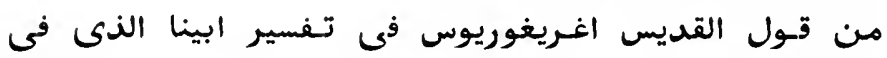

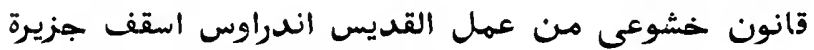

a'. 'A

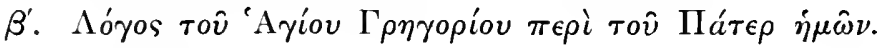

$\gamma^{\prime} . \Sigma v \zeta \eta \tau \eta \dot{\sigma} \sigma \iota s$.

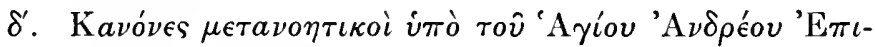

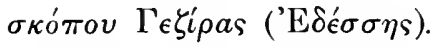

$\mathrm{X} \alpha \dot{\rho} \rho \tau \eta \varsigma^{\cdot} \kappa 5^{\prime} \times 15^{\prime} \cdot \phi . \tau \lambda \delta^{\prime} \cdot \gamma \rho . \kappa a^{\prime}$.

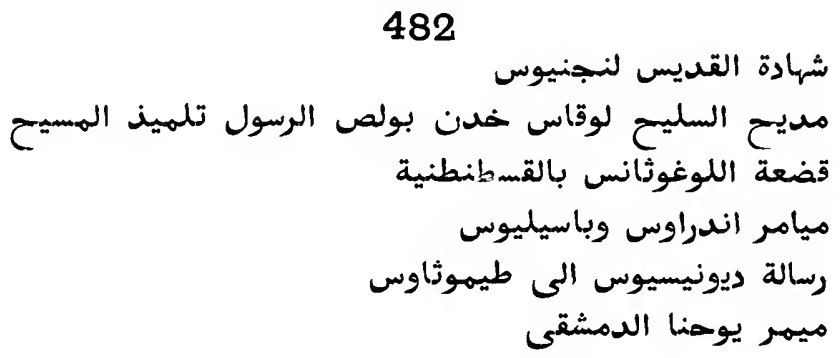

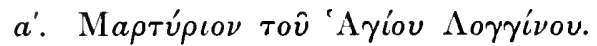

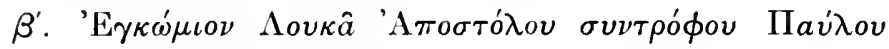
'А

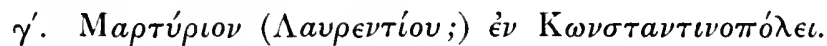

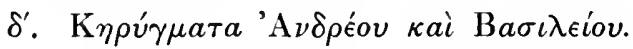

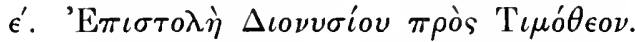

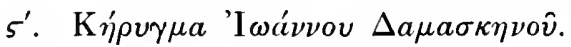

$\mathrm{X} a ́ \rho \tau \eta \varsigma^{\cdot} \kappa \delta^{\prime} \times \iota \epsilon^{\prime} \cdot \phi, \sigma \rho \zeta^{\prime} \cdot \gamma \rho . \kappa^{\prime}$. 


\section{3}

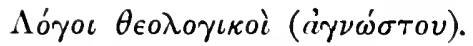

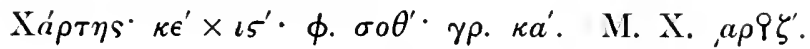

¿vvaḱápıov.

\section{4}

$\mathrm{X} a \dot{\rho} \tau \eta \varsigma^{\cdot} \kappa a^{\prime} \times \iota \delta^{\prime} \cdot \phi . \rho \nu \beta^{\prime} \cdot \gamma \rho . \iota \zeta^{\prime}$.

\section{5}

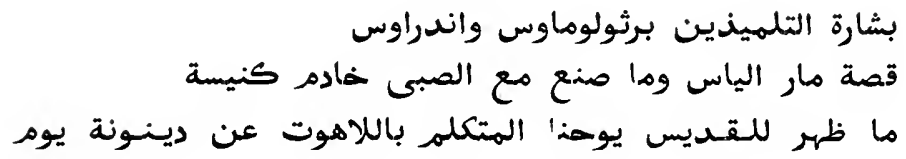

رسالة ابهر ابن هعهوق ملك الرها وهو بين النهرين ويقال

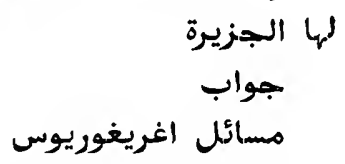

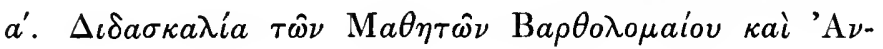
$\delta \rho$ ćov.

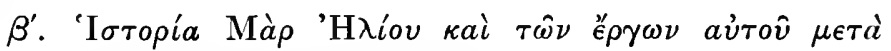

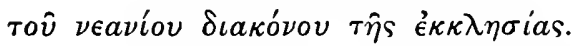

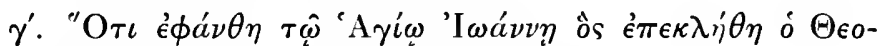

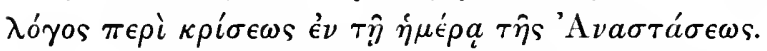

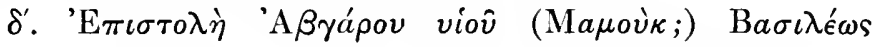

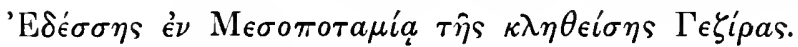

$\epsilon^{\prime}$. 'A $\mathrm{A} a \dot{\nu} \nu \eta \eta \iota \varsigma$.

5'. $\Sigma v \zeta \eta \tau \eta \dot{\sigma \epsilon \epsilon \varsigma} \Gamma \rho \eta \gamma o \rho i o v$.

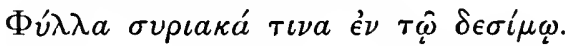

$\mathrm{X} a \rho \tau \eta s^{\cdot} \iota 5^{\prime} \times \iota \beta^{\prime} \cdot \phi . \rho \lambda \gamma^{\prime} \gamma \rho . \iota \gamma^{\prime}$.

\section{6}

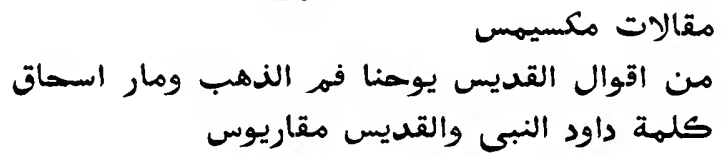




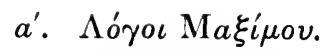

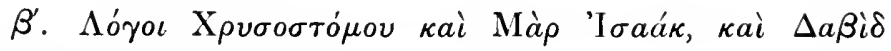
Профиंтov каi Макарі́оv.

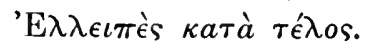

Х $a^{\prime} \rho \tau \eta s^{*} \iota \gamma^{\prime} \times \iota^{\prime} \phi . \rho \xi \zeta^{\prime} \cdot \gamma \rho . \iota a^{\prime}$.

\section{7}

الرسالة التى نزلت من السها في روميـة الكبرى على اياه

البطرك اثاناسيوس التئ نزيت

قصة السيـدة مريـم عند انتسقالها من هذا العالم الى العاله

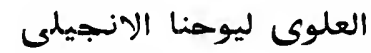

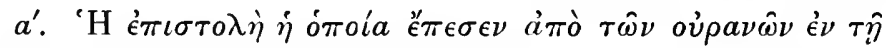

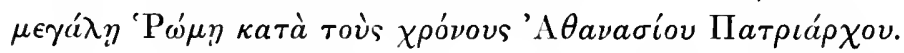

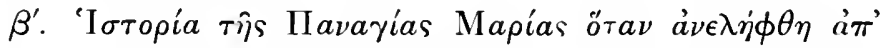

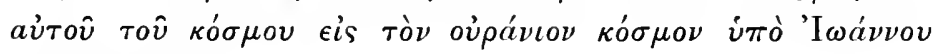

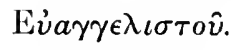

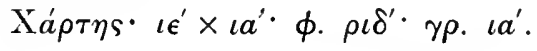

\section{8}

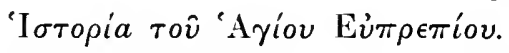

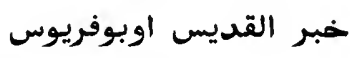

$\mathrm{X} \dot{\alpha} \rho \tau \eta \varsigma^{\cdot} \iota \eta^{\prime} \times \iota \gamma^{\prime} \phi \phi . \nu \beta^{\prime} \cdot \gamma \rho . \iota \delta^{\prime}$.

489

'I $\sigma \tau o p i a$ 'I $\omega \sigma \eta \dot{\phi} \phi$.

قصة يوسف

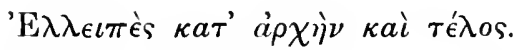

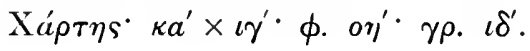

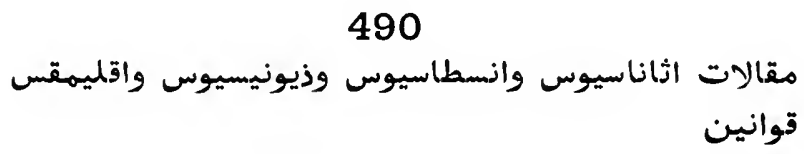

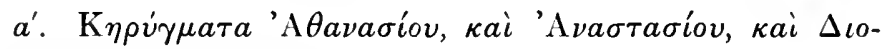
vvбіоч, каі К入інакоs.

$\beta^{\prime}$. Kavóves.

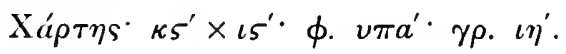




\section{1}

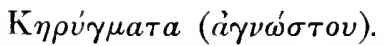

مقالات

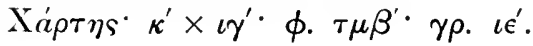

\section{2}

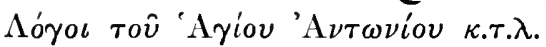

اقوال القديس انطونيوس والخ

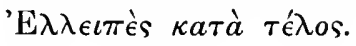

$\mathrm{X} \dot{\alpha} \rho \tau \eta \varsigma^{*} \kappa \varsigma^{\prime} \times \iota \eta^{\prime} \cdot \phi . \sigma o \zeta^{\prime} \cdot \gamma \rho . \kappa^{\prime}$.

\section{3}

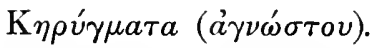

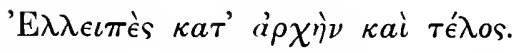

$\mathrm{X} \alpha^{\prime} \rho \tau \eta \varsigma^{\cdot} \kappa \beta^{\prime} \times 1 \delta^{\prime} \cdot \phi . \rho \rho^{\prime} \cdot \gamma \rho .15^{\prime}$.

\section{4}

$$
\text { اقوال معلم وتلميذ }
$$

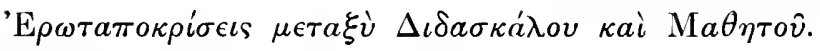
$\mathrm{X} a ́ \rho \tau \eta s^{\cdot} \kappa^{\prime} \times \iota \delta^{\prime} \cdot \phi . v \iota^{\prime} \gamma \rho . \iota \epsilon^{\prime}$.

\section{5}

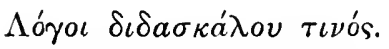

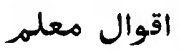

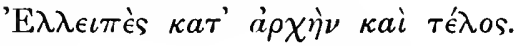

$\mathrm{X} a ́ \rho \tau \eta \varsigma^{*} \iota \theta^{\prime} \times \iota \gamma^{\prime} \gamma \rho . \iota a^{\prime}$.

\section{$+496$}

\section{عظات مارى باسيليوس اسقف قيسلرية}

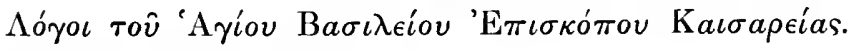

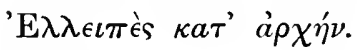

Хá $\rho \tau \eta \varsigma^{\cdot} \kappa^{\prime} \times \iota \gamma^{\prime} \cdot \phi . \rho o a^{\prime} \cdot \gamma \rho . \iota \eta^{\prime}$.

G. A. M. 


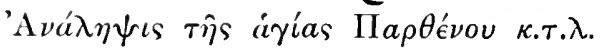

\section{7}

انتقال مرتمريم من الارض والخ

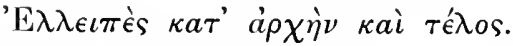

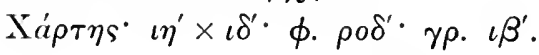

\section{$+498$}

$a^{\prime} . \Sigma v \nu a \xi a ́ p \iota o \nu$.

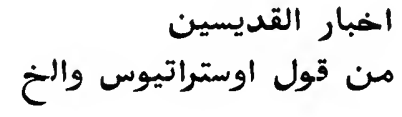

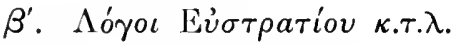

$\mathrm{Xá} \rho \tau \varsigma^{\cdot} \iota \zeta^{\prime} \times \iota \beta^{\prime} \cdot \phi . \sigma \xi \gamma^{\prime} \cdot \gamma \rho . \quad \delta^{\prime}$.

\section{9}

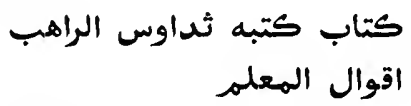

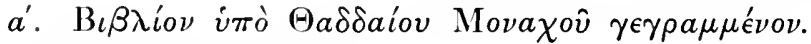

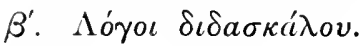

$\mathrm{X} \alpha \rho \tau \eta s^{*} \iota \eta^{\prime} \times \imath \gamma^{\prime} \phi . \sigma \iota^{\prime} \gamma \rho . \iota \delta^{\prime}$.

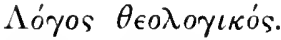

\section{0}

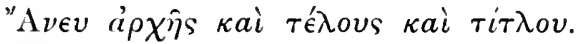

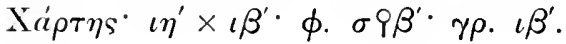

\section{1}

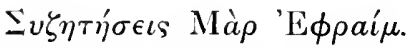

مسمائل القديس افرام

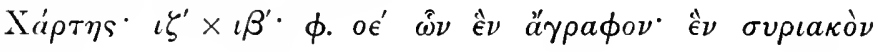
$\phi \hat{u} \lambda \lambda o \nu$ Ė $\tau \hat{\omega} \delta \epsilon \sigma i \mu \omega$.

502

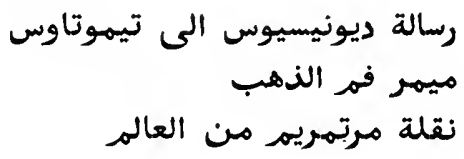




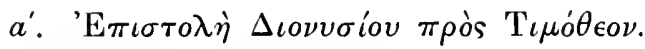

$\beta^{\prime} . \mathrm{K} \eta \dot{\rho} \nu \gamma \mu a \mathrm{X} \rho \nu \sigma o \sigma \tau o ́ \mu o v$.

$\gamma^{\prime}$. 'A

$\mathrm{X} \alpha \rho \tau \eta s^{*} \kappa \epsilon^{\prime} \times \iota \zeta^{\prime} \phi . \rho \iota \gamma^{\prime} \gamma \rho . \iota \eta^{\prime}$.

503

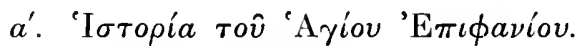

قصة القديس ابيفانيوس

قصة اغريغوريوس الفحسين

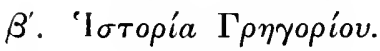

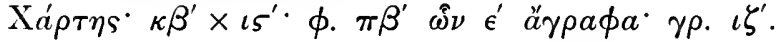

\section{4}

ميامر ثاوذورس ومار افرام ويسطس وابغانيوس اسقف قبرص

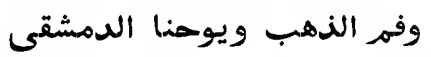

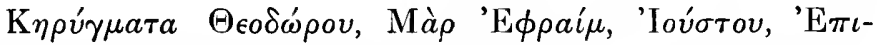

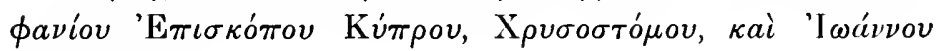
$\Delta a \mu a \sigma \kappa \eta \nu o \hat{v}$.

$\Sigma \eta \tau o \phi a \gamma \eta \tau o ́ \nu$.

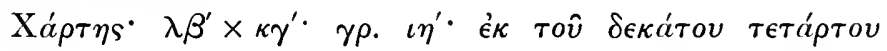

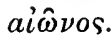

\section{5}

'I $\sigma \tau o p i ́ a \iota \tau \hat{\omega} \nu$ 'A $\gamma i \omega \nu$.

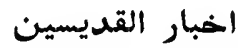

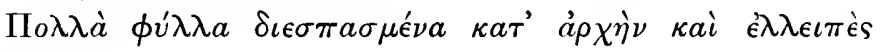

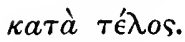

$\mathrm{X} a ́ \rho \tau \eta \varsigma^{\cdot} \kappa \epsilon^{\prime} \times \iota \zeta^{\prime} \phi . \tau \lambda \zeta^{\prime} \gamma \rho . \iota \eta^{\prime}$.

\section{6}

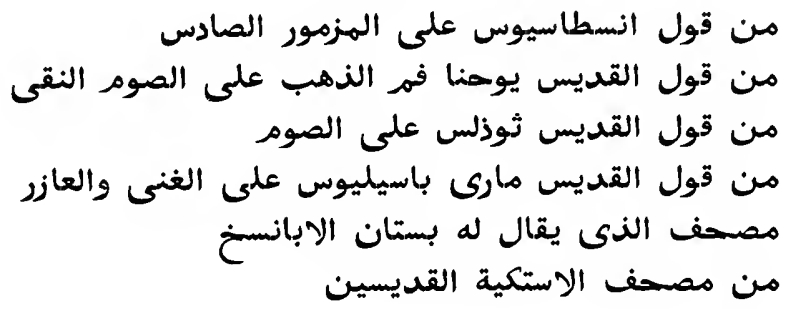

I $3-2$ 


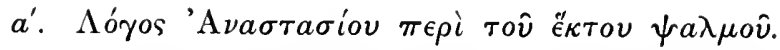

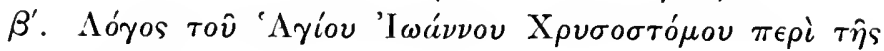

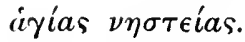

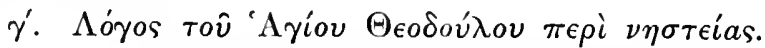

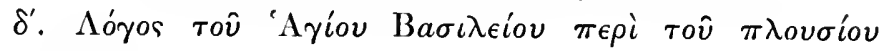

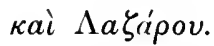

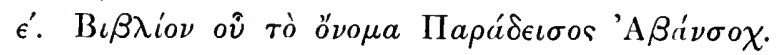

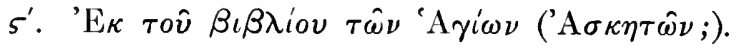

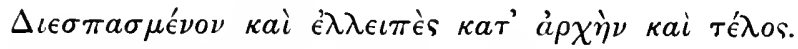

$\mathrm{X} a \rho \tau \eta s^{*} \kappa a^{\prime} \times \iota \delta^{\prime} \cdot \phi \cdot \rho \circ \zeta^{\prime} \gamma \rho . \iota \zeta^{\prime}$.

\section{7}

$a^{\prime} .{ }^{\prime} \mathrm{E} \xi \eta \gamma \eta \dot{ } \sigma \epsilon \iota$.

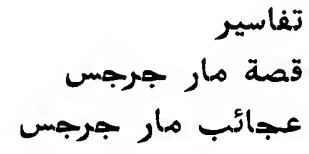

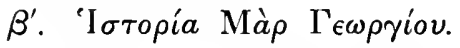

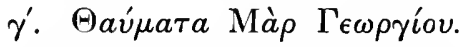

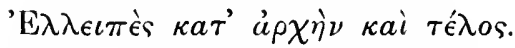

$\mathrm{X} a ́ \rho \tau \eta \varsigma^{\cdot} \kappa a^{\prime} \times \iota \delta^{\prime} \cdot \phi . \rho \xi \gamma^{\prime} \hat{\omega} \nu \gamma^{\prime} a^{\prime} \gamma \rho a \pi \tau a \cdot \gamma \rho . \iota \eta^{\prime}$.

\section{8}

قصة تعرف اقليهس بوالديه واخوته من قبل بطرس الرسول

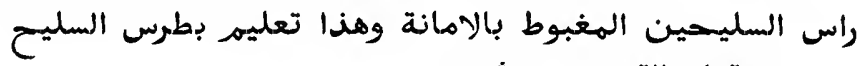

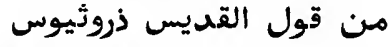

قصص الابهات الرهبان القديسين ورونين

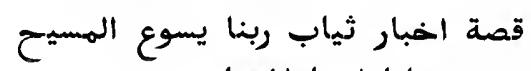

من وصايا ابينا اشعيا تمابيا

من قول مار اسحاق وصات الينا

قصة نفنوثيوس

تذكـرة ما فعل سيسيدنا يسوع الهـئح على عهد بلاطوس

اقوال الابهات 


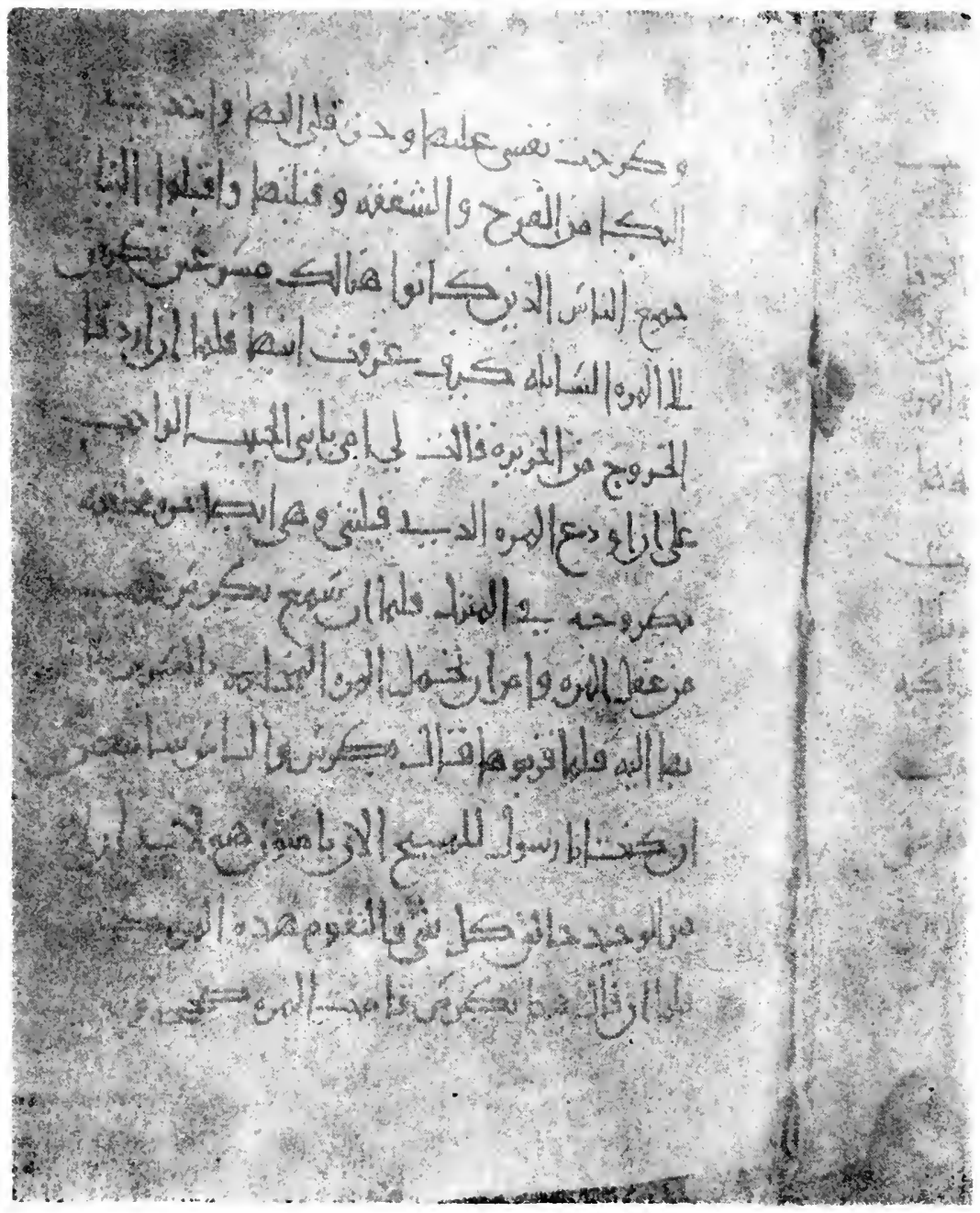




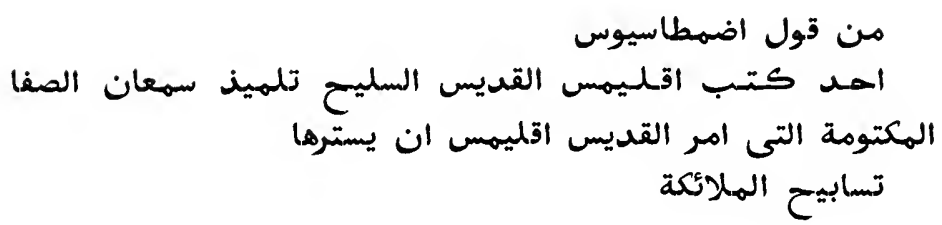

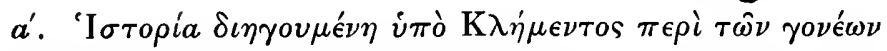

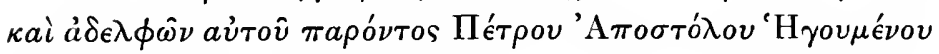

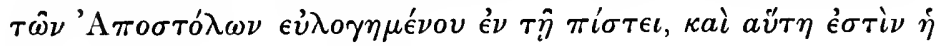

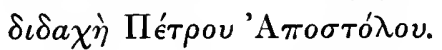

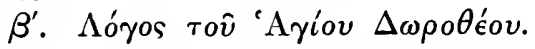

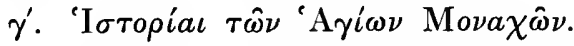

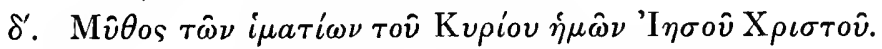

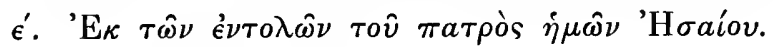

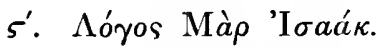

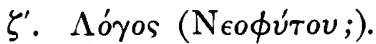

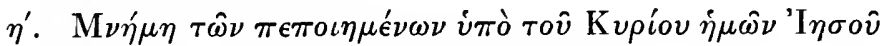

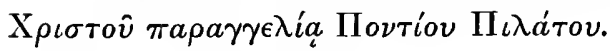

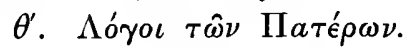

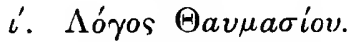

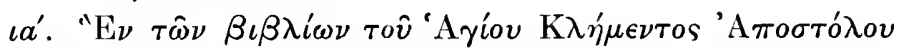

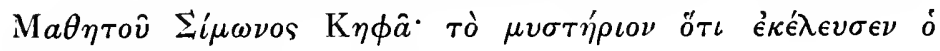

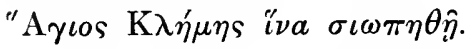

$\iota \beta$ '. ' $\Omega \delta a i \tau \hat{\imath} \nu$ 'A $\gamma \gamma \hat{\epsilon} \bar{\lambda} \omega \nu$.

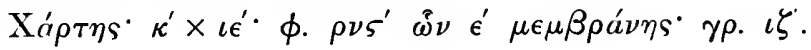

\section{9}

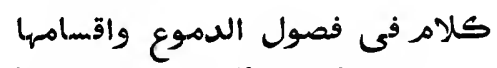

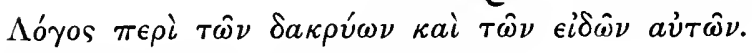

$\mathrm{X} \alpha \dot{\rho} \rho \tau \eta \varsigma^{\cdot} \kappa \beta^{\prime} \times \iota \epsilon^{\prime} \cdot \phi . \sigma \pi \gamma^{\prime} \gamma \rho . \iota \eta^{\prime}$.

\section{0}

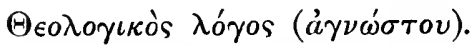

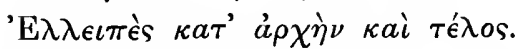

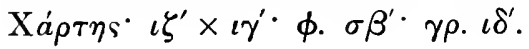




\section{1}

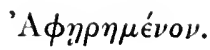

\section{2}

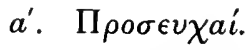

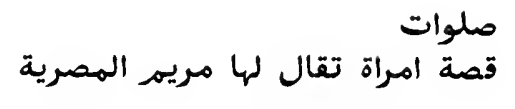

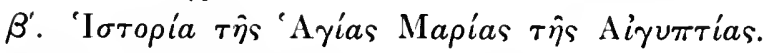

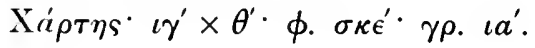

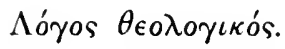

\section{$+513$}

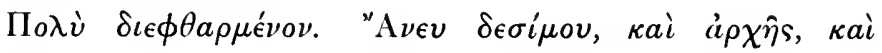

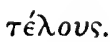

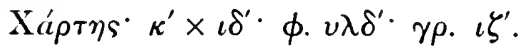

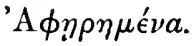

\section{4 \\ $515\}$}

\section{$+516$}

'I $\sigma \tau o \rho i ́ a \delta$

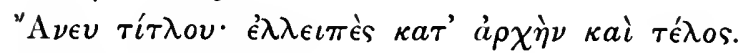

$\mathrm{M}_{\epsilon} \mu \beta \rho \alpha \dot{\nu} \eta \eta^{\cdot} \kappa \beta^{\prime} \times \iota \epsilon^{\prime} \cdot \phi .9 \beta^{\prime} \cdot \gamma \rho . \iota \zeta^{\prime}$.

'A $\phi \eta \rho \eta \mu e ́ v a$.

\section{7 \\ 518 \\ 519}

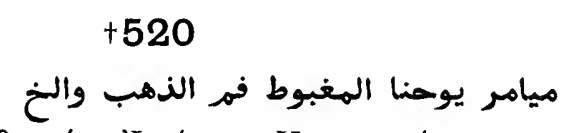

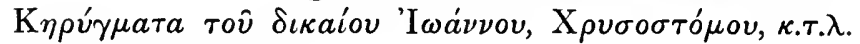

$\Pi о \lambda \dot{v} \delta \iota \epsilon \phi \theta a \rho \mu \mu^{\prime} \nu o \nu$.

$\mathrm{X} a \dot{\rho} \tau \eta \varsigma^{\cdot} \iota \eta^{\prime} \times \iota \delta^{\prime} \cdot \phi . \sigma 9 a^{\prime} \cdot \gamma \rho . \iota \delta^{\prime}$. 


\section{1}

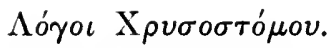

اقوال فم الذهب

$\Pi о \lambda \dot{v} \delta \iota € \phi \theta a \rho \mu \epsilon^{\prime} \nu o \nu$.

$\mathrm{X} a ́ \rho \tau \eta \varsigma^{\bullet} \iota \theta^{\prime} \times \iota \delta^{\prime} \cdot \phi . \rho \mu \eta^{\prime} \cdot \gamma \rho . \iota \epsilon^{\prime}$.

\section{2}

قصة الطاهر الهختار مار الياس الغيور البار الصاعد الى السها

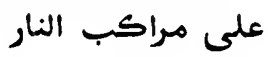
شفايمّه

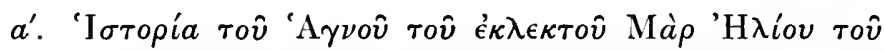

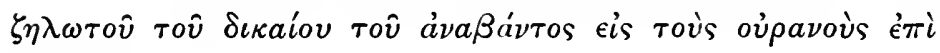
$\dot{a} \mu a \xi \hat{\omega} \nu \pi v \rho o ́ s$.

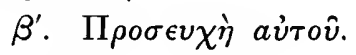

$\mathrm{X} a ́ \rho \tau \eta \varsigma^{*} \iota \eta^{\prime} \times \iota \gamma^{\prime} \cdot \phi . \rho \mu \delta^{\prime} \cdot \gamma \rho . \iota \gamma^{\prime}$.

\section{3}
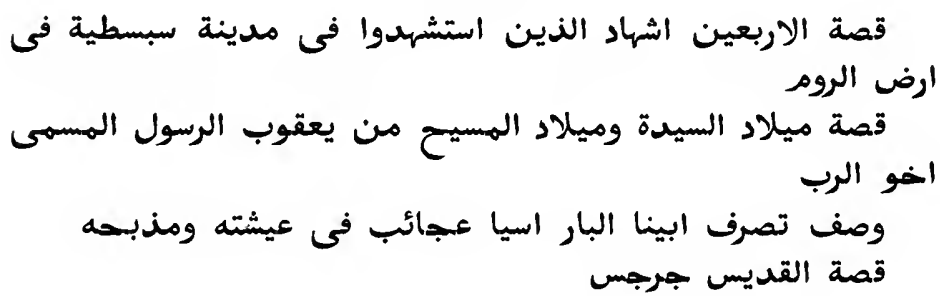

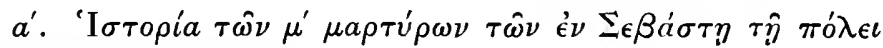

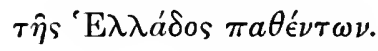

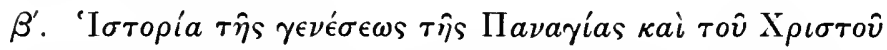

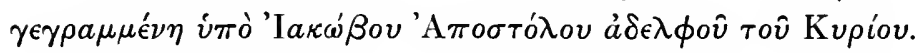

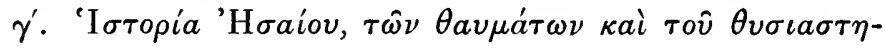
píov aủंôิ.

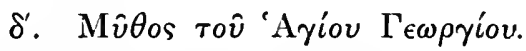

$\mathrm{X} a ́ \rho \tau \eta s^{\prime} \iota \epsilon^{\prime} \times \iota a^{\prime} \cdot \phi . \rho \mu \beta^{\prime} \cdot \gamma \rho . \iota^{\prime}$.

\section{4}

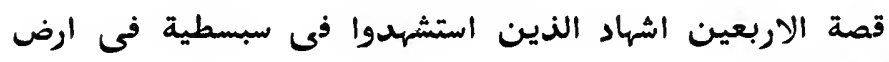




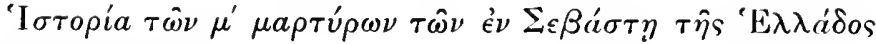
$\pi a \theta \epsilon ́ \nu \tau \omega \nu \kappa . \tau . \lambda$.

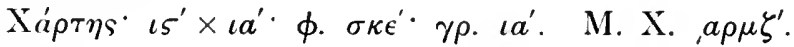

\section{5}

$\mathrm{K} \eta \rho v ́ \gamma \mu a \tau a \mathrm{M} \dot{\alpha} \rho \mathrm{K} a \sigma \sigma \iota a \nu o \hat{.}$.

$$
\text { مقالات مار كاسانيوس }
$$

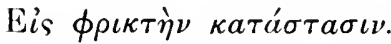

$\mathrm{X} a \rho \tau \eta \varsigma^{\cdot} \kappa a^{\prime} \times \iota \delta^{\prime} \cdot \phi . \sigma \lambda \zeta^{\prime} \gamma \rho . \iota \varsigma^{\prime}$.

\section{6}

'I $\sigma \tau о \rho i ́ a ~ \tau \hat{\eta} \varsigma$ 'E $\kappa \kappa \lambda \eta \sigma i a s$.

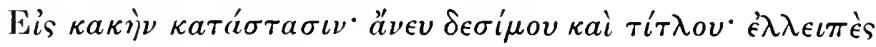

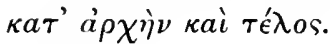

$\mathrm{X} \alpha \rho \tau \eta \varsigma^{\circ} \kappa \varsigma^{\prime} \times \iota \varsigma^{\prime} \phi . \rho \lambda \varsigma^{\prime} \gamma \rho . \kappa \gamma^{\prime}$.

\section{7}

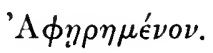

\section{8}

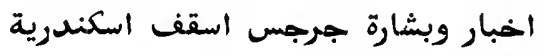

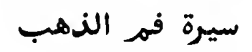

الهنظر الذى راه الذه الذهب اشيشيوس ليهنا الهغبوط

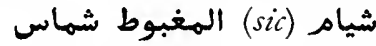

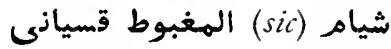

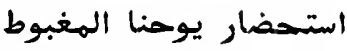

كتاب الملك الى البـطرك ...... الهـــبـوط الهغوط من ارقاذيـوس

وهنوريوس

خبر الارملة الاسكندرانية من اشخاص ثأوفيلس

حرمة ثاوفنسطس وكرمها

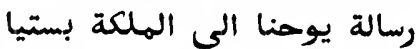

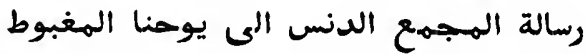

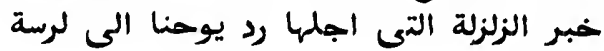




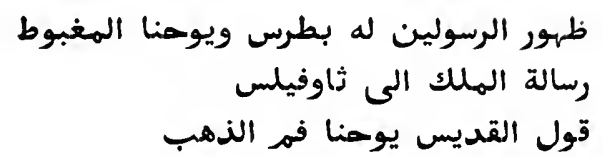

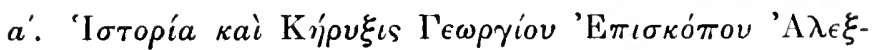

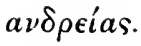

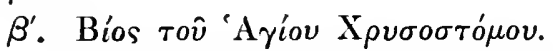

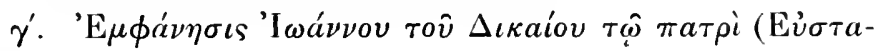
$\theta i \omega ;)$.

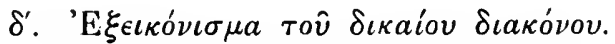

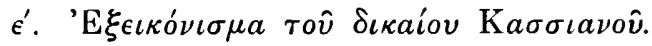

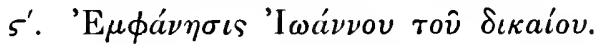

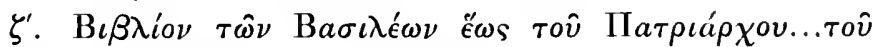

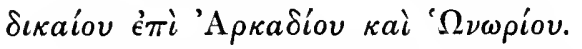

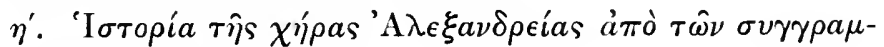
$\mu a ́ \tau \omega \nu \Theta \epsilon o \phi i ́ \lambda o v$.

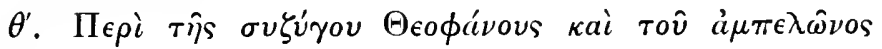

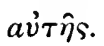

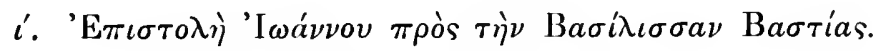

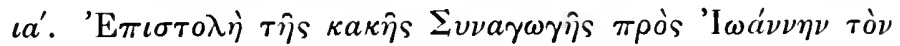
$\Delta і \kappa a \iota \nu^{\prime}$

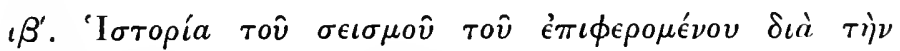

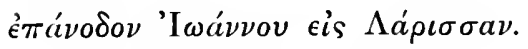

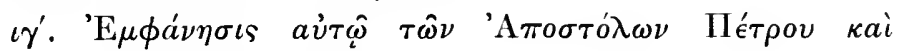

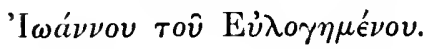

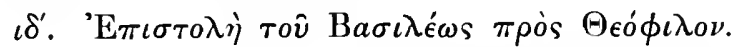

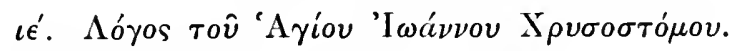

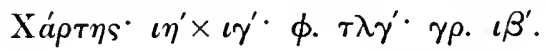

529

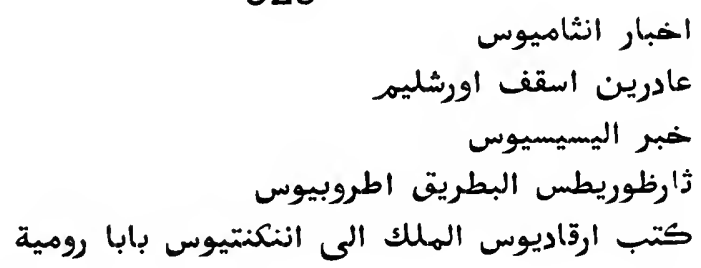

G. A. M. 


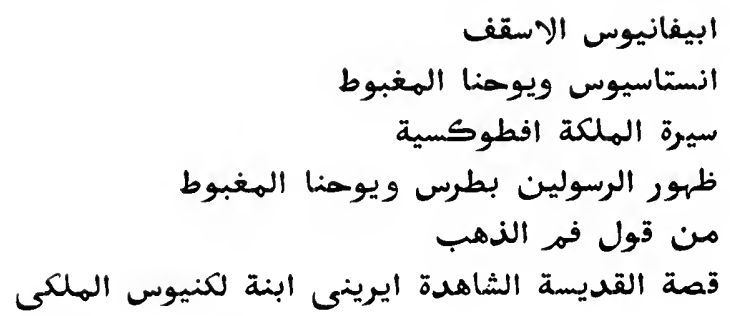

$a^{\prime}$. 'I $\sigma \tau o \rho i ́ a$ 'A $\nu \theta i \mu o v$.

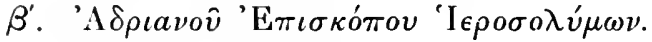

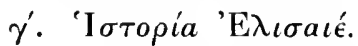

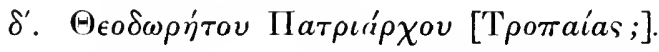

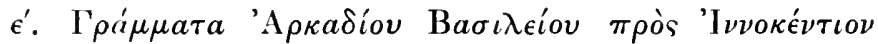
П $a \pi \hat{a} \nu$ 'Р $\omega \dot{\mu} \eta$ s.

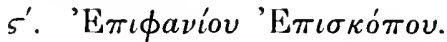

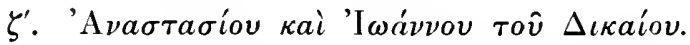

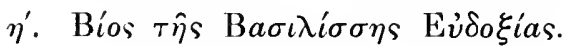

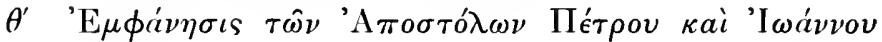

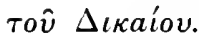

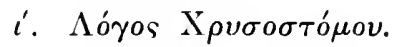

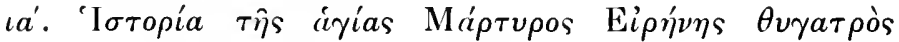

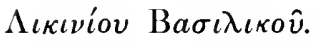

$\mathrm{Х} a \rho \tau \eta \varsigma^{\cdot} \kappa a^{\prime} \times \iota \delta^{\prime} \cdot \phi . \tau \kappa \zeta^{\prime} \gamma \rho . \iota \delta^{\prime}$.

\section{0}

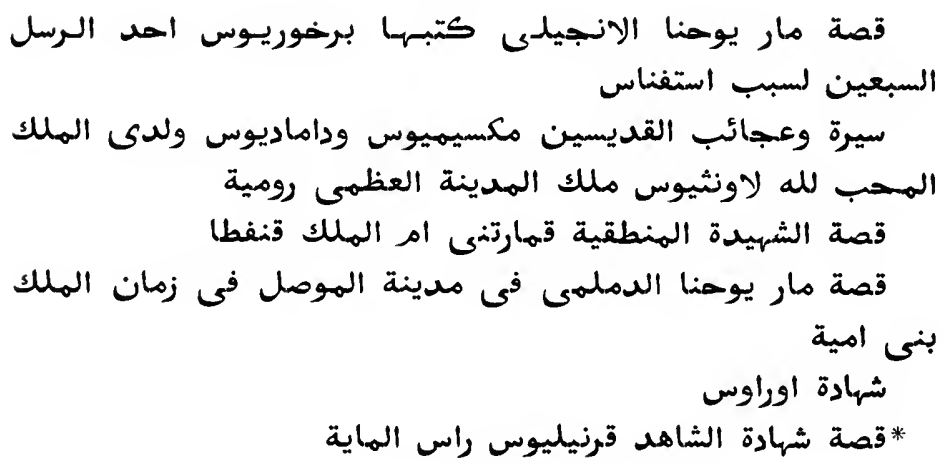




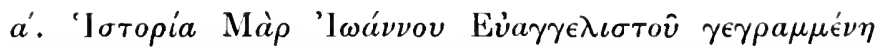

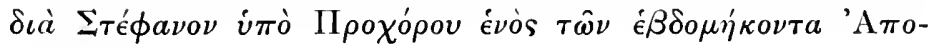
$\sigma \tau o ́ \lambda \omega \nu$.

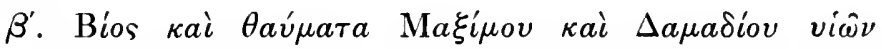

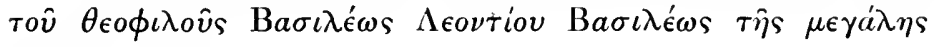
$\pi o ́ \lambda \epsilon \omega s$ 'Р $\omega^{\prime} \mu \eta \varsigma$.

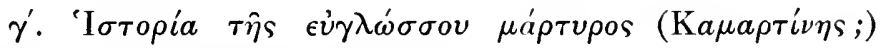
$\mu \eta \tau \rho o ̀ s ~ \tau o \hat{v ~ B a \sigma \iota \lambda \epsilon ́ \omega s ~(K a v a ́ \delta \chi ;) . ~}$

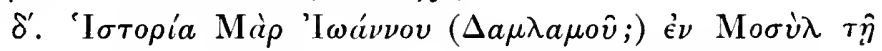

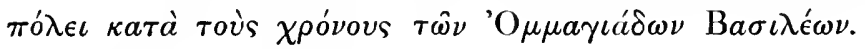

є'. 'I

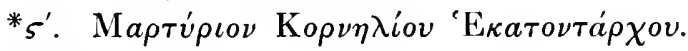

$\mathrm{X} a ́ \rho \tau \eta \varsigma^{\cdot} \iota \zeta^{\prime} \times \iota \gamma^{\prime} \cdot \phi . v \iota s^{\prime} \cdot \gamma \rho . \iota \gamma^{\prime}$.

\section{1}

$$
\text { قصة اوفمانوس بولص الرسول }
$$

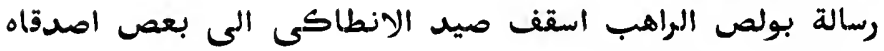

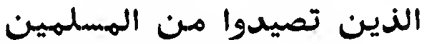

خبـر وجمود الصليب الهعظمه علدي يلهين يدى هلانة اهر الهلك
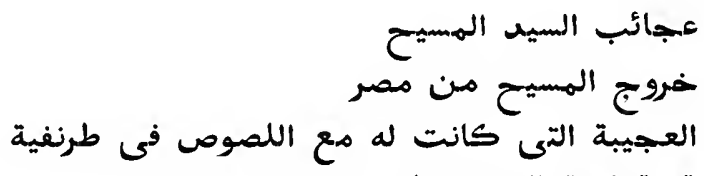

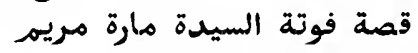

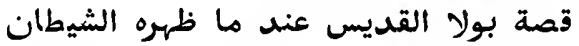

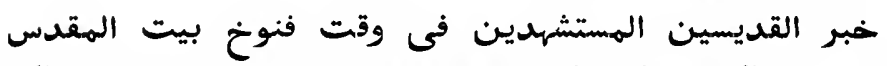
فى ايام مهلكة هرقك على الروم وبولى الهينى بشروان كسرى فمى مهلكة

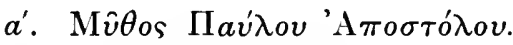

من قول ابينا انسظرط

$\beta^{\prime}$. 'I $\sigma \tau$ ropía Ev̉uevíov.

اطروباريات

$$
14-2
$$




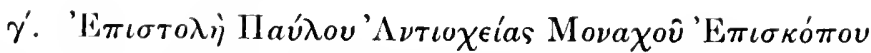

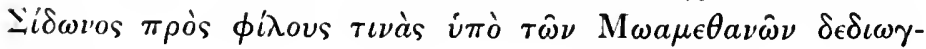
$\mu$ évous.

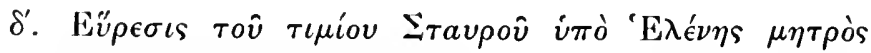

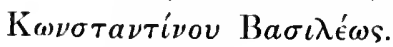

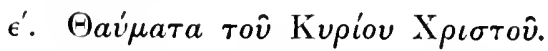

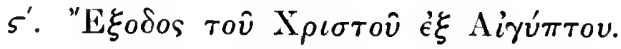

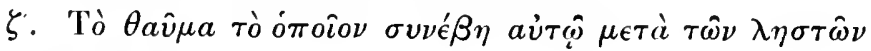

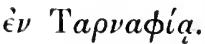

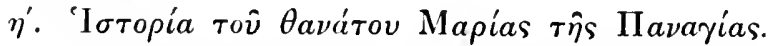

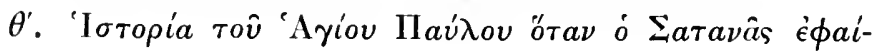
$\nu \in \tau o$ à่ $\hat{\omega}$.

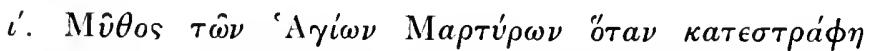
'Iє

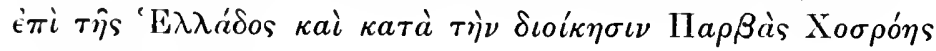

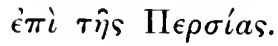

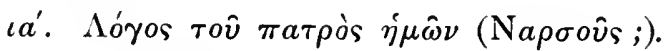

$\iota \beta^{\prime}$. Трота́рца.

Ха́ $\rho \tau \eta \varsigma^{\cdot} \iota \epsilon^{\prime} \times \iota a^{\prime} \cdot \phi . \tau \xi \beta^{\prime} \cdot \gamma \rho . \iota \beta^{\prime}$.

\section{2}

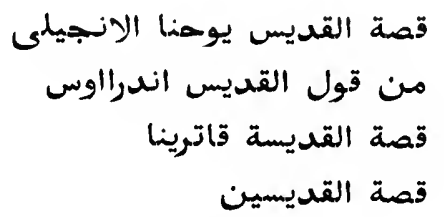

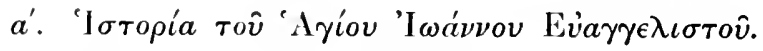

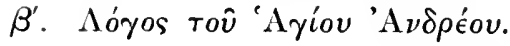

$\gamma$ '. 'I

$\delta^{\prime}$. 'I $\sigma \tau o \rho i ́ a ~ \tau \hat{\omega} \nu$ 'A $\boldsymbol{\gamma} i \omega \nu$.

Х $\iota \dot{\rho} \tau \eta \varsigma^{\cdot} \iota \zeta^{\prime} \times \iota \beta^{\prime} \cdot \phi . \sigma \beta^{\prime} \cdot \gamma \rho . \iota \beta^{\prime}$. 


\section{3}

قصة القديس نيلص

من قول القديس افرام : من قول يوحنا الدمشكى فيسى

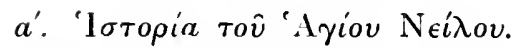

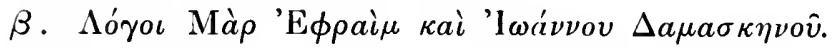

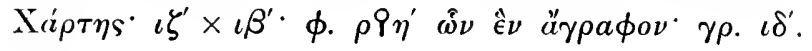

534

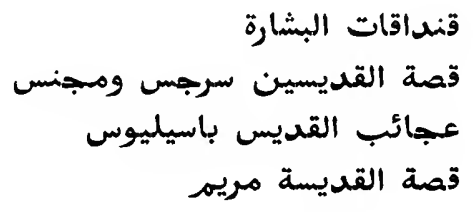

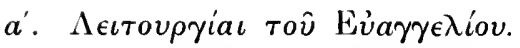

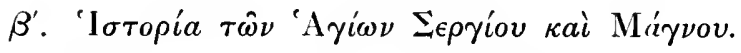

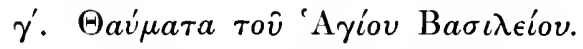

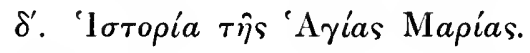

$\mathrm{X} a ́ \rho \tau \eta \varsigma^{\cdot} \iota \zeta^{\prime} \times \iota \gamma^{\prime} \phi . \tau \mu \varsigma^{\prime} \gamma \rho . \iota \delta^{\prime}$.

\section{5}

ميامر يوحنا الدمشكى واكسانيوس وبطرس بابا وبطرس بطرك الا سكندرية

قصة القديس ارميا الحارث واصدابه الذين استشهدوا بخبران

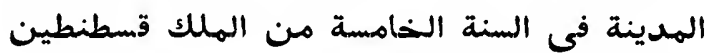

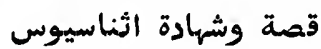

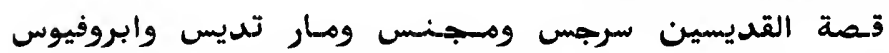

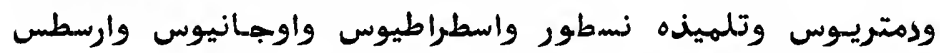

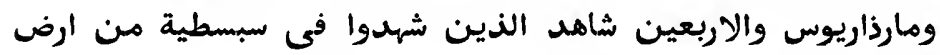

$$
\begin{aligned}
& \text { مار اسيط'ث ابكليذس الناسك وسيرته الهمجبة }
\end{aligned}
$$

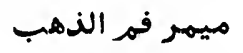

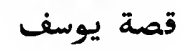




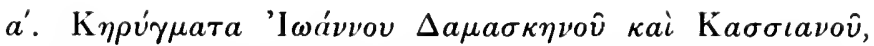

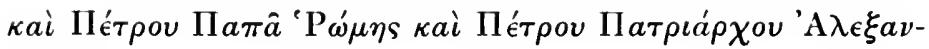
Speías.

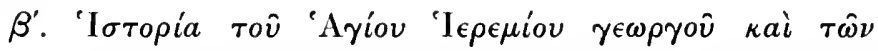

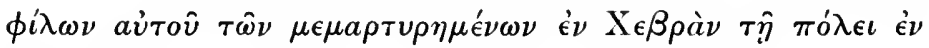

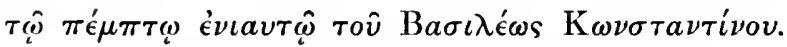

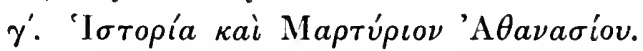

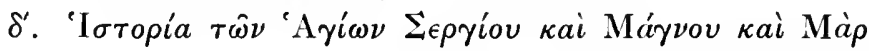

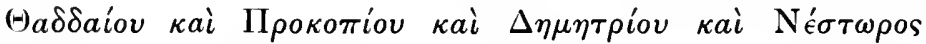

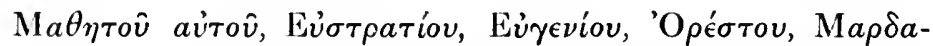

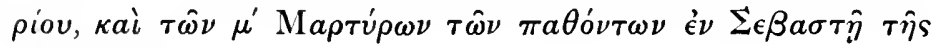
'E $\mathrm{\lambda} \lambda a ́ \delta o s$.

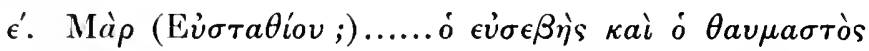
Bios aùtov.

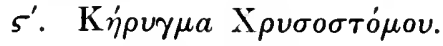

$\zeta$ '. 'І $\sigma \tau \tau o \rho i ́ a$ ' $\mathrm{I} \omega \sigma \eta \dot{\phi} \phi$.

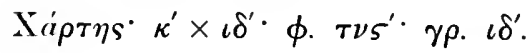

\section{6}

كلام واخبار الابا القديسين من انطونيوس واثانسيوس والخ

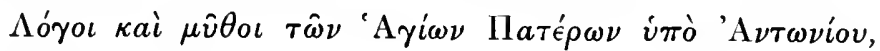

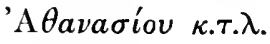

Хá $\rho \tau \eta s^{\cdot} \kappa a^{\prime} \times \iota \gamma^{\prime} \cdot \phi . \tau o \beta^{\prime} \cdot \gamma \rho . \iota \gamma^{\prime}$.

\section{7}

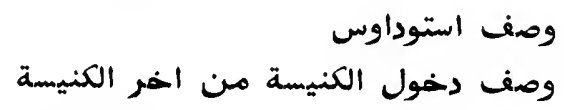

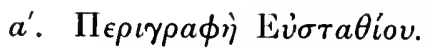

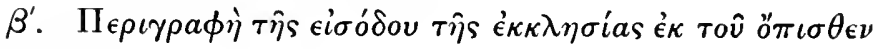

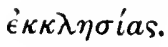

$\mathrm{X}^{\prime} \rho \tau \eta \varsigma^{\circ} \kappa^{\prime} \times \iota \delta^{\prime} \cdot \phi . \sigma \theta^{\prime} \cdot \gamma \rho . \iota^{\prime}$. 


\section{8}

$$
\text { مقالات فم الذهب والخ }
$$

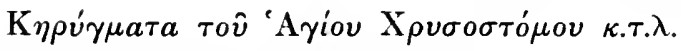

$\mathrm{X} \alpha \rho \rho \eta \varsigma^{*} \kappa \epsilon^{\prime} \times \iota \zeta^{\prime} \phi . v o \zeta^{\prime} \gamma \rho . \kappa^{\prime}$.

\section{9}

$$
\begin{aligned}
& \text { قصة الطوبانى مار بطروس }
\end{aligned}
$$

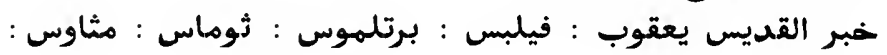

$$
\begin{aligned}
& \text { بنيمن : لوقا }
\end{aligned}
$$

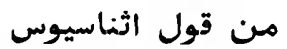

$$
\begin{aligned}
& \text { [قصة...بطرس ريس التلاميذ وبشارته }
\end{aligned}
$$

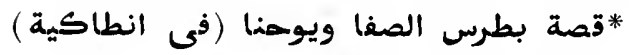

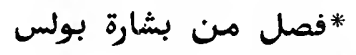

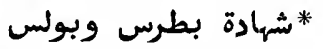

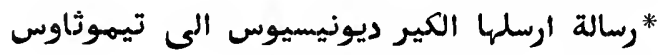

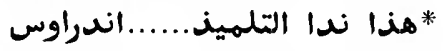

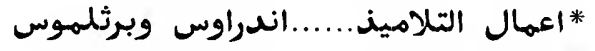

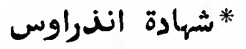

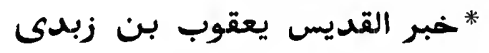

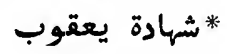

$$
\begin{aligned}
& \text { قصة يوحنا بن زبدى :قورة كروز الانجيل }
\end{aligned}
$$

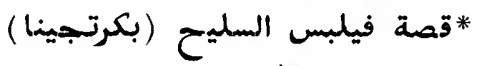

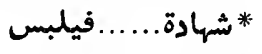

$$
\begin{aligned}
& \text { *شارة برثلمهوس و اكرازه في مدينة الوأح }
\end{aligned}
$$

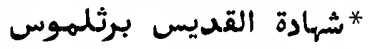

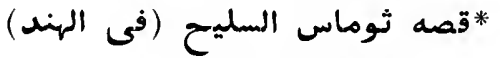

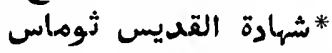

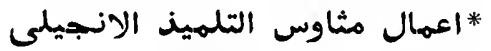

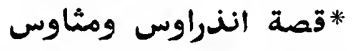

$$
\begin{aligned}
& \text { شهادة القديس مثى الانسجيلى ومنس }
\end{aligned}
$$




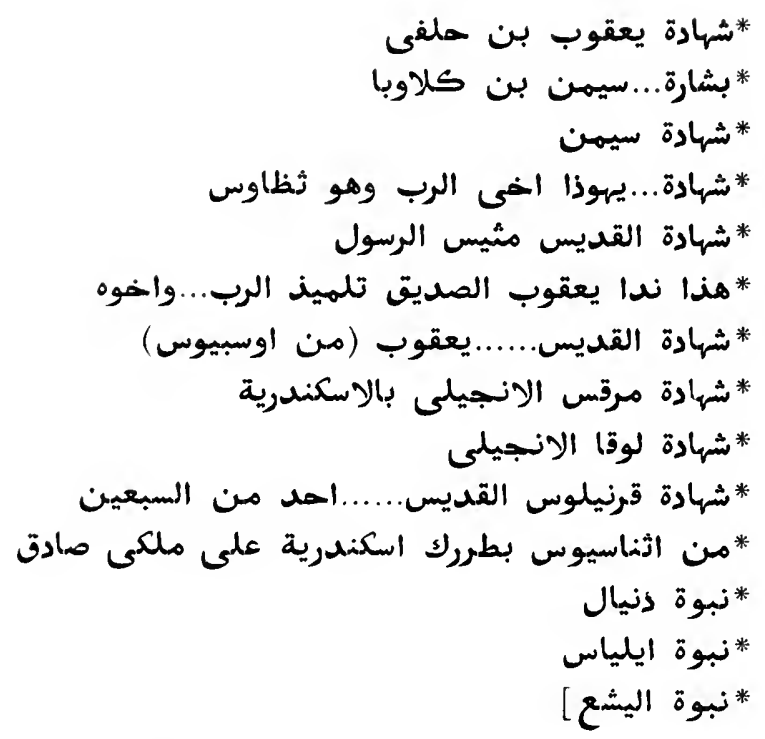

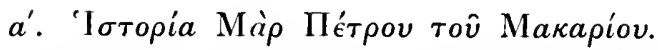

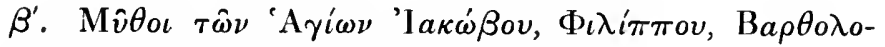

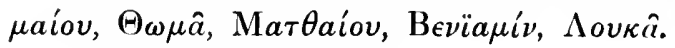

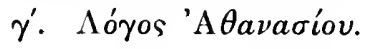

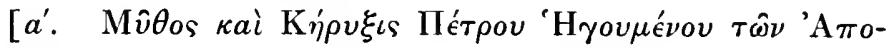
$\sigma \tau o ́ \lambda \omega \nu$.

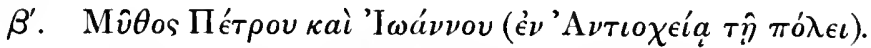

$\gamma$ '. 'А

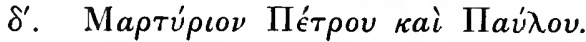

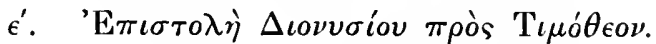

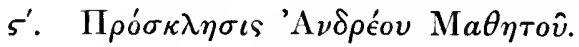

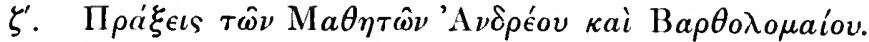

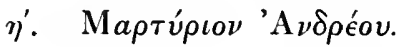

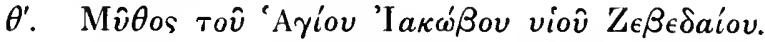

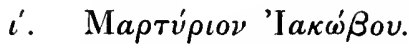

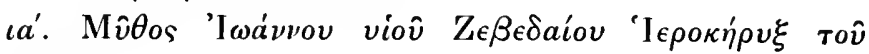

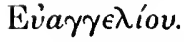

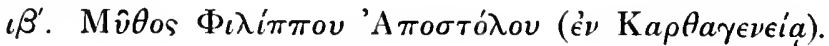




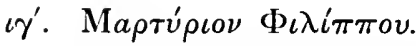
$\gamma u ́ \pi \tau o v$.

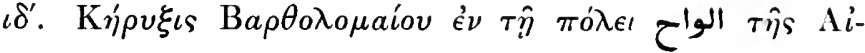

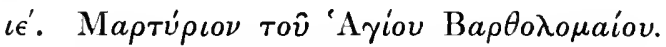

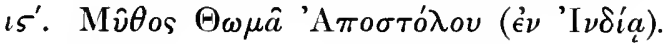

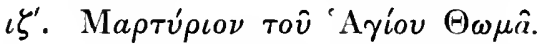

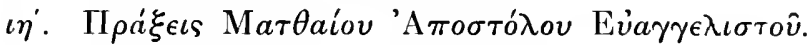

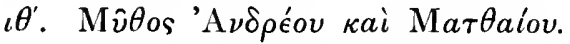

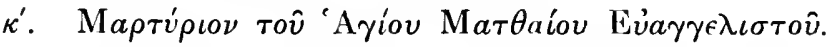

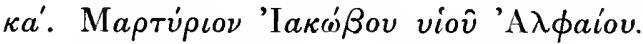

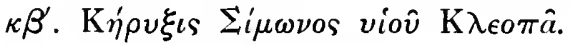

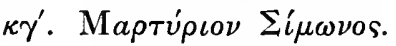

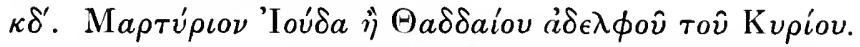

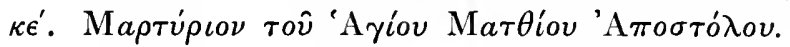

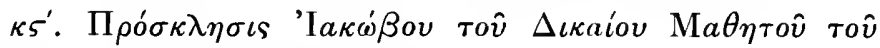

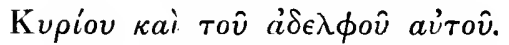

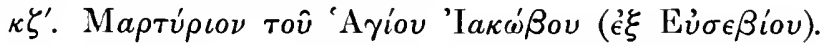

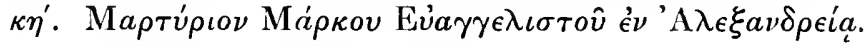

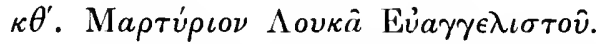

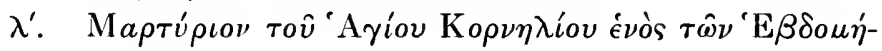

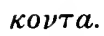

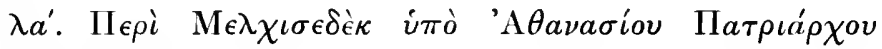
'A $\lambda \epsilon \xi a \nu \delta \rho \epsilon i a s$.

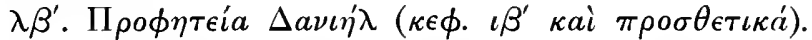

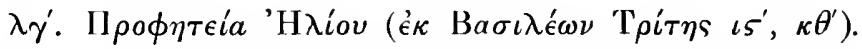

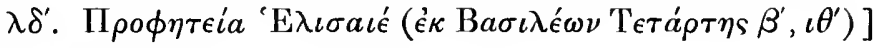

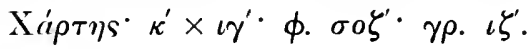

\section{0}

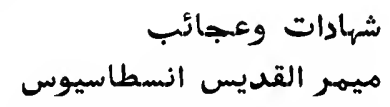

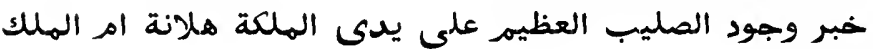

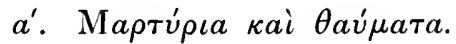

G. A. M. 


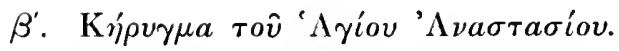

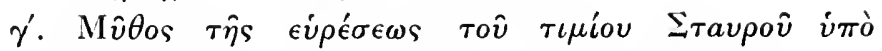

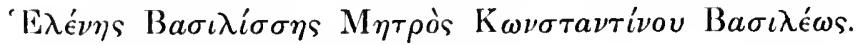

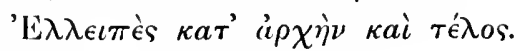

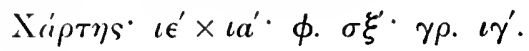

\section{1}

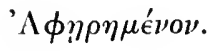

\section{2}

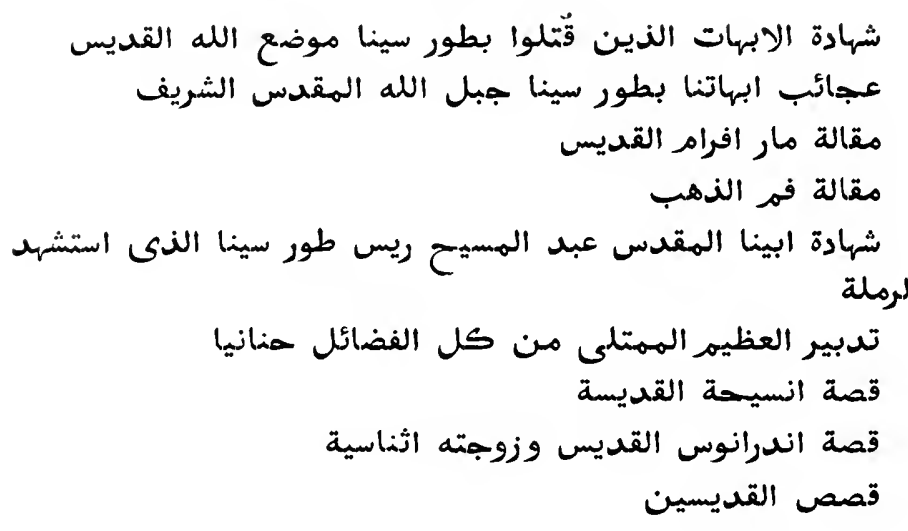

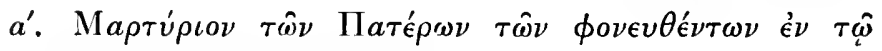

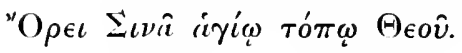

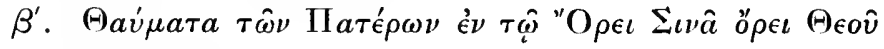

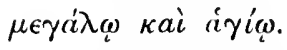

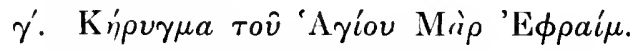

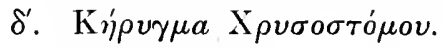

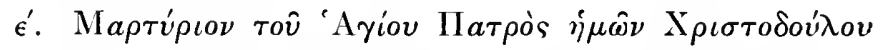

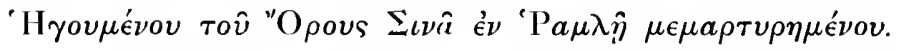

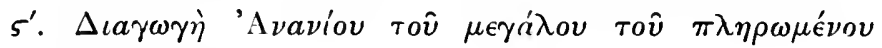

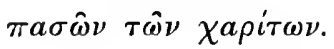

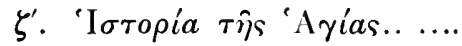

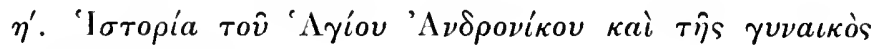

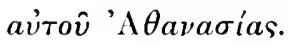


$\theta$ '. 'I $\sigma \tau o \rho i ́ a \iota \tau \hat{\omega} \nu$ 'A $\gamma i \omega \nu$.

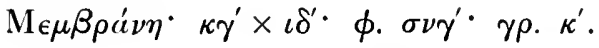

543

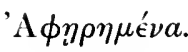

$544\}$

\section{5}

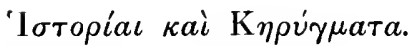

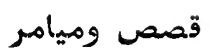

$\mathrm{X}^{\prime} \dot{\rho} \rho \tau \eta \varsigma^{*} \iota \eta^{\prime} \times \iota \delta^{\prime} \cdot \phi . \quad v \lambda \varsigma^{\prime} \cdot \gamma \rho . \iota \delta^{\prime}$.

\section{6}

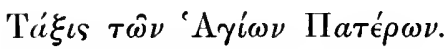

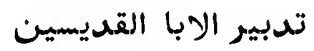

Ха́ $\rho \tau \eta \varsigma^{\cdot} \kappa \epsilon^{\prime} \times \iota \varsigma^{\prime} \cdot \phi . \sigma \mu a^{\prime} \cdot \gamma \rho . \iota \zeta^{\prime}$.

546 bis

اقوال القديسين اغاتون ونفيوثيوس ونبى ايلـوا ويـارقوس ويوحنا القصير والخ القدين

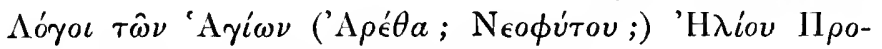

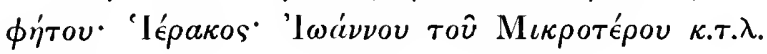

$\mathrm{X} \alpha \dot{\rho} \rho \eta \varsigma^{\cdot} \kappa \epsilon^{\prime} \times \iota \zeta^{\prime} \cdot \phi . \sigma \lambda \eta^{\prime} \cdot \gamma \rho . \iota \zeta^{\prime}$.

\section{7}

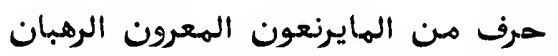

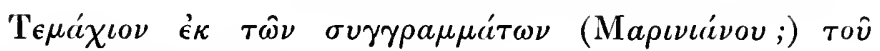

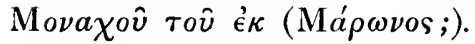

$\mathrm{X} a ́ \rho \tau \eta \varsigma^{\cdot} \kappa \epsilon^{\prime} \times \iota \zeta^{\prime} \cdot \phi . \rho \epsilon^{\prime} \cdot \gamma \rho . \iota \theta^{\prime}$.

\section{8}

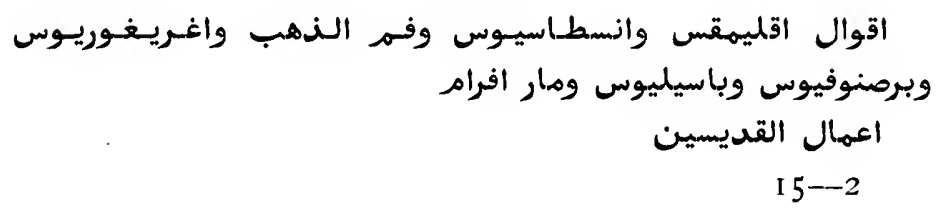




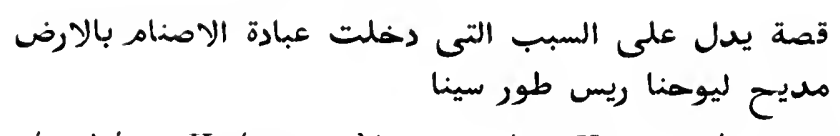

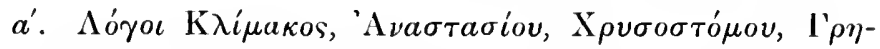

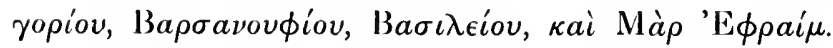

$\beta^{\prime}$. Ilpa' $\xi_{\epsilon \iota s} \tau \hat{\omega} \nu$ 'A $\gamma^{\prime} \omega \nu$.

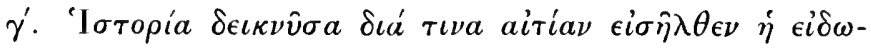

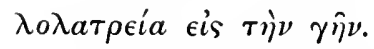

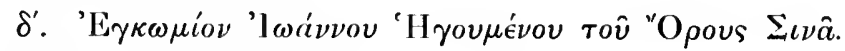

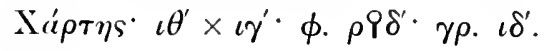

\section{9}

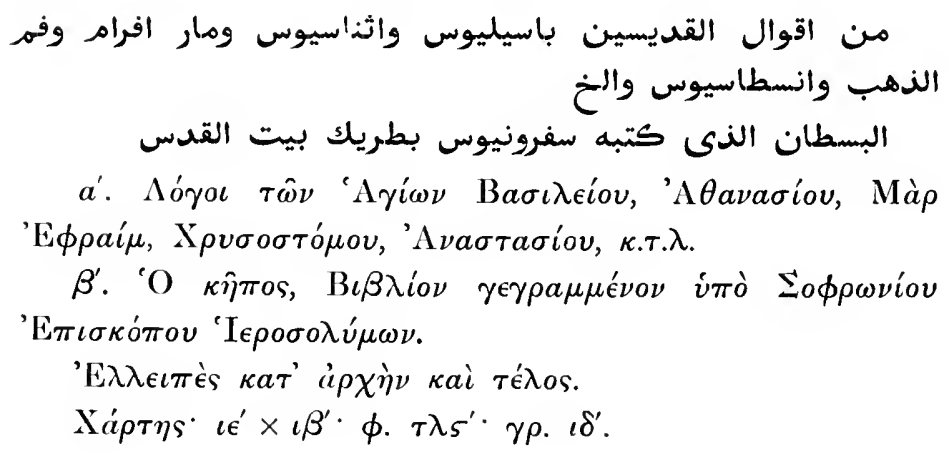

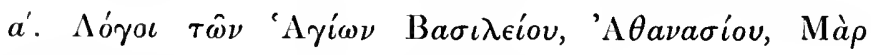

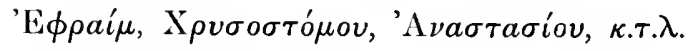

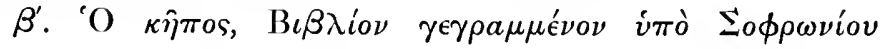

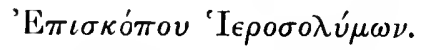

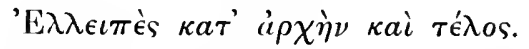

X'́a $\rho \eta \varsigma^{\prime} \iota \epsilon^{\prime} \times \iota \beta^{\prime} \cdot \phi . \tau \lambda \varsigma^{\prime} \cdot \gamma \rho . \iota \delta^{\prime}$.

\section{0}

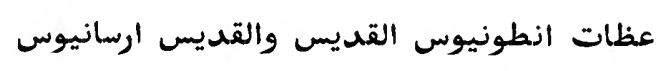

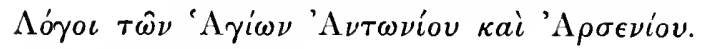

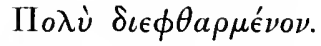

$\mathrm{X} a ́ \rho \tau \eta \varsigma^{\cdot} \iota \zeta^{\prime} \times \imath \beta^{\prime} \cdot \phi . \sigma \pi a^{\prime} \gamma \rho . \imath \eta^{\prime}$.

\section{1}

سيرة القديس ثاوذرس والخ

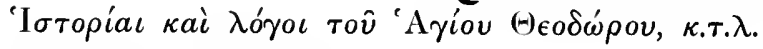

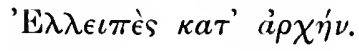

$\mathrm{X} a ́ \rho \tau \eta \varsigma^{\cdot} \iota \varsigma^{\prime} \times \imath \beta^{\prime} \cdot \phi . \rho \mu a^{\prime} \cdot \gamma \rho . \iota \beta^{\prime}$. 


\section{2}

$a$ '. 'I $\sigma \tau o p i a \iota \tau \hat{\omega} \nu$ 'A A

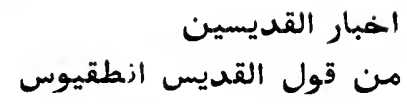

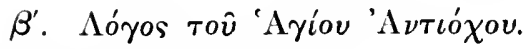

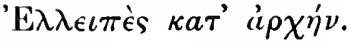

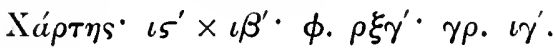

\section{3}

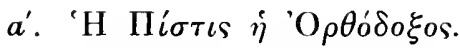

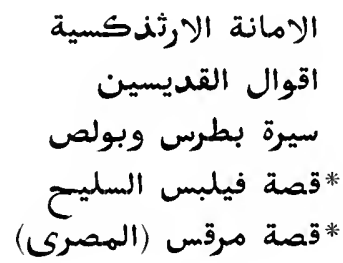

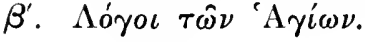

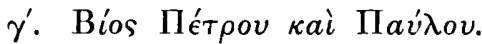

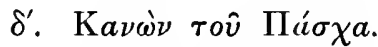

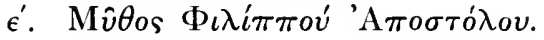

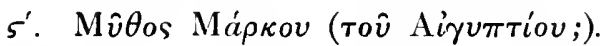

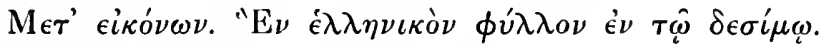

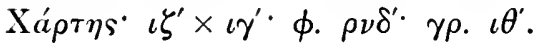

\section{4}

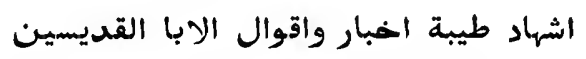

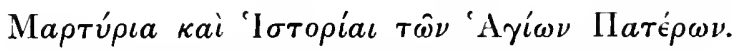

$\mathrm{X} \dot{a} \rho \tau \eta \varsigma^{\cdot} \iota \gamma^{\prime} \times \theta^{\prime} \cdot \phi . \quad \tau \eta^{\prime} \hat{\omega} \nu \boldsymbol{5}^{\prime} \dot{\epsilon} \pi \epsilon \nu \theta \epsilon \tau \iota \kappa a^{\prime} \gamma \rho . \iota a^{\prime}$.

\section{5}

ماية راس وضعها القديس يوحنا الراهب العظيم الهشهور لرهبان الهin

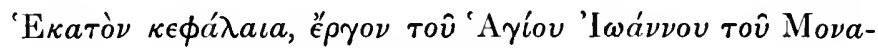

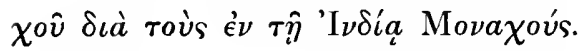

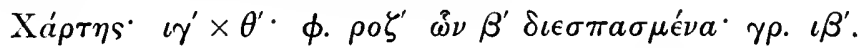




\section{6}

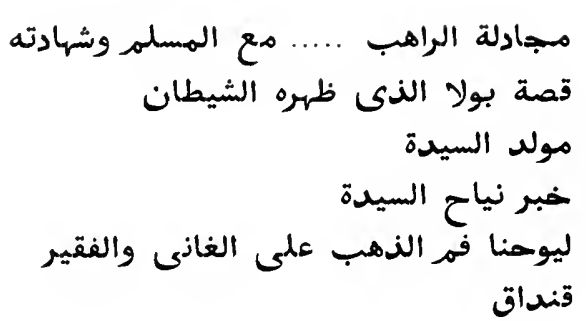

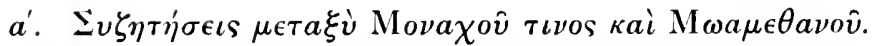

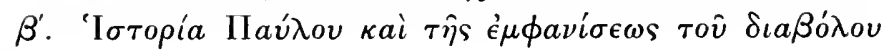
$a \dot{v} \tau \hat{\omega}$.

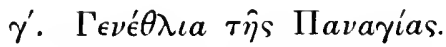

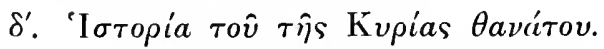

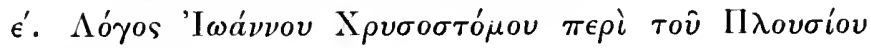
каi $\tau о \hat{v} \Pi \tau \omega \chi o \hat{v}$.

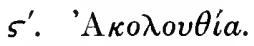

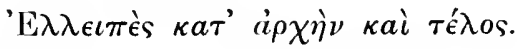

$\mathrm{X} a ́ \rho \tau \eta \varsigma^{\cdot} \iota \epsilon^{\prime} \times \iota^{\prime} \phi . \rho \pi \delta^{\prime} \cdot \gamma \rho . \iota \epsilon^{\prime}$.

$+557$

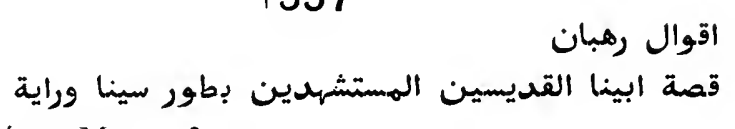

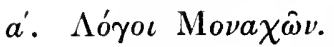

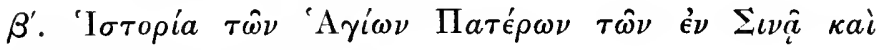

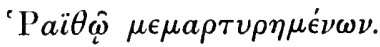

Ха́ $\rho \tau \eta s^{\circ} \iota \eta^{\prime} \times \iota \beta^{\prime} \phi . \tau \iota \gamma^{\prime} \gamma \rho . \iota \beta^{\prime}$.

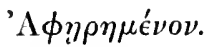

\section{8}

\section{9}

$$
\text { ميامر مار افرام وفم الذهب وباسيليوس والخ }
$$

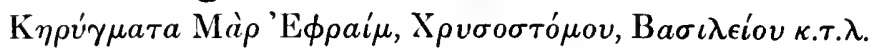

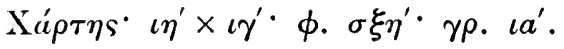




\section{0}

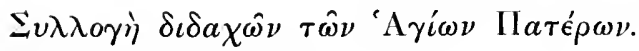

$$
\text { مجمع من تعاليم الابا القديسين }
$$

$\mathrm{X} \alpha \dot{\rho} \rho \eta \varsigma^{\circ} \kappa a^{\prime} \times \iota \delta^{\prime} \cdot \phi . \sigma \xi \gamma^{\prime} \cdot \gamma \rho . \iota \varsigma^{\prime}$.

\section{1}

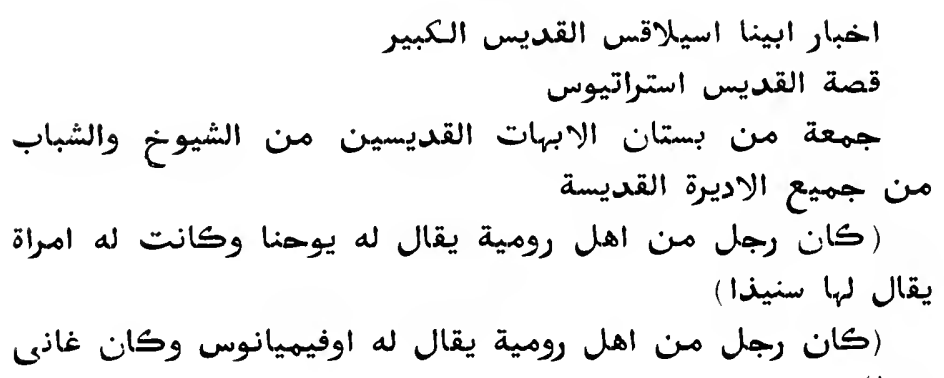

$$
\begin{aligned}
& \text { بستان رهبان طور سينا مها وضع انسطاسيوس } \\
& \text { اقوال فم الذهب } \\
& \text { بعض عجائب ابهات بطول بهور سينا } \\
& \text { عظه ابينا اشعيا } \\
& \text { من قول القديس ثاوذورس القعيا }
\end{aligned}
$$

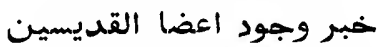

$$
\begin{aligned}
& \text { اقوال اغريغوريوس والخ اعضان القديسن }
\end{aligned}
$$

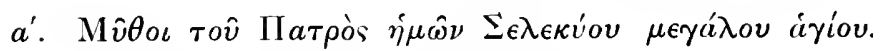

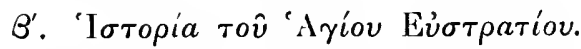

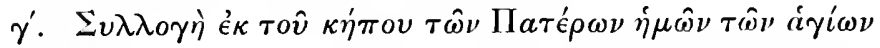

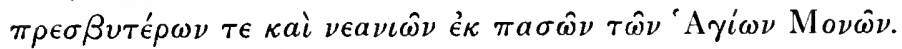

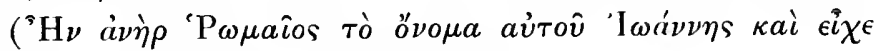

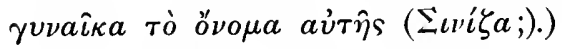

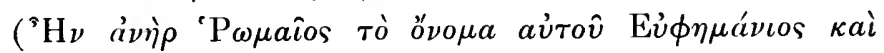
in $\pi 0 \lambda \dot{v} \pi \lambda$ ov́ $\sigma \iota \omega s$.)

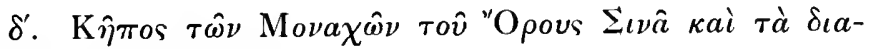

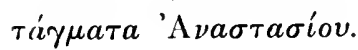




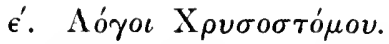

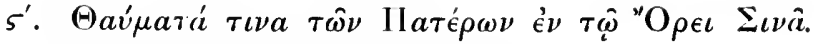

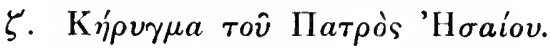

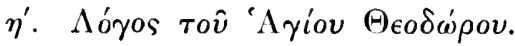

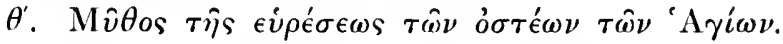

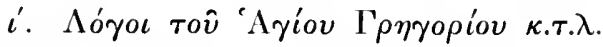

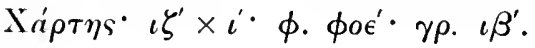

\section{2}

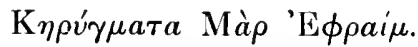

$$
\text { مقالات مار افرام }
$$

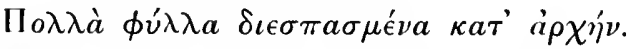

Х $a \rho \tau \eta \varsigma^{\cdot} \iota \eta^{\prime} \times \iota \gamma^{\prime} \phi . \rho \mu \beta^{\prime} \cdot \gamma \rho . \iota \gamma^{\prime}$.

\section{3}

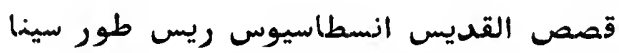

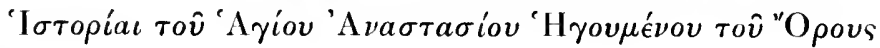
$\Sigma \iota \nu \hat{a}$.

'E $\lambda \lambda \epsilon \iota \pi \grave{\epsilon} s \kappa a \tau^{\prime} \dot{a} \rho \chi \eta^{\prime} \nu$.

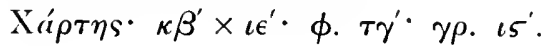

\section{4}

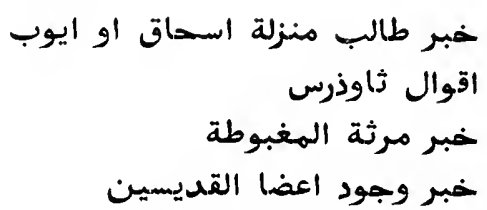

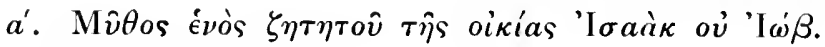

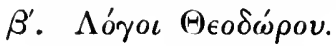

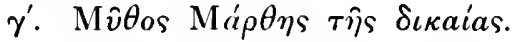

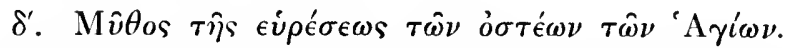

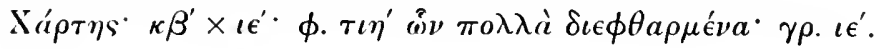




\section{5}

اخبار الابا القديسين النساك الهتوحدين اذطاوسوس ونحوميوس

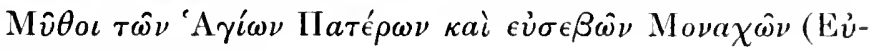

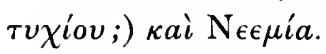

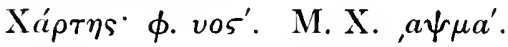

\section{6}

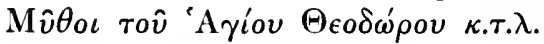

$$
\text { اخبار القديس ثاوذورس والخ }
$$

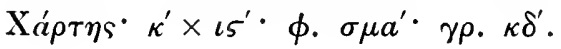

\section{7}

$$
\text { اقوال القديس ذوروثاوس اقليمقس والخ }
$$

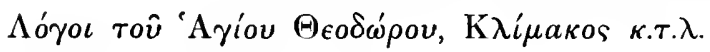

$\mathrm{X} a ́ \rho \tau \eta s^{*} \kappa \beta^{\prime} \times \iota \varsigma^{\prime} \cdot \phi . \rho \iota \zeta^{\prime} \gamma \rho . \kappa^{\prime}$.

\section{8}

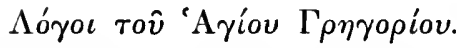

$$
\text { اقوال العديس اغريغوريوس }
$$

$\mathrm{X} a ́ \rho \tau \eta \varsigma^{*} \iota \gamma^{\prime} \times \iota^{\prime} \phi . \sigma \mu \zeta^{\prime} \gamma \rho . \iota a^{\prime}$.

\section{9}

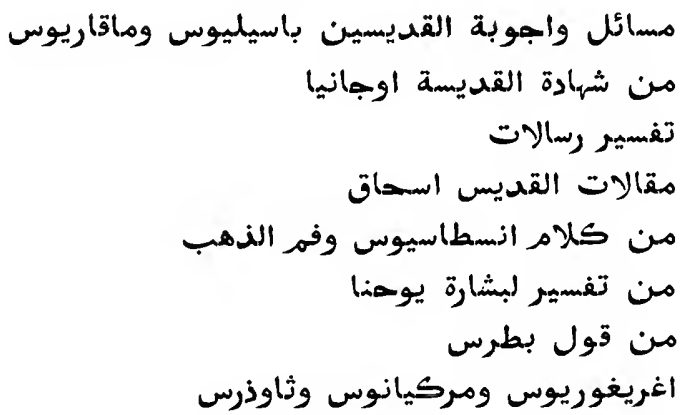

G. A. M. 


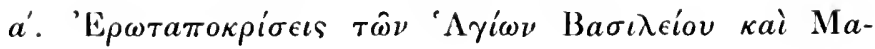
карі́ov.

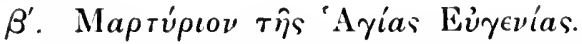

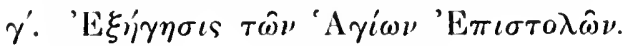

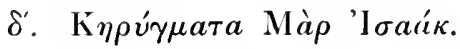

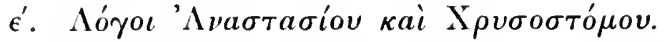

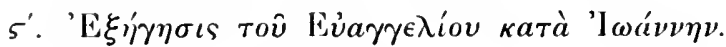

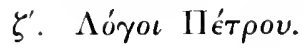

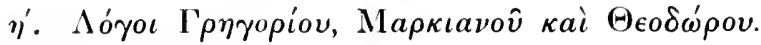

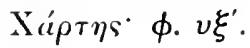

\section{0}

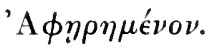

\section{1}

'Iбторі́a той 'A yíov Maкapíov.

$$
\text { قصة القديس مقاريوس }
$$

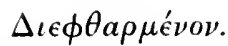

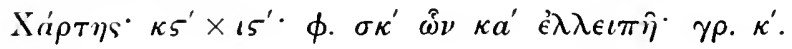

\section{2}

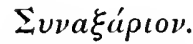

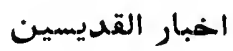

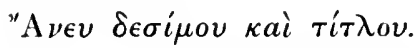

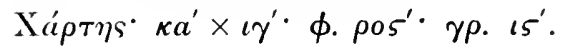

\section{3}

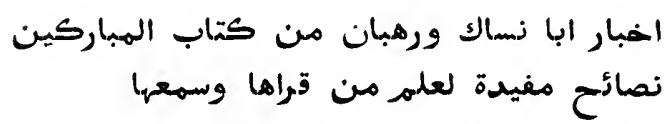

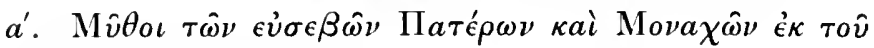

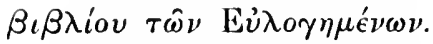

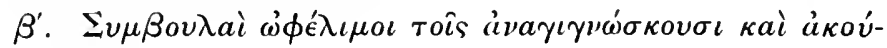

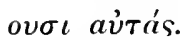

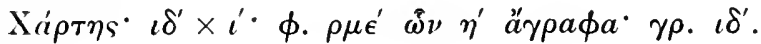




\section{4 \\ 575 \\ 576 \\ 577}

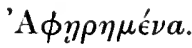

\section{8}

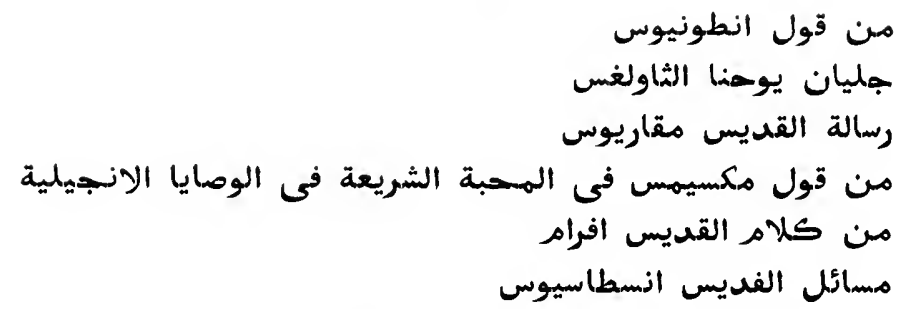

$a^{\prime}$. Lóyos 'A $\nu \tau \omega \nu$ íov.

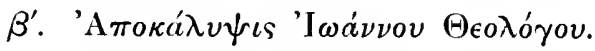

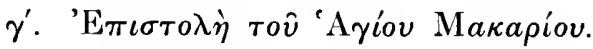

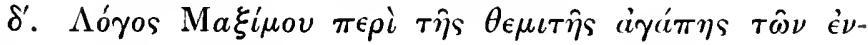

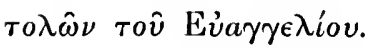

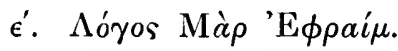

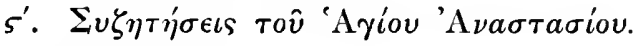

$\Pi o \lambda \dot{v} \epsilon \dot{\epsilon} \lambda \epsilon \iota \pi \epsilon^{\prime}$.

$\mathrm{X} a \dot{\rho} \rho \eta \varsigma^{*} \kappa \varsigma^{\prime} \times \iota \zeta^{\prime} \cdot \phi . \sigma o \zeta^{\prime}$.

\section{$+579$}

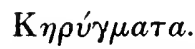

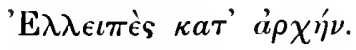

$\mathrm{X} a^{\prime} \rho \tau \eta \varsigma^{\circ} \kappa \gamma^{\prime} \times \iota \varsigma^{\prime} \phi . \rho \pi^{\prime} \cdot \gamma \rho . \iota \eta^{\prime}$.

\section{0}

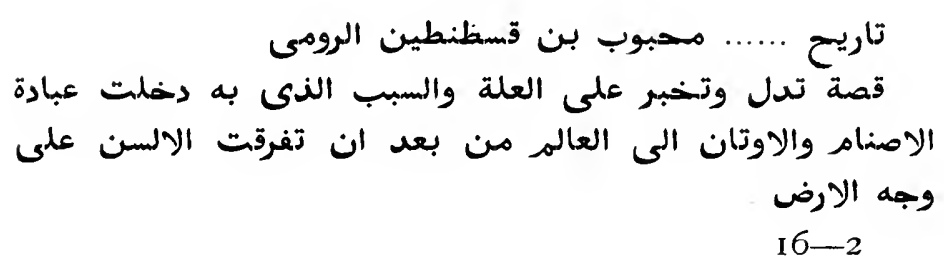




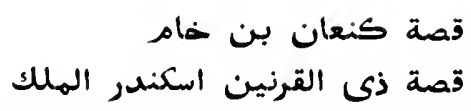

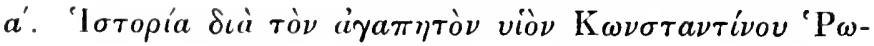
paíov.

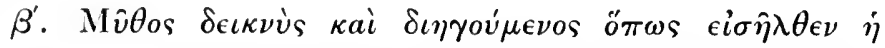

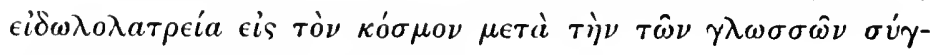

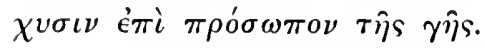

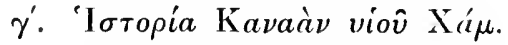

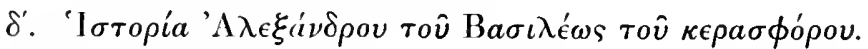

$\mathrm{X} \dot{\rho} \rho \tau \eta \varsigma^{\circ} \kappa^{\prime} \times 1 \varsigma^{\prime} \phi . \sigma \varsigma^{\prime} \cdot \gamma \rho . \theta^{\prime}$.

\section{1}

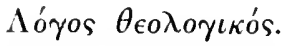

"А

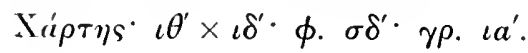

\section{2}

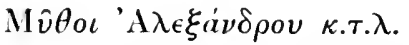

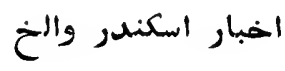

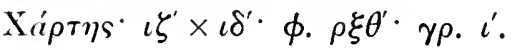

\section{3 \\ $584\}$}

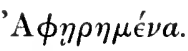

\section{5}

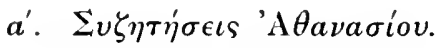

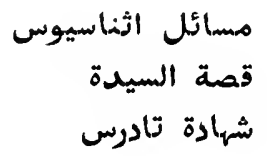

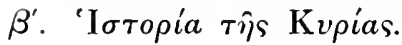

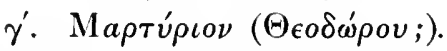

$\mathrm{X} a \dot{\rho} \tau \eta \varsigma^{\circ} \iota \zeta^{\prime} \times \iota \gamma^{\prime} \quad \phi . \quad \rho \nu \beta^{\prime} \cdot \gamma \rho . \quad \delta^{\prime} \kappa a i \quad \iota \epsilon^{\prime} \kappa a i \iota a^{\prime}$. M. X. $a \rho \xi \gamma^{\prime}$. 
586

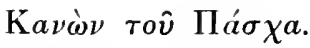

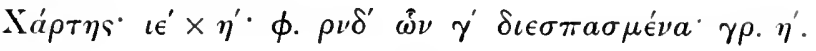

\section{7}

$$
\text { صروف }
$$

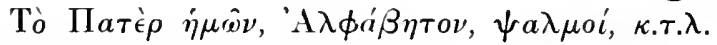

$\mathrm{X} a ́ \rho \tau \eta \varsigma^{\cdot} \iota \delta^{\prime} \times \iota \eta^{\prime} \cdot \phi . \kappa \gamma^{\prime} \cdot \gamma \rho . \iota \beta^{\prime} . \quad$ M. X. $a \psi \pi \zeta^{\prime}$.

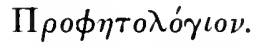

\section{$+588$}

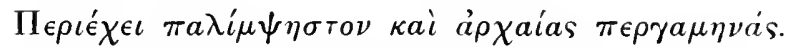

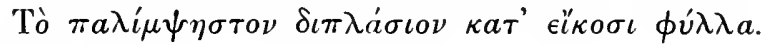

М $\epsilon \mu \beta \rho a \dot{a} \eta^{\cdot} \kappa \gamma^{\prime} \times \iota \zeta^{\prime} \cdot \phi \cdot \rho \iota \zeta^{\prime} \cdot \gamma \rho . \kappa a^{\prime}$.

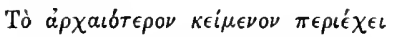

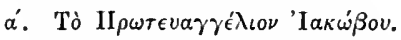

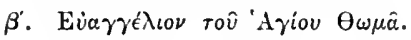

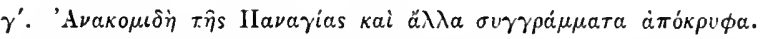

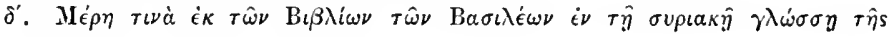

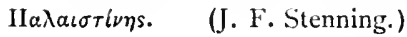

\section{9}

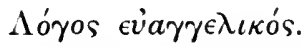

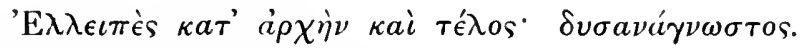

$\mathrm{X} a \dot{\rho} \rho \eta \varsigma^{*} \kappa \zeta^{\prime} \times \iota \zeta^{\prime} \cdot \phi . o^{\prime} \cdot \gamma \rho . \kappa^{\prime}$.

\section{0}

Kavóves.

Ха́ $\rho \tau \eta \varsigma^{*} \kappa a^{\prime} \times \iota \delta^{\prime} \cdot \phi . \rho \xi \delta^{\prime} \cdot \gamma \rho . \iota \theta^{\prime}$.

\section{1}

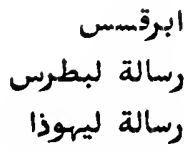




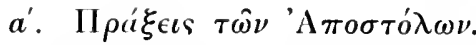

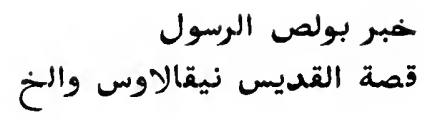

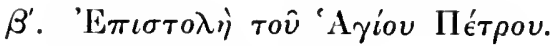

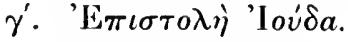

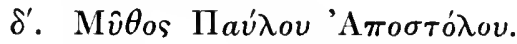

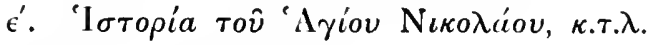

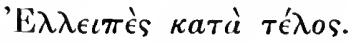

Х'́́ $\rho \tau \eta \varsigma^{\cdot} \kappa \beta^{\prime} \times \iota \varsigma^{\prime} \cdot \phi . \rho \lambda \zeta^{\prime} \cdot \gamma \rho . \iota \epsilon^{\prime}$.

\section{2}

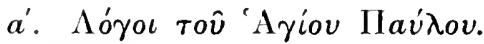

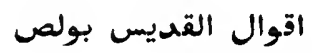

مقالات

$\beta^{\prime} . \mathrm{K} \eta \rho \dot{\gamma} \mu a \tau a$.

"А

Хá $\rho \tau \eta \varsigma^{\circ} \phi . \rho \mu \varsigma^{\prime}$.

\section{3}

$\mathrm{K} \eta \rho \dot{\gamma} \mu a \tau a$.

"А

Х $a^{\prime} \rho \tau \eta s^{\cdot} \kappa \beta^{\prime} \times \iota \epsilon^{\prime} \cdot \phi . \rho o \zeta^{\prime} \cdot \gamma \rho . \iota \eta^{\prime}$.

\section{4}

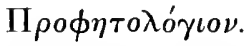

Хá $\rho \tau \eta \varsigma^{\cdot} \kappa \varsigma^{\prime} \times \iota \eta^{\prime} \cdot \phi . \rho \mu \zeta^{\prime} \cdot \gamma \rho . \iota \eta^{\prime}$.

\section{5}

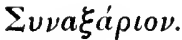

$\mathrm{X} a \dot{\rho} \rho \tau \eta \varsigma^{\circ} \kappa \gamma^{\prime} \times \iota \varsigma^{\prime} \phi . \sigma \iota \epsilon^{\prime} \gamma \rho . \iota \delta^{\prime}$.

\section{6}

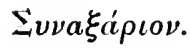

$\mathrm{X} a ́ \rho \tau \eta s^{*} \kappa \eta^{\prime} \times \kappa \beta^{\prime} \cdot \phi \cdot \rho \iota \beta^{\prime} \cdot \gamma \rho \cdot \iota 5^{\prime}$. 


\section{7}

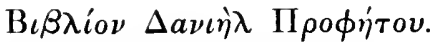

$$
\text { ختاب دانيال النبى العالم }
$$

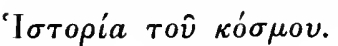

$\mathrm{X} \alpha \dot{\rho} \rho \tau \eta \varsigma^{\cdot} \kappa \delta^{\prime} \times 15^{\prime} \cdot \phi \cdot \rho \nu \epsilon^{\prime} \cdot \gamma \rho .15^{\prime}$.

\section{8}

Kavóves.

$\mathrm{X} \alpha \dot{\alpha} \rho \tau \eta \varsigma^{\cdot} \kappa \delta^{\prime} \times \iota \zeta^{\prime} \cdot \phi . \rho \eta^{\prime} \cdot \gamma \rho . \iota \epsilon^{\prime}$.

$$
+599
$$

رسالة يوحنا القديس الدمشقى الى قزما اسقف مدينة مايوية

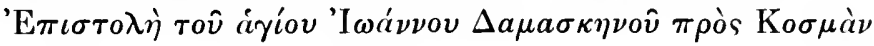

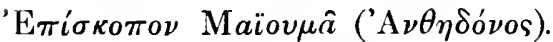

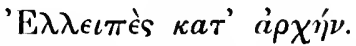

$\mathrm{X} \alpha \dot{\alpha} \rho \eta \varsigma^{\cdot} \iota \zeta^{\prime} \times \iota \gamma^{\prime} \cdot \phi . \tau \xi \eta^{\prime} \cdot \gamma \rho . \iota \delta^{\prime}$.

\section{0}

Kavóves.

$\mathrm{X} a ́ \rho \tau \eta \varsigma^{*} \iota \varsigma^{\prime} \times \iota \beta^{\prime} \cdot \phi . \tau \iota \varsigma^{\prime} \gamma \rho . \iota a^{\prime}$.

\section{1}

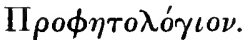

$\mathrm{X} a \rho \tau \eta \varsigma^{\cdot} \kappa^{\prime} \times \imath \delta^{\prime} \cdot \phi . \mu \theta^{\prime} \cdot \gamma \rho . \iota \beta^{\prime}$.

602

خبر مقلد الينا اناس مادقين مشهود لهم بالورع يذكر فيه وجود نقل راس القديس يوحنا سابق الرب الري

شهادة القديسة افطوكية القديس يورية

ذكر تصرف مريم الهصرية في عيشتها

خبر تصرف طايسية الزانية

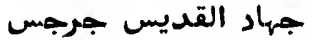




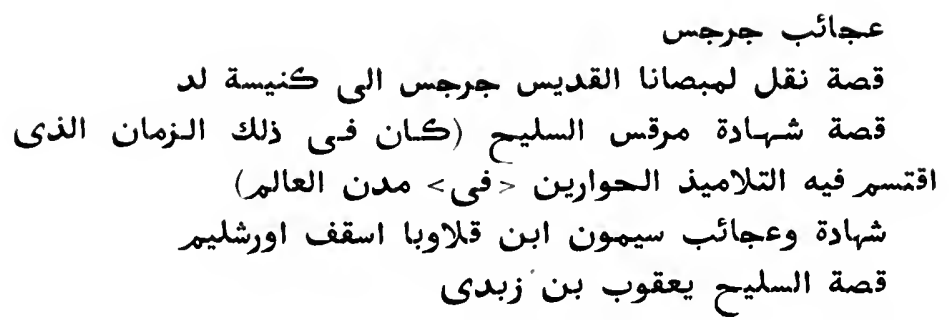

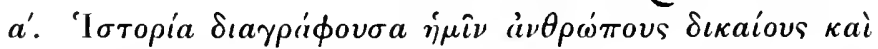

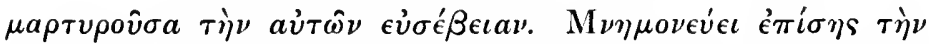

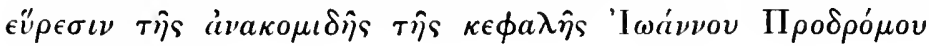
Tov̂ Kupíov.

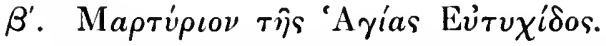

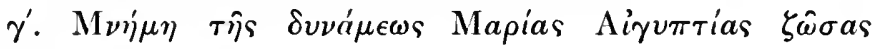
av่าîs.

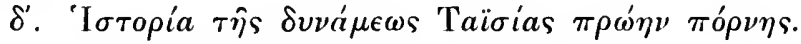

'. 'A

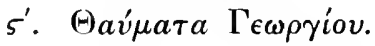

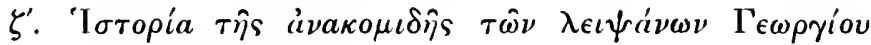

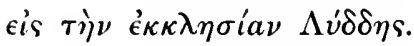

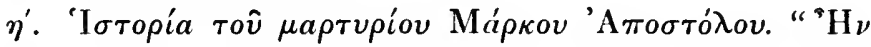

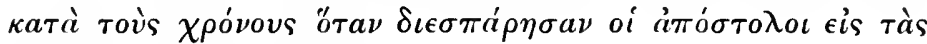

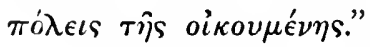

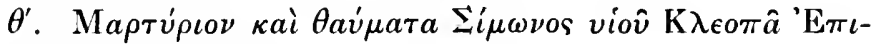
бко́тог 'Т

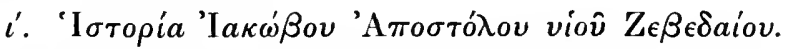

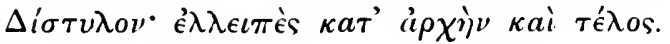

$\mathrm{X} \alpha^{\prime} \rho \tau \eta \varsigma^{\cdot} \lambda a^{\prime} \times \kappa \beta^{\prime} \cdot \phi . \rho \kappa^{\prime} \gamma \rho . \kappa \beta^{\prime}$.

$\left.\begin{array}{l}603 \\ 604 \\ 605 \\ 606 \\ 607 \\ 608 \\ 610\end{array}\right\}$

"E $\nu \tau v \pi \pi$. 


\section{1}

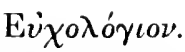

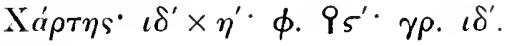

\section{9}

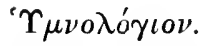

"A $\nu \epsilon v \delta \epsilon \sigma i \mu o v$.

$\mathrm{X} a \dot{\rho} \tau \eta s^{\cdot} \iota \epsilon^{\prime} \times \iota^{\prime} \phi \phi . \rho \nu \delta^{\prime} \cdot \gamma \rho . \quad \imath \gamma^{\prime}$.

\section{0}

$\mathrm{K} a \nu \omega \dot{\nu} \tau o \hat{v} \Pi a \dot{\sigma} \chi^{a}$.

قنون الفصح

$\mathrm{X} \alpha \rho \rho \eta s^{\circ} \iota \epsilon^{\prime} \times \iota^{\prime} \phi . \rho \mu \theta^{\prime} \cdot \gamma \rho .15^{\prime}$.

\section{1}

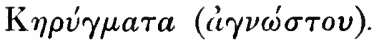

$\mathrm{X} \alpha \rho \rho \eta s^{\cdot} \iota \zeta^{\prime} \times \iota \beta^{\prime} \phi . \rho \mu \gamma^{\prime} \gamma \rho . \iota \beta$.

\section{2}

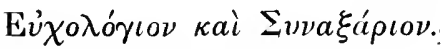

$\mathrm{X} \alpha \rho \rho \eta s^{\cdot} \iota \beta^{\prime} \times \theta^{\prime} \cdot \phi . \sigma \lambda^{\prime} \cdot \gamma \rho . \iota a^{\prime}$.

\section{3}

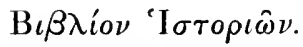

$\mathrm{X} \alpha \rho \tau \eta \varsigma^{\cdot} \kappa a^{\prime} \times \iota \gamma^{\prime} \phi . \pi a^{\prime} \gamma \rho . \iota \zeta^{\prime}$.

\section{4}

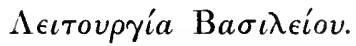

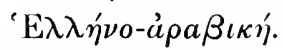

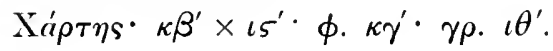

\section{5}

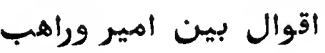

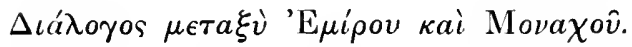

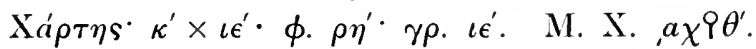

G. A. M. 


\section{6}

ترجهة رسالة العبرانيين الذى ارسيلما الرسول بولص الى اليهود

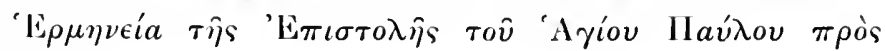
'EBpaious.

$\mathrm{X}^{\prime} \rho \tau \eta \varsigma^{\circ} \lambda a^{\prime} \times \kappa \beta^{\prime} \cdot \phi . \sigma \lambda^{\prime} \cdot \gamma \rho . \kappa a^{\prime}$. M. X. $a \psi \kappa s^{\prime}$.

\section{7}

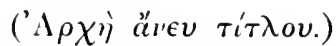

الهى صفى الهسيح بلاسيوس محب الله وزير الهلك الهختمّار

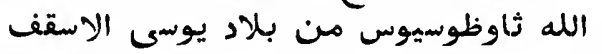

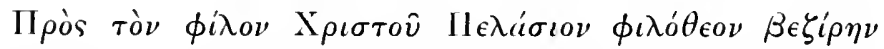

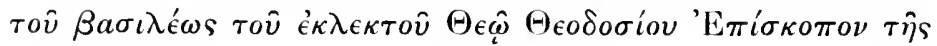
$\gamma \hat{\eta} s$ ('A $\nu \sigma i o v ;)$.

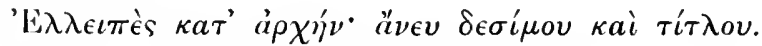

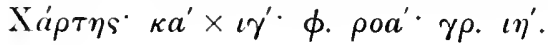

\section{8}

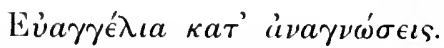

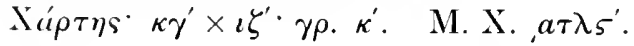

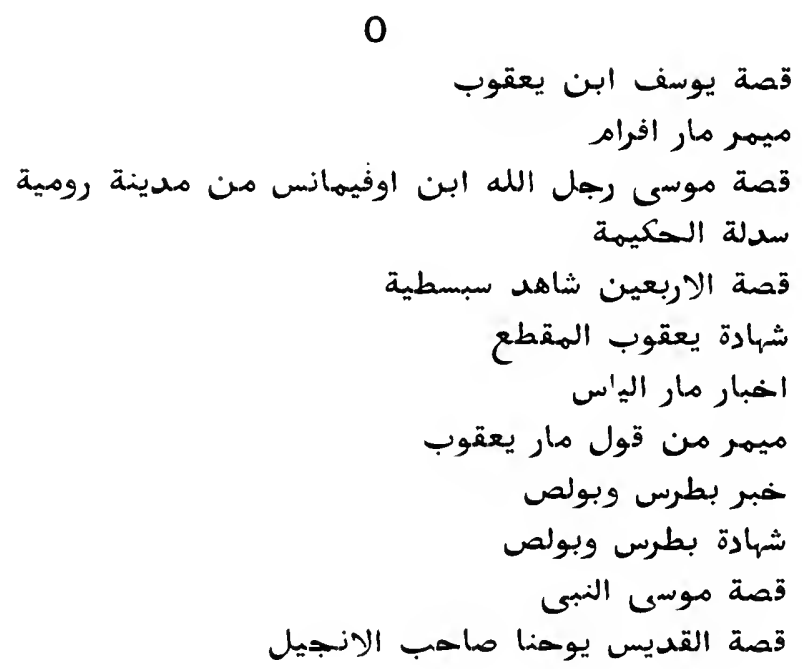




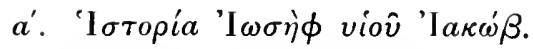

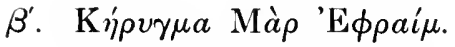

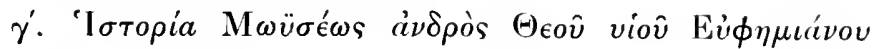

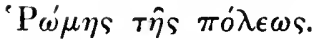

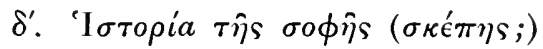

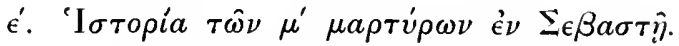

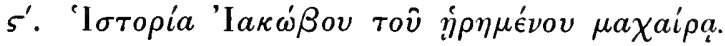

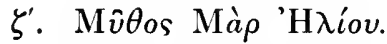

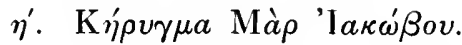

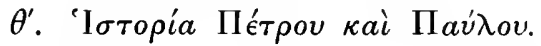

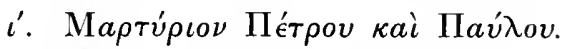

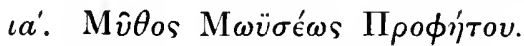

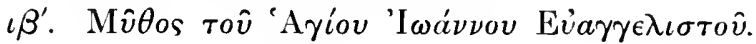

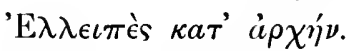

$\mathrm{X} \dot{\alpha} \rho \tau \eta \varsigma^{\bullet} \phi . \sigma \kappa \delta^{\prime}$. 



\section{IINA $\Xi$.}

\section{KAT' A $\Lambda$ TABHTON.}

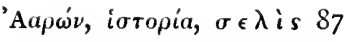

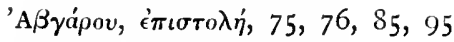

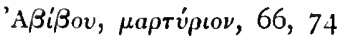

'A $\beta$ paá $\mu$, I, 88

'A $\gamma \gamma \hat{\epsilon} \lambda \omega \nu, \omega \delta a i$, IO I

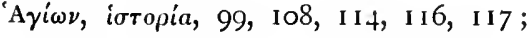

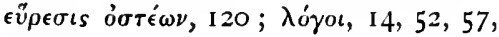
9I, I I 7

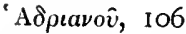

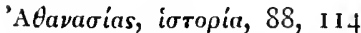

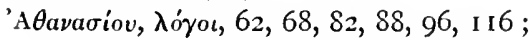

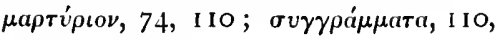
I $13 ; \sigma v \zeta \eta \tau \dot{\eta} \sigma \epsilon \iota s, 124$

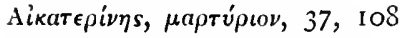

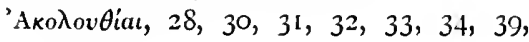
87, 1 i 8

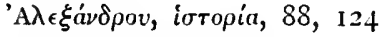

'A $\epsilon \epsilon \xi i o v$, iбторía, $7 \mathrm{I}$

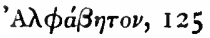

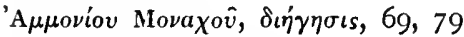

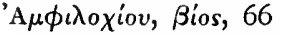

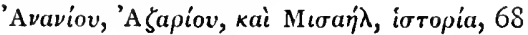

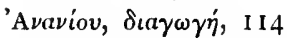

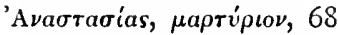

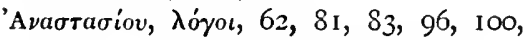

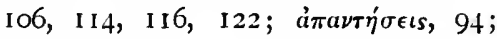

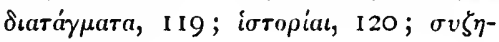

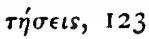

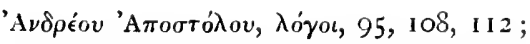

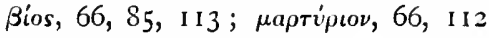

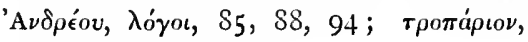

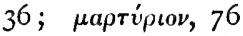

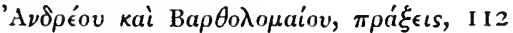

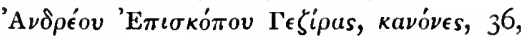
37,94

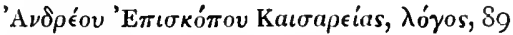

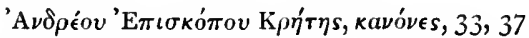

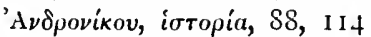

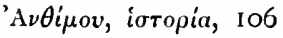

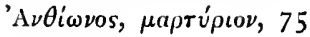

"A $\nu \nu a, 37$

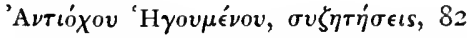

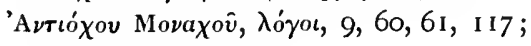

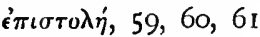

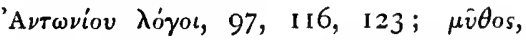

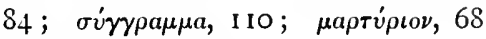
'А

'А $\rho \dot{\epsilon} \theta a ; \lambda$; $\gamma o \iota$, I I 5

'А

'A

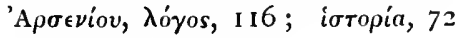

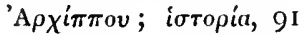

'А $\sigma \kappa \eta \tau \hat{\omega} \nu ; \beta \iota \beta \lambda i ́ \nu$, Iоо

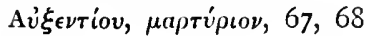

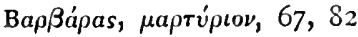




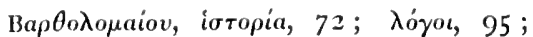
$\kappa \eta ́ \rho v \xi \iota, 113$

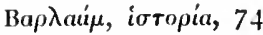

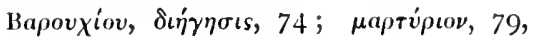
I 13

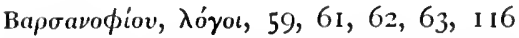

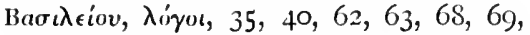
$79,82,83,87,89,93,94,97$, 100,

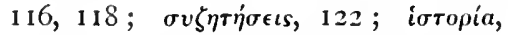

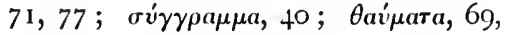
82, $109 ; \lambda \in$ tтоирүiı, 129

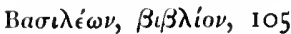

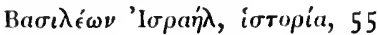

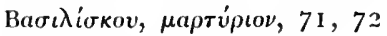

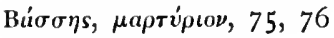

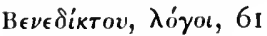

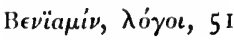

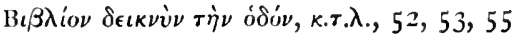

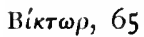

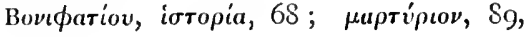
93

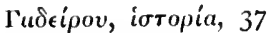

Г'́⿻

Ге $\rho \mu a \nu o \hat{v}, \lambda o ́ \gamma o s, 75$

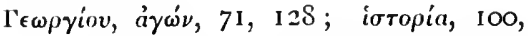

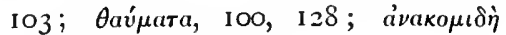

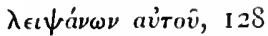

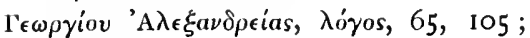
iбтopía, 105

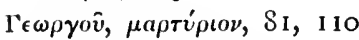

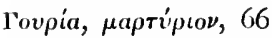

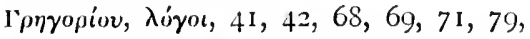
82, 84, 93, 94, 116, 120, 121, I 22 ; є̇ $\tau \eta \dot{\sigma e \iota s, ~} 95$

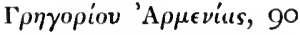

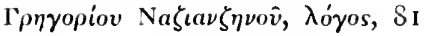

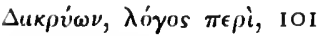

$\Delta a v i \dot{\eta} \lambda$ II

$\Delta a \nu i \dot{\lambda} \Sigma \tau v \lambda i \tau \eta s, 67$

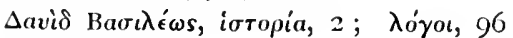

$\Delta \epsilon \hat{\imath} \gamma \mu a, \kappa . \tau . \lambda .$, I I

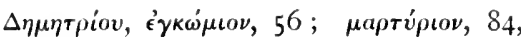
I 10

$\Delta \iota a \theta \dot{\eta} \kappa \eta, \dot{\eta} \pi a \lambda a \iota \dot{a}, 3$

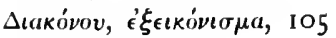

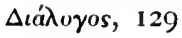

$\Delta \iota \delta a \sigma \kappa a \lambda i a, 38,50$

$\Delta \iota \delta a \chi \dot{\eta} \tau \bar{\eta} s$ Х

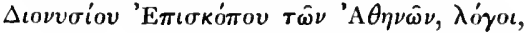

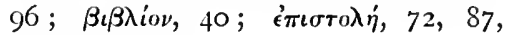

93, 94, 99, 1 I 2

$\Delta \iota \omega \dot{\xi} \epsilon \omega s$, iбropía, 102

$\Delta o \mu \in \tau i o v$, iotopía, 35

$\Delta \omega \rho о \theta \dot{a} a s, \mu a \rho \tau \dot{\rho} \iota о \nu, 90$

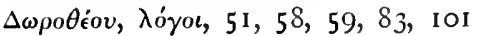

'Eßópous; ioropía, 107

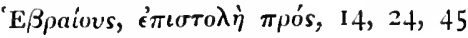

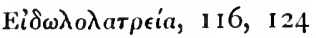

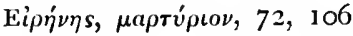

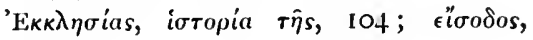
I IO

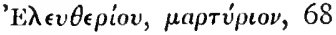

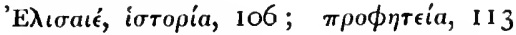

'E $\lambda \lambda \eta \nu i \sigma \tau i, 20,26$

'E $\xi \eta \gamma \eta \dot{\sigma} \sigma \iota$, IOO, 122

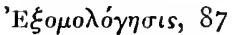

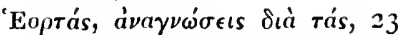

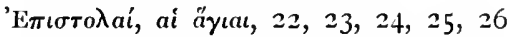

'E $\pi \iota \sigma \tau o \lambda \dot{\eta}$ ảiò $\tau \hat{\omega} \nu$ ov่ $\rho a \nu \hat{\omega} \nu, 96$

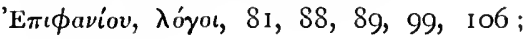
iorropía, 99

'E $p \mu \eta \nu \epsilon i a l, 2,16,20,24,36,41,130$

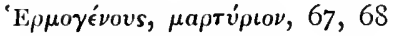

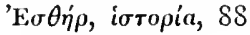

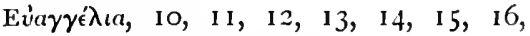
I 7, 18, 19, 20, 21, 22, 130

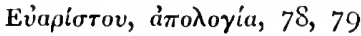

Ev̉ßírov, iбторі́a, 68

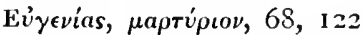

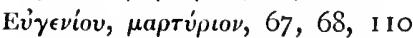

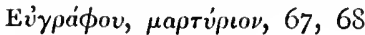

Eviookias, ßíos, 106

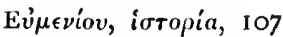

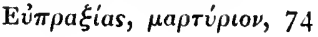




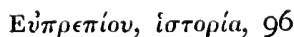

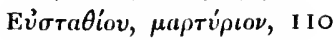

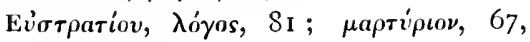
68, 110,119

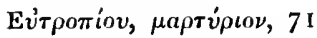

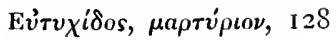

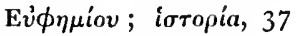

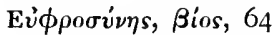

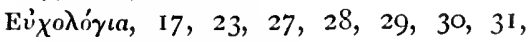
33, 34, 38, 102, I 29

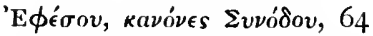

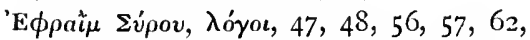
$63,66,74,8_{1}, 82,83,84,85,88,9 \mathrm{I}$, 93, 99, I09, I I4, I I6, I I 8 , I 20, I23,

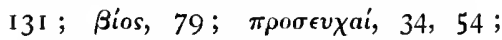

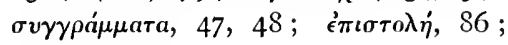
$\sigma v \zeta \eta \tau \dot{\eta} \sigma \epsilon \iota s, 98$

\section{$\mathrm{Z} \eta \dot{\tau} \eta \mu a, 84$}

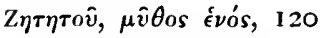

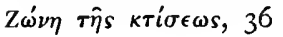

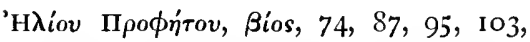

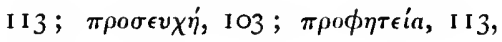
I 4

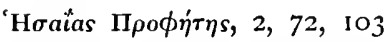

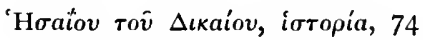

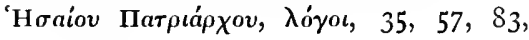
IOI, 120

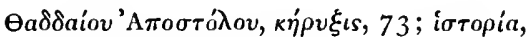

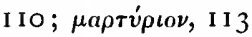

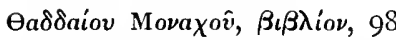

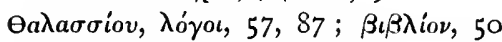

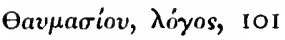

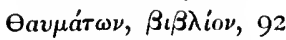

Өє́к $\lambda \eta s$, Bíos, 64, 89

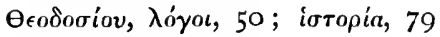

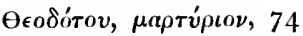

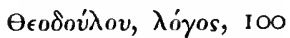

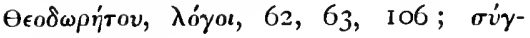
$\gamma \rho а \mu \mu a, 70$

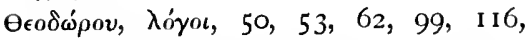

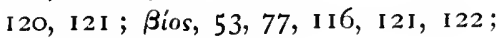

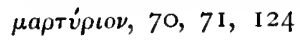

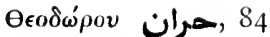

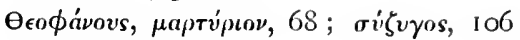

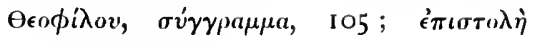
$\pi \rho \grave{s}$ av่тó

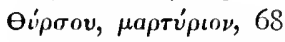

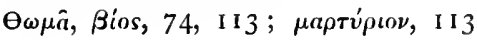

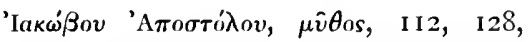

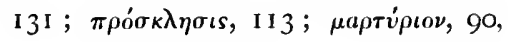

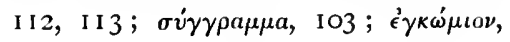
72

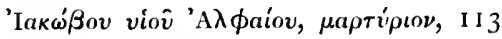

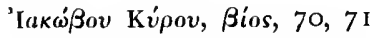

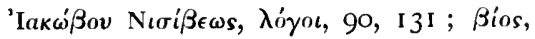
69

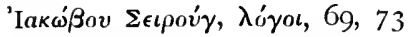

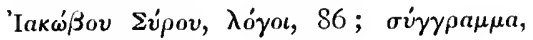
90

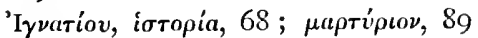

'I $\epsilon \zeta \epsilon \iota \grave{\eta} \lambda \Pi \rho \circ \phi \dot{\eta} \tau \eta s$, I

'Í́ $\rho$ akos ; I I 5

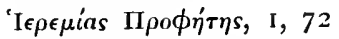

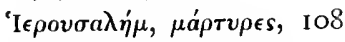

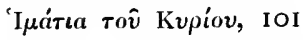

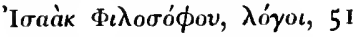

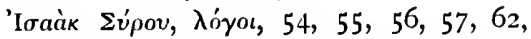
$8 \mathrm{I}, 85,88,96$, IOI, 122 ; $\sigma v \zeta \eta \tau \dot{\eta} \sigma \epsilon \iota s$, 56

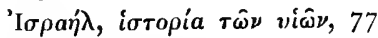

'I

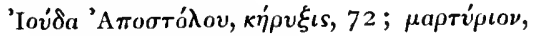
$113 ; \epsilon \dot{\epsilon} \iota \sigma \tau o \lambda \eta \dot{\eta}, 126$

'Iovגıavn', 68

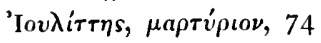

'Iov́ $\sigma \tau \eta s, \mu$ нати́

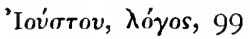

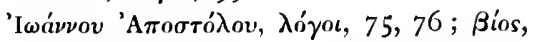

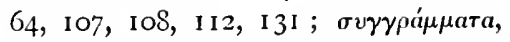

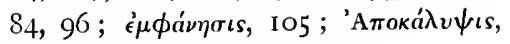

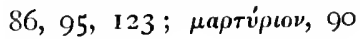

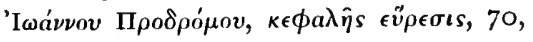
$7 \mathbf{1}$, So, 128

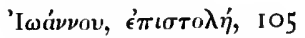

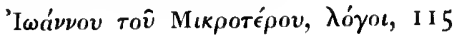


'I 49, 75, 76, 8S, 94, 99, Іо9, І го; $\pi \rho \circ \sigma$.

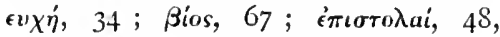

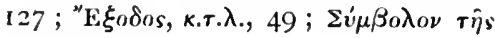

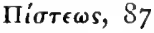

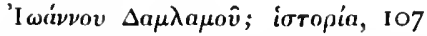

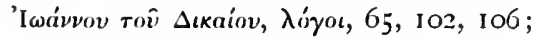

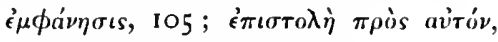

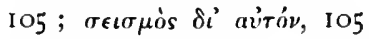

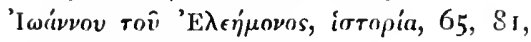

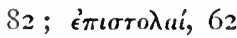

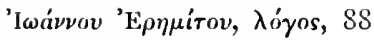

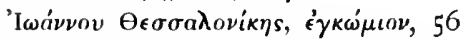

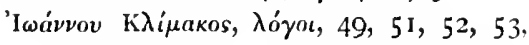
$54,56,62,71,82,83,96$, I 16, I 21 ; Bios, 52, 53, 54; kavóves, 62, 63, 64 ;

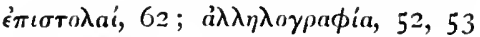

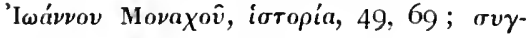

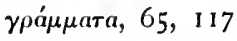

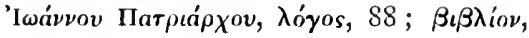
63

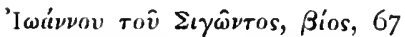

'I $42,43,44,45,46,62,63,65,68,69$, $7 \mathrm{I}, 72,73,74,75,76,79$, So, II $_{22}$, $8_{3}, 84,85,86,87,88,89,91,92,93$, 96, 99, 100, 102, 103, 105, 106, I 10,

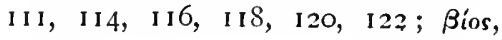

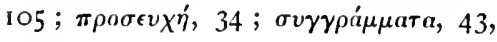
90

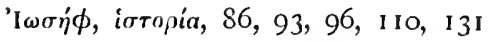

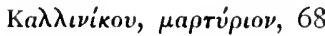

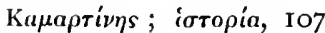

Kavaáv, i $\sigma r o p i ́ a, ~ I 24$

Kavóvยs, 19, 28, 33, 34, 35, 36, 37, 38, $62,63,78,88,96,125,127$

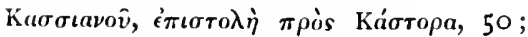

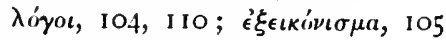

Kє $\lambda \epsilon \sigma \tau i \nu o v, \epsilon ่ \pi เ \sigma \tau o \lambda \dot{\eta}, 92$

Kंрú $\mu a \tau a, 21,22,42,43,44,45,46$, 5 I, 58, 59, 8I , 83, 91, 97, I I 4, 123 , 126, 129

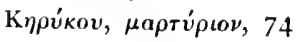

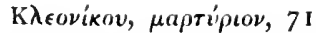

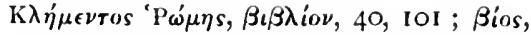

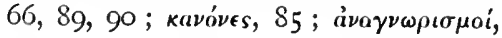
IOI

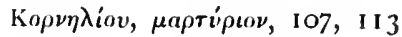

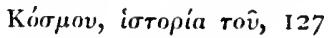

Kovtías, ioropía, 71

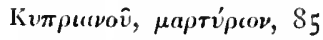

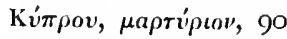

Kvрєакой, Bios, 64

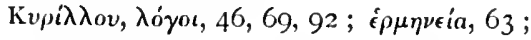

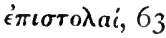

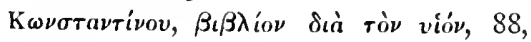
I 24

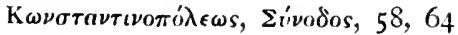

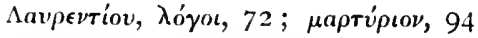

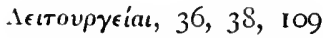

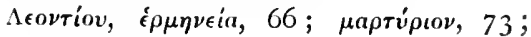
$\phi \lambda$ vapía, 74

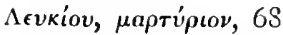

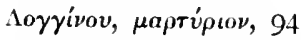

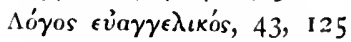

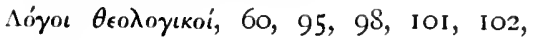
I 24

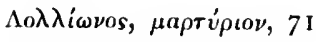

Аоขкâ 'А $\mu \iota \% \nu, 94$

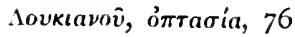

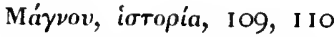

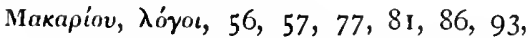

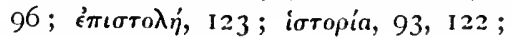
$\sigma v \zeta \eta \tau \dot{\sigma} \sigma \epsilon t s, 122$

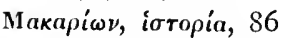

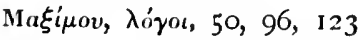

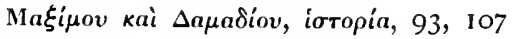

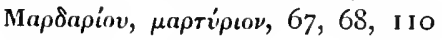

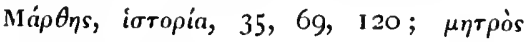
$\Sigma v \mu \kappa \omega \dot{\nu}, 74$

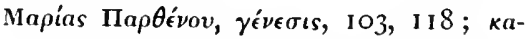

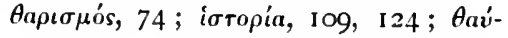

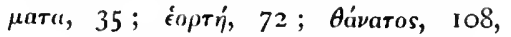

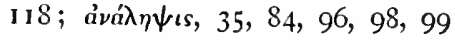


Mapias Aigutrias, $\mu \nu \eta^{\prime} \eta \eta, 7$ I, 80, 102, I 28

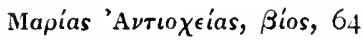

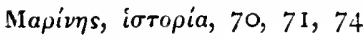

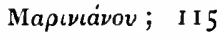

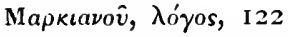

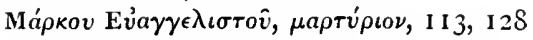

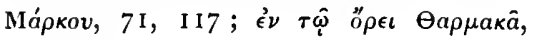

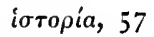

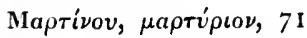

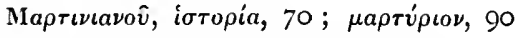

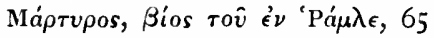

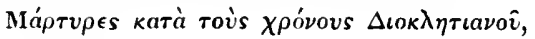
74

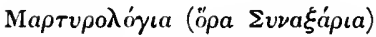

Martaiov 'A

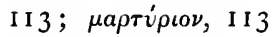

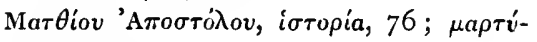

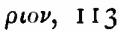

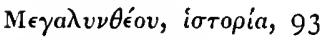

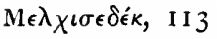

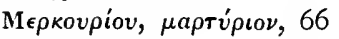

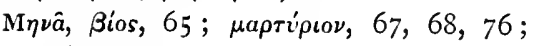

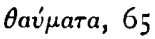

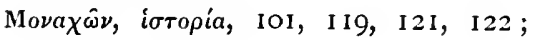

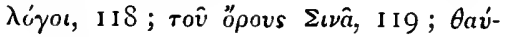

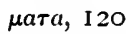

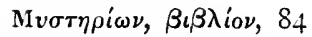

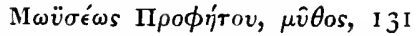

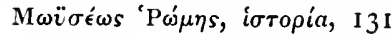

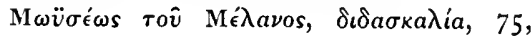
76

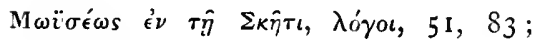

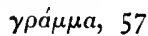

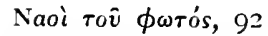

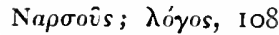

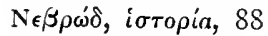

Neì

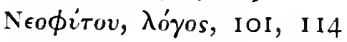

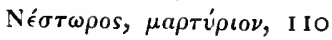

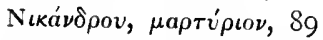

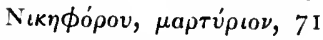

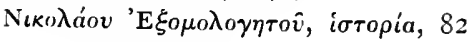

G. A. M.

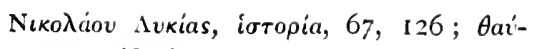
ната, 68, 82

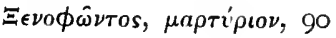

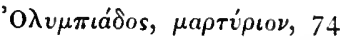

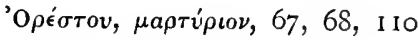

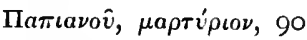

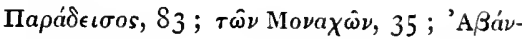

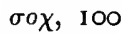

Парочнíaı, 3

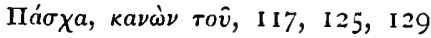

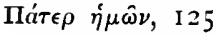

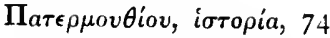

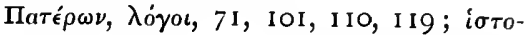

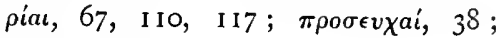

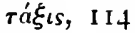

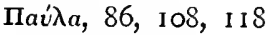

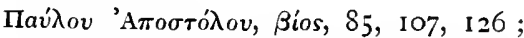

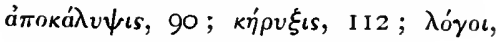
126 ; є̇ $\pi \iota \sigma \tau o \lambda a i, ~ 7, ~ 14,22,23,24,25$,

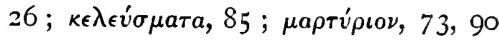

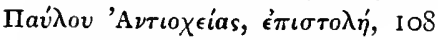

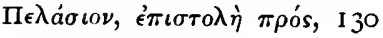

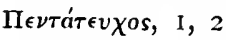

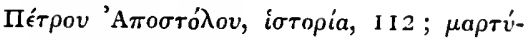

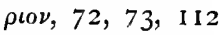

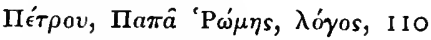

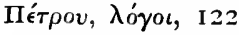

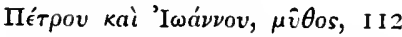

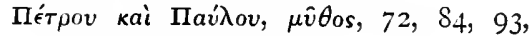

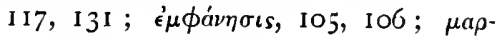

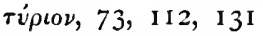

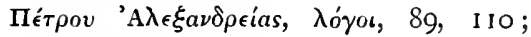

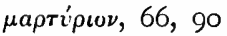

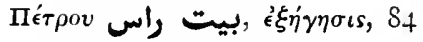

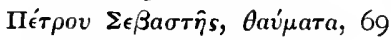

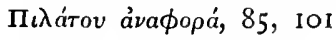

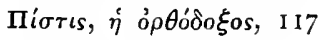

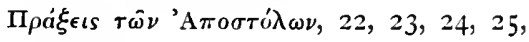
26, 126

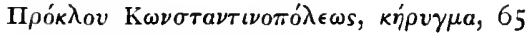

Прокотіоv, iбторі́a, I Iо 
$13 \mathrm{~S}$

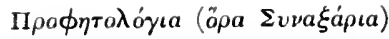

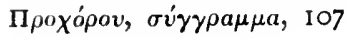

$\Pi \rho \omega \tau \epsilon \operatorname{va\gamma \gamma \hat {\epsilon }\lambda เo\nu ,84}$

'P $\omega \mu a \nu o v$, Bíos, 66

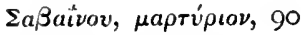

$\Sigma a ́ \beta \beta a, \mu a \rho \tau i ́ p t o v, 89$

Saßivav, Bios, 64

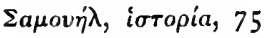

$\Sigma a \mu \omega \nu \bar{a}, \mu a \rho \tau \dot{\rho} \rho t o \nu, 66$

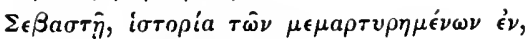
8o, 103, IO4, I IO, I3 I

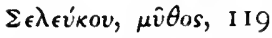

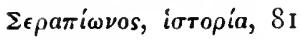

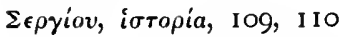

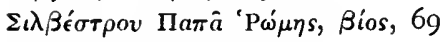

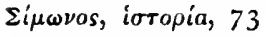

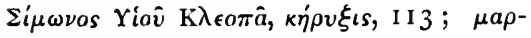

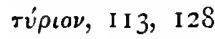

Si $\mu \omega \nu o s$ 'E $\rho \eta \mu i \tau o v, \sigma \nu \zeta \eta \tau \dot{\eta} \sigma \epsilon \iota s, 5 \mathrm{I}$

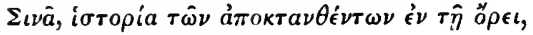
70,79, II 4, II 8

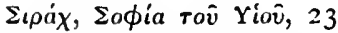

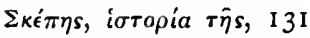

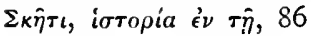

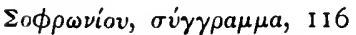

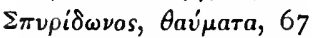

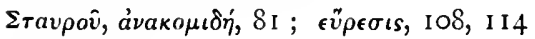

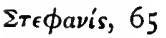

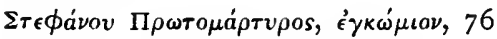

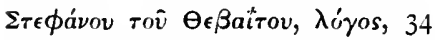

$\Sigma v \zeta \eta \tau \eta \dot{\sigma} \sigma \epsilon$ เ, I $, 56,57,6 \mathrm{I}, 8 \mathrm{I}, 9 \mathrm{I}, 94$, 97, 98, I I 8

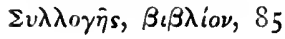

TINAE.

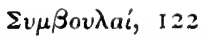

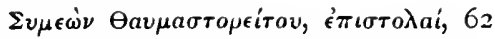

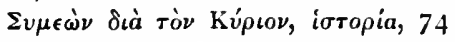

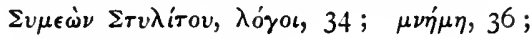
$\sigma v \zeta \eta \tau \dot{\eta} \sigma \epsilon \iota s, 5 \mathrm{I}, 56$

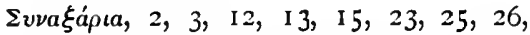
$27,28,29,3 \mathrm{I}, 32,34,35,36,38,44$, 77, 78, 79, 80, 87, 90, 91, 93, 95, 98, I 22, I 25, I 26, 127

Evplorí, 19

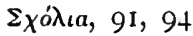

Taï $\sigma i a s, \mu \nu \eta \dot{\mu} \eta, 7 \mathrm{I}, 128$

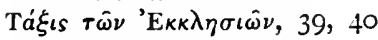

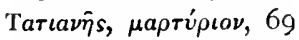

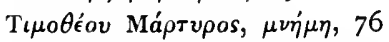

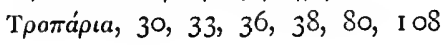

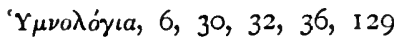

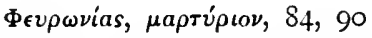

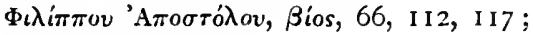

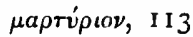

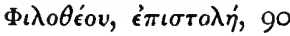

Xápıtos, ßíos, 64

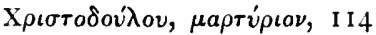

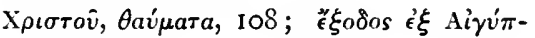
тov, IOS

$\Psi a \lambda \tau \dot{\eta} \rho \iota a, 3,4,5,6,7,8,9,10,24,27$, $28,29,30,31,32,33,125$

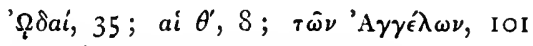

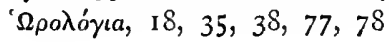





\section{Studia Sinaitica.}

I. A CATAlogue OF THE SYRIAC MSS. IN THE CONVENT of St Catharine on Mount Sinai. Compiled by Agnes Smith Lewis. I'rice 10s. 6 . net.

I1. AN ARABIC VERSION OF ST PAUL'S EPISTLES TO THE Romans, Corinthians, Galatians, and part of Ephesians. Edited from a Ninth century MS. in the Convent of St Catharine on Mount Sinai by Margaret Dunlop Gibson. Price 5s. net.

III. A CATALOGUE OF THE ARABIC MSS. IN THE CONVENT of St Catharine on Mount Sinai. Compiled by MARGareT Dunlop Gibson. Price 6s. net.

IV. A TRACT OF PLUTARCH ON THE ADVANTAGE TO BE derived from one's Enemies. By Dr Eberhard Nestle. Price 2s. net.

Other numbers of the Series are in course of preparation. 



\section{RETURN CIRCULATION DEPARTMENT}

TO $\rightarrow 202$ Main Library

\begin{tabular}{l|l|l}
\hline $\begin{array}{c}\text { LOAN PERIOD 1 } \\
\text { HOME USE }\end{array}$ & 2 & 3 \\
\hline 4 & 5 & 6 \\
\hline
\end{tabular}

ALL BOOKS MAY BE RECALLED AFTER 7 DAYS

morith loons muy be renewed by colling 042.3405

- month louns may be recharaed by bringing books to Circulation Desk

Renewais and recharges may be mode 4 doys prior 10 due date

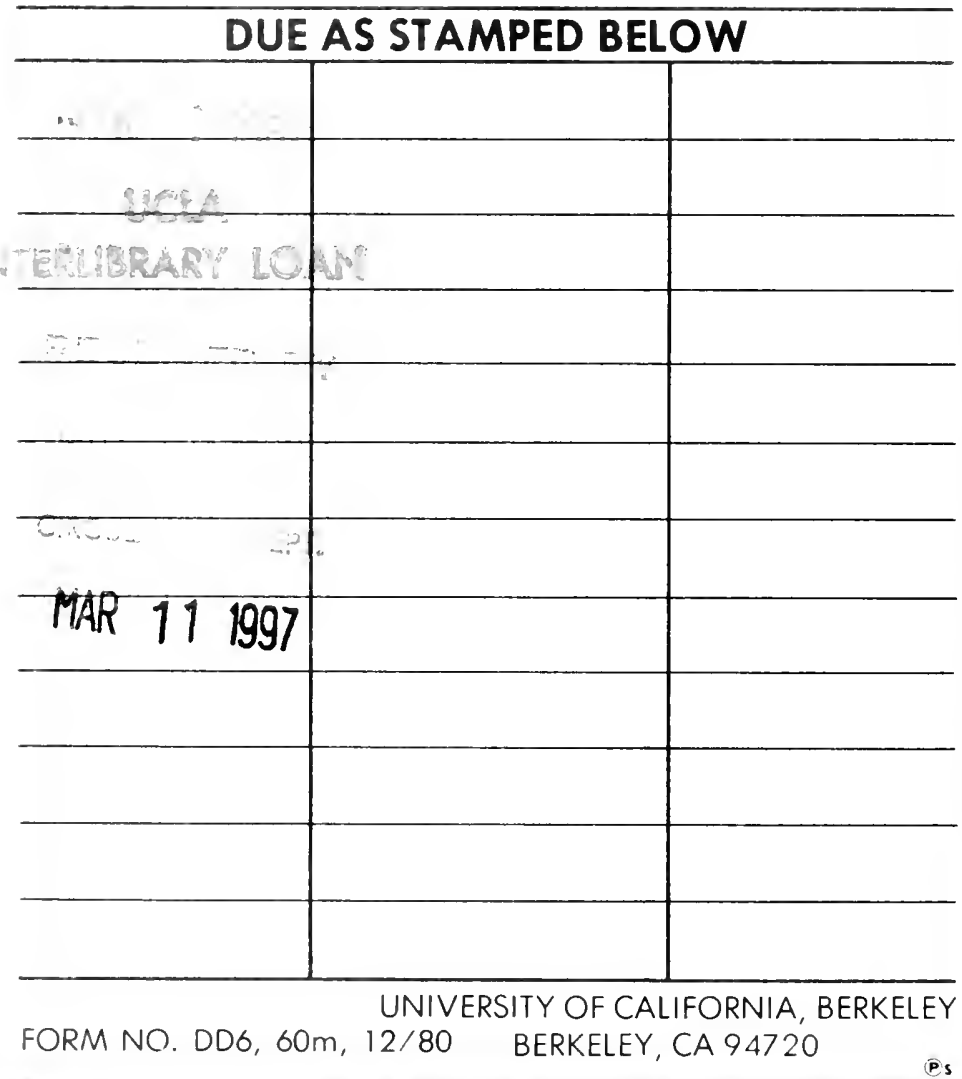




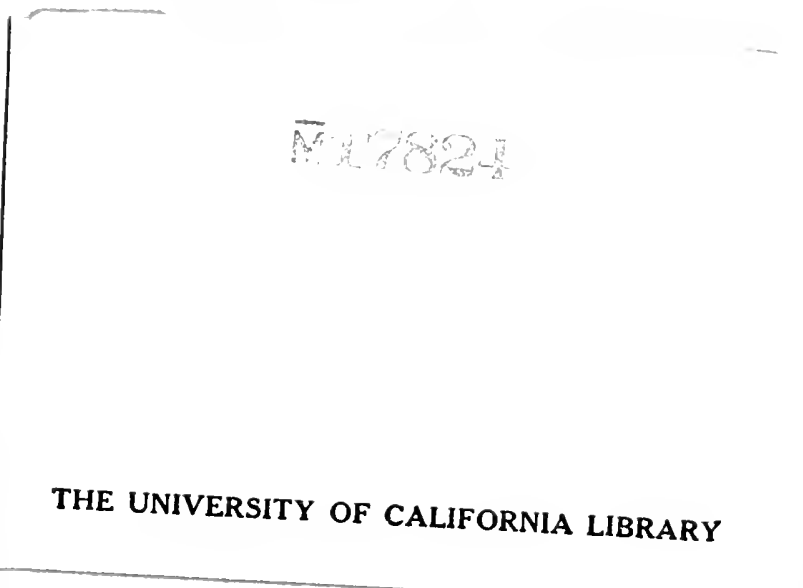




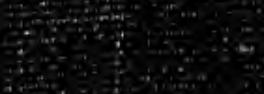

c5.

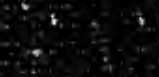

4 